\title{
Groundwater Flow Model for the Reactor Area Final Report
}

by

\author{
M. K. Harris
}

Westinghouse Savannah River Company

Savannah River Site

Aiken, South Carolina 29808

This paper was prepared in connection with work done under the above contract number with the U. S.

Department of Energy. By acceptance of this paper, the publisher and/or recipient acknowledges the U. S. Government's right to retain a nonexclusive, royalty-free license in and to any copyright covering this paper, along with the right to reproduce and to authorize others to reproduce all or part of the copyrighted paper. 


\title{
GROUNDWATER FLOW MODEL FOR THE R-REACTOR AREA
}

\author{
Final Report \\ Prime Contract No. DE-AC09-96SR18500 \\ Subcontract No. AA82275N \\ Prepared for: \\ Westinghouse Savannah River Company \\ Aiken, SC 29808
}

November 13, 1997 


\title{
GROUNDWATER FLOW MODEL FOR THE R-REACTOR AREA Final Report
}

\author{
Prime Contract No. DE-AC09-96SR18500 \\ Subcontract No. AA82275N
}

Prepared for:

Westinghouse Savannah River Company

Aiken, SC 29808

Prepared by:

HydroGeoLogic, Inc.

1155 Herndon Parkway, Suite 900

Herndon, VA 20170

Tel: (703) 478-5186

November 13, 1997 


\section{DISCLAIMER}

This report was prepared as an account of work sponsored by an agency of the United States Government. Neither the United States Government nor any agency thereof, nor any of their employees, makes any warranty, express or implied, or assumes any legal liability or responsibility for the accuracy, completeness, or usefulness of any information, apparatus, product, or process disclosed, or represents that its use would not infringe privately owned rights. Reference herein to any specific commercial product, process, or service by trade name, trademark, manufacturer, or otherwise does not necessarily constitute or imply its endorsement, recommendation, or favoring by the United States Government or any agency thereof. The views and opinions of authors expressed herein do not necessarily state or reflect those of the United States Government or any agency thereof.

This report has been reproduced directly from the best available copy.

Available to DOE and DOE contractors from the Office of Scientific and Technical Information, P.O. Box 62, Oak Ridge, TN 37831; prices available from (615) 576-8401.

Available to the public from the National Technical Information Service, U.S. Department of Commerce; 5285 Port Royal Road, Springfield, VA 22161. 


\section{TABLE OF CONTENTS}

Page

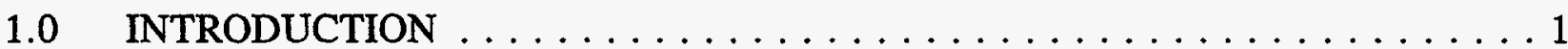

1.1 R-REACTOR SEEPAGE BASINS $\ldots \ldots \ldots \ldots \ldots \ldots \ldots \ldots$

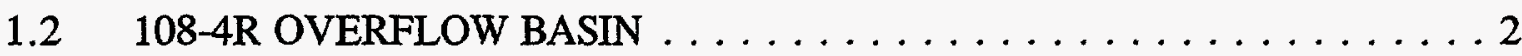

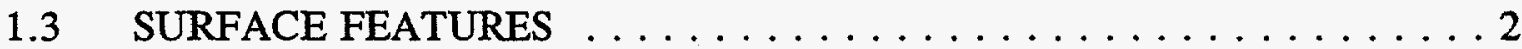

2.0 HYDROGEOLOGIC FRAMEWORK $\ldots \ldots \ldots \ldots \ldots \ldots \ldots \ldots \ldots \ldots \ldots$

2.1 SRS GEOLOGY $\ldots \ldots \ldots \ldots \ldots \ldots \ldots \ldots \ldots \ldots \ldots \ldots \ldots \ldots \ldots$

2.2 HYDROSTRATIGRAPHY OF THE SRS $\ldots \ldots \ldots \ldots \ldots \ldots \ldots$

2.2.1 Meyers Branch Confining System $\ldots \ldots \ldots \ldots \ldots \ldots \ldots$

2.2.2 Floridan Aquifer System $\ldots \ldots \ldots \ldots \ldots \ldots \ldots \ldots \ldots \ldots$

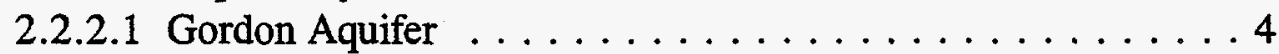

2.2.2.2 Gordon Confining Unit . . . . . . . . . . . 5

2.2.2.3 Upper Three Runs (Water Table) Aquifer . . . . . . . 5

2.3 UNIT-SPECIFIC GEOLOGY AND HYDROGEOLOGY $\ldots \ldots \ldots \ldots 5$

2.3.1 Unit Specific Hydrostratigraphy $\ldots \ldots \ldots \ldots \ldots \ldots \ldots \ldots$

2.3.1.1 "Tan Clay" Interval . . . . . . . . . . . . . . . 6

2.3.1.2 Undifferentiated High Permeability (Transmissive) Zone . . 7

2.3.1.3 "AA Horizon" . . . . . . . . . . . . . . 7

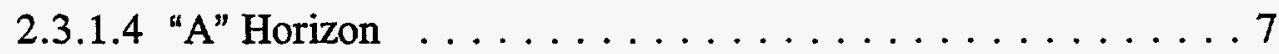

2.3.1.5 Undifferentiated Surface Soils $\ldots \ldots \ldots \ldots \ldots \ldots$

2.4 UNIT-SPECIFIC HYDROGEOLOGY $\ldots \ldots \ldots \ldots \ldots \ldots \ldots$

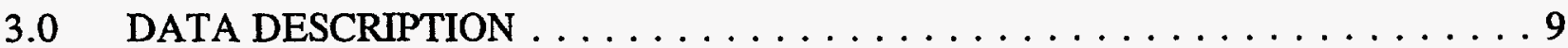

3.1 MONITORING WELL AND BOREHOLE $\ldots \ldots \ldots \ldots \ldots \ldots$

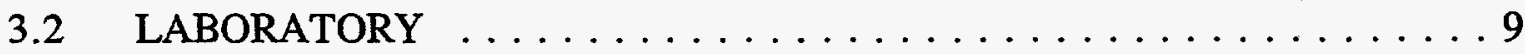

3.3 CONE PENETROMETER $\ldots \ldots \ldots \ldots \ldots \ldots \ldots \ldots \ldots$

3.4 TOPOGRAPHIC SURFACE $\ldots \ldots \ldots \ldots \ldots \ldots \ldots \ldots$

4.0 DELINEATION OF HYDROSTRATIGRAPHIC UNITS $\ldots \ldots \ldots \ldots \ldots \ldots$

$4.1 \quad$ PEN BRANCH FAULT $\ldots \ldots \ldots \ldots \ldots \ldots \ldots \ldots \ldots \ldots \ldots \ldots$

4.2 HYDROSTRATIGRAPHIC SURFACES $\ldots \ldots \ldots \ldots \ldots \ldots$

5.0 GROUNDWATER FLOW MODEL DEVELOPMENT $\ldots \ldots \ldots \ldots \ldots$

5.1 MODEL DISCRETIZATION $\ldots \ldots \ldots \ldots \ldots \ldots \ldots \ldots \ldots \ldots$

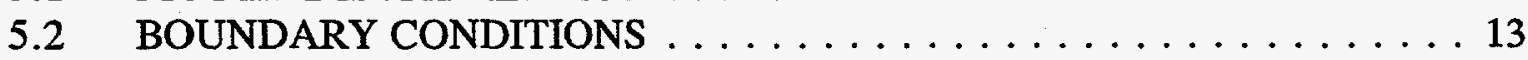

5.3 CONDUCTIVITY FIELD $\ldots \ldots \ldots \ldots \ldots \ldots \ldots \ldots \ldots \ldots$

5.3.1 Mini-Permeability and Laboratory Permeability $\ldots \ldots \ldots \ldots \ldots 16$

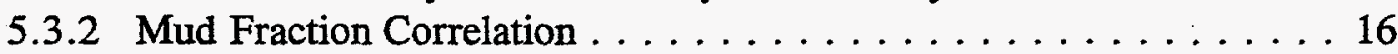

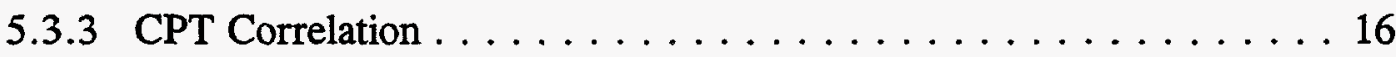

6.0 GROUNDWATER FLOW MODEL RESULTS $\ldots \ldots \ldots \ldots \ldots \ldots \ldots$

6.1 MODEL CALIBRATION $\ldots \ldots \ldots \ldots \ldots \ldots \ldots \ldots \ldots \ldots \ldots \ldots \ldots \ldots$

6.2 HEAD RESIDUALS . . . . . . . . . . . . . . . . 19

6.3 GROUNDWATER RECHARGE/DISCHARGE $\ldots \ldots \ldots \ldots \ldots 19$

6.4 GROUNDWATER FLOW PATTERNS $\ldots \ldots \ldots \ldots \ldots \ldots$ 


\section{TABLE OF CONTENTS}

Page

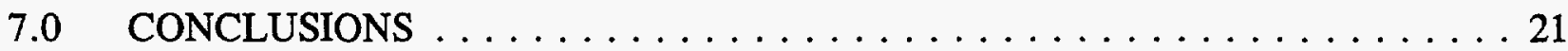

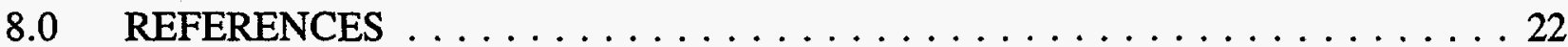

\section{APPENDICES}

Appendix A: Hydraulic Head Data and Residuals

Appendix B: Borehole and CPT Data

Appendix C: Core Mud Fraction Plots

Appendix D: Laboratory Data

Appendix E: Hydrostratigraphic Data

Appendix F: Conductivity Inferred from Core, Laboratory Permeability, and CPT Data Appendix G: Model Calibration Runs 


\section{LIST OF TABLES}

Page

Table 4.1 Offsets South of Pen Branch Fault $\ldots \ldots \ldots \ldots \ldots \ldots \ldots 77$

Table 6.1 Mud Fraction Correlations and Anisotropy Ratios . . . . . . . . . . . . 78

Table 6.2 Friction Ratio Transition Intervals . . . . . . . . . . . . . . 78

Table 6.3 Mini-Permeability Anisotropy Ratios $\ldots \ldots \ldots \ldots \ldots \ldots \ldots \ldots 78$

Table 6.4 Calibration Zones . . . . . . . . . . . . . . . . . . . . . 79

Table 6.5 Summary of Modifications to Initial Conductivity Field . . . . . . . . . . 79

Table 6.6 Calibrated Model Average Conductivities . . . . . . . . . . . . 80

Table 6.7 Average residual and $\mathrm{RMS} \ldots \ldots \ldots \ldots \ldots \ldots \ldots \ldots \ldots$

Table 6.8 Comparison of Measured and Simulated Stream Baseflow . . . . . . . . 81

Table 6.9 Seeds for 3-D Pathlines . . . . . . . . . . . . . . . . . 81 


\section{LIST OF FIGURES}

Page

Figure $1.1 \quad$ R-Reactor Area $\ldots \ldots \ldots \ldots \ldots \ldots \ldots . \ldots \ldots \ldots$

Figure 2.1 Geologic and Hydrogeologic Units for the Savannah River Site. . . . . . . 25

Figure 3.1 Name and Location of Borehole and CPT Data . . . . . . . . . . 26

Figure 3.2 Monitoring Well, Borehole, and CPT Locations . . . . . . . . . . . . . 27

Figure $3.3 \quad 3302$ Maps Obtained from WSRC $\ldots \ldots \ldots \ldots \ldots \ldots \ldots \ldots$

Figure 3.4 Topographic Surface . . . . . . . . . . . . . . . . . . . . . . . . 29

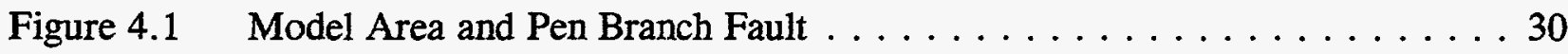

Figure 4.2 Top of Crouch Branch Confining Unit Surface Trend (Prior to faulting) . . 31

Figure 4.3 Top of Gordon Aquifer Surface Trend (Prior to faulting) . . . . . . . . 32

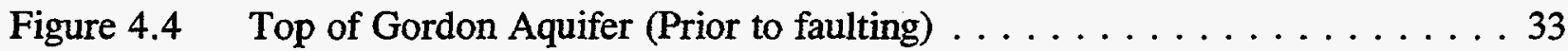

Figure 4.5 Top of Gordon Confining Unit Surface Trend (Prior to faulting) . . . . . 34

Figure 4.6 Top of Gordon Confining Unit (Prior to faulting) . . . . . . . . . . 35

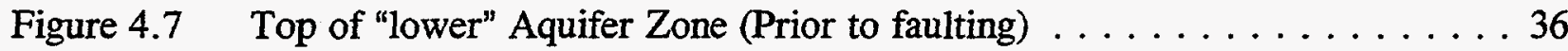

Figure 4.8 Top of "tan clay" Confining Zone (Prior to faulting) $\ldots \ldots \ldots \ldots \ldots$

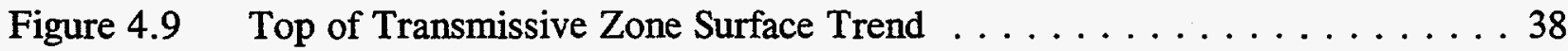

Figure 4.10 Top of Transmissive Zone . . . . . . . . . . . . . . . . . 39

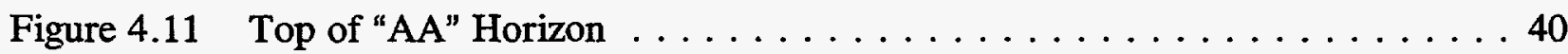

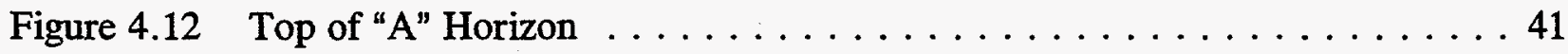

Figure 5.1 Estimated Water Table and Model Boundary . . . . . . . . . . . 42

Figure $5.2 \quad$ R-reactor Area Active mesh Elements . . . . . . . . . . . . . . 43

Figure 5.3 Mesh Cross-Section at $\mathrm{I}=25 \ldots \ldots \ldots \ldots \ldots \ldots \ldots \ldots$

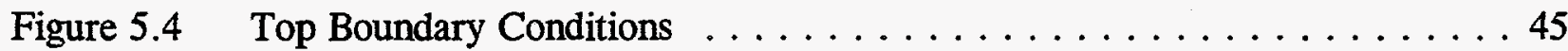

Figure 5.5 Estimated Crouch Branch Aquifer Head . . . . . . . . . . . . 46

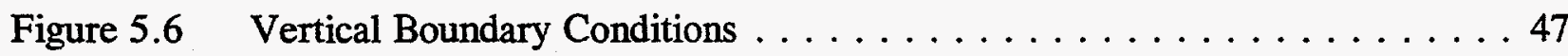

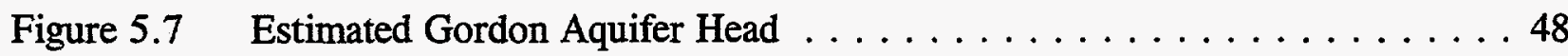

Figure 5.8 Estimated "lower" Aquifer Zone Head . . . . . . . . . . . . . . . . 49

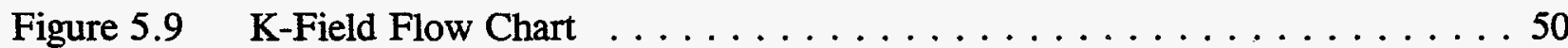

Figure $5.10 \quad K_{\mathrm{h}}$ Distribution for Gordon Aquifer, Element Layer $1 \ldots \ldots \ldots \ldots \ldots$

Figure $5.11 \quad \mathrm{~K}_{v}$ Distribution for Gordon Confining Unit, Element Layer $4 \ldots \ldots \ldots 52$

Figure $5.12 \quad \mathrm{~K}_{\mathrm{h}}$ Distribution for "lower" Aquifer Zone, Element Layer 6 . . . . . . . . 53

Figure $5.13 \quad \mathrm{~K}_{\mathrm{v}}$ Distribution for "tan clay" Confining Zone, Element Layer 7 . . . . . 54

Figure $5.14 \quad \mathrm{~K}_{\mathrm{h}}$ Distribution for Transmissive Zone, Element Layer $10 \ldots \ldots \ldots \ldots 5$

Figure $5.15 \mathrm{~K}_{\mathrm{v}}$ Distribution for "AA" Horizon, Element Layer $13 \ldots \ldots \ldots \ldots \ldots 6$

Figure $5.16 \quad \mathrm{~K}_{\mathrm{v}}$ Distribution for "A" Horizon, Element Layer $16 \ldots \ldots \ldots \ldots$

Figure $5.17 \quad \mathrm{~K}_{\mathrm{v}}$ Distribution for Surface Soil, Element Layer $19 \ldots \ldots \ldots$

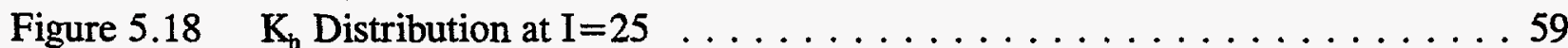

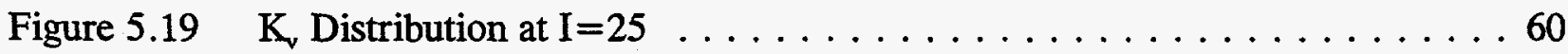




\section{LIST OF FIGURES}

Page

Figure 6.1 Calibration Zones . . . . . . . . . . . . . . . . . . 61

Figure 6.2 Gordon Aquifer and "lower" Aquifer Zone Residuals . . . . . . . . . . . . 62

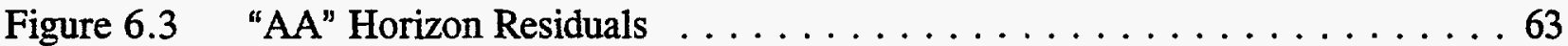

Figure 6.4 "A" Horizon Residuals $\ldots \ldots \ldots \ldots \ldots \ldots \ldots \ldots \ldots \ldots \ldots \ldots$

Figure 6.5 Surface Soil Residuals $\ldots \ldots \ldots \ldots \ldots \ldots \ldots \ldots \ldots \ldots \ldots \ldots$

Figure 6.6 Simulated Groundwater Recharge (discharge) $\ldots \ldots \ldots \ldots \ldots \ldots 66$

Figure 6.7 Discharge Zones . . . . . . . . . . . . . . . . . . . . 67

Figure 6.8 Simulated Head in Gordon Aquifer $\ldots \ldots \ldots \ldots \ldots \ldots \ldots \ldots$

Figure 6.9 Simulated Head in "lower" Aquifer Zone . . . . . . . . . . . . . . . 69

Figure 6.10 Simulated Head in Transmissive Zone $\ldots \ldots \ldots \ldots \ldots \ldots$

Figure 6.11 Simulated Water Table $\ldots \ldots \ldots \ldots \ldots \ldots \ldots \ldots \ldots \ldots \ldots$

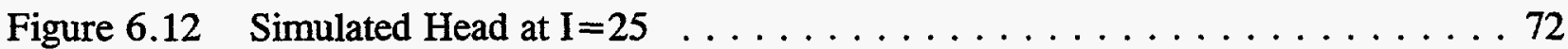

Figure 6.13 Simulated Saturation at $I=25 \ldots \ldots \ldots \ldots \ldots \ldots \ldots \ldots$

Figure 6.14 R-Reactor Area Pathlines with 50 Year Markers . . . . . . . . . . . . . . . 74

Figure 6.15 Pathlines with 50 Year Markers (facing north) $\ldots \ldots \ldots \ldots \ldots \ldots 75$

Figure 6.16 Pathlines with 50 Year Markers (facing west) $\ldots \ldots \ldots \ldots \ldots$ 


\subsection{INTRODUCTION}

A detailed numerical groundwater flow model has been developed for the R-Area of the Savannah River Site in Aiken, South Carolina. The three-dimensional, finite-element groundwater modeling code FACT (Flow and Contaminant Transport) has been used for this study. The code was developed by the Savannah River Technology Center and is based upon numerical algorithms contained in the code SAFT3D, which was developed by HydroGeoLogic, Inc. The necessary QA/QC has been completed on this code (WSRC, 1995). The model incorporates lithological descriptions, cone penetrometer data, mini-permeameter data, and hydraulic head data. The model encompasses an area that surrounds the R-Reactor Area. The area of interest for this model is the R-Reactor Seepage Basins. However the model can be used for other waste units within the RReactor Area.

One objective of this study is to develop a detailed flow model to guide future characterization efforts. In the past, groundwater flow models have been developed after the characterization of a waste unit is complete. Only the initial characterization for the R-Reactor Seepage Basins has been completed. This model study will identify data gaps and lead to recommendations for further characterization that will be completed as part of the Phase II characterization of the area.

A second objective of the model development is to provide a foundation for future contaminant transport simulations. Transport modeling of the contaminants of concern will be addressed under the next phase of groundwater modeling. This phase will incorporate the new characterization data.

\section{$1.1 \quad$ R-REACTOR SEEPAGE BASINS}

The R-Reactor Seepage Basins are six unlined basins (excavated 6.6 feet to 16.4 feet below land surface [ft bls]) located in the abandoned reactor construction staging area north of the R-Reactor building (105-R). The basins were constructed and operated between 1957 and 1964 and received low-level radioactive wastewater (an estimated 4,808,440 gallons), via process sewer line, from purges of the R-Reactor disassembly basin (Pekkala et al., 1987). These purges were necessary to keep the tritium concentration in the disassembly basin water below the level that ensures safe working conditions. The primary radionuclides present in the purge water were tritium (as HTO), Strontium-90 $\left.{ }^{90} \mathrm{Sr}\right)$ and Cesium-137 $\left({ }^{137} \mathrm{Cs}\right)$.

R-Reactor began operation in 1953. In 1957, a calorimeter experiment failure occurred, and purge water containing high-level radioactive contamination were released to the emergency disassembly basin. Subsequent purging of water from the disassembly basin resulted in approximately 2700 Curies (Ci) of activity being released primarily to basin 1 , and to a lesser extent, basins $2-4$ (basins $2-6$ were placed in operation after the incident). It is estimated that the $2700 \mathrm{Ci}$ was composed of $200 \mathrm{Ci}$ of ${ }^{90} \mathrm{Sr}, 1000 \mathrm{Ci}$ of ${ }^{137} \mathrm{Cs}$, and $1500 \mathrm{Ci}$ of other non-volatile beta emitters (primarily radionuclides with half-lives less than 3 years).

Basin 1 was deactivated and backfilled in January 1958 because of surface outcrop and leakage to an abandoned construction sewer line system left from the former use of the area as a reactor construction staging area. In 1960, basins 2-5 were deactivated and backfilled. The ground surface above basins 1-5 was treated with herbicide and covered with a thin layer of asphalt emulsion. In addition, a kaolinite dike (down to the clay layer) was constructed around basin 1 and the northwest end of basin 3 to contain lateral movement of radionuclides. Clay caps were placed 
over the diked areas to control exposure to radioactivity contaminated soils and infiltration of surface water. Basin 6 was last used in 1964 and backfilled in 1977. All basins were backfilled with kaolin.

Environmental monitoring, including limited soil sampling, extensive groundwater monitoring, and vegetation sampling surveys, have been conducted since 1957. Based on numerous sampling surveys, it has been concluded that radionuclides have migrated out of the seepage basins via percolation and through the abandoned construction sewer line. Studies indicate that this line has been breached and acted as a conduit for the migration of radionuclides.

Radionuclide activities in basin soils have been encountered up to 8,000 nanoCuries per gram (nCi/g) ${ }^{137} \mathrm{Cs}$ and $41 \mathrm{nCi} / \mathrm{g}{ }^{90} \mathrm{Sr}$. Vegetation activities up to $32 \mathrm{nCi} / \mathrm{g}{ }^{137} \mathrm{Cs}$ and $16 \mathrm{nCi} / \mathrm{g}{ }^{90} \mathrm{Sr}$ have been detected. Tritium, gross alpha, non-volatile beta, cadmium, and lead have been detected in the groundwater above drinking water standards.

The R-Reactor Seepage Basins were covered in 1996 with asphalt to minimize plant and animal intrusion. The asphalt cover also substantially reduces percolation of rain water. However, to simulate initial conditions the asphalt cover was not included in the groundwater flow model for this phase.

\section{$1.2 \quad 108-4 R$ OVERFLOW BASIN}

The 108-4R Overflow Basin is located 250 feet southwest of building 105-R and approximately 500 feet southwest of R-Reactor Seepage Basins. This basin was constructed to collect incidental overflow from two adjacent underground storage tanks. It was in operation from 1953 to 1964. Original dimensions of the unlined basin were approximately 60 feet $x 60$ feet $\times 8$ feet deep. A soil berm up to 2 feet above grade was placed around the basin's perimeter.

The basin was removed from service in 1964 and is presently inactive. It is partially filled with an estimated 5 feet of sediment. Soil sampling results from the basin indicate that the soils contain elevated concentrations of petroleum hydrocarbons (maximum total petroleum hydrocarbons [TPH] value 4,750 ppm) and benzene, toluene, ethylbenzene, and xylene (BTEX) compounds (up to $40 \mathrm{ppm})$.

\subsection{SURFACE FEATURES}

R Area is located in the east-central part of SRS approximately $8.3 \mathrm{~km}$ (5.2 miles) from the nearest SRS boundary and $2.7 \mathrm{~km}$ (1.7 miles) northeast of Par Pond. The RRSBs lie north of and adjacent to $\mathrm{R}$ Reactor on an elevated divide between Mill Creek and the primary effluent canal northeast of the R-Area perimeter fence (see Figure 1.1). Topographic relief in this area is low, generally

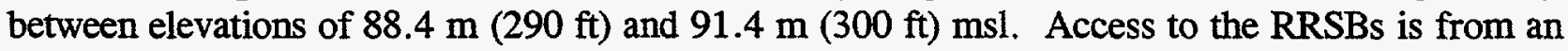
unpaved road just off Road G-1. The Overflow Basin is located southwest of the 105-R building and approximately $152.4 \mathrm{~m}(500 \mathrm{ft})$ southwest of the RRSBs, and can be accessed inside the 105-R perimeter fence.

Figure 1.1 shows the drainage basins and drainage patterns, resulting from the gentle topography in the area, as well as surface water bodies potentially affected by the Unit. There are no surface water bodies on the Unit itself nor are Carolina bays present in the vicinity of R Reactor. Surface runoff from the RRSBs flows either north and west into Mill Creek or east into the R-Effluent 
Canal. Mill Creek is part of the Tinker Creek Basin and the R-Effluent Canal is part of Par Pond Basin. The Par Pond Basin receives surface runoff flowing east, south and southwest from the RReactor area. 


\subsection{HYDROGEOLOGIC FRAMEWORK}

\subsection{SRS GEOLOGY}

The SRS is underlain by sediment of the Atlantic Coastal Plain. The Atlantic Coastal Plain consists of a southeast-dipping wedge of unconsolidated and semi-consolidated sediment that extends from its contact with the Piedmont province at the Fall Line to the edge of the continental shelf. The sediment ranges from Late Cretaceous to Miocene in age and comprises layers of sand, muddy sand, and mud with subordinate calcareous sediment. The sediment rests uncomfortably on crystalline and sedimentary basement rock.

Only the hydrostratigraphy will be discussed in this report. Detailed descriptions of the geology of the SRS can be found in several recent reports (Colquhoun and others, 1983; Fallaw and others, 1990; Aadland and others, 1991; Dennehy and others, 1989; Logan and Euler, 1989; Fallaw and Price, 1995).

\subsection{HYDROSTRATIGRAPHY OF THE SRS}

The hydrostratigraphy of the SRS has been subject to several different classifications. This report incorporates the hydrostratigraphic nomenclature currently established for the SRS vicinity by Aadland and others (1995). The nomenclature is correlated with the local lithostratigraphy in Figure 2.1. A thorough description and review of the hydrostratigraphy of the SRS region are available in Aadland and others (1995). This report address the up-dip part of the Floridan aquifer system and the top of the Meyers Branch Confining System as defined by Aadland and others (1995)(Figure 2.1).

\subsubsection{Meyers Branch Confining System}

The Meyers Branch Confining System defines the base of the Floridan aquifer system in the study area. The top of the Meyers Branch Confining System is delineated by the laterally continuous, dense, gray to black, clay and sandy clay of the Lang Syne Formation of the Black Mingo Group (Figure 2.1) (Aadland and others, 1991, 1995).

\subsubsection{Floridan Aquifer System}

\subsubsection{Gordon Aquifer}

The lowermost unit that is characterized in this report is the Gordon aquifer (Figure 2.1). The Gordon aquifer constitutes the basal unit of the Floridan aquifer system in this part of the SRS Region. The Gordon aquifer is made up of the loose sand and clayey sand of the Congaree Formation and, where present, the sandy parts of the underlying Fishburne and Williamsburg Formations (Figure 2.1) (Harris and others, 1990; Aadland and others, 1991, 1995). The sand within the Gordon aquifer is yellowish to grayish orange in color and is sub- to well-rounded, moderately to poorly sorted, and medium to coarse-grained. Pebbly layers and zones of iron and silica cement are common. Layers of light tan to gray clay up to three feet in thickness are rare, and thin (less than 6 inches in thickness) layers of clay are often found near the base of this unit. Small amounts of calcareous sediment have been found sporadically within the Gordon aquifer in the study area. 


\subsubsection{Gordon Confining Unit}

The Gordon aquifer is separated from the Water Table aquifer by the Gordon confining unit. The unit is commonly referred to as the "green clay" in previous SRS literature and includes sediment of the Warley Hill Formation (Figure 2.1). The unit comprises layers of interbedded silty and clayey sand, sandy clay and clay. The clay is stiff to hard and is often fissile. Glauconite is a common constituent and imparts a distinctive greenish cast to the sediment, hence the informal name "green clay" for this unit. The Gordon confining unit includes some calcareous sediment and limestone, primarily calcarenaceous sand and clayey sand with subordinate calcarenaceous and micritic clay, and sandy micrite and limestone.

\subsubsection{Upper Three Runs (Water Table) Aquifer}

The Water Table aquifer is equivalent to the Upper Three Runs aquifer of Aadland and others (1995). The Water Table aquifer includes all sediment from the ground surface to the top of the Gordon confining unit. The Water Table aquifer incorporates the "upland" unit, the Tobacco Road Sand, Dry Branch Formation, Clinchfield Formation, and Santee Limestone (Figure 2.1). The Water Table aquifer is locally divided into informal "lower" and "upper" aquifer zones separated by the "tan clay" confining zone.

"Lower" Upper Three Runs (LUTR) Aquifer Zone. The "lower" aquifer zone consists of the dominantly fine-grained, well-sorted sand and clayey sand of the Santee Limestone and parts of the Dry Branch Formation beneath the "tan clay" confining zone (Figure 2.1). Descriptions of drill core indicate that the carbonate sediment in this vicinity has a dominant siliciclastic component, and consists of calcarenaceous sand, micritic sand, shelly sand and some sandy calcarenite and shelly limestone.

"Tan Clay" Confining Zone. The "tan clay" confining zone is equivalent to the "tan clay" zone referred to in previous SRS reports. The "tan clay" confining zone includes sediment of the Dry Branch Formation (Figure 2.1). The zone contains light-yellowish tan to orange clay and sandy clay interbedded with clayey sand and sand. The zone contains light-yellowish tan to orange clay and sandy clay interbedded with clayey sand and sand. The lithology of the clay is similar to that of the Twiggs Clay Member, but is dispersed vertically and horizontally and is not continuous over long distances (Harris and others, 1990; Aadland and others, 1991).

"Upper" Upper Three Runs (UUTR) Aquifer Zone. The "upper" aquifer zone consists of all sediment from the ground surface to the top of the "tan clay" confining zone. The "upper" aquifer zone includes the "upland" unit, Tobacco Road Sand, and part of the Dry Branch Formation (Figure 2.1). This unit is characterized by sand and clayey sand with minor intercalated clay layers. The sediment within the "upland" unit is commonly very dense and clayey and contains some gravelly sand.

\subsection{UNIT-SPECIFIC GEOLOGY AND HYDROGEOLOGY}

A field investigation completed in 1995 and 1996 provided data that allowed the correlation of local and regional geology/hydrology of the Tertiary Aquifer System in the vicinity of the RRSB. Specific field information collected during that investigation included core data, lithologic and stratigraphic data, water level data, geophysical logs, cone penetrometer data, and permeability data. These correlation's and composite lithology/CPT logs were used to develop cross sections 
of the shallow strata as well as structure contour and isopach maps of select stratigraphic units. The lithologic data were then used to define the stratigraphy of the Tertiary aquifers for incorporation into the groundwater flow and transport model.

\subsubsection{Unit Specific Hydrostratigraphy}

Based on the lithologic data collected during the recent investigation, the hydrostratigraphy above the "Tan Clay" has been subdivided into several distinct horizons that are correlatable in the vicinity of the RRSB. These horizons consist of clay and clayey sand which may affect groundwater infiltration and flow within the uppermost saturated zone. These horizons are termed, from deepest to shallowest shallowest to deepest: - the "Tan Clay" interval, an undefined high permeability zone, the "AA" horizon; the " $\mathrm{A}$ " horizon; and the undifferentiated surface soil (Figure 2.1). Appendix E contains elevations and thickness' for the " $A$ " and " $A A$ " horizons, the undefined high permeability zone and the "Tan Clay" interval. Cross-sections, structure and isopach maps are available in WSRC (1997b) The following summaries describe the occurrence and nature of these horizons in the vicinity of the RRSB. All of these data were incorporated to the groundwater flow model.

\subsubsection{1 "Tan Clay" Interval}

In the study area the "Tan Clay" represents a semi-confining zone. The clay occurs as lenses and is interbedded with sands, silty sands, clayey sands, and sandy clays. For hydrostratigraphic purposes in this report the "Tan Clay" zone is referred to as an interval.

The upper surface of the "Tan Clay" interval occurs at a depth of 30.5 to $38 \mathrm{~m}$ (100 to $125 \mathrm{ft}) \mathrm{bls}$, and ranges in elevation between 61.7 and $85.1 \mathrm{~m}(202.3$ and $279.3 \mathrm{ft}) \mathrm{msl}$. The "Tan Clay" interval occurs in a relatively uniform thickness across the site ranging from 7.6 to $8.2 \mathrm{~m} \mathrm{(20}$ to $27 \mathrm{ft})$. The upper contact of the unit is at $61.8 \mathrm{~m}(202.6 \mathrm{ft}) \mathrm{msl}$ in the northwestern portion of the site and decreases in elevation toward the east and south. The lowest elevation of the upper contact of the "Tan Clay" interval is at $52.5 \mathrm{~m}(175.7 \mathrm{ft}) \mathrm{msl}$ at RCH-1 to $47 \mathrm{~m}(154.3 \mathrm{ft}) \mathrm{msl}$.

The upper and lower boundaries of the "Tan Clay" interval were selected from lithology and CPT analyses. The contact can be distinguished by "the relatively low tip stress as compared to the overlying " $\mathrm{AA}$ " horizon. This characteristic signature indicates a finer grained matrix than in the overlying "AA" horizon. Although the "Tan Clay" includes variable lithology, the unit contacts also correspond to a very high positive deflection in measured pore pressures. The measured pore pressures are fairly low in all uphole sections measured, and the positive deflection within the "Tan Clay" is generally the highest in the CPT holes. In addition, the upper contact of the "Tan Clay" interval corresponds to a positive deflection in the gamma counts.

The materials of the "Tan Clay" interval are described as sand, clayey sand, sandy clay and clay that are poorly to very poorly sorted, very fine grained to pebble sized, and subangular to subrounded. Although clay and sand content vary within the unit, the average sand content for the "Tan Clay" interval in the study area is $79.1 \%$, and the average fines content is approximately $13 \%$. Gravel is common within the horizon, and ranges from $0 \%$ to $15 \%$ within individual samples. Secondary diagnostic features include clay balls, which are commonly found near the lower contact of the horizon, and common black manganese oxide staining and laminae. 


\subsubsection{Undifferentiated High Permeability (Transmissive) Zone}

Underlying the "AA" horizon is a sandy, high-permeability interval.Strata within this interval are predominantly sand to silty sands with interbedded clay and pebble layers. Thickness at the interval ranges from approximately $15.2 \mathrm{~m}(50 \mathrm{ft})$ in the western portion of the study area to approximately $7.6 \mathrm{~m}(25 \mathrm{ft})$ in the eastern portion of the study area.

\subsubsection{3 "AA Horizon"}

Underlying the " $\mathrm{A}$ " horizon is a more sandy interval that has been termed the "AA" horizon. The upper surface of the "AA" horizon occurs at a depth of 9.1 to $21.3 \mathrm{~m}$ (30 to $70 \mathrm{ft}$ ) across the site, and ranges in elevation from 69.5 to $82.9 \mathrm{~m}(228$ to $272 \mathrm{ft}) \mathrm{msl}$. Thickness of the horizon ranges from 7 to $8.8 \mathrm{~m} \mathrm{(23} \mathrm{to} 30.5 \mathrm{ft}$ ), and averages $7.7 \mathrm{~m}(25.6 \mathrm{ft})$.

The upper boundary of the "AA" horizon was selected from CPT analyses because this contact can be distinguished by the relatively high tip resistance as compared to the overlying " $A$ " horizon. These characteristics represents signatures which signify a coarser grained matrix than in the overlying " $\mathrm{A}$ " horizon. In addition, the gamma signature within the "AA" horizon is highly variable, indicating interbedded and interlayered sands, silts and clays.

The "AA" horizon is described as clayey sand and sand that is poorly to very poorly sorted, very fine grained to pebble sized, and subangular to subrounded. The color is light red, medium red and light purple at the upper contact of the horizon in coreholes.

\subsubsection{4 "A" Horizon}

The "A" horizon is the shallowest of the identified hydrostratigraphic units, and appears to act as a shallow semi-confining unit. Several small areas of perched water have historically occurred at the RRSB (above the " $\mathrm{A}$ " horizon), and are depicted on historical potentiometric maps. The upper surface of the clayey zone which is designated as " $\mathrm{A}$ " horizon, occurs at a depth of 1 to 10 $\mathrm{m}$ ( 3 to $33 \mathrm{ft}$ ) bls, and ranges in elevation from 79.7 to $81.3 \mathrm{~m}(261.5 \mathrm{ft}$ to $299.6 \mathrm{ft}) \mathrm{msl}$. Thickness of the unit ranges from $5.8 \mathrm{~m}$ to $14.3 \mathrm{~m}$ (19 to $47 \mathrm{ft}$ ), and averages $9.2 \mathrm{~m}(30.2 \mathrm{ft})$.

The "A" horizon increases in thickness toward the east central portion of the RRSB, and dips to the southeast in the study area. The boundaries of the " $A$ " horizon were selected based on lithology and CPT analyses. The "A" horizon has a characteristic signature of low tip pressure throughout the unit, and a high friction ratio. These characteristic signatures are typical of a finegrained matrix with some clay. Although the horizon includes sands, very coarse-grained material is almost nonexistent in the section. Sleeve pressures throughout the CPT holes were relatively consistent within the " $A$ " horizon.

The " $\mathrm{A}$ " horizon is described as a clayey sand, sandy clay and sand. The color varies, but is described as reddish brown, reddish yellow and brownish yellow near the upper contact of the unit, and grades to gray, grayish purple, and medium purple with depth. Clay balls are common, especially at the middle and basal portions of the unit. The " $\mathrm{A}$ " horizon's fine-grained nature and relatively low vertical and horizontal permeabilities will impede downward movement of groundwater compared to the underlying " $\mathrm{AA}$ " horizon and deeper coarse-grained zones. Groundwater occurs under water table conditions above and within " $\mathrm{A}$ " horizon. The low porosity of the upper portion of the unit likely results in the perched water which occurs in several portions 
of the northern section of the study area, creating a apparent radial pattern of groundwater movement above and within the upper portion of the "A" horizon in several of the historical potentiometric maps.

\subsubsection{Undifferentiated Surface Soils}

The shallow surface soil in the vicinity of the Unit consists of interbedded sands, silts, and clays that overlie the "A" horizon. These strata range in thickness from approximately 1.0 to $10 \mathrm{~m}$ (3 to $33 \mathrm{ft}$ ) and generally thicken from northwest to southeast across the RRSB. These strata are correlatable to the Upland Unit that is of fluvial origin (Aadland et al., 1995).

\subsection{UNIT-SPECIFIC HYDROGEOLOGY}

The water table aquifer extends to the top of the Gordon Confining Unit as the Upper Three Runs (UTR) Aquifer (Figure 2.1). The water table is divided into upper and lower sections to correspond with the saturated zone above and below the "Tan Clay", respectively. The saturated zone from the water table to the top of the "Tan Clay" interval are identified as the UUTR Aquifer, and the zone between the "Tan Clay" and the Gordon Confining Unit are discussed separately as the LUTR Aquifer.

The UUTR Aquifer is between 24.4 and $30.5 \mathrm{~m}$ (80 and $100 \mathrm{ft}$ ) thick in the seepage basin area, and includes the " $\mathrm{A}$ " horizon, the "AA" horizon, and the undifferentiated materials above the "Tan Clay". As previously discussed, the " $A$ " horizon is semi-confining, and supports perched water zones in several portions of the study area. A majority of the historical water level monitoring data and data collected for this investigation are from the UUTR Aquifer, and typically from wells screened in the " $A$ " horizon. Water level data from the lower section of the UUTR Aquifer is limited, and the hydrology of these portions of the aquifer in the vicinity of the Unit are poorly understood.

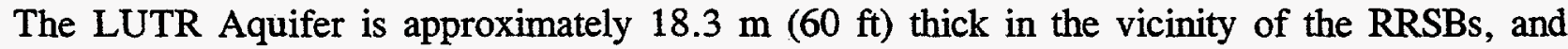
includes interbedded clays, sands, and carbonates. Branch Formation and the Tinker/Santee Formation. Historical and recent water level monitoring data in this portion of the UTR Aquifer is sparse, and therefore the interpretation of the hydrology of these portions of the aquifer in the vicinity of the RRSB is limited.

The "green clay" underlies the LUTR aquifer. There is limited information on the "green clay" in R-Area. The Gordon aquifer underlies the "green clay". The Gordon aquifer is of moderately high hydraulic conductivity and is regional in extent south of Upper Three Runs Creek. Groundwater flow in the Gordon Aquifer is towards Upper Three Runs Creek and the Savannah River, depending upon the location at the SRS.

The Crouch Branch Confining Unit, which represents the top of the Meyers Branch Confining System underlies the Gordon Aquifer. This confining unit is composed of low permeability materials. The Crouch Branch Confining unit is the base of the groundwater flow model. 


\subsection{DATA DESCRIPTION}

\subsection{MONITORING WELL AND BOREHOLE}

Monitoring well water levels have been monitored on a quarterly basis in the R-Reactor Area. Quarterly monitoring well data from the first quarter of 1986 to the second quarter of 1996 were obtained from WSRC and used to compute an average water level for each monitoring well. The average water levels were calibration targets for the groundwater flow model. Appendix A contains a list of the 55 average water levels at the R-Reactor Area. Monitoring wells RBP-1D, RBP-2D, and RBP-3D were constructed and measured in the second quarter of 1997.

Hydrologic data were obtained from continuous drill cores at 8 boreholes in the R-Reactor Area. Appendix B lists the borehole data. The foot-by-foot description of mud fraction (silt and clay sized material) was used to produce the conductivity field. Appendix $\mathrm{C}$ contains plots of mud fraction (smoothed) versus elevation ( $\mathrm{ft}$ ) corresponding to the 8 cores. The dashed lines represent the hydrostratigraphic picks from WSRC (1997b). The raw mud fraction data were smoothed using the following filter which rejects frequencies in the upper half of the spectrum (Hamming, 1973):

$$
h_{k}=\frac{f_{k-3}+2 f_{k-2}+3 f_{k-1}+3 f_{k}+2 f_{k+1}+f_{k+2}}{12}
$$

where $f_{k}$ is the raw mud fraction data and $h_{k}$ is the smoothed mud fraction. The no recovery zones found in the raw mud fraction data are assumed to be sandy soils with an average mud fraction of $3 \%$. The no recovery zones were replaced with a $3 \%$ mud fraction.

\subsection{LABORATORY}

Laboratory permeability data from 22 soil samples of the R-Reactor Area are presented in WSRC (1997b). Samples were taken at RCH-1, RPC-1, RPC-2, and RPT-6. Laboratory permeability data at P-20 are presented in Bledsoe (1987). Celeste (1997) performed mini-permeability tests on the following cores: RPC-2CL, RPC-1CL, RCH-1, RBW-2CL, and RBW-2CU. Appendix D presents all the laboratory data.

\subsection{CONE PENETROMETER}

Cone penetrometer tests (CPT) were conducted adjacent to the R-Reactor seepage basins to collect lithologic information (WSRC, 1997b). Appendix B lists the CPT locations. As the penetrometer probe is forced into the ground, the conical tip and friction sleeve independently measure vertical resistance beneath the tip as well as frictional resistance on the side of the probe as functions of depth. The penetrometer probe also has a pressure transducer in the cone that measure the pore water pressure and a resistivity module that measure variance in soil conductance. The friction ratio, pore pressure, sleeve stress, and tip resistance data were used to produce the conductivity field.

The borehole data and CPT data were analyzed by WSRC (1997b) to determine the stratigraphic layering presented in Appendix E. Figure 3.1 shows the name and location of the borehole and 
CPT data. Figure 3.2 shows the location of the monitoring well data, borehole data, and CPT data along with a basemap of the modeled area.

\subsection{TOPOGRAPHIC SURFACE}

The topographic surface is constructed using USGS Digital Elevation Model (DEM) data and 3302 survey maps. The 3302 maps were digitized near streams and man-made structures and used to supplement the DEM data. The 3302 maps obtained from WSRC are shown in Figure 3.3 and the topographic surface for the R-Reactor Area is shown in Figure 3.4. 


\subsection{DELINEATION OF HYDROSTRATIGRAPHIC UNITS}

The construction of the boundaries of hydrostratigraphic units incorporated regional scale hydrogeologic framework data from (Aadland, et al., 1995) and site scale Phase I investigation data (WSRC, 1997b). The regional scale hydrogeologic framework data includes the following:

- Top of Crouch Branch Confining Unit

- Top of Gordon Aquifer

- Top of Gordon Confining Unit

- $\quad$ Pen Branch fault

The Phase I investigation data includes the following:

- Top of Gordon Aquifer

- Top of Gordon Confining Unit

- Top of "lower" aquifer zone

- Top of "tan clay" confining zone

- Top of transmissive zone

- Top of "AA" horizon

- Top of "A" horizon

\subsection{PEN BRANCH FAULT}

The Pen Branch fault is located south of the R-Reactor Area and extends east and west of the RReactor Area. For convenience, the model area is aligned with the Pen Branch fault, which is a prominent structural feature. The fault is approximated by a straight line in an areal view. Vertically, the fault is modeled as a continuous linear shift of the hydrostratigraphic units over a short interval. The fault offset is assumed constant at a given elevation. Figure 4.1 shows the model area and the Pen Branch fault. Offsets south of Pen Branch fault were estimated from Aadland, et al., 1995 and are given in Table 4.1.

\subsection{HYDROSTRATIGRAPHIC SURFACES}

The hydrostratigraphic surfaces were produced using the following two-step procedure:

- $\quad$ Surface Trend Step:

- $\quad$ Perform 2-D kriging of all hydrostratigraphic data on a uniform grid.

- $\quad$ Constrain Step:

- $\quad$ Remove surface trend grid points within $2000 \mathrm{ft}$ of Phase I hydrostratigraphic "picks."

- Use linear triangular functions to interpolate the Phase I hydrostratigraphic "picks" and the remaining surface trend grid points.

The top of the Crouch Branch Confining Unit surface trend is shown in Figure 4.2. The shaded circles represent digitized contours from Aadland et al. (1995, Plate 16). The contour data south of Pen Branch fault was offset $-40 \mathrm{ft}$ so that a continuous surface will result from the surface trend step. The constrain step was not performed since Phase I hydrostratigraphic data is absent. The 
final top of the Crouch Branch Confining Unit is obtained by offsetting the continuous surface by $+40 \mathrm{ft}$ south of Pen Branch fault to restore the fault.

The top of the Gordon Aquifer surface trend is shown in Figure 4.3. The shaded circles represent digitized contours (selected points on the contours) from Aadland et al. (1995, Plate 14). The shaded circle P's represent Phase I hydrostratigraphic data. The contour data south of Pen Branch fault was offset $-40 \mathrm{ft}$ so that a continuous surface will result from the surface trend step. Figure 4.4 shows the top of Gordon Aquifer after the constrain step. The final top of Gordon Aquifer is obtained by offsetting the continuous surface by $+40 \mathrm{ft}$ south of Pen Branch fault to restore the fault.

The top of Gordon Confining Unit surface trend is shown in Figure 4.5. The shaded circles represent digitized contours from Aadland et al. (1995, Plate 12). The shaded circle P's represent Phase I hydrostratigraphic data. The contour data south of Pen Branch fault was offset $-40 \mathrm{ft}$ so that a continuous surface will result from the surface trend step. Figure 4.6 shows the top of Gordon Confining Unit after the constrain step. The final top of Gordon Confining Unit is obtained by offsetting the continuous surface by $+40 \mathrm{ft}$ south of Pen Branch fault to restore the fault.

Figure 4.7 shows the top of "lower" aquifer zone after the two-step procedure with the shaded circles representing Phase I hydrostratigraphic data. The final top of "lower" aquifer zone is obtained by offsetting the continuous surface by $+20 \mathrm{ft}$ south of Pen Branch fault to restore the fault.

Figure 4.8 shows the top of "tan clay" confining zone after the two-step procedure with the shaded circles representing Phase I hydrostratigraphic data. The final top of "tan clay" confining zone is obtained by offsetting the continuous surface by $+20 \mathrm{ft}$ south of Pen Branch fault to restore the fault.

The top of transmissive zone surface trend is shown in Figure 4.9. The shaded circles represent Phase I hydrostratigraphic data and the digitized Phase I contours (WSRC, 1997b, Figure 3-7). Figure 4.10 shows the top of transmissive zone after the two-step procedure with the shaded circles representing Phase I hydrostratigraphic data.

Phase I hydrostratigraphic data and digitized Phase I contours (WSRC, 1997b, Figure 3-5) are used to construct th top of "AA" horizon surface trend. Figure 4.11 shows the top of "AA" horizon after the two-step procedure with the shaded circles representing Phase I hydrostratigraphic data.

Phase I hydrostratigraphic data and digitized Phase I contours (WSRC, 1997b, Figure 3-4) are used to construct the top of ' $\mathrm{A}$ ' horizon surface trend. Figure 4.12 shows the top of " $\mathrm{A}$ " horizon after the two-step procedure with the shaded circles representing Phase I hydrostratigraphic data. 


\subsection{GROUNDWATER FLOW MODEL DEVELOPMENT}

Development of the R-Reactor area groundwater flow model followed the development of the General Separation Area groundwater flow model (WSRC, 1997a).

\subsection{MODEL DISCRETIZATION}

Groundwater recharge over the R-Reactor Area travels as deep as the sandy transmissive zone before discharging to Mill Creek, Pond A, Pond C, Pond 5 or tributary. The vertical boundaries are generally defined along natural no-flow boundary conditions along streams, ponds and canals. Exception is the southern boundary where prescribed head is applied between Pond 2 and Pond 5 and between Pond 5 and Pond C. The western no-flow boundary is defined using the regional water table map obtained from Hiergesell (1997). Figure 5.1 shows the estimated water table and the model boundary.

The model discretization is approximately aligned with the Pen Branch fault and the areal resolution is $200 \mathrm{ft}$ by $200 \mathrm{ft}$ surrounding the R-Reactor Seepage Basins. There are 64 elements along the east-west axis and 61 elements along the north-south axis. The top of the threedimensional mesh conforms to the ground surface and the bottom of the mesh conforms to the top of the Crouch Branch Confining Unit. Interior nodal layers conform to the top of the Gordon Aquifer, top of the Gordon Confining Unit, top of the "lower" aquifer zone, top of the "tan clay" confining zone, top of the transmissive zone, top of the "AA" horizon, and top of the " $A$ " horizon. The transmissive zone, "AA" horizon, "A" horizon, and surface soils are each represented by three finite-elements in the vertical direction. The Gordon Aquifer, Gordon Confining Unit, "lower" aquifer zone, and "tan clay" confining zone are each represented by two finite-elements in the vertical direction. The mesh size is $64 \times 61 \times 20=78080$ elements or $65 \times 62 \times 21=84630$ nodes. Both areally and vertically, the mesh is finer near the R-Reactor Seepage Basins. Figure 5.2 shows an areal view of the active elements and the shaded area highlights the two elemental rows representing the Pen Branch fault. The Pen Branch fault is modeled as a linear shift over a two element wide fault interval. Figure 5.3 shows a cross-section of the mesh at the $25^{\text {th }}$ north-south nodal plane.

\subsection{BOUNDARY CONDITIONS}

The top boundary conditions are illustrated in Figure 5.4. The ponds and canals are modeled with a general source head boundary condition with leakance coefficient of $1.0 \mathrm{~d}^{-1}$ and source head set to the current water level. The remaining area is assigned a combination recharge/drain boundary condition or no-flow boundary condition. The no-flow boundary condition represents buildings 105-R and 186-R. The remaining area is assigned a combination recharge/drain boundary condition with a maximum recharge rate of $18 \mathrm{in} / \mathrm{yr}$ and drain coefficient of $1.0 \mathrm{~d}^{-1}$, which is the same as the recharge/drain boundary condition used in the General Separation Area flow model (WSRC, 1997a).

The bottom of the model is assigned a general source head boundary condition with leakance coefficient of $3 \times 10^{-6} \mathrm{~d}^{-1}$ and source head from the Crouch Branch Aquifer. Two-dimensional kriging of digitized head contours from Aadland et al.(1995, Plate 45) produces the estimated Crouch Branch Aquifer head shown in Figure 5.5. The shaded circles represent the digitized head contours. 
The vertical boundaries are assigned either a no-flow or prescribed head boundary condition. The eastern, northern, and western boundary nodes are assigned prescribed head for the Gordon Aquifer and "lower" aquifer zone with no-flow elsewhere. The majority of the southern boundary nodes are assigned prescribed head for the Gordon Aquifer, "lower" aquifer zone, and "upper" aquifer zone (which includes transmissive zone, "AA" horizon, "A" horizon, and surface soil) with no-flow elsewhere. Figure 5.6 shows the vertical boundary conditions.

In order to estimate the Gordon Aquifer prescribed heads, two-dimensional kriging of digitized head contours from Aadland et al. (1995, Plate 42) and average hydraulic head at two monitoring wells (P-20B and RCP-1A) is performed. Figure 5.7 shows the estimated Gordon Aquifer head. The shaded circles represent the digitized head contours and the two monitoring wells.

Due to the lack of data in the "lower" aquifer zone, the estimated water table is translated and smoothed to form an estimate of the "lower" aquifer zone head. Figure 5.8 shows the estimated "lower" aquifer zone head and the shaded circle represents the monitoring well P-20C. A linear translation function is defined using the following information:

- Ground surface elevation at P-20C $=290 \mathrm{ft}$

- $\quad$ Estimated water table elevation at P-20C $=269 \mathrm{ft}$

- "lower" aquifer zone average head at P-20C $=238 \mathrm{ft}$

The translation function has the following form:

$$
\mathrm{H}_{\mathrm{laz}}=\mathrm{H}_{\mathrm{wT}}-\mathrm{T}(\mathrm{z}) \text {, at a given (Easting, Northing), }
$$

where $H_{\text {laz }}$ is the "lower" aquifer zone head, $H_{W T}$ is the estimated water table, $T$ is the translation in feet, and $T(z)$ is a linear function of ground elevation $z$. From the information at $P=20 C$, the linear function $T$ satisfies

$$
\mathrm{T}(290)=31 \mathrm{ft}, \text { since } 238=269-31\left(\mathrm{H}_{\mathrm{azz}}=\mathrm{H}_{\mathrm{Wr}}-\mathrm{T}(\mathrm{z})\right)
$$

In order to account for a small upward hydraulic gradient across the "tan clay" confining zone in low lying drainage areas, the linear function $\mathrm{T}$ satisfies

$$
\mathrm{T}(190)=-1 \mathrm{ft}
$$

The final estimated "lower" aquifer zone head results from a smoothing of $\mathrm{H}_{\mathrm{laz}}$. A common weighting scheme for producing a smooth estimate is given by Davis (1986):

$$
\hat{h}_{i, j}=\frac{1}{16}\left(h_{i-1, j-1}+h_{i-1, j+1}+h_{i+1, j-1}+h_{i+1, j+1}\right)+\frac{1}{8}\left(h_{i-1, j}+h_{i+1, j}+h_{i, j-1}+h_{i, j+1}\right)+\frac{1}{4} h_{i, j}
$$

where $h_{i, j}$ is the pre-smoother heads and $\hat{h}_{i, j}$ is the smoothed heads. The smoothing iteration defined above is performed eight times starting with $\mathrm{H}_{\mathrm{laz}}$ and produces the estimated "lower" aquifer zone head used for the prescribed head boundary conditions. 
In order to assign the prescribed head boundary conditions for the "upper" aquifer zone, twodimensional kriging of the digitized regional water table map onto a uniform grid is performed. Figure 5.1 shows the estimated water table elevation.

\subsection{CONDUCTIVITY FIELD}

The mini-permeability, laboratory permeability, mud fraction, and CPT data presented in Section 3 are correlated to horizontal conductivity $\left(\mathrm{K}_{\mathrm{h}}\right)$ and vertical conductivity $\left(\mathrm{K}_{\mathrm{v}}\right)$. The resulting conductivity data at borehole or CPT locations presented in Appendix F are segregated by elemental layer and averaged vertically to form scattered $2 \mathrm{D}$ conductivity data. Arithmetic averaging is used for horizontal conductivity. Arithmetic, harmonic, or geometric averaging is used for vertical conductivity depending on the vertical resolution and model calibration considerations. With all conductivity data having been segregated by element layer, the scattered data for each layer are fit using a bicubic spline algorithm (WSRC, 1997a, page 17) with a 500 $\mathrm{ft}$ grid resolution. Element conductivity values are computed by evaluating the two-dimensional fit at the element centroid locations. The two-dimensional elemental conductivity fields are stacked to form the three-dimensional $\mathrm{K}_{\mathrm{h}}$ and $\mathrm{K}_{\mathrm{v}}$ fields. Figure 5.9 illustrates the conductivity field construction process. Notice that mud fraction data overwrites CPT data and the permeability data overwrites mud fraction and CPT data. Buildings 105-R and 186-R are modeled as low conductivity $\left(\mathrm{K}_{\mathrm{h}}=\mathrm{K}_{\mathrm{v}}=10^{-8} \mathrm{ft} / \mathrm{d}\right)$ down to an elevation of $270 \mathrm{ft}$ and $278 \mathrm{ft}$, respectively.

The above process is used to construct the initial conductivity field. The calibrated conductivity field results from scaling and restricting the initial conductivity field. Figures 5.10 through 5.17 show the following subset of the two-dimensional elemental calibrated conductivity fields: $K_{h}$ distribution for Gordon Aquifer, $\mathrm{K}_{v}$ distribution for Gordon Confining Unit, $\mathrm{K}_{\mathrm{h}}$ distribution for "lower" aquifer zone, $\mathrm{K}_{\mathrm{v}}$ distribution for "tan clay" confining zone, $\mathrm{K}_{\mathrm{h}}$ distribution for the

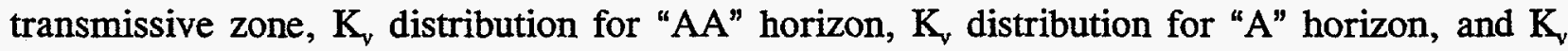
distribution for surface soil. Figure 5.15 through 5.17 show high $\mathrm{K}_{\mathrm{v}}$ near low lying areas, since the anisotropy ratio is restricted for the elements within $10 \mathrm{ft}$ of the surface. The maximum anisotropy ratio depends on the element thickness and is shown below.

\begin{tabular}{|c|c|}
\hline Element Thickness Range (ft) & Maximum Anisotropy Ratio \\
\hline$[0,0.5)$ & 1 \\
\hline$[0.5,1.5)$ & 3 \\
\hline$[1.5,2.5)$ & 10 \\
\hline$[2.5,3.5)$ & 30 \\
\hline$[3.5,4.5)$ & 100 \\
\hline
\end{tabular}

Figures 5.18 and 5.19 show a cross-section of the $\mathrm{K}_{\mathrm{h}}$ and $\mathrm{K}_{\mathrm{v}}$ conductivity fields, respectively, at the $25^{\text {th }}$ north-south nodal plane. 


\subsubsection{Mini-Permeability and Laboratory Permeability}

The mini-permeability data are used as horizontal conductivity data with $\mathrm{K}_{\mathrm{r}}$ being defined by an anisotropy ratio depending on the hydrostratigraphic unit. Laboratory permeability data are specified as $K_{h}$ or $K_{v}$. For soil samples that do not have both $K_{h}$ and $K_{v}$ estimates, an anisotropy ratio is used to produce the pair of conductivity estimates. The mini-permeability and laboratory permeability anisotropy ratios were initially set equal to 3.6 following WSRC (1997a, page 16).

\subsubsection{Mud Fraction Correlation}

For each hydrostratigraphic unit, a correlation is defined between mud fraction and $K_{h}$ with $K_{v}$ being defined by an anisotropy ratio. The mud fraction to $\mathrm{K}_{\mathrm{h}}$ correlation is a combination of the correlation presented by Celeste (1997) and the correlation presented by WSRC (1997c, Figure 5-2) which was prepared by Parsons Engineering Science, Inc. Both correlations relate laboratory permeability measurements and mud fraction. The Celeste correlation fits mostly high conductivity data, while the Parsons correlation fits more low conductivity data. The Celeste correlation is

$$
\log \left(\mathrm{K}_{\mathrm{h}}(\mathrm{ft} / \mathrm{d})\right)=1.86-(4.01 * \text { mud fraction }),
$$

with $\mathrm{K}_{\mathrm{h}}$ varying from $0.0071 \mathrm{ft} / \mathrm{d}$ to $72.4 \mathrm{ft} / \mathrm{d}$.

The Parsons correlation is approximated by a piecewise linear function interpolating the following points:

\begin{tabular}{|c|c|}
\hline Mud Fraction & Parson Fit $(\log (\mathrm{Kh}(\mathrm{ft} / \mathrm{d})))$ \\
\hline 0.13 & -1.55 \\
\hline 0.25 & -2.55 \\
\hline 0.46 & -3.55 \\
\hline 0.82 & -4.55 \\
\hline
\end{tabular}

The Parsons $\mathrm{K}_{\mathrm{h}}$ varies from $2.8 \times 10^{-5} \mathrm{ft} / \mathrm{d}$ to $0.028 \mathrm{ft} / \mathrm{d}$. The mud fraction to conductivity anisotropy ratios were initially set equal to 3.6 following WSRC (1997a, page 16).

\subsubsection{CPT Correlation}

The CPT to $\mathrm{K}_{\mathrm{h}}$ correlation is a combination of two CPT regressions developed by Celeste (1997). The CPT regression for sandy sediments (CPTSS) is given by:

$$
\begin{gathered}
\log \left(\mathrm{K}_{\mathrm{h}}(\mathrm{ft} / \mathrm{d})\right)=0.4902+(0.0454 * \text { friction ratio }) \\
+(0.0896 * \text { pore pressure })-(0.0849 * \text { sleeve stress }) \\
+(0.00538 * \text { tip resistance })
\end{gathered}
$$

The CPT regression for muddy sediments (CPTMS) is given by:

$$
\log \left(\mathrm{K}_{\mathrm{h}}(\mathrm{ft} / \mathrm{d})\right)=-3.59-(0.104 * \text { sleeve stress })+(0.0212 * \text { tip resistance })
$$


Celeste (1997) suggested using CPTSS regression when the friction ratio is less than 5.5 and ARA's pore pressure is less than 20. Otherwise, use the CPTMS regression. The pore pressure clause for ARA's data is for the Gordon Aquifer Confining Unit which has high pore pressure, but low friction ratios. The CPTSS $\mathrm{K}_{\mathrm{h}}$ varies from $0.4 \mathrm{ft} / \mathrm{d}$ to $100 \mathrm{ft} / \mathrm{d}$ and the CPTMS $\mathrm{K}_{\mathrm{h}}$ varies from $3.3 \times 10^{-5} \mathrm{ft} / \mathrm{d}$ to $0.09 \mathrm{ft} / \mathrm{d}$. In order to smooth the transition between the two regressions, a friction ratio transition interval [ratlower, ratupper] is defined. Initially, [ratlower, ratupper] $=[3.0,5.5]$. ARA CPT data with pore pressure greater than 20 , use the CPTMS regression. The remaining CPT data use the following:

$$
\log \left(\mathrm{K}_{\mathrm{h}}\right)=\left\{\begin{array}{l}
\text { CPTSS if friction ratio < ratlower } \\
\text { CPTSS }+\left(\text { CPTMS }- \text { CPTSS) } \frac{\text { (friction ratio - ratlower) }}{\text { (ratupper - ratlower) }} \text { if ratlower } \leq\right. \text { friction ratio } \\
\text { CPTMS if friction ratio > ratupper }
\end{array}\right.
$$




\subsection{GROUNDWATER FLOW MODEL RESULTS}

The FACT code (WSRC, 1995) was used with the finite-element option and Newton-Raphson method with one backtrack. The "pseudo-soil" relative permeability and water retention curves were used to model flow in the unsaturated zone. For most calibration runs, the FACT code needed 8 iterations to converge with a restart file and 20 minutes of CPU on a SGI R8000.

\subsection{MODEL CALIBRATION}

The monitoring well average head data were used as targets for calibrating the R-Reactor Area model. Initial calibration runs had perched water in the " $\mathrm{A}$ " horizon and surface soil and large negative head residuals (simulated-data) in the "AA" horizon. This was improved by increasing $\mathrm{K}_{\mathrm{v}}$ in the "A" horizon and decreasing $\mathrm{K}_{\mathrm{v}}$ in the "tan clay" confining zone. Since the conductivity field was constructed from several different types of data, the initial conductivity field had large spatial variability which caused the simulated heads to have more variability than the monitoring well data. Several calibration runs were performed to smooth the conductivity field. A detailed listing of all calibration runs is presented in Appendix G. Since hydrostratigraphic unit boundaries exhibit large variation in elevation north of RRSB, water table elevations for all calibration runs were not as smooth as the estimated water table in Figure 5.1. The hydrostratigraphic "picks" should be reexamined by WSRC. Listed below is a summary of the last calibration run (Run 9C).

1. Geometric and arithmetic averaging were used to vertically average the $\mathrm{K}_{\mathrm{v}}$ data in " $\mathrm{A}$ " horizon. Harmonic averaging was used elsewhere.

2. Correlations between mud fraction and $\mathrm{K}_{\mathrm{h}}$ are given in Table 6.1.

3. Friction ratio transition intervals for the CPT correlations are given in Table 6.2. In order to be consistent with the Fugro CPT data, ARA friction ratios were increased by one unit.

4. Mini-permeability anisotropy ratios are given in Table 6.3.

5. RCP-1A mud fraction data is consistently lower than surrounding boreholes in surface soil, "A" horizon, and "AA" horizon. RCP-1A mud fraction data above the transmissive zone was not used.

6. Three calibration zones were defined north of RRSBs which were designed to raise the water table in the area. Figure 6.1 shows the calibration zones and Table 6.4 presents the restrictions on the conductivity for the three calibration zones.

7. The final modifications to the initial conductivity field are specified in Table 6.5.

Table 6.6 presents the calibrated model average conductivities for each hydrostratigraphic unit. After the final calibration run (Run 9C) was made, a Pen Branch fault sensitivity run (Run 9D) was performed. South of Pen Branch fault was lowered to match north of Pen Branch fault, which resulted in lower hydraulic head at the monitoring wells in all hydrostratigraphic units (see Appendix G). The lower heads are due to higher transmissibility in the 'lower' aquifer zone and transmissive zone south of R-Reactor Area. 


\subsection{HEAD RESIDUALS}

Simulated head and residual at the 55 monitoring wells is presented in Appendix A for Run 9C. The overall root-mean-squared (RMS) is $3.7 \mathrm{ft}$ and Table 6.7 shows the average residual (simulated-data) and RMS for each unit. Figures 6.2 through 6.5 show areal views of the head residuals by hydrostratigraphic unit. Figure 6.3 shows three large negative residuals (simulated head is low) in the "AA" horizon surrounding RRSBs and one large positive residual north of RRSB. Figure 6.4 shows the "A" horizon head residuals. Several positive residuals are located north of RRSBs. The RRSBs contain one large negative residual. Several large negative residual are located inside the R-Reactor Area fence. All residuals south of the R-Reactor Area fence are positive. The three monitoring wells constructed in 1997 (one time measurements) are located east of the R-Reactor Area and all three have a large positive residual. Figure 6.5 presents the surface soil head residuals. All residuals are negative. Monitoring well RSC-8 has a head residual of -9.6 $\mathrm{ft}$.

\subsection{GROUNDWATER RECHARGE/DISCHARGE}

The average recharge rate over the total model area is $16.2 \mathrm{in} / \mathrm{yr}$ and the local recharge rate away from discharge areas is $18 \mathrm{in} / \mathrm{yr}$. Figure 6.6 illustrates the rate of groundwater recharge/discharge for the R-Reactor Area model. Table 6.8 compares stream baseflow estimates from Hiergesell (1997) to simulated values and Figure 6.7 presents the discharge zones listed in Table 6.8. Considering the uncertainty of baseflow estimates, the agreement between measured and simulated baseflow is acceptable.

\subsection{GROUNDWATER FLOW PATTERNS}

Figures 6.8 through 6.10 illustrate simulated head of the Gordon Aquifer (nodal layer 2), "lower" aquifer zone (nodal layer 6), and transmissive zone (nodal layer 11), respectively. Figure 6.11 presents the simulated water table. Notice that the drainage areas and the R-effluent canal have affected the water table. The water table is highest at the RRSBs because of the low $K_{v}$ in " $A$ " horizon (Figure 5.17) and surface soil (Figure 5.18). The northern edge of the water table mound at the RRSBs follows the contours of the hydrostratigraphic units (Figures 4.10, 4.11, and 4.12) Figure 6.12 shows a cross-section of the simulated head at the $25^{\text {th }}$ north-south nodal plane. Figure 6.13 presents a cross-section of the simulated water saturation at the $25^{\text {th }}$ north-south nodal plane, which crosses through the R-Reactor Area and RRSBs.

Further investigation of the groundwater flow requires three-dimensional pathline analysis. The flow field is seeded within $10 \mathrm{ft}$ of the ground surface at eight locations listed in Table 6.9. Figure 6.14 presents an areal views of the eight three-dimensional pathlines. Figures 6.15 and 6.16 present side views of the pathlines facing north and west, respectively. Particle tracing used an effective porosity of $25 \%$ (WSRC, 1997a, page 7).

Since there is a water divide northwest of the RRSBs, all seeds move eastward toward Pond C. Seed 1 traveled down to the transmissive zone before discharging to the surface. Seed 2 traveled down to the "lower" aquifer zone before leaving the side of the model. Seeds 3, 4, 5, and 7 traveled down to the transmissive zone before discharging to the surface. Seeds 6 and 8 traveled down to the Gordon Aquifer before leaving the bottom of the model. 
The pathlines for Seeds $4,5,6$, and 7 are subject to uncertainty due to the uncertainty of the location of the water divide north of RRSB. Changes in recharge, conductivity distribution or contours of the hydostratigraphic units may move or turn the water divide causing the pathlines to move northward toward Mill Creek. 


\subsection{CONCLUSIONS}

- A detailed groundwater flow model has been developed for the R-Reactor Area which simulates average flow conditions. The model incorporates Phase I hydrostratigraphic data, borehole data, CPT data, and laboratory permeability data. The simulated groundwater flow field is consistent with the observed water levels in wells and groundwater recharge and discharge estimates. Simulated hydraulic heads have an acceptable RMS deviation of 3.7 feet. This groundwater model provides a sound basis for subsequent detailed contaminant transport simulations.

- The groundwater flow model should be further refined using Phase II characterization and monitoring data, before contaminant transport simulations are performed. The Phase II characterization data will assist in resolving uncertainties by providing:

1. Head data in the "undefined high permeability zone" directly above the Tan Clay confining zone.

2. Additional CPT data will add to the lithologic and hydrostratigraphic "pick" data.

3. Additional information on the perched water table directly beneath the RRSBs. The Phase II characterization data should resolve the extent and thickness of the perched water table and its effects on the subsequent transport simulations.

Additional data will assist in the refinement of particle tracking from the RRSBs to the final discharge areas.

- Transient flow simulations should be considered to refine the model in the next phase. The installation of the asphalt liner has resulted in a decrease in hydraulic heads in monitoring wells in and about the RRSB and provides an opportunity for transient calibration. 


\subsection{REFERENCES}

Aadland, R.K., Gellici, J.A. and Thayer, P.A., 1995. Hydrogeologic framework of west-central south Carolina. South Carolina Department of Natural Resources, Water Resources Division Report 5.

Aadland, R. K., M. K. Harris, C. M. Lewis, T. F. Gaughan, and T. M. Westbrook, 1991, Hydrostratigraphy of the General Separations Area, Savannah River Site (SRS), South Carolina, USDOE Report WSRC-RP-91-13, Westinghouse Savannah River Company, Aiken, SC 29808, $114 \mathrm{p}$.

Colquhoun, D. J., I. D. Woollen, D. S. Van Nieuwenhuise, G. G. Padgett, R. W. Oldham, D. C. Boylan, J. W. Bishop, and P. D. Howell, 1983, Surface and Subsurface Stratigraphy, Structure and Aquifers of the South Carolina Coastal Plain, SCDHEC Report ISBN 0-9613154-0-7, 78p.

Davis, J.C., 1986. Statistics and Data Analysis in Geology, $2^{\text {nd }}$ Edition, John Wiley \& Sons, New York.

Dennehy, K. F., D. C. Prowell, and P. B. McMahon, 1989, Reconnaissance Hydrogeologic Investigation of the Defense Waste Processing Facility and Vicinity, Savannah River Plant, South Carolina, U. S. Geological Survey Water Resources Investigations Report 88-4221, 68p.

Fallaw, W.C. and Price, V., 1995. Stratigraphy of the Savannah River site and vicinity. Southeastern Geology, 35: 21-58.

Fallaw, W. C., V. Price, and P. Thayer, 1990, "Stratigraphy of the Savannah River Site, South Carolina", in Zullo, V. A., W. B. Harris, and V. Price, eds., 1990, Savannah River Region: Transition Between the Gulf and Atlantic Coastal Plains. Proceedings of the Second Bald Head Island Conference on Coastal Plains Geology, University of North Carolina at Wilmington, Wilmington, N.C.

Hamming, R.W., 1973. Numerical Methods for Scientists and Engineers, Dover Publications, Inc., New York.

Harris, M. K., R. K. Aadland, and T. M. Westbrook, 1990, "Lithological and Hydrological Characteristics of the Tertiary Hydrostratigraphic Systems of the General Separations Area, Savannah River Site, South Carolina," in Zullo, V. A., W. B. Harris, and V. Price, eds., 1990, Savannah River Region: Transition Between the Gulf and Atlantic Coastal Plains. Proceedings of the Second Bald Head Island Conference on Coastal Plains Geology, University of North Carolina at Wilmington, Wilmington, N.C., pp. 68-73.

Hiergesell, R.A., 1997. Personal Communication, June 17.

Logan, W.R. and Euler, G.M., 1989. Geology and groundwater resources of Allendale, Bamberg, and Barnwell counties and part of Aiken county, South Carolina. South Carolina Water Resources Commission Report, 155, 113 pp. 
Pekkala, R.O., Jewell, C.E., Price, V., and Bledsoe, H.W., 1987. Environmental Information Document: L-Area Oil and Chemical Basin, DPST-85-700, E.I. du Pont de Nemours and Company, Savannah River Plant, Aiken, SC.

WSRC, 1995. FACT: Subsurface Flow and Contaminant Transport Documentation and User's Guide (U), WSRC-TR-95-0223, Westinghouse Savannah River Company Aiken, SC.

WSRC, 1997a. Integrated Hydrogeological Model of the General Separation Area (U), Volume 2: Groundwater Flow Model, WSRC-TR-96-0399, Westinghouse Savannah River Company, Aiken, SC.

WSRC, 1997b. Preliminary Characterization Report Phase I RFI/RI for the R-Reactor Seepage Basins/108-4R Overflow Basin (U), WSRC-RP-97-196, Westinghouse Savannah River Company, Aiken, SC.

WSRC, 1997c. Evaluation of Cone penetrometer Data for permeability Correlation at Savannah River Site, WSRC-RP-97-63, Westinghouse Savannah River Company, Aiken, SC. 


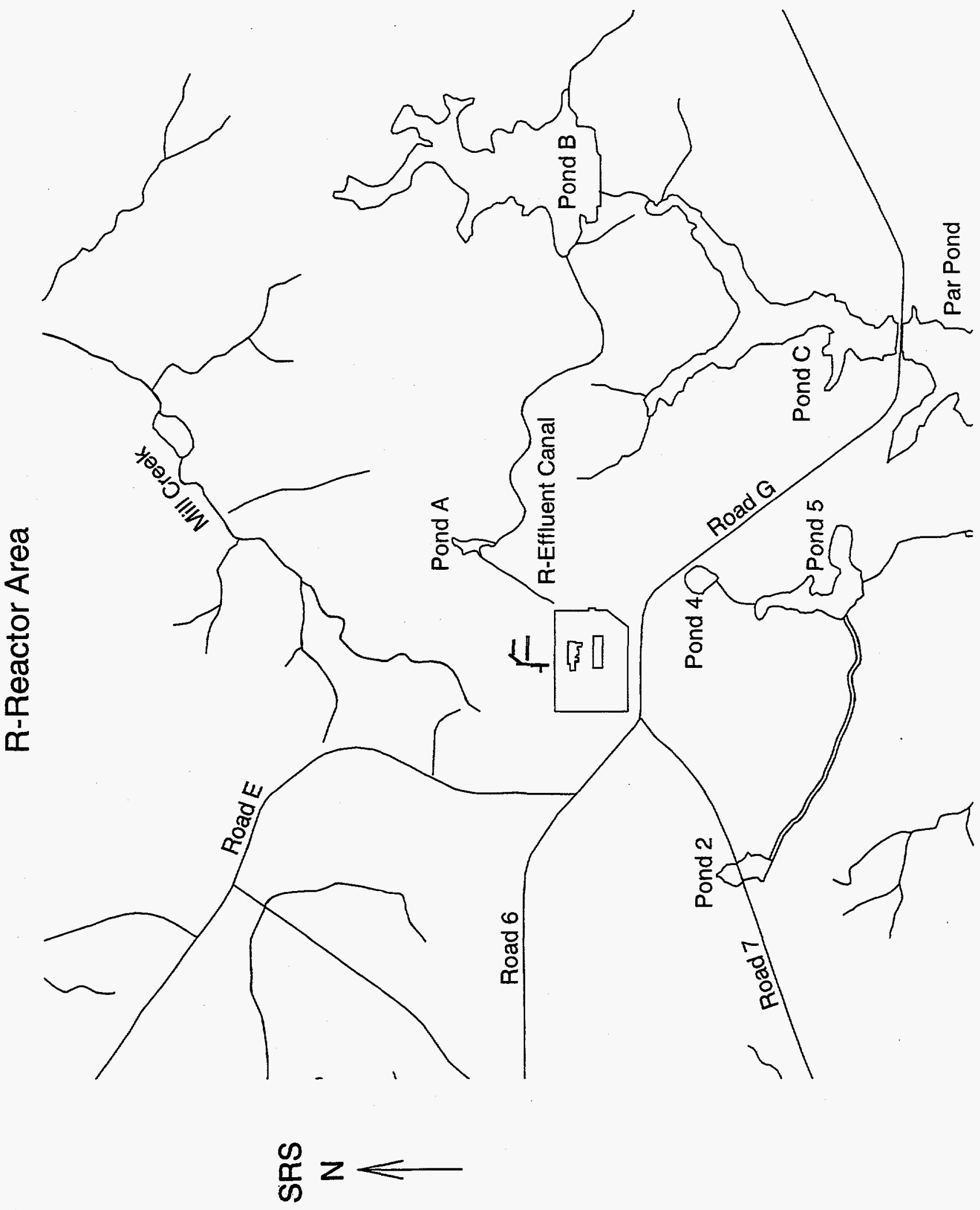

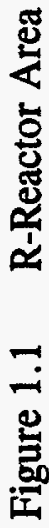




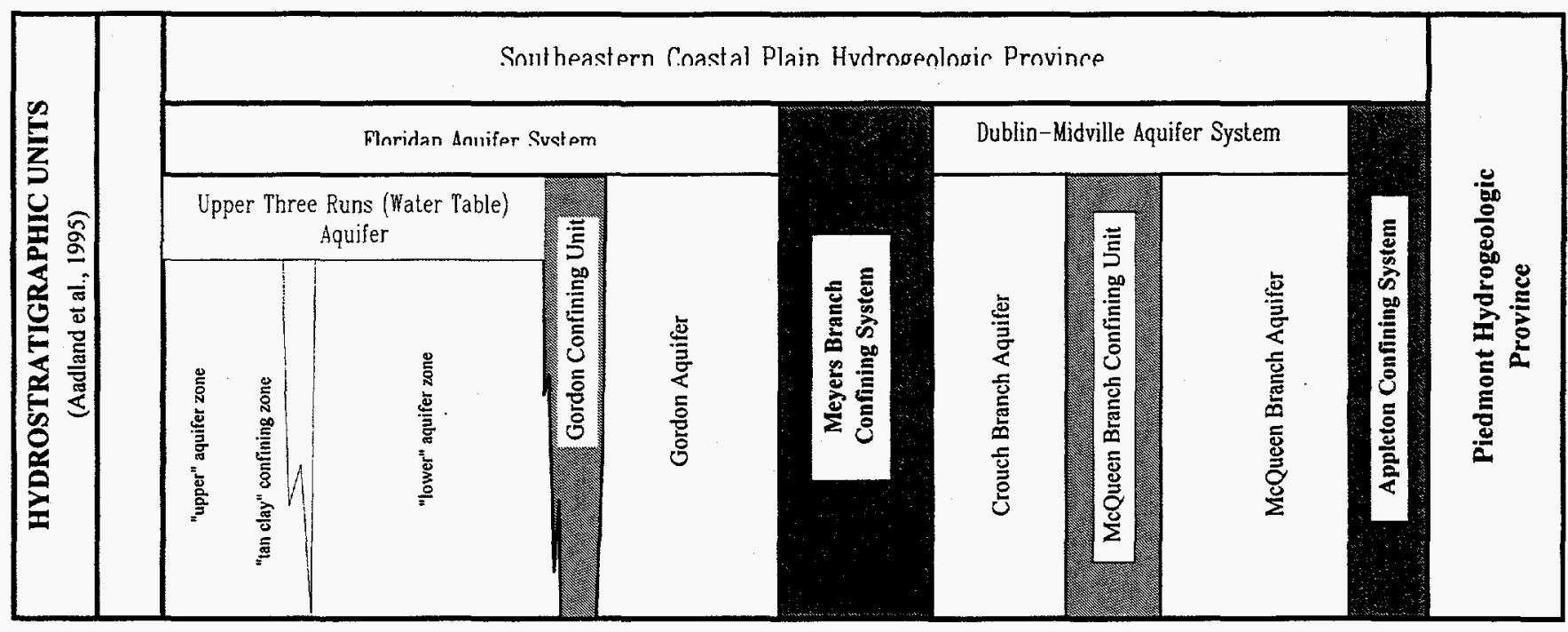

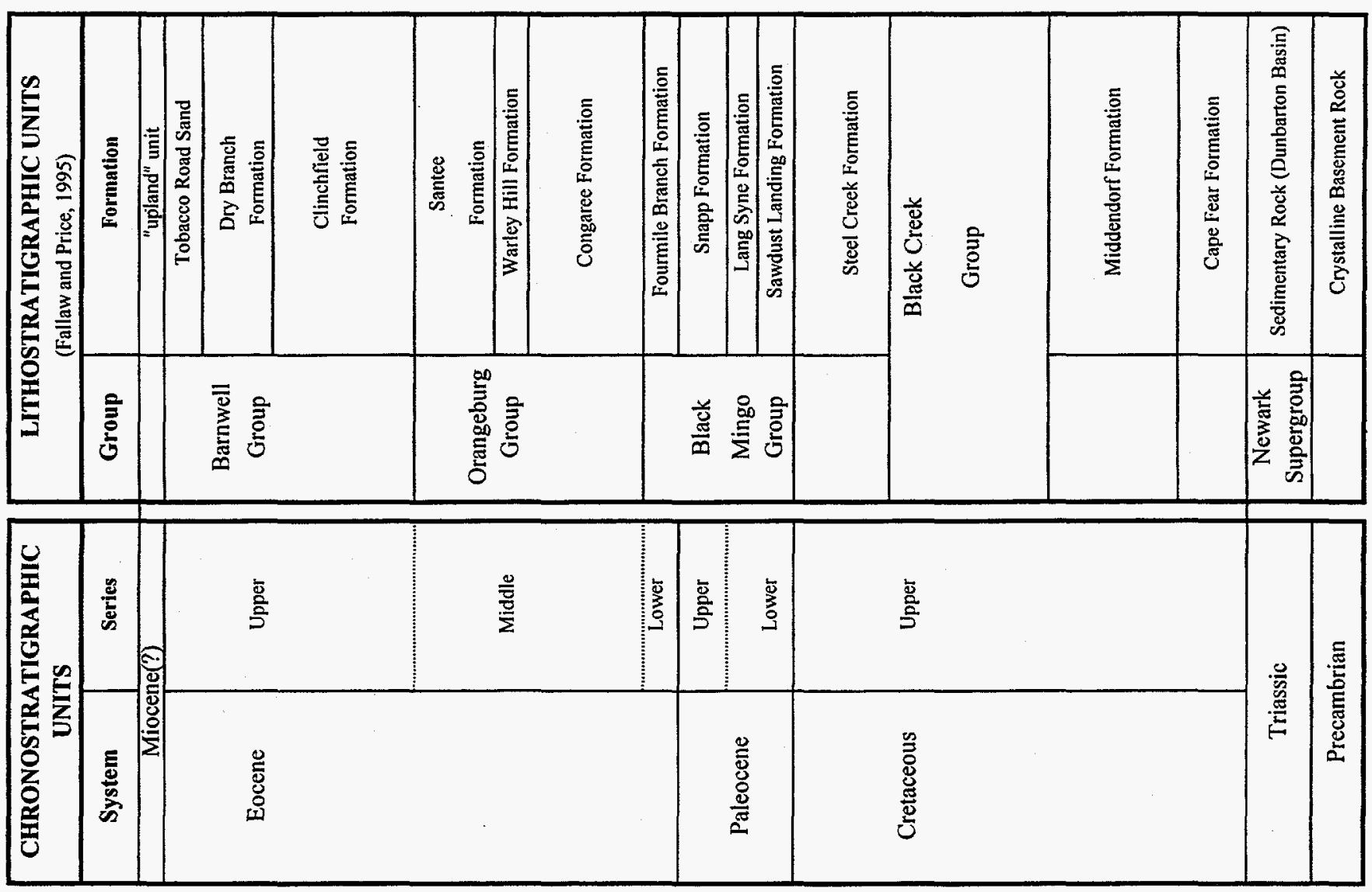

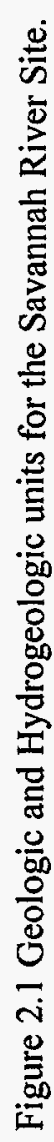




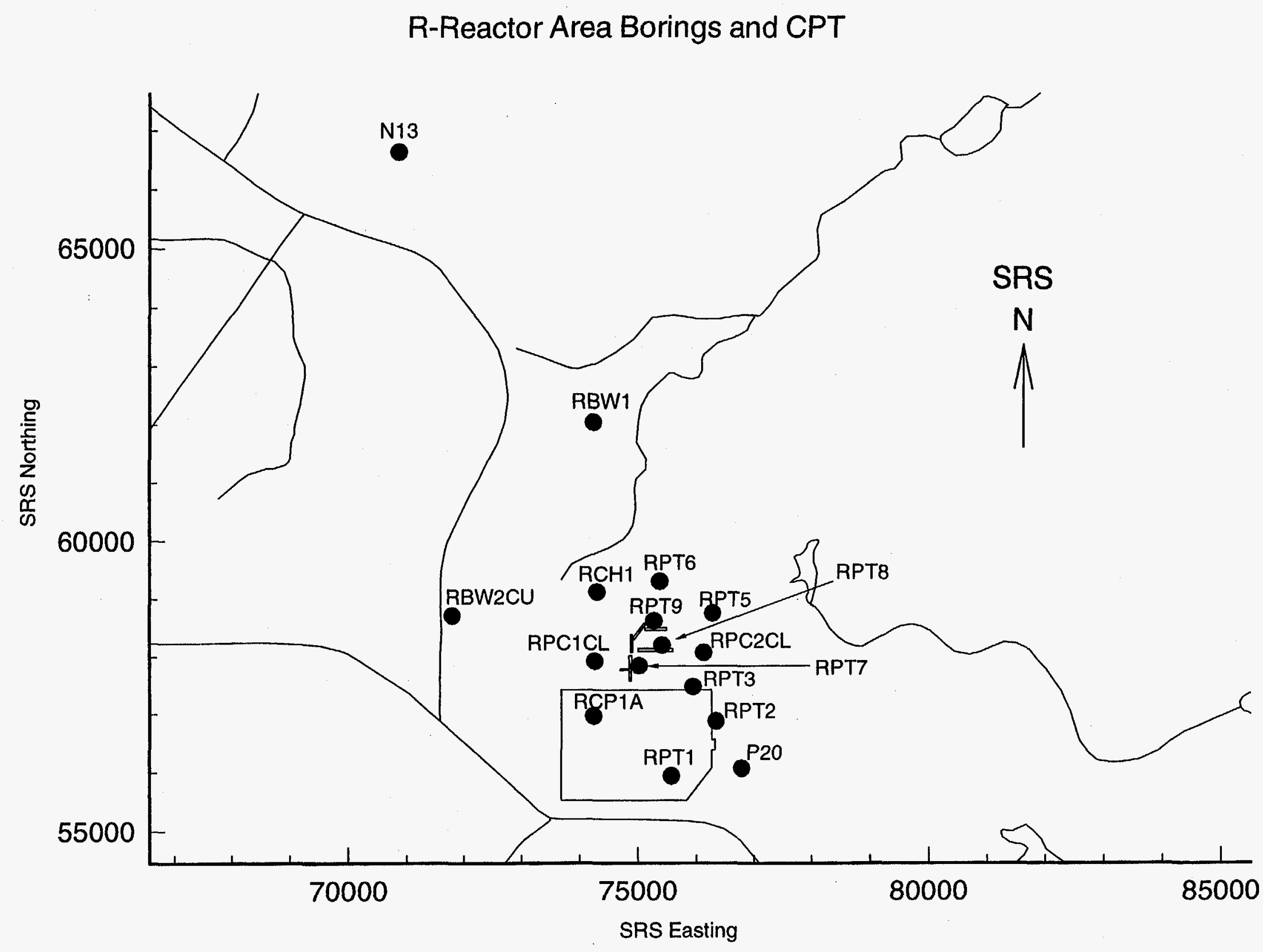

Figure 3.1 Name and Location of Borehole and CPT Data 


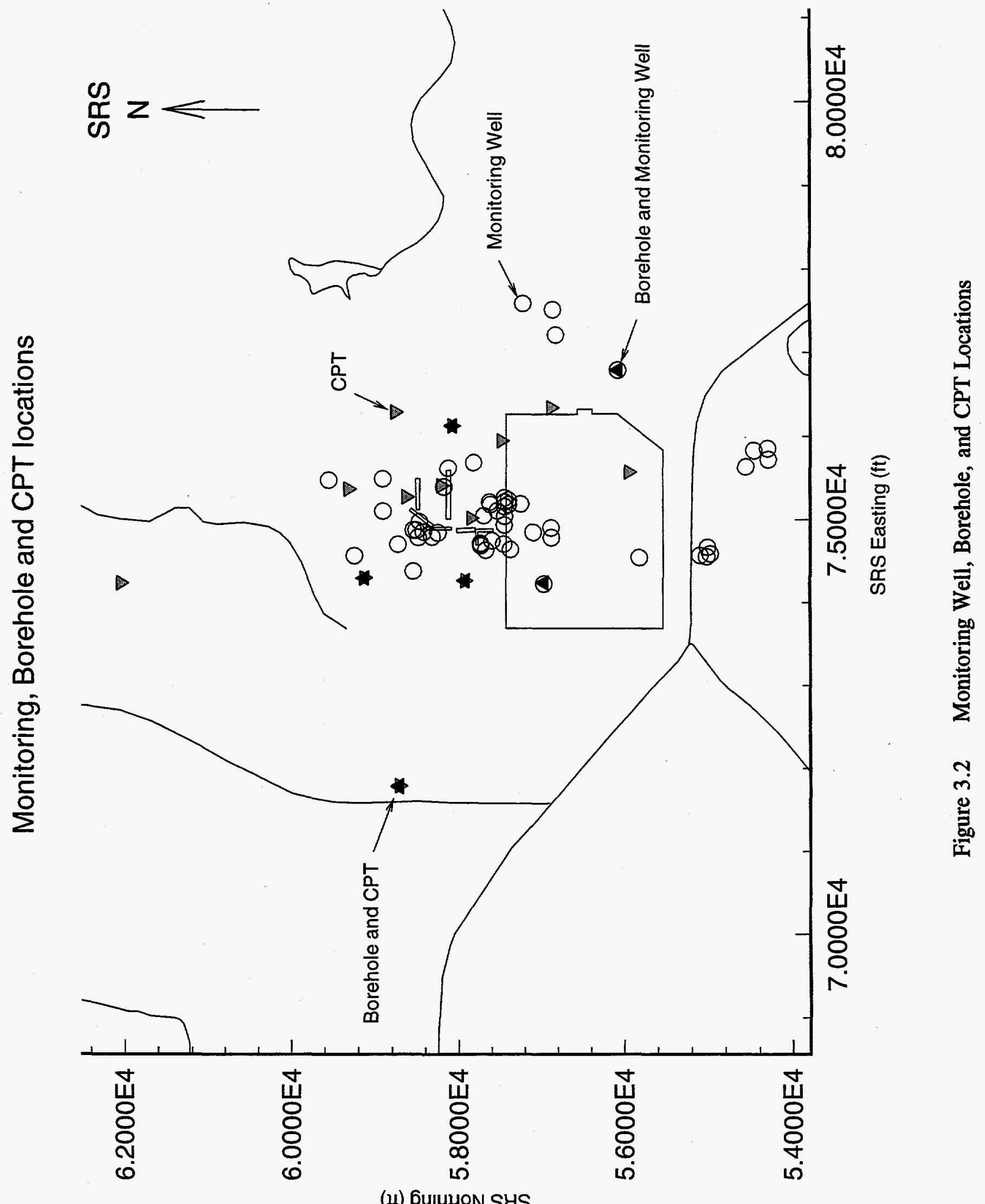




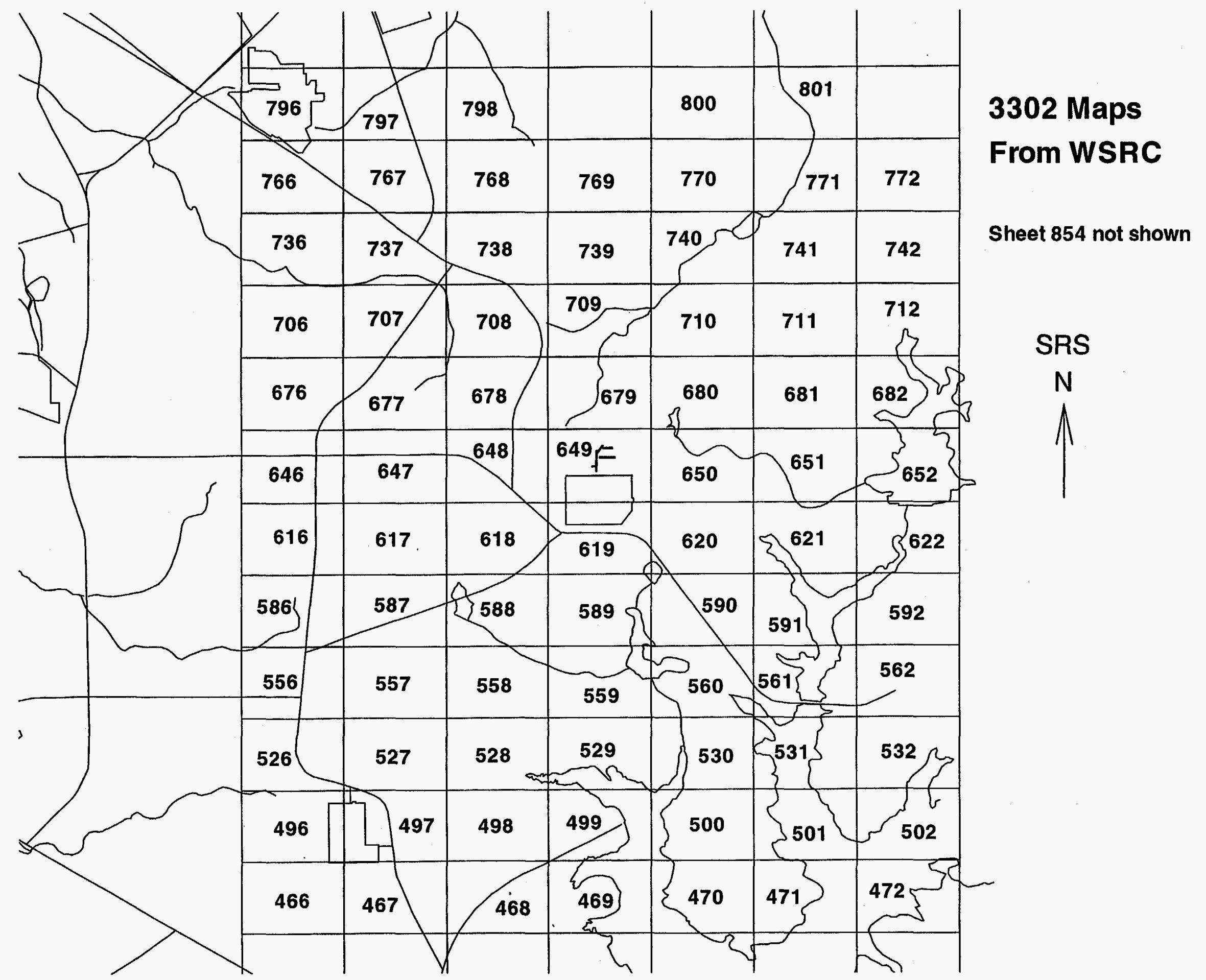

Figure 3.3 3302 Maps Obtained from WSRC 
Surface Elevation (ft)

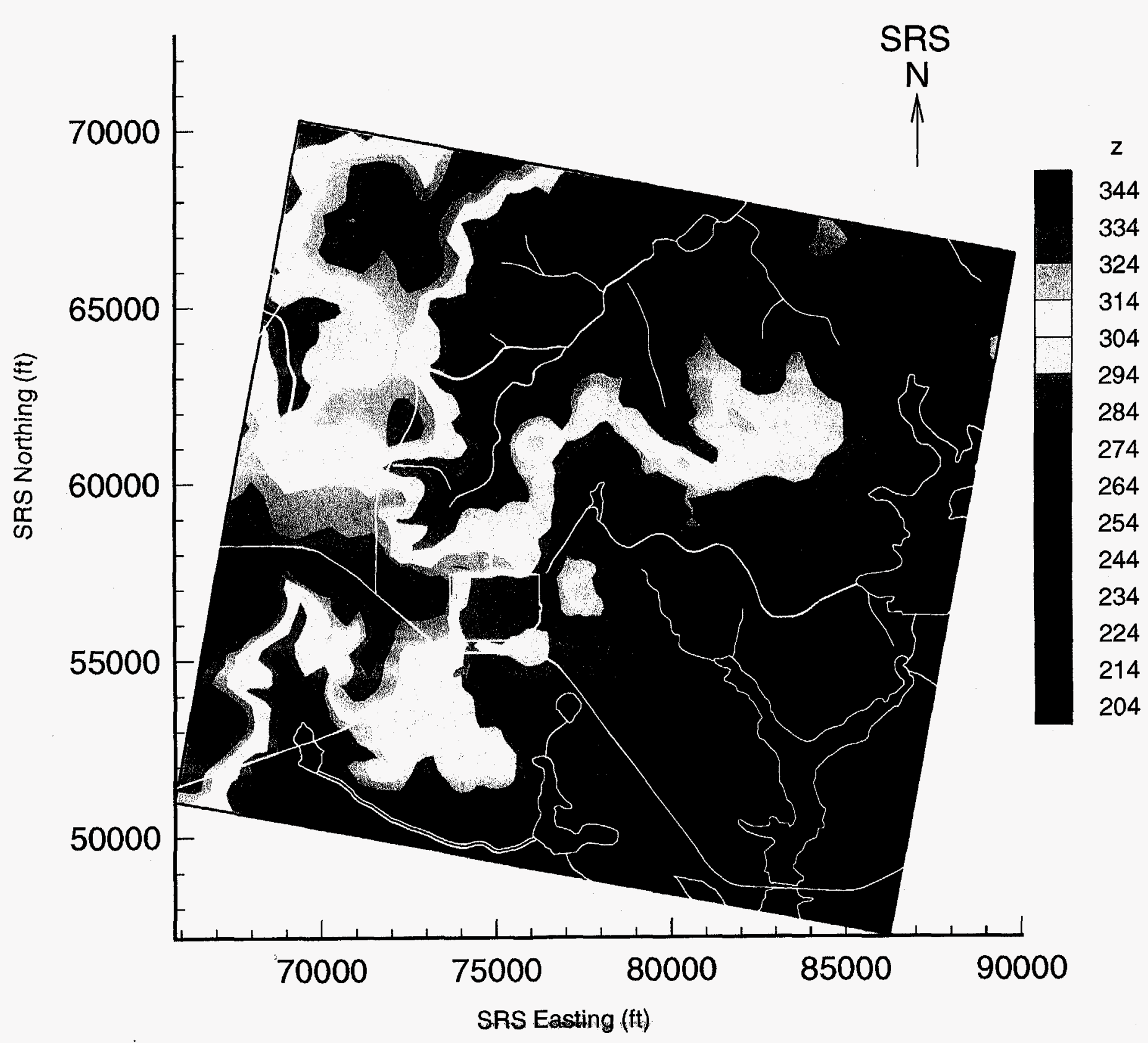


Model Area and Pen Branch Fault

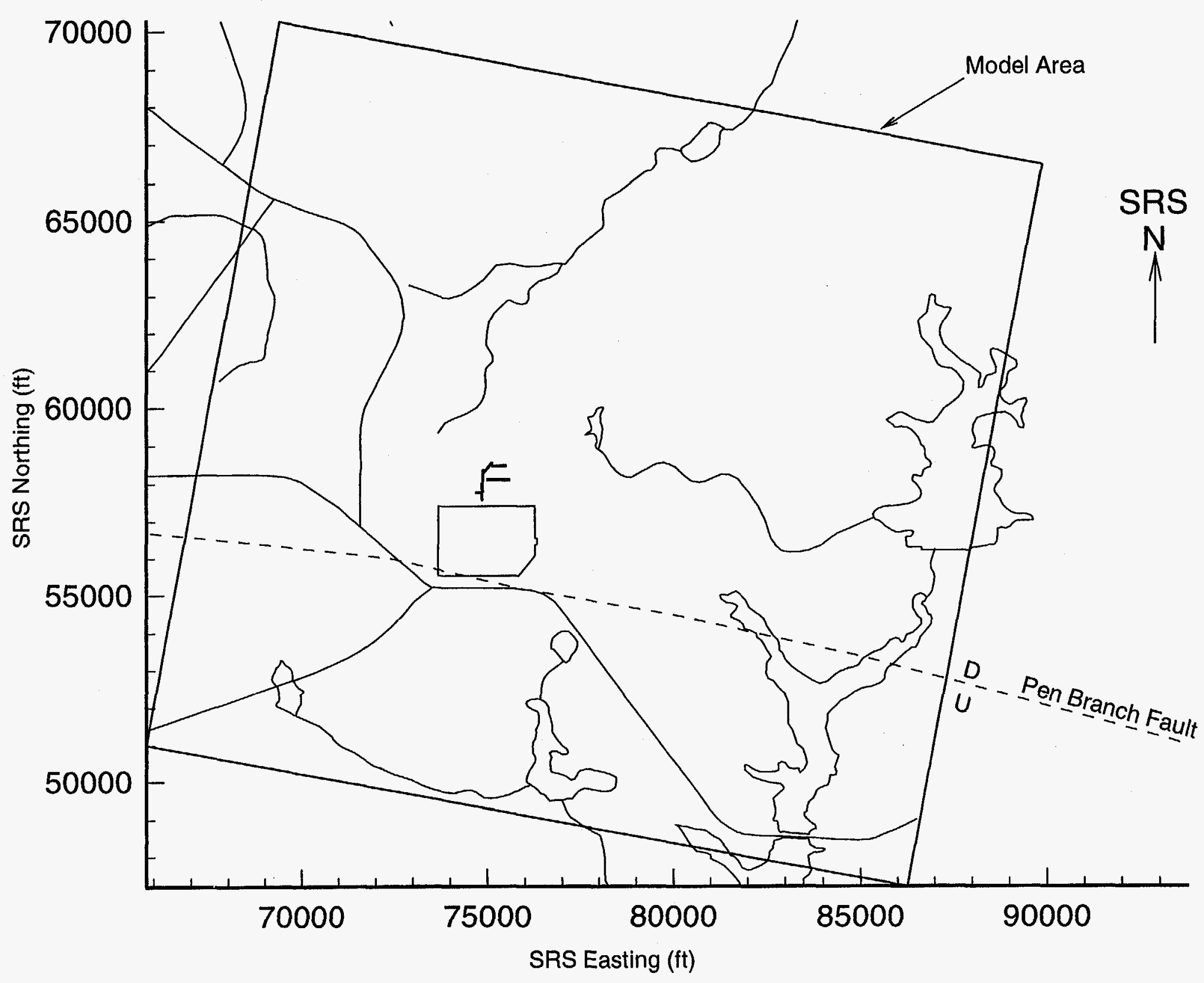

Figure 4.1 Model Area and Pen Branch Fault 


\section{Top of Crouch Branch Confining Unit (Prior to faulting) (ft)}

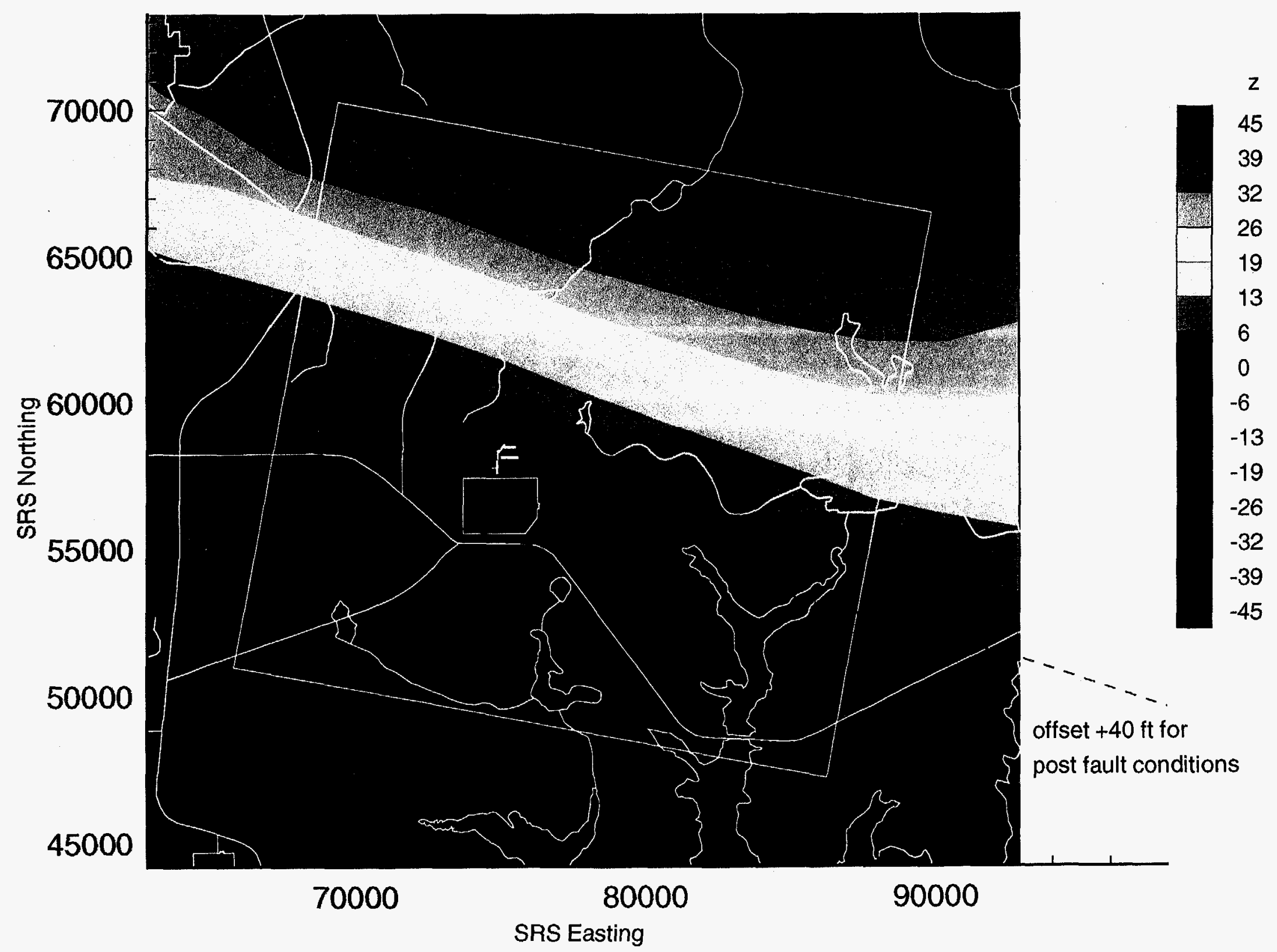

Figure 4.2 Top of Crouch Branch Confining Unit Surface Trend (Prior to faulting) 


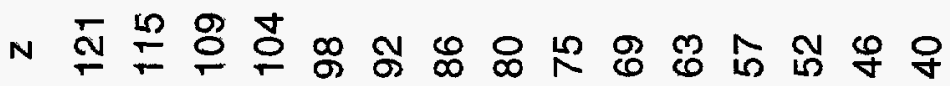

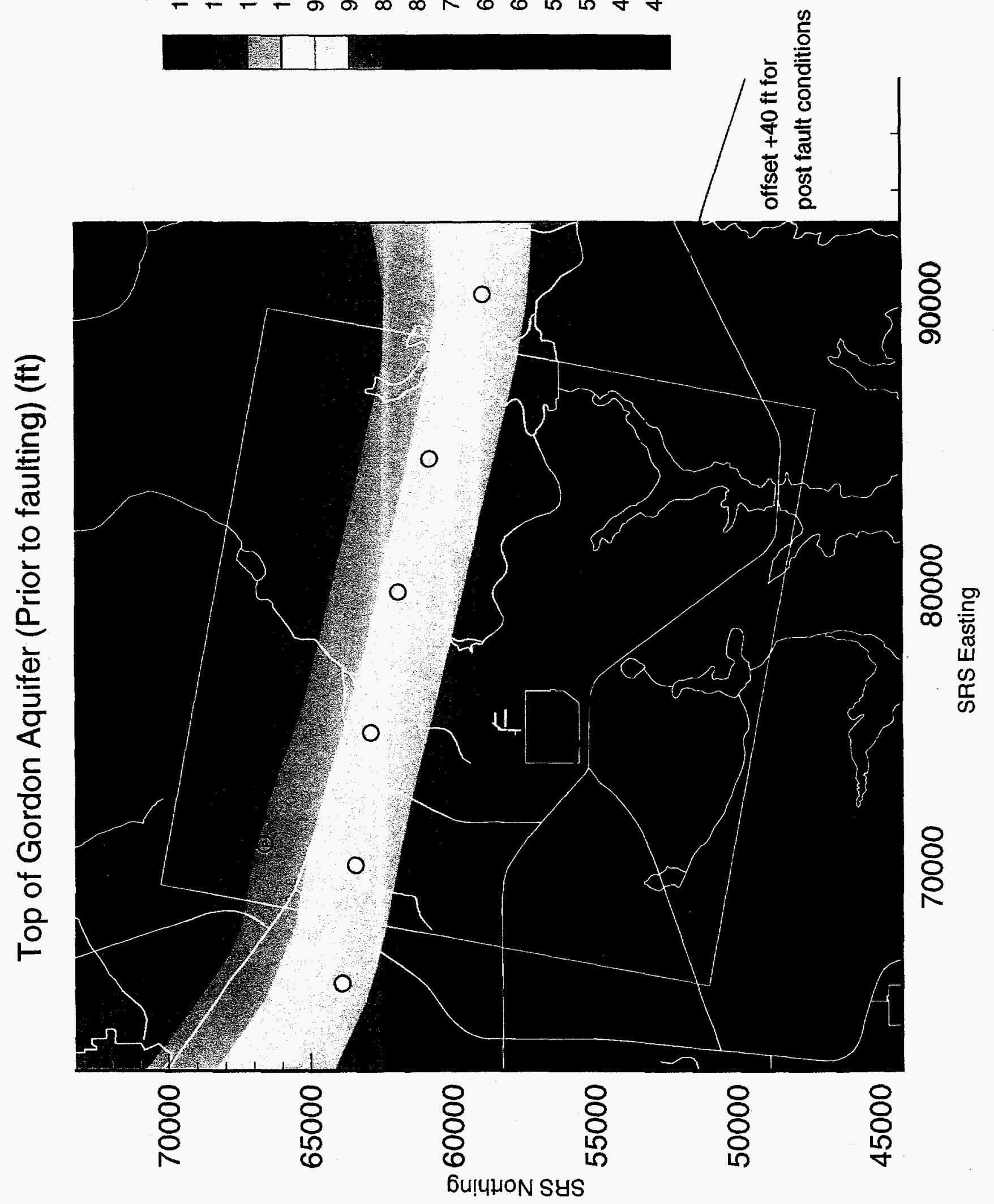




\section{N}

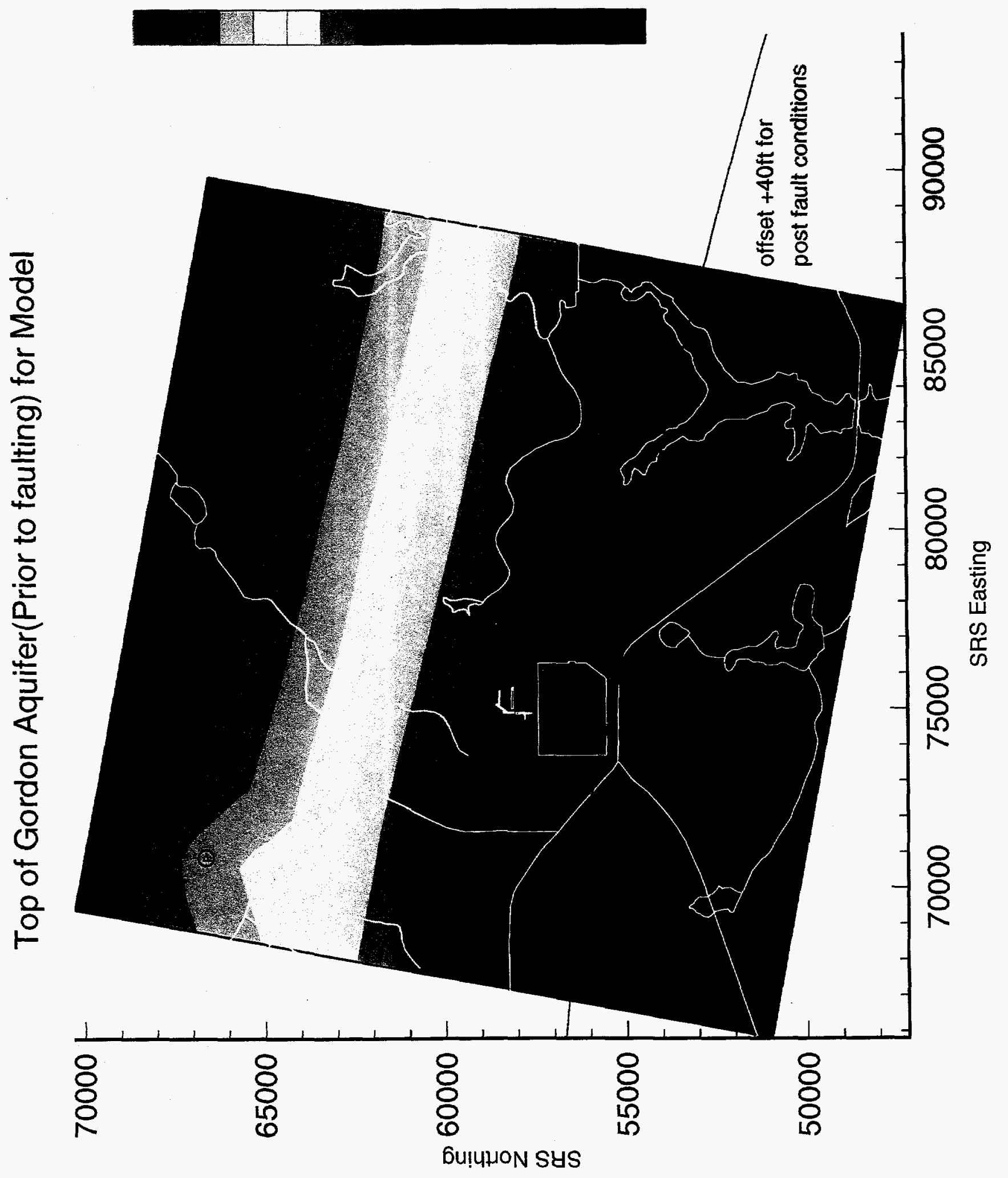




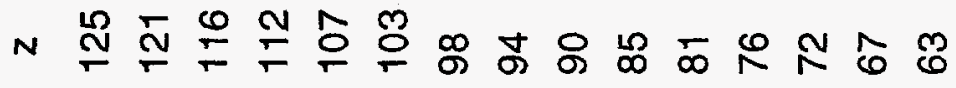

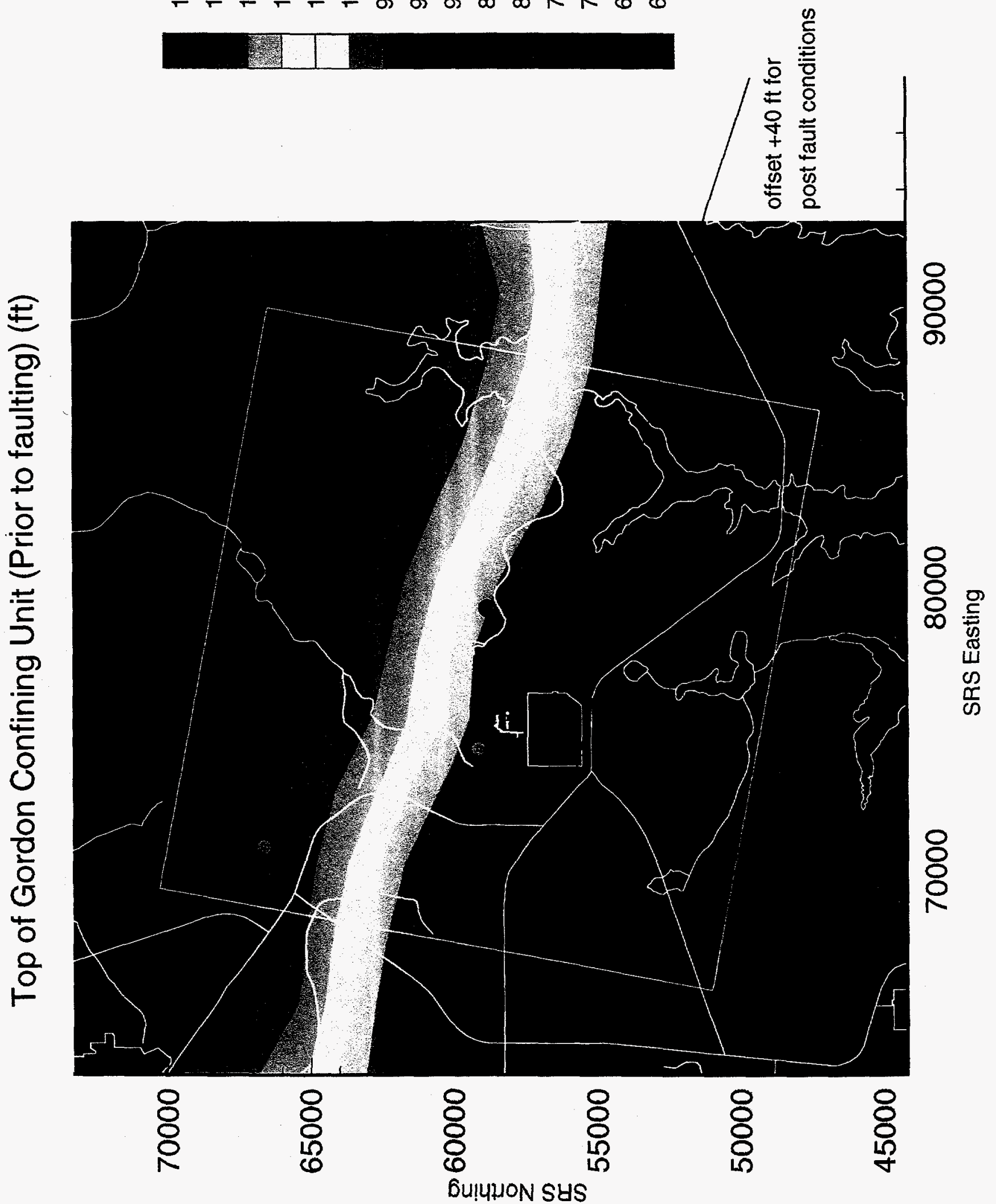




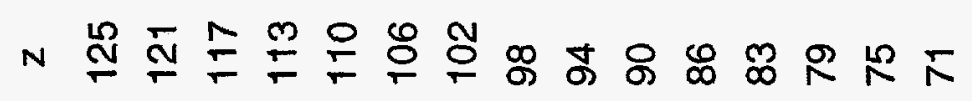

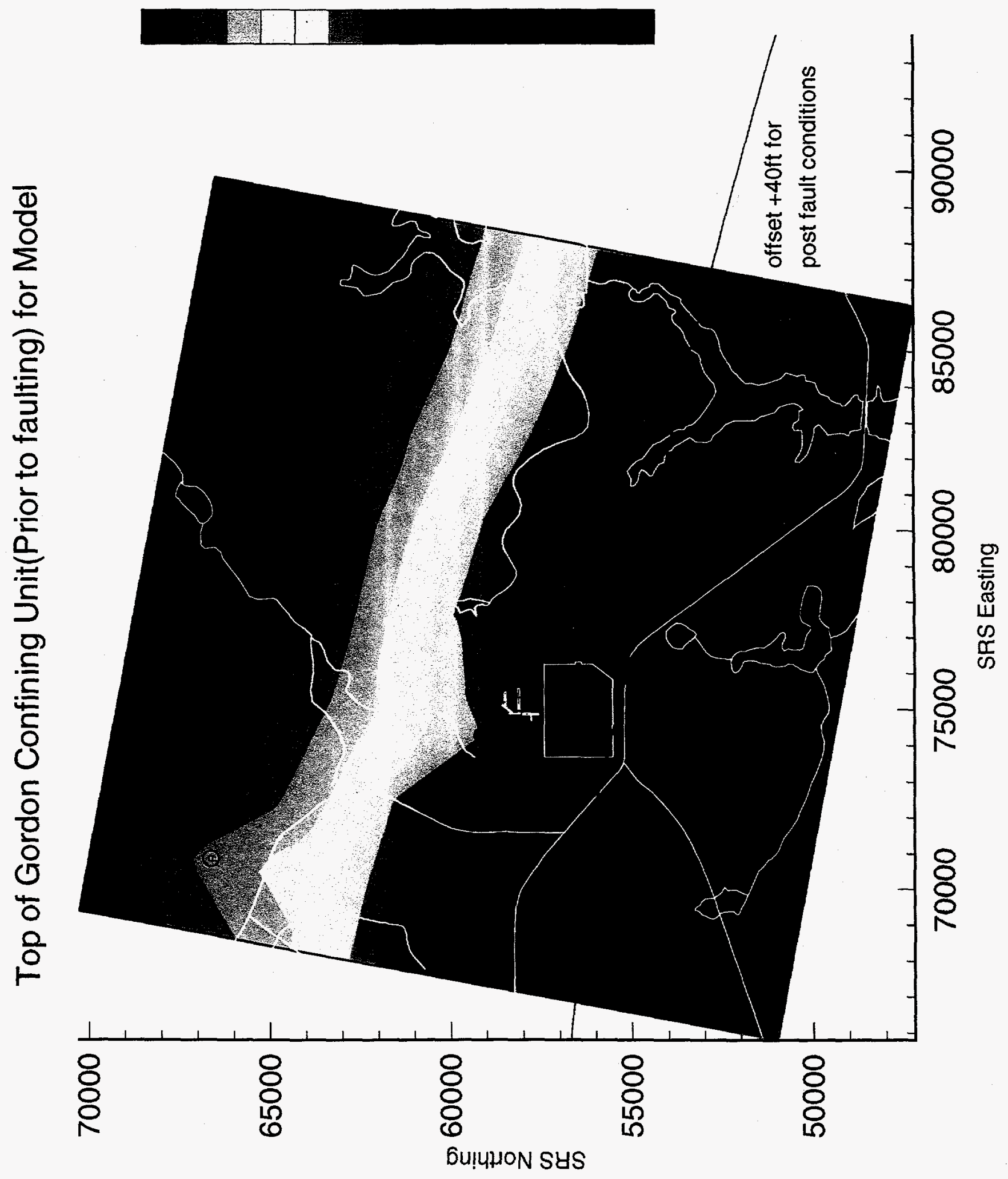

是 


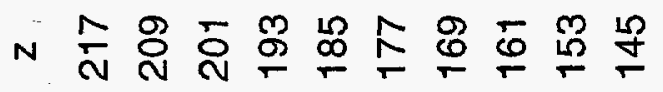

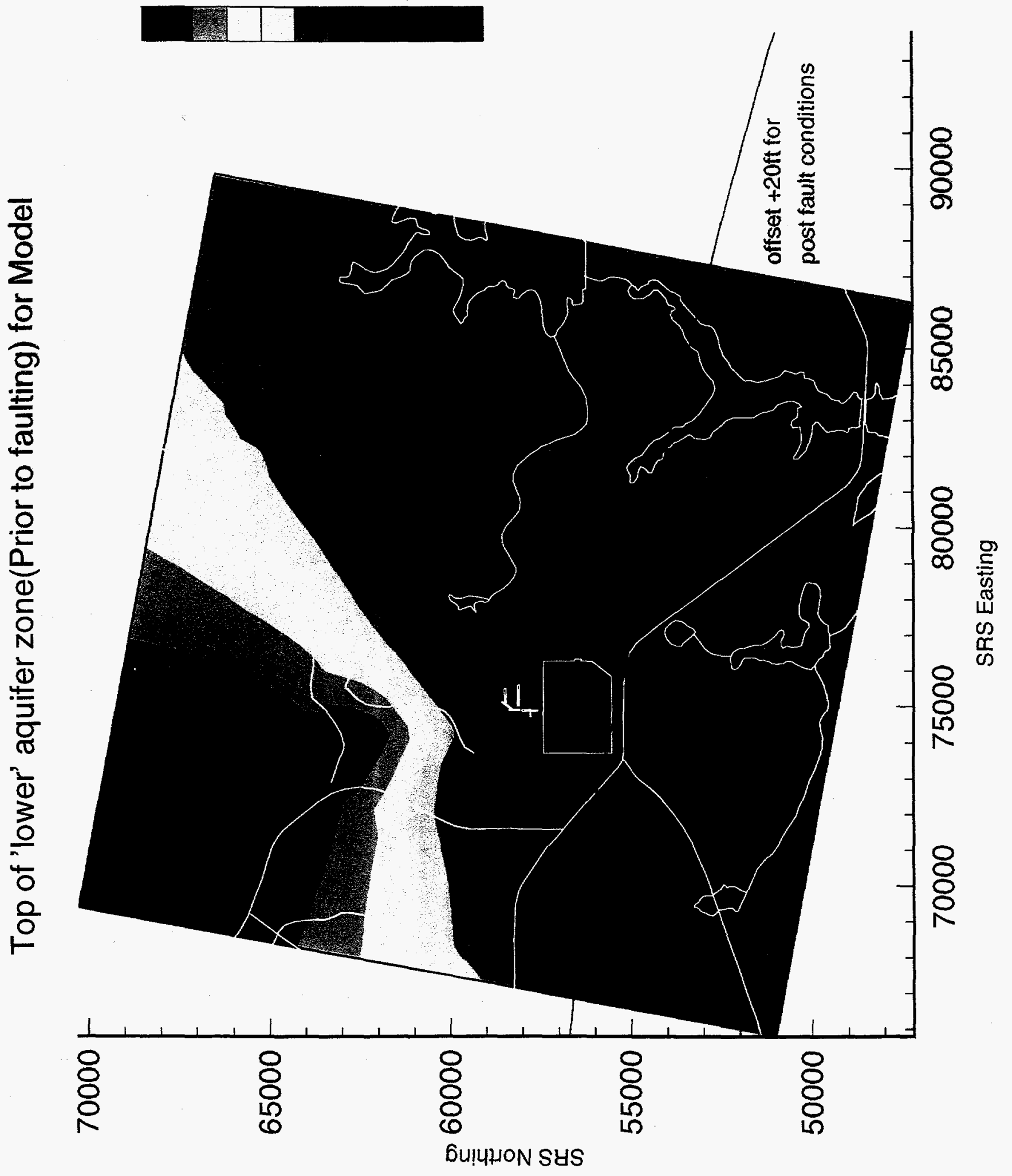




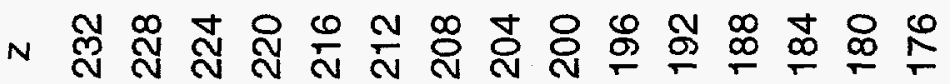

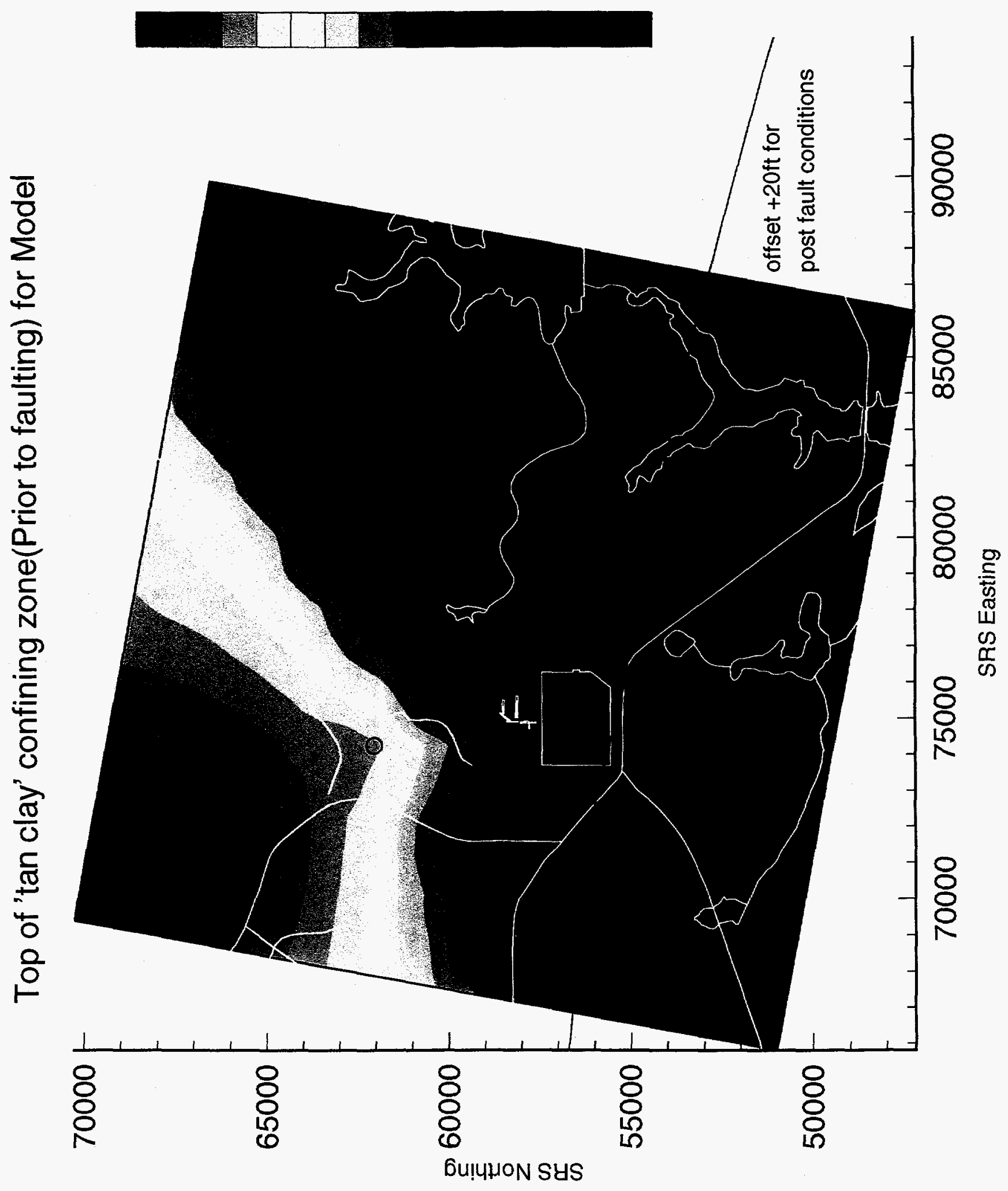


$N$ 品 员 N

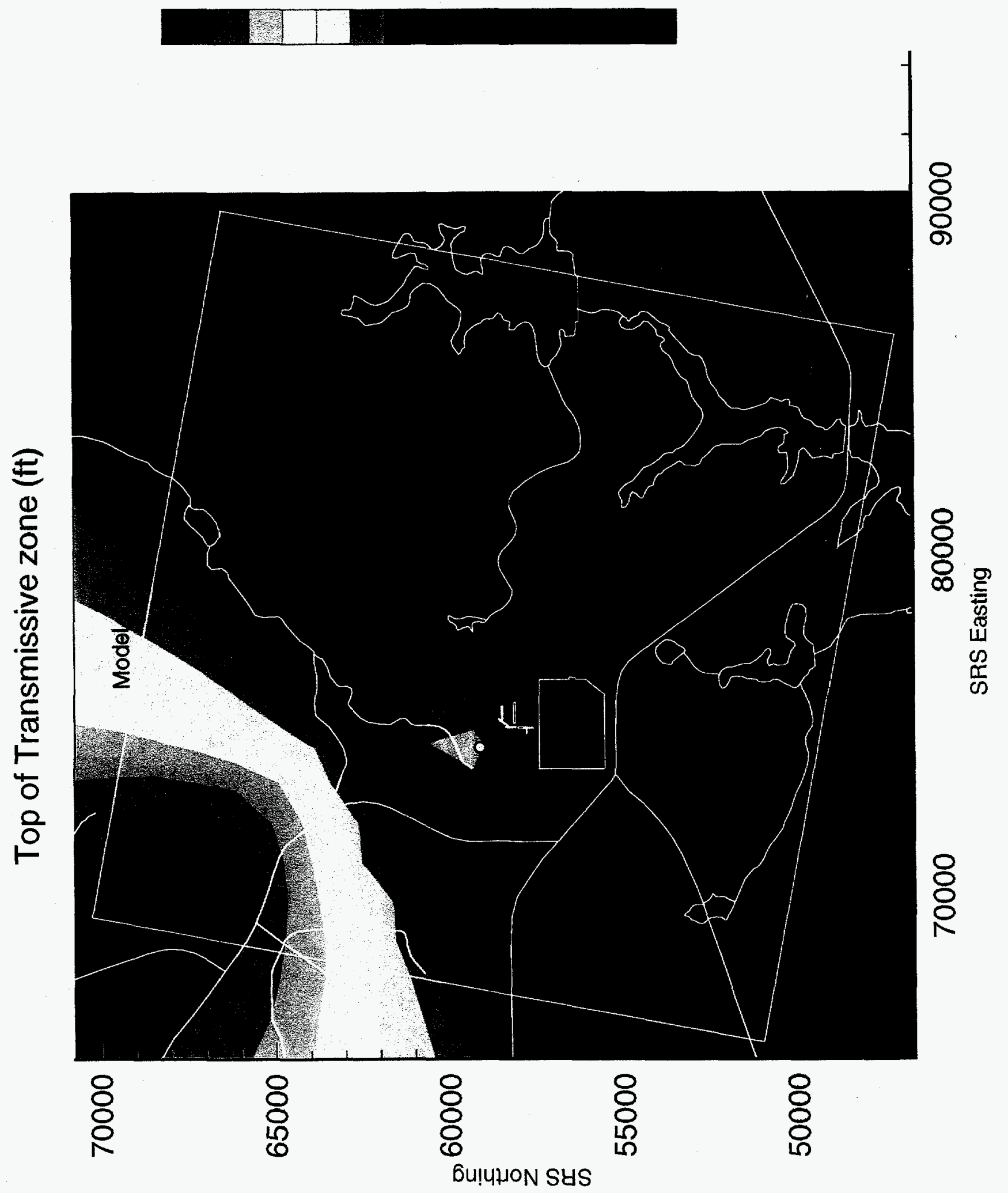

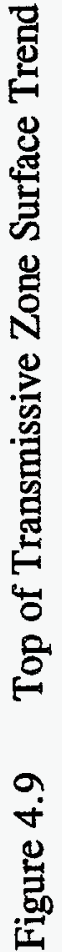


$N$ 负

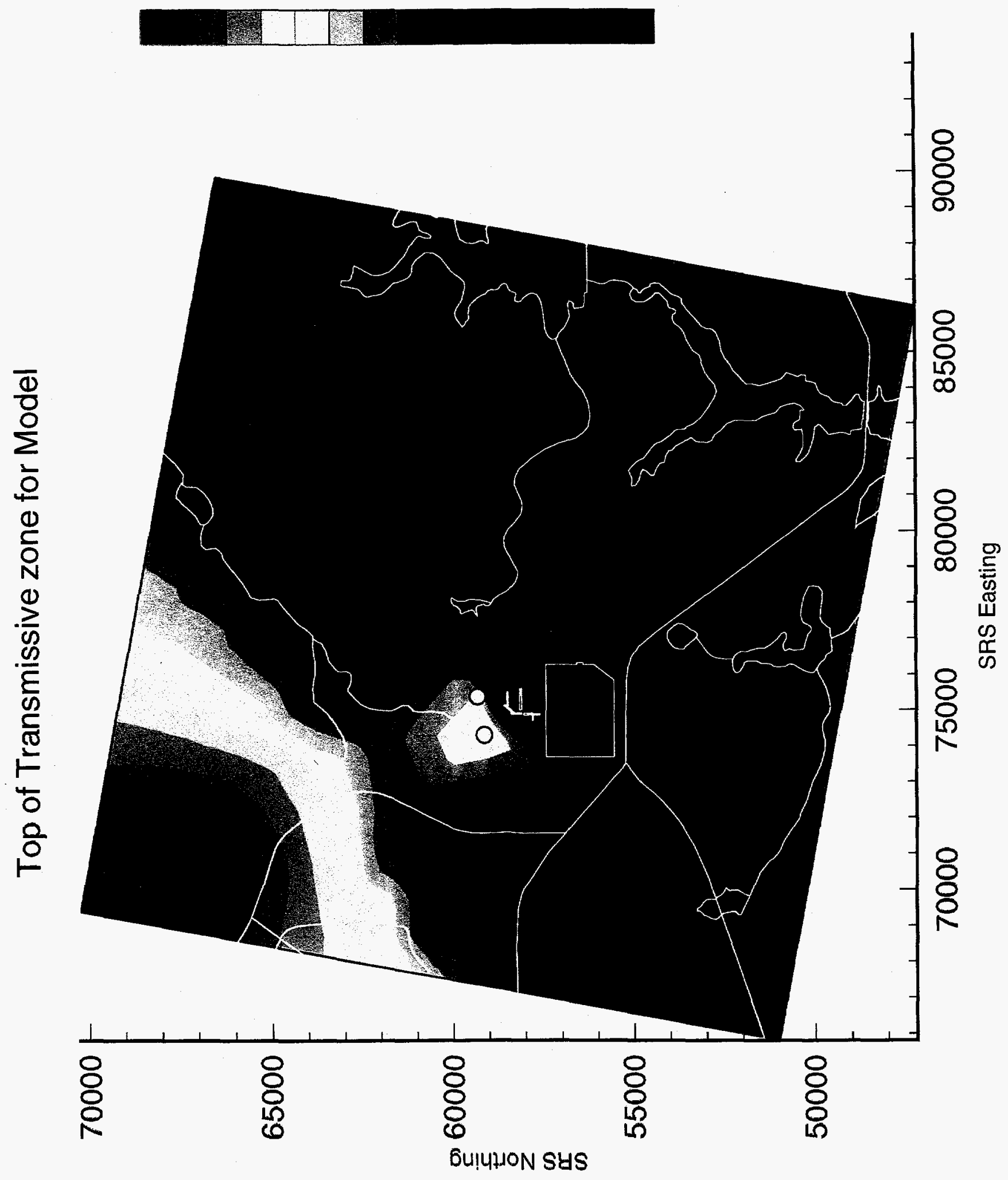

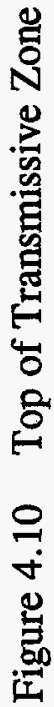




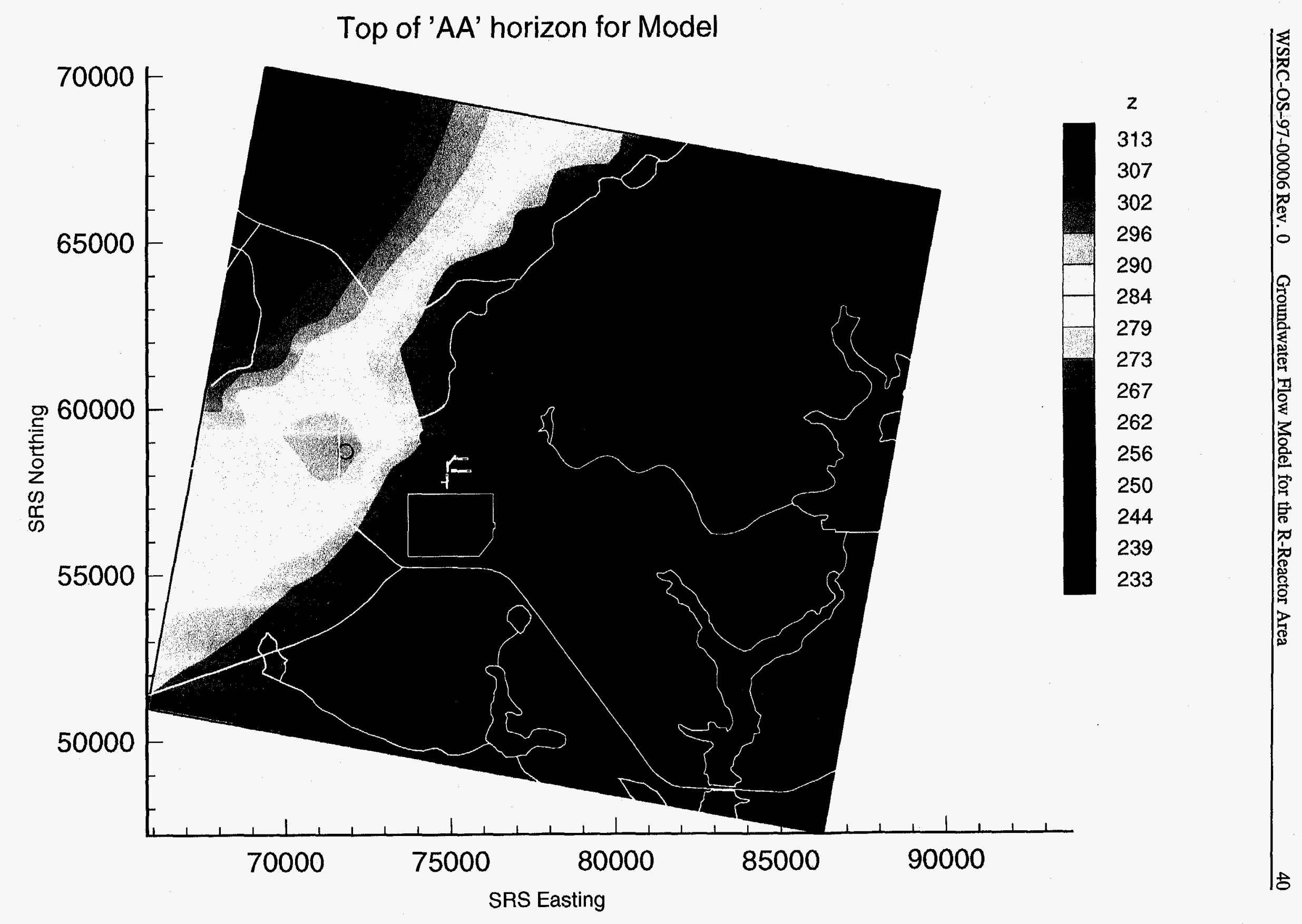

Figure 4.11 Top of "AA" Horizon 


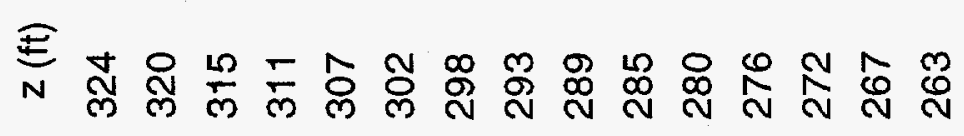

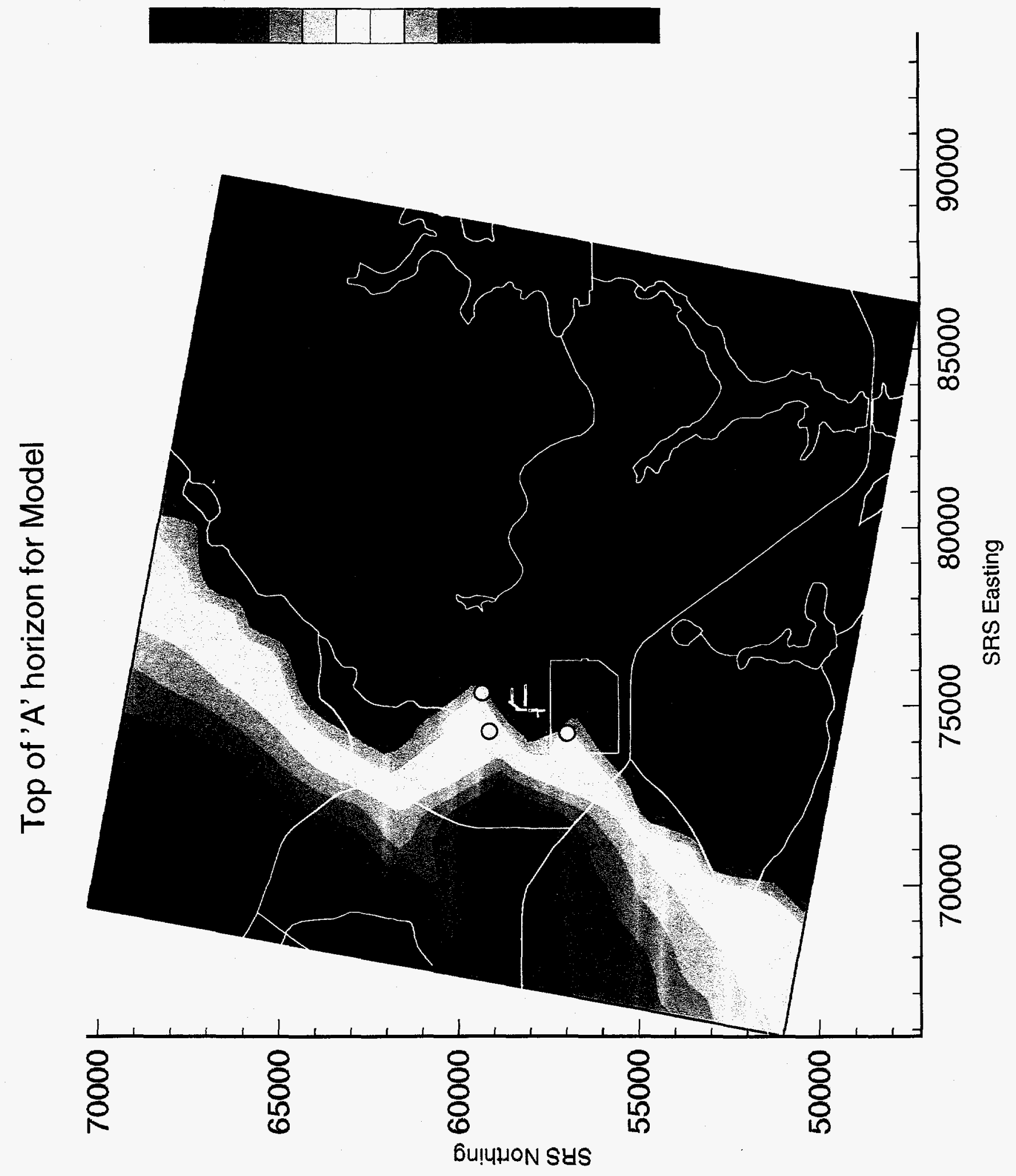

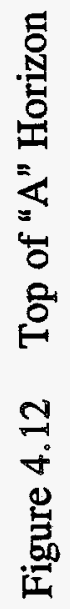



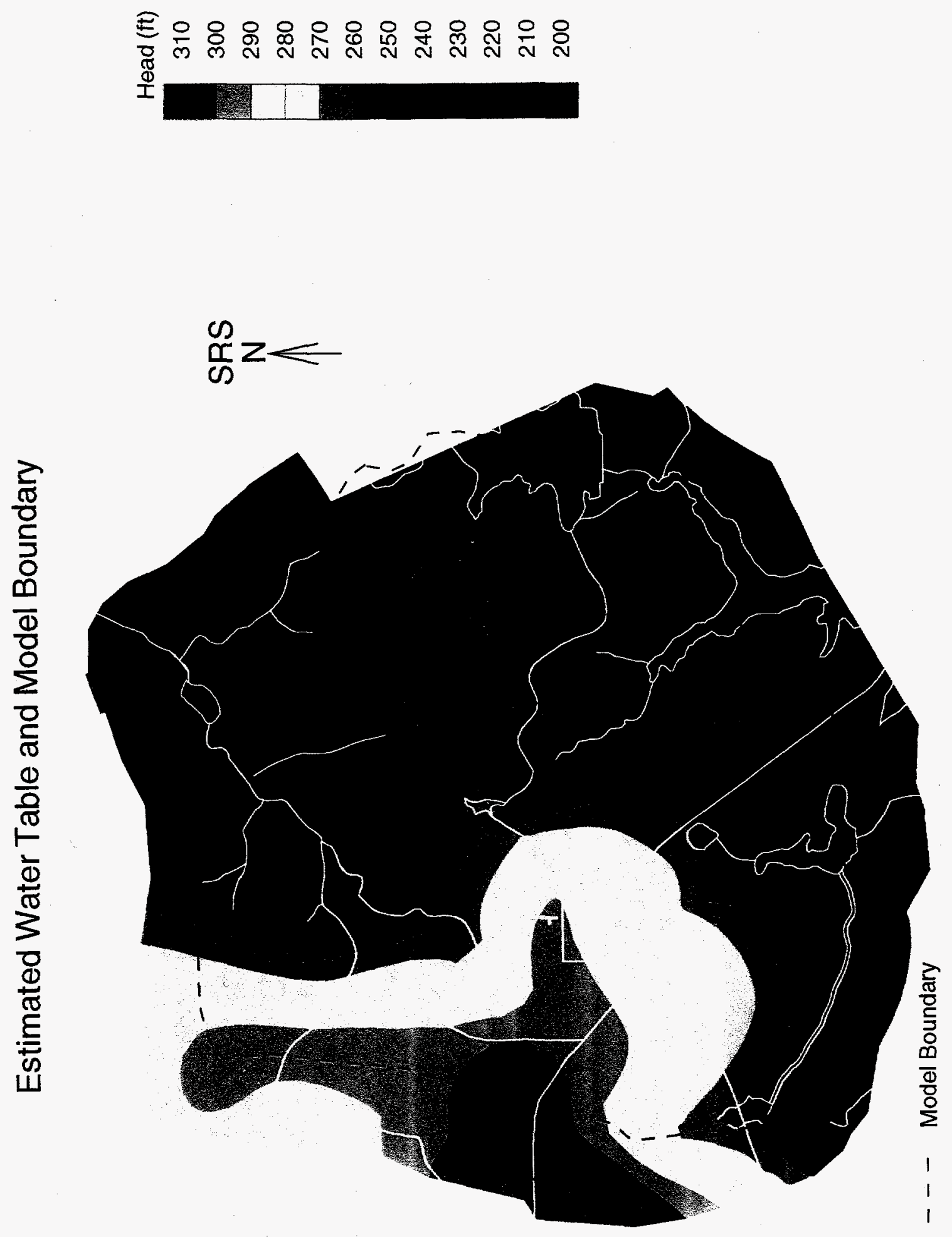

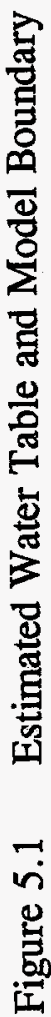




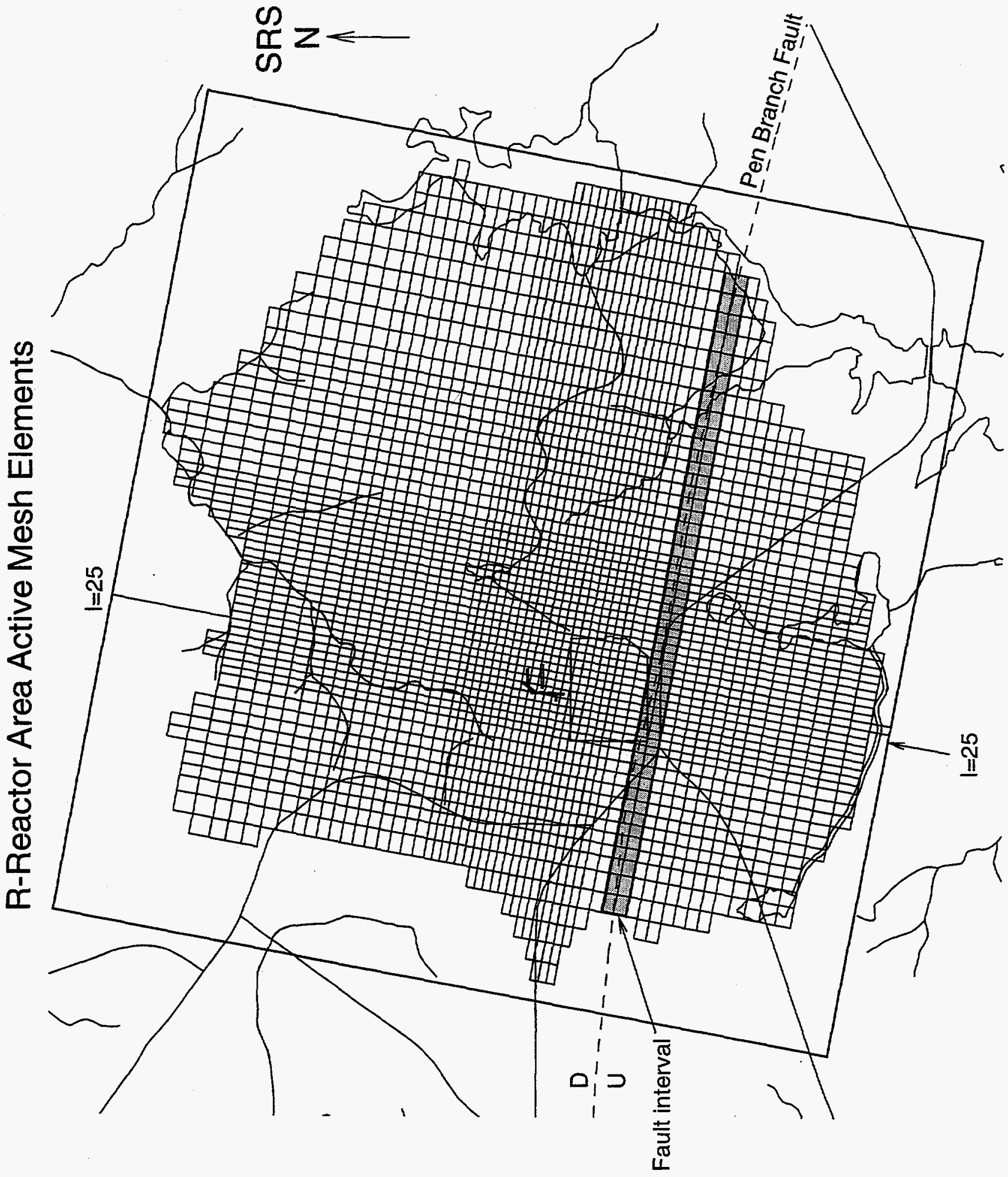

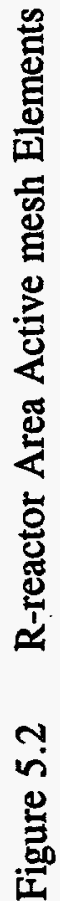



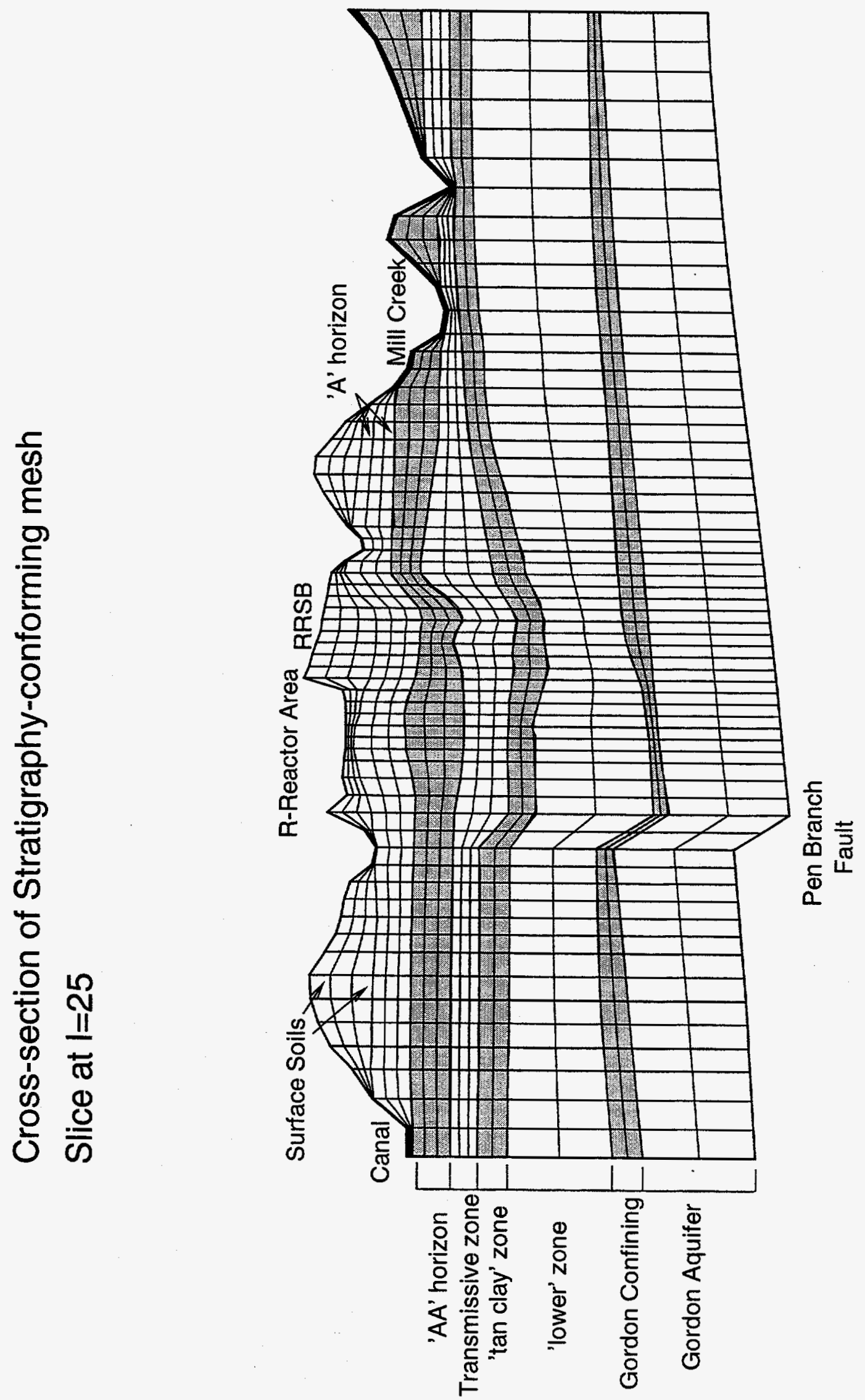


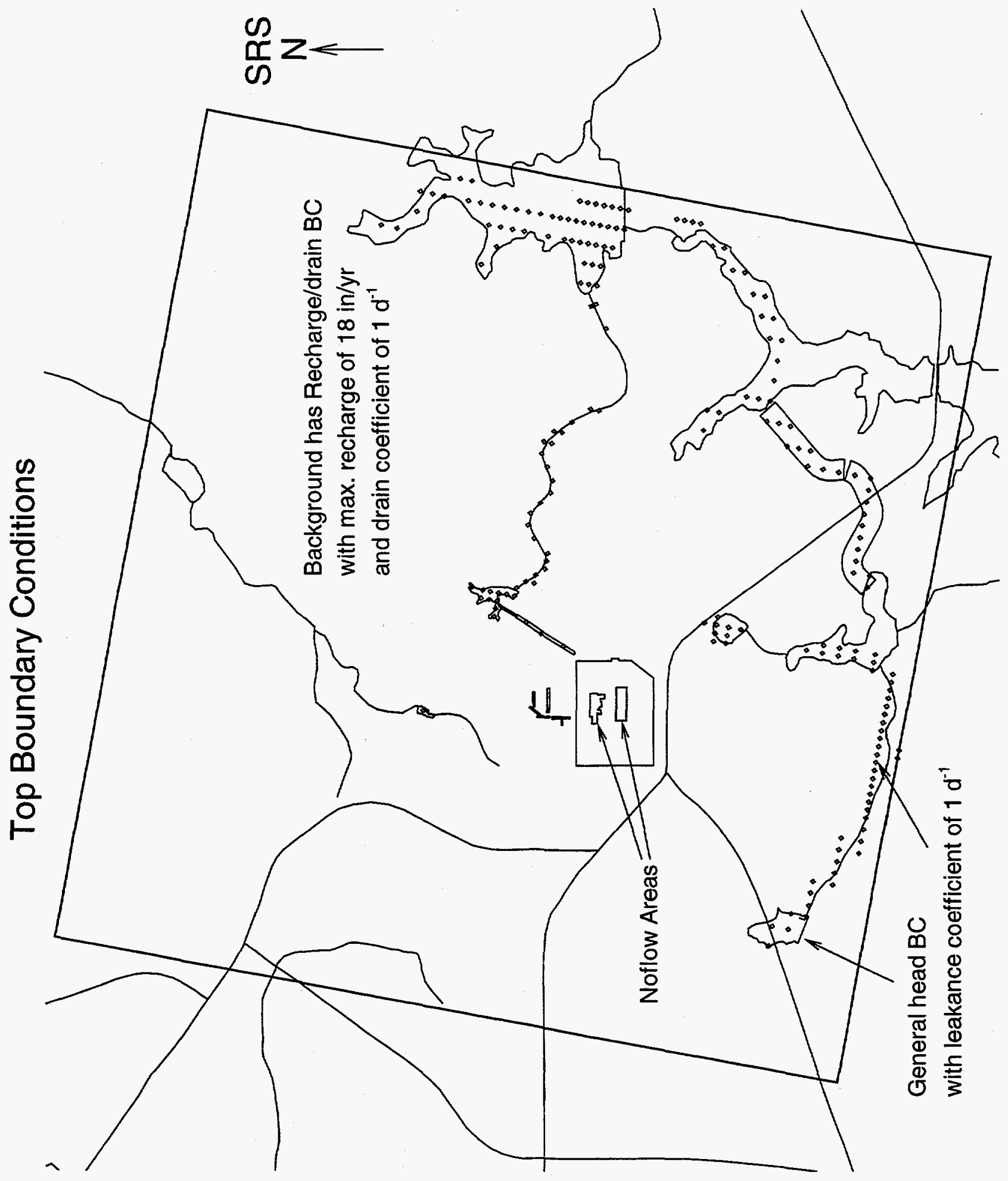

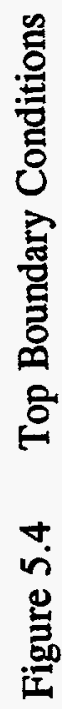




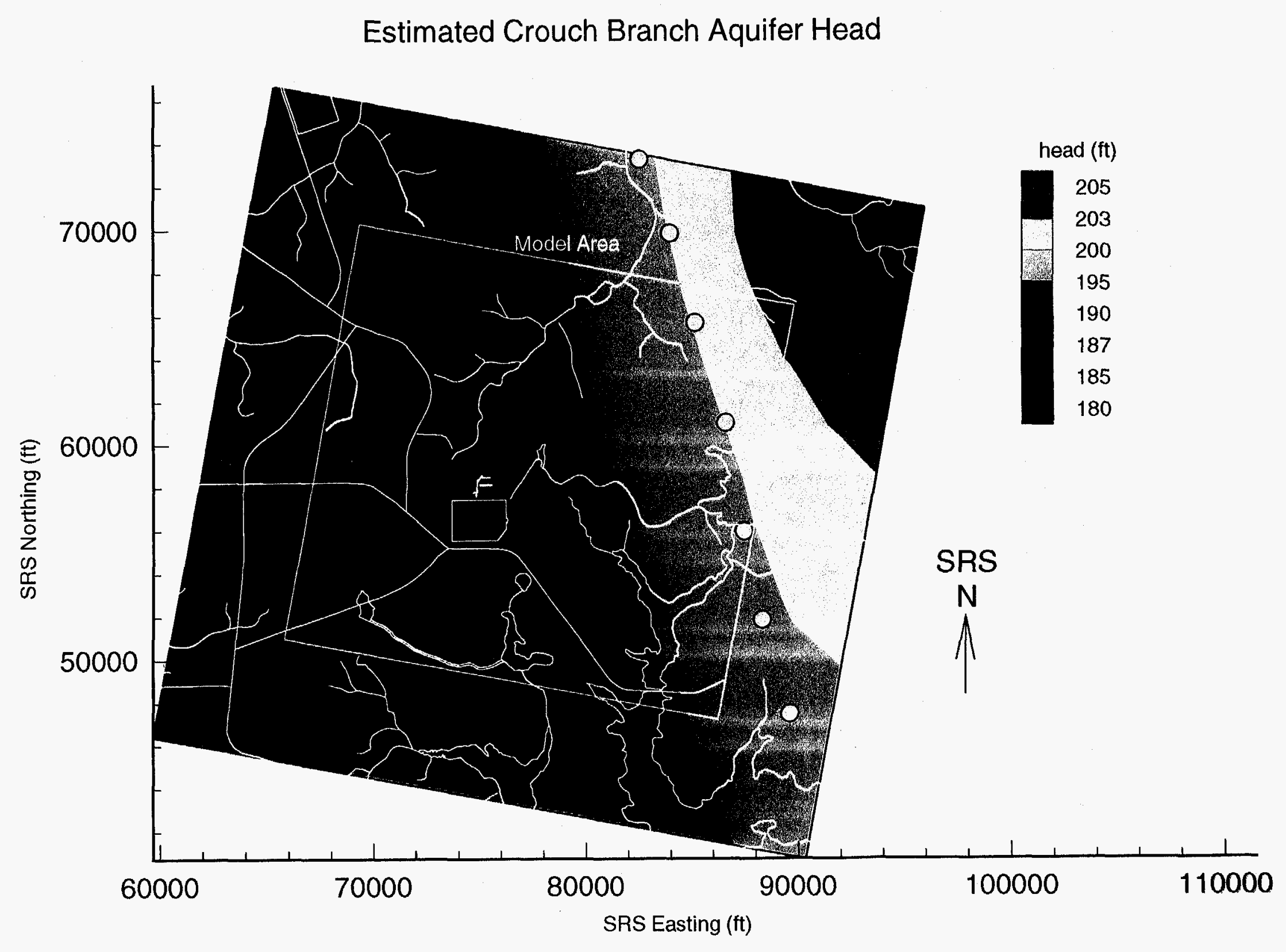

Figure 5.5 Estimated Crouch Branch Aquifer Head 


\section{R-Reactor Area Vertical Boundary Conditions}

Gordon: prescribed head lower: prescribed head upper: noflow

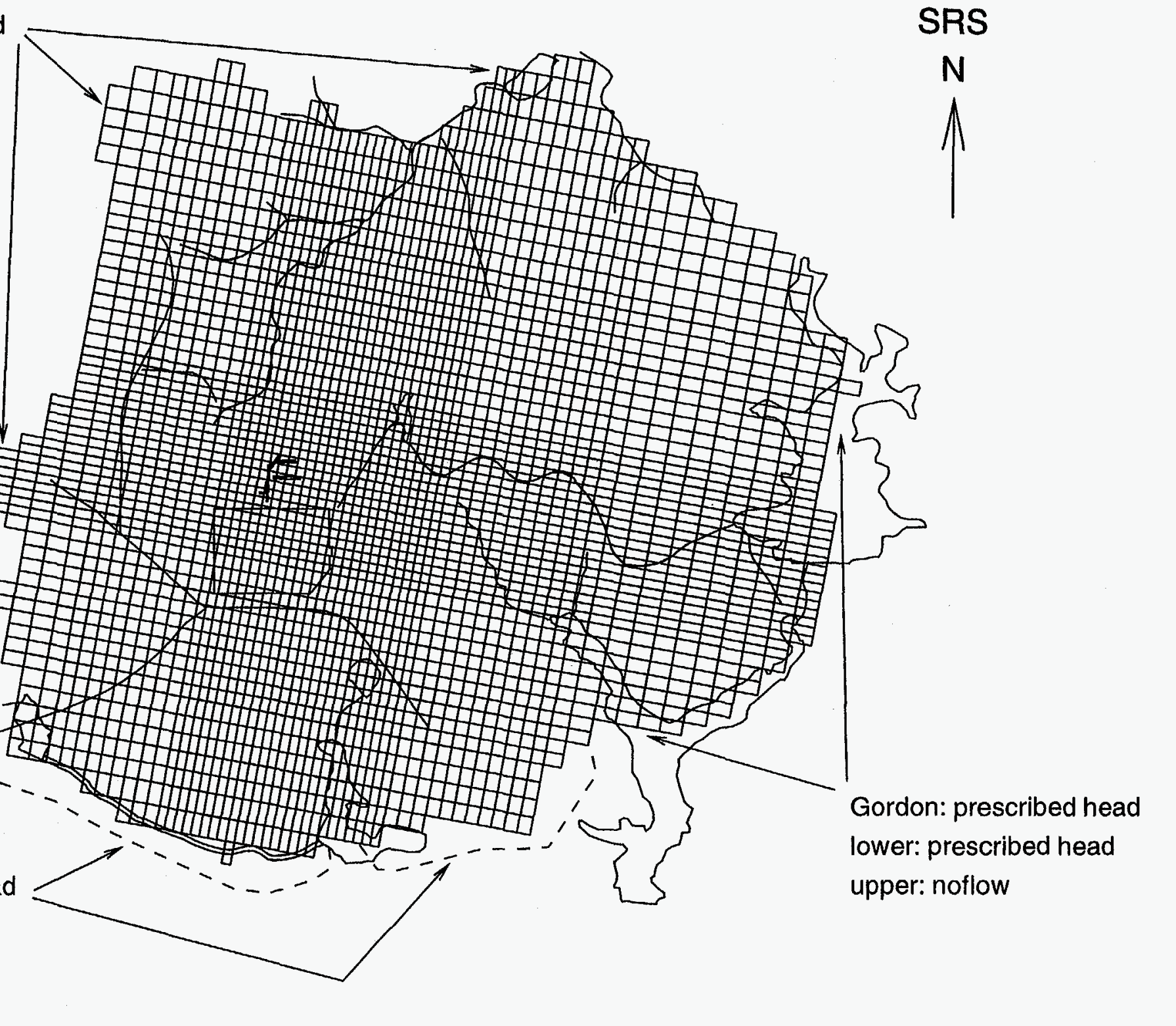

Figure 5.6 Vertical Boundary Conditions 


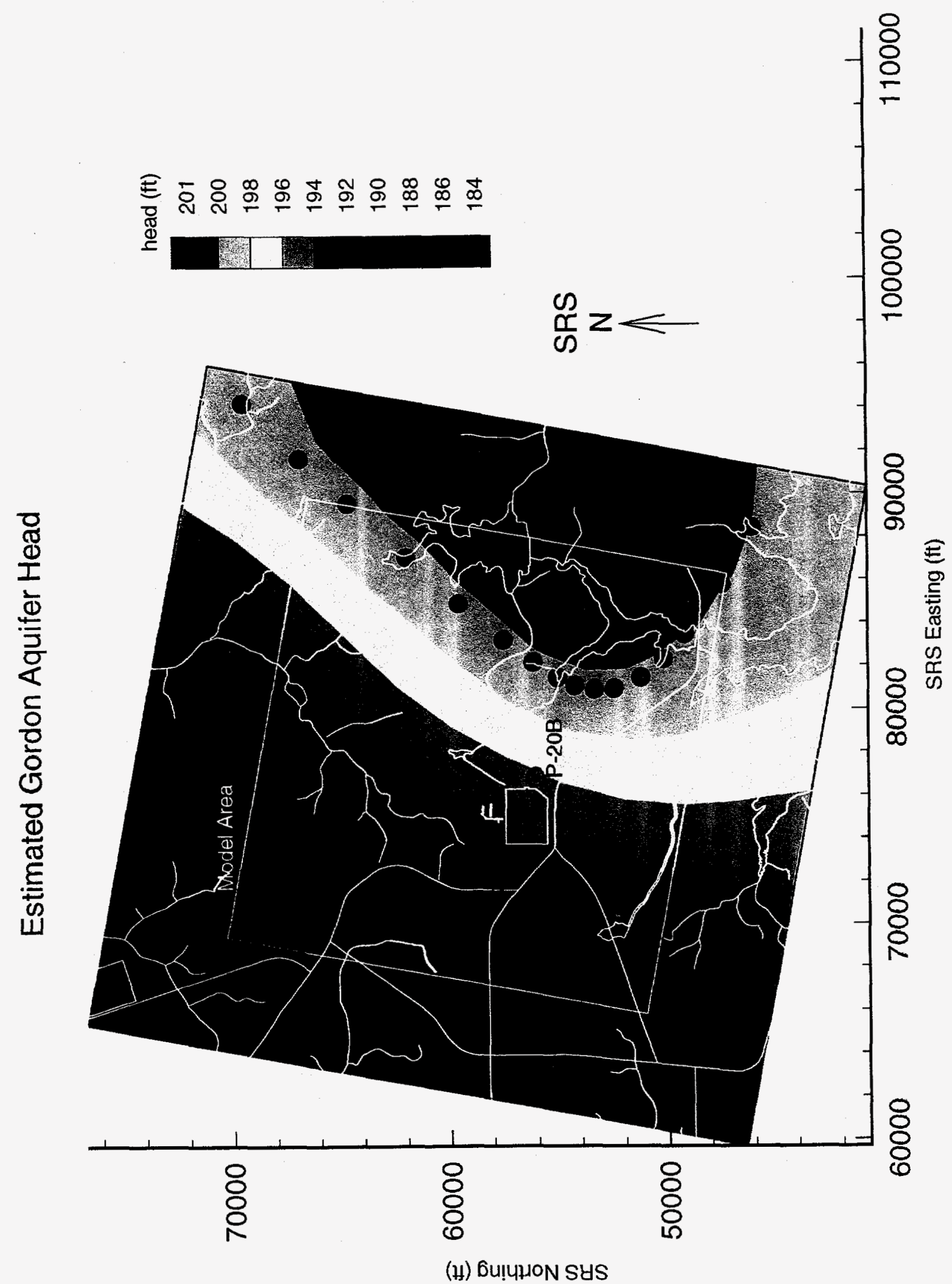

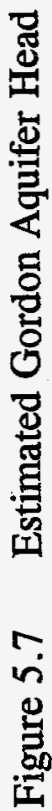




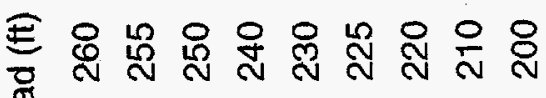

ॠ

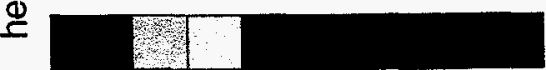

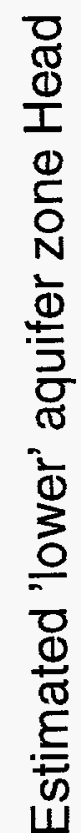

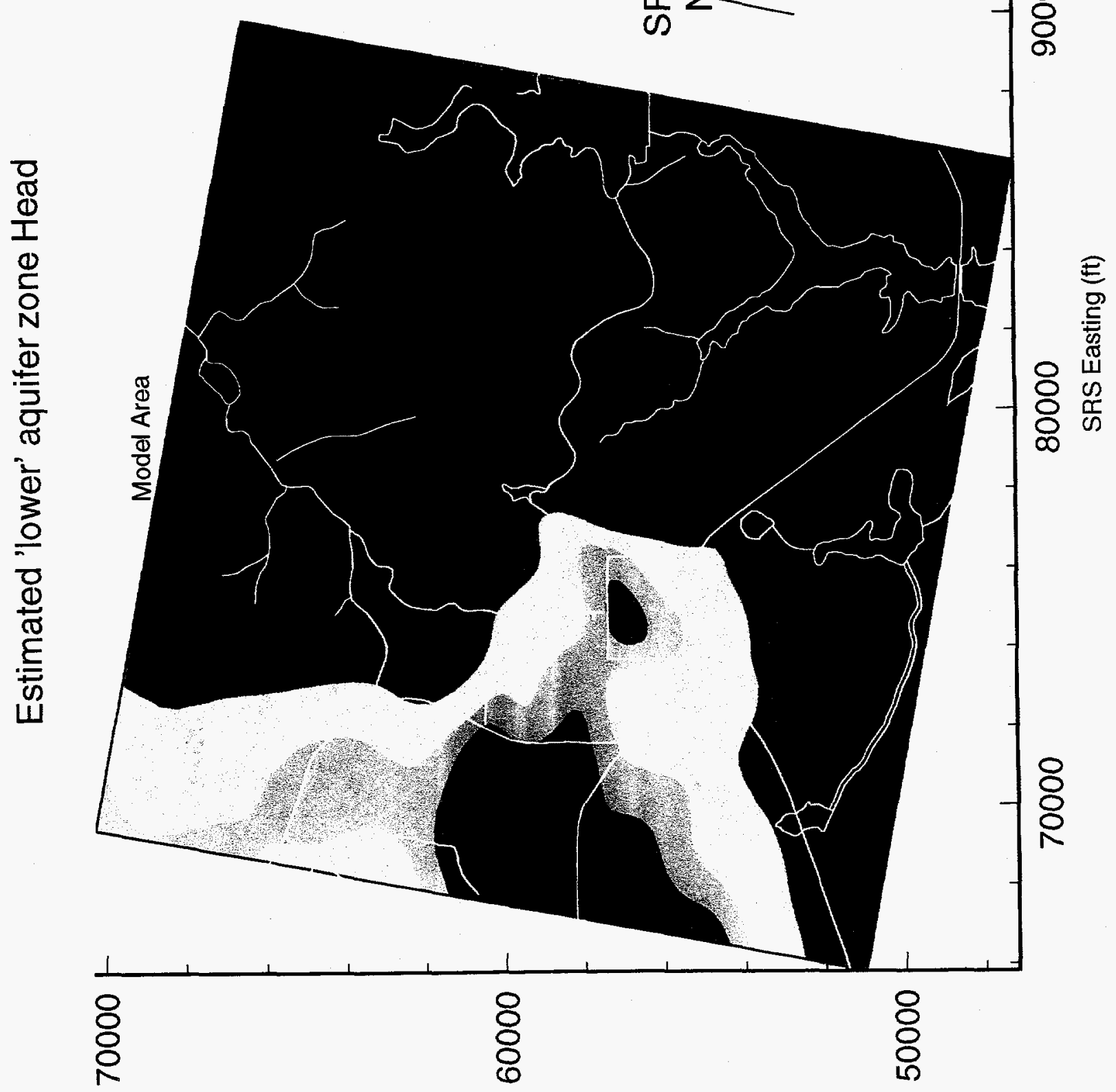

(H) 6u!YHON SUS 


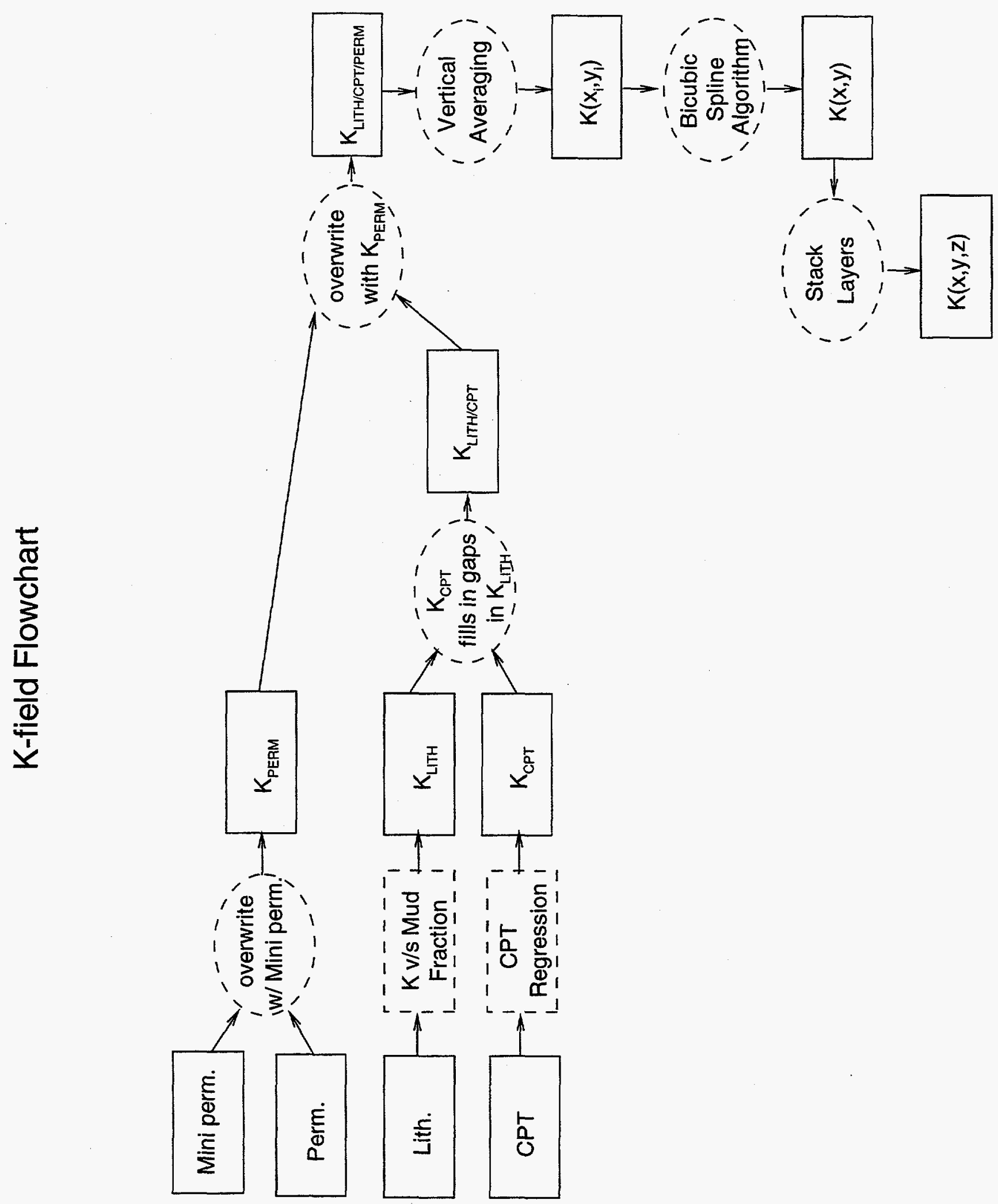




\section{$\mathrm{K}_{\mathrm{h}}$ Distribution for Gordon Aquifer}

\section{Element layer 1}
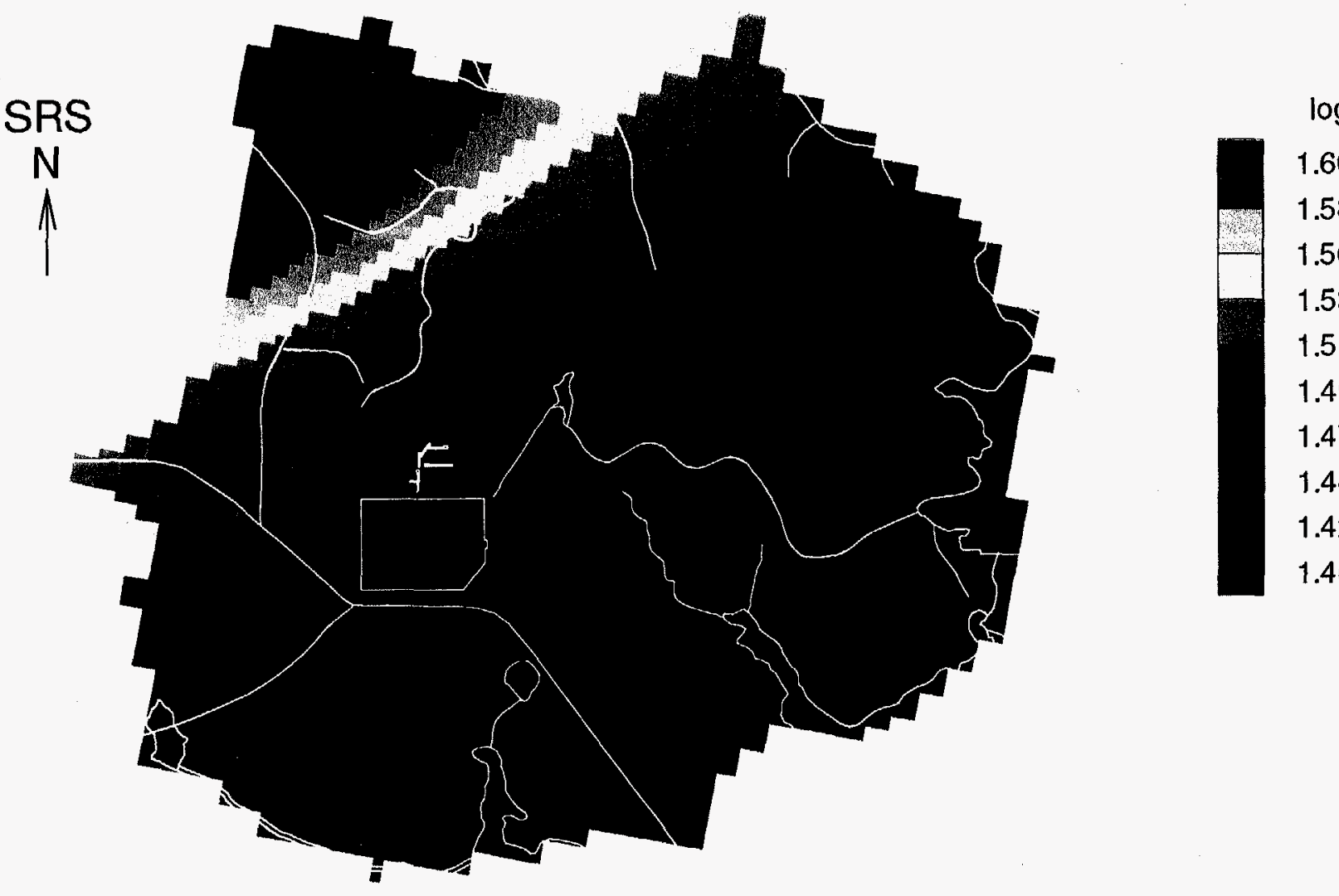

$\log 10(\mathrm{Kh})$

1.60

1.58

1.56

1.53

1.51

1.49

1.47

1.44

1.42

1.40

Figure 5.10 $\quad \mathrm{K}_{\mathrm{h}}$ Distribution for Gordon Aquifer, Element Layer 1 


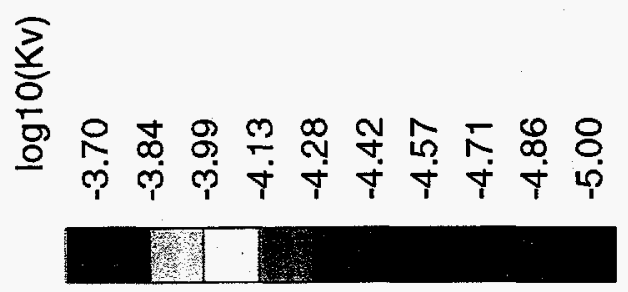

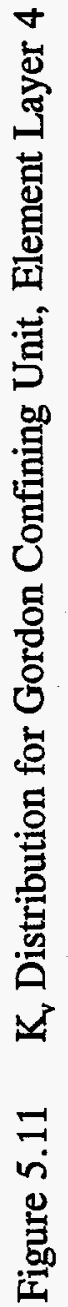

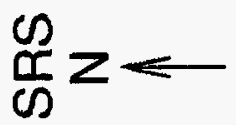



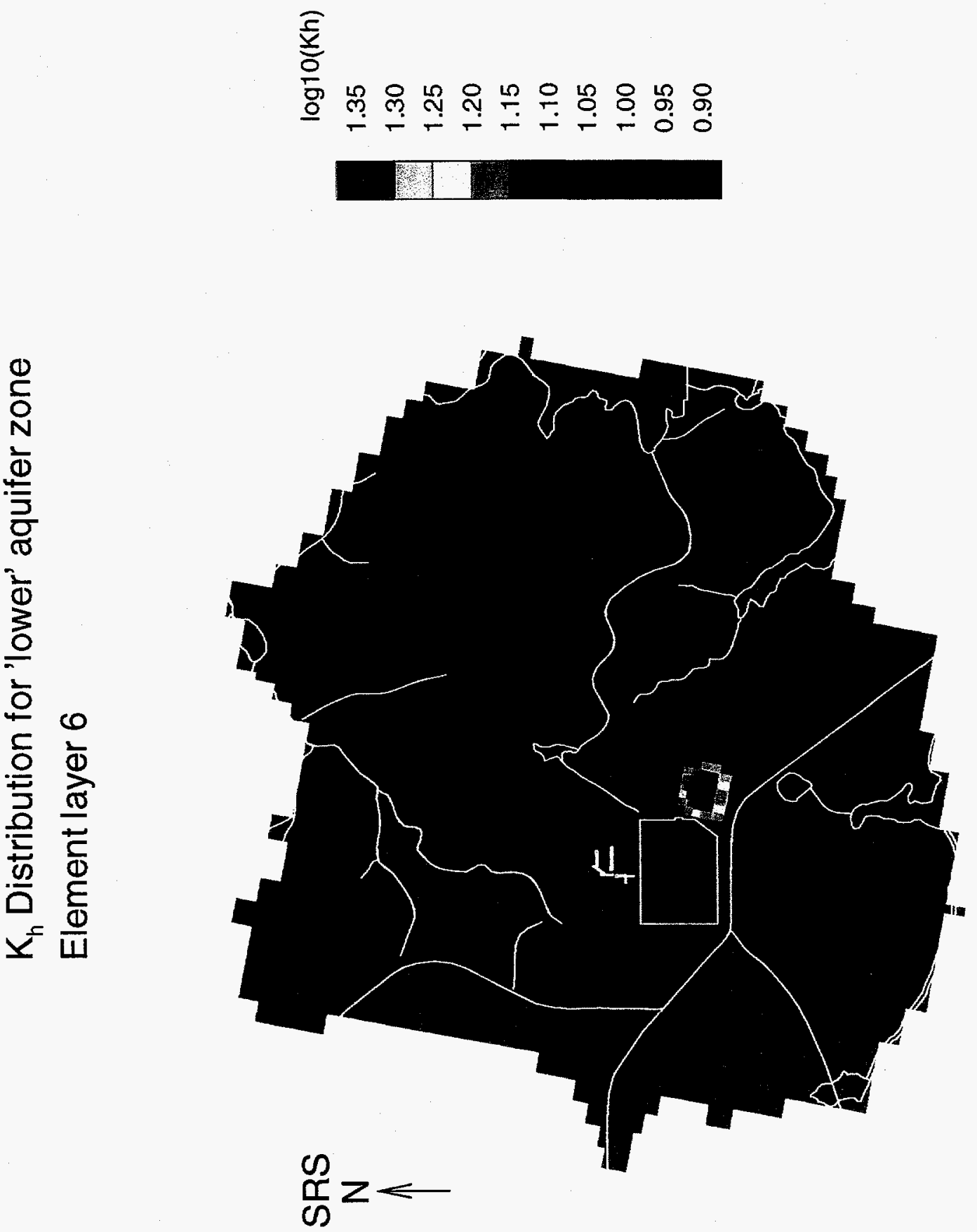
$\mathrm{K}_{v}$ Distribution for 'tan clay' confining zone

\section{Element layer 7}

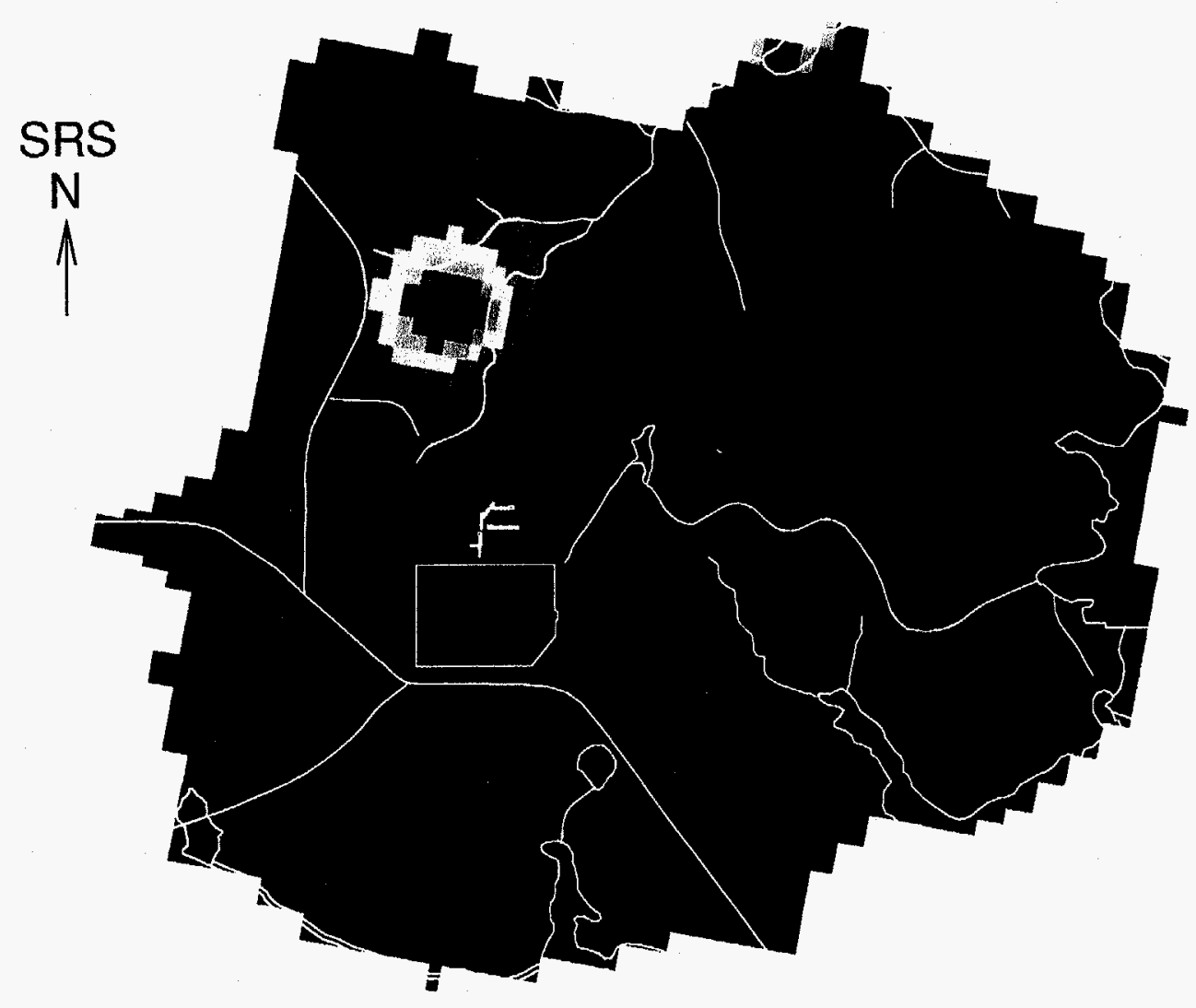

Figure 5.13 $\mathrm{K}_{\mathrm{v}}$ Distribution for "tan clay" Confining Zone, Element Layer 7 

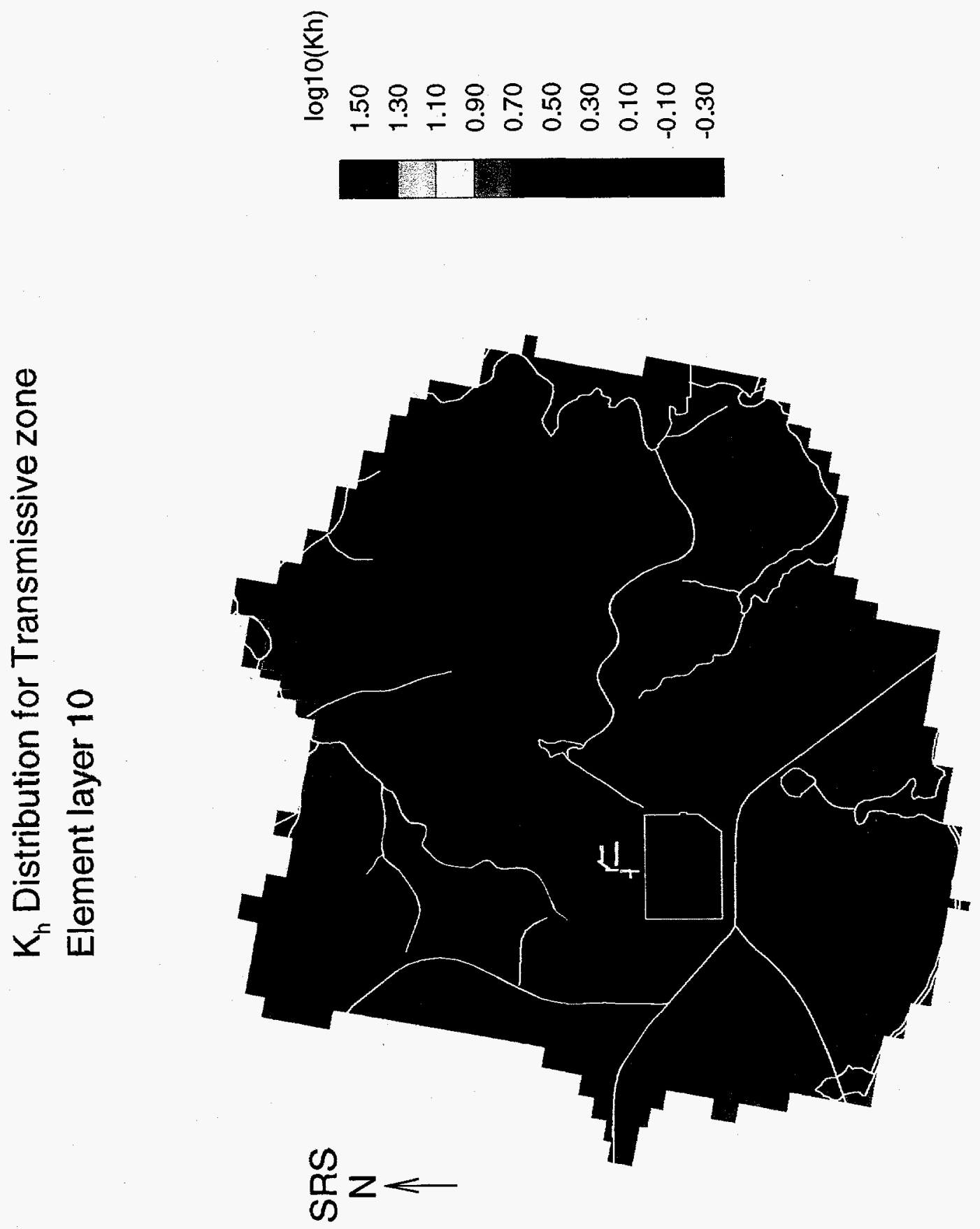

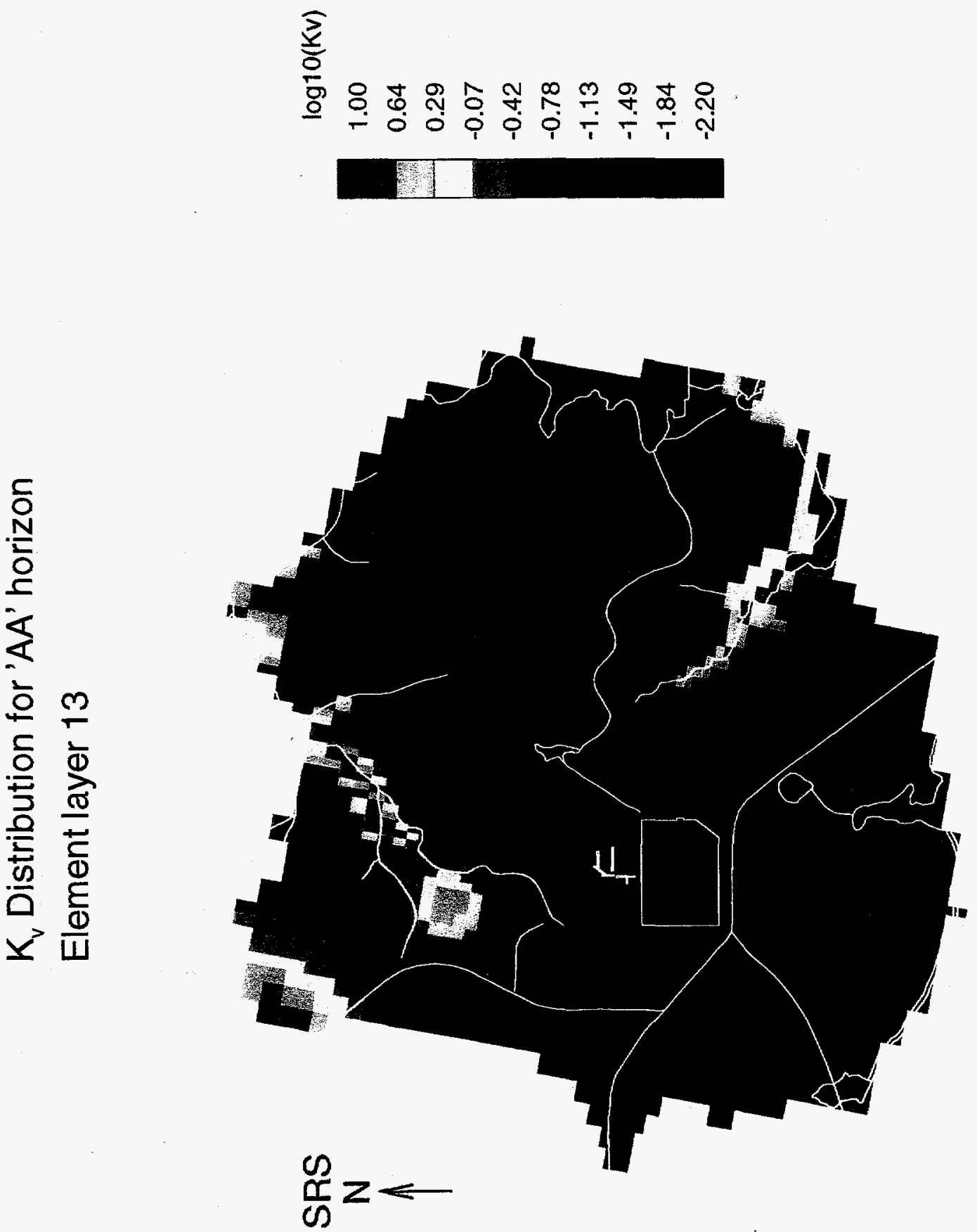

굴 

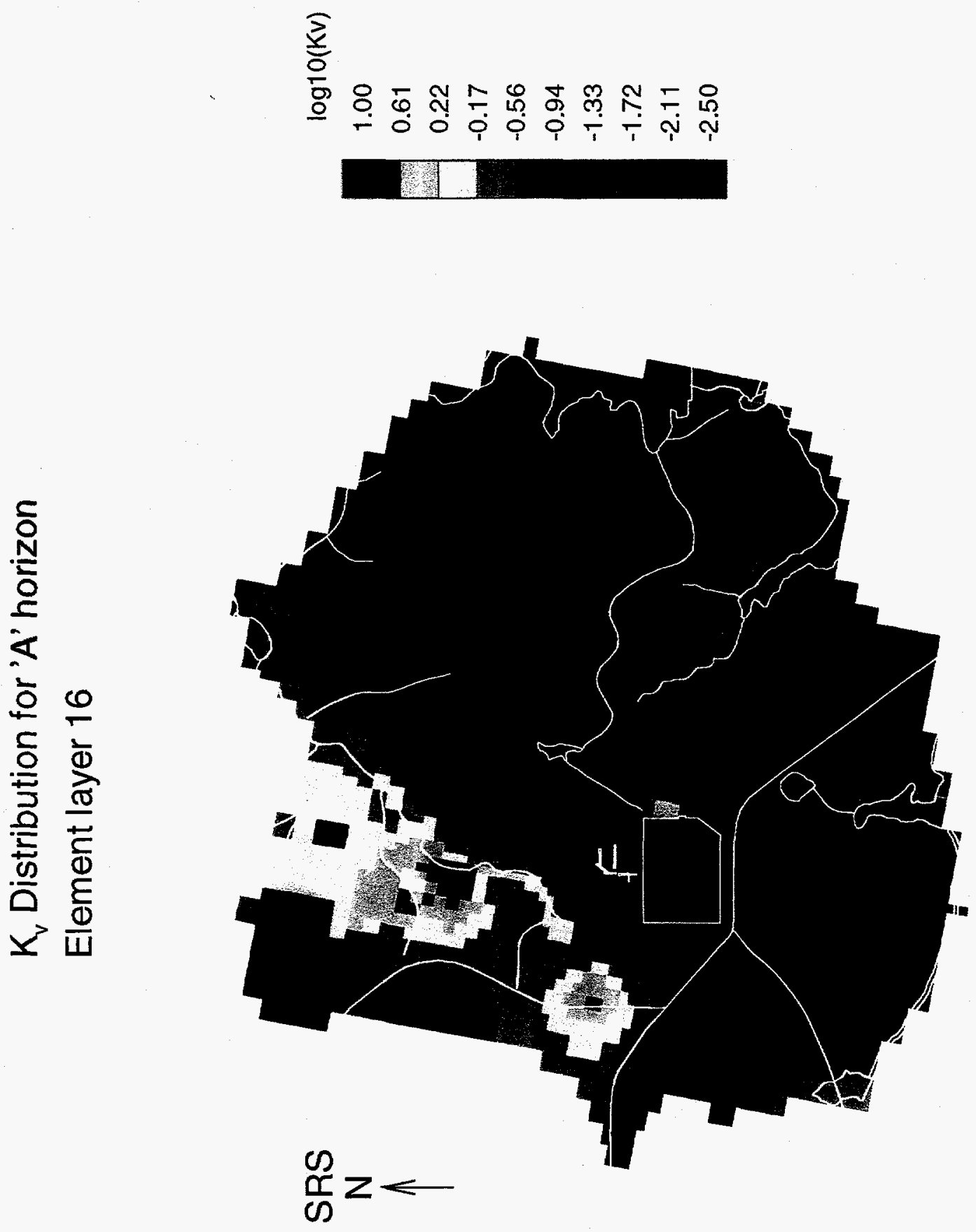

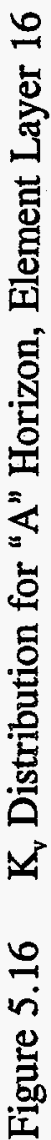



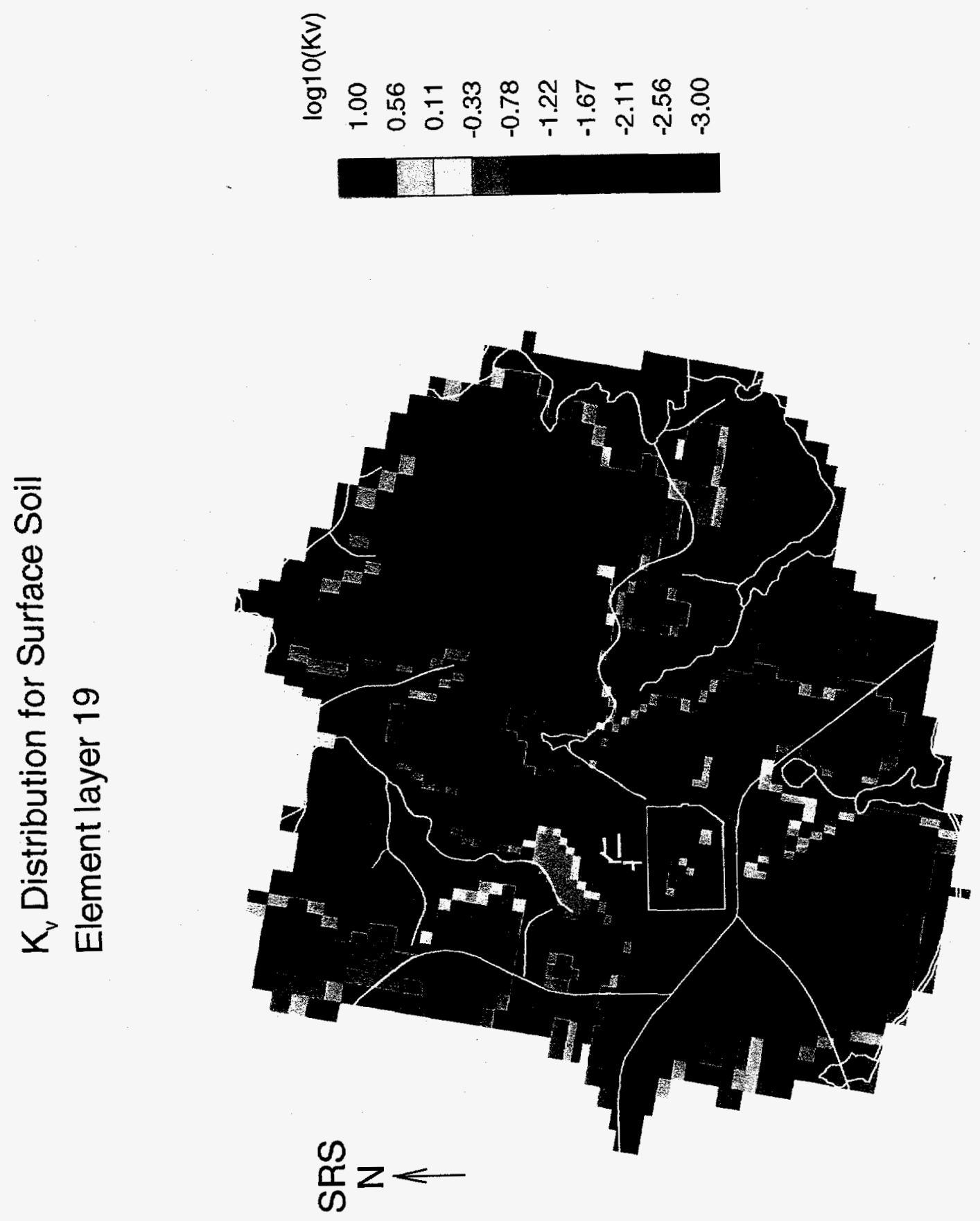


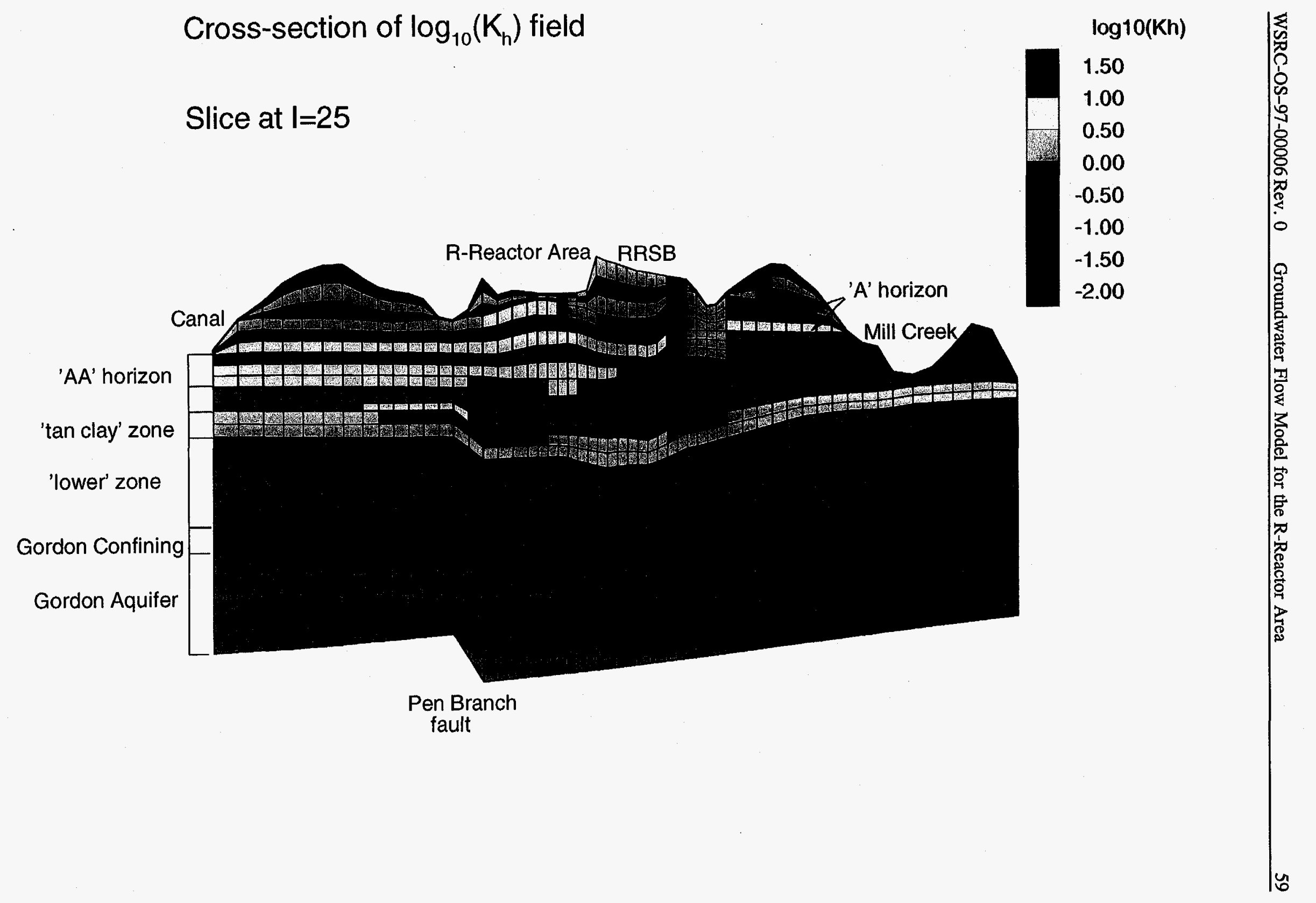

Figure 5.18 $\quad \mathrm{K}_{\mathrm{h}}$ Distribution at $\mathrm{I}=25$ 


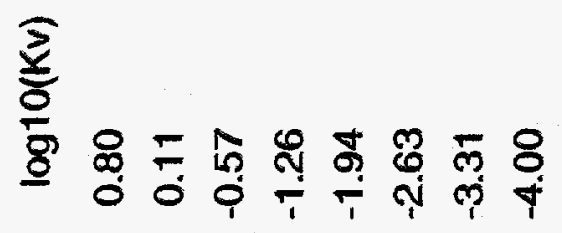
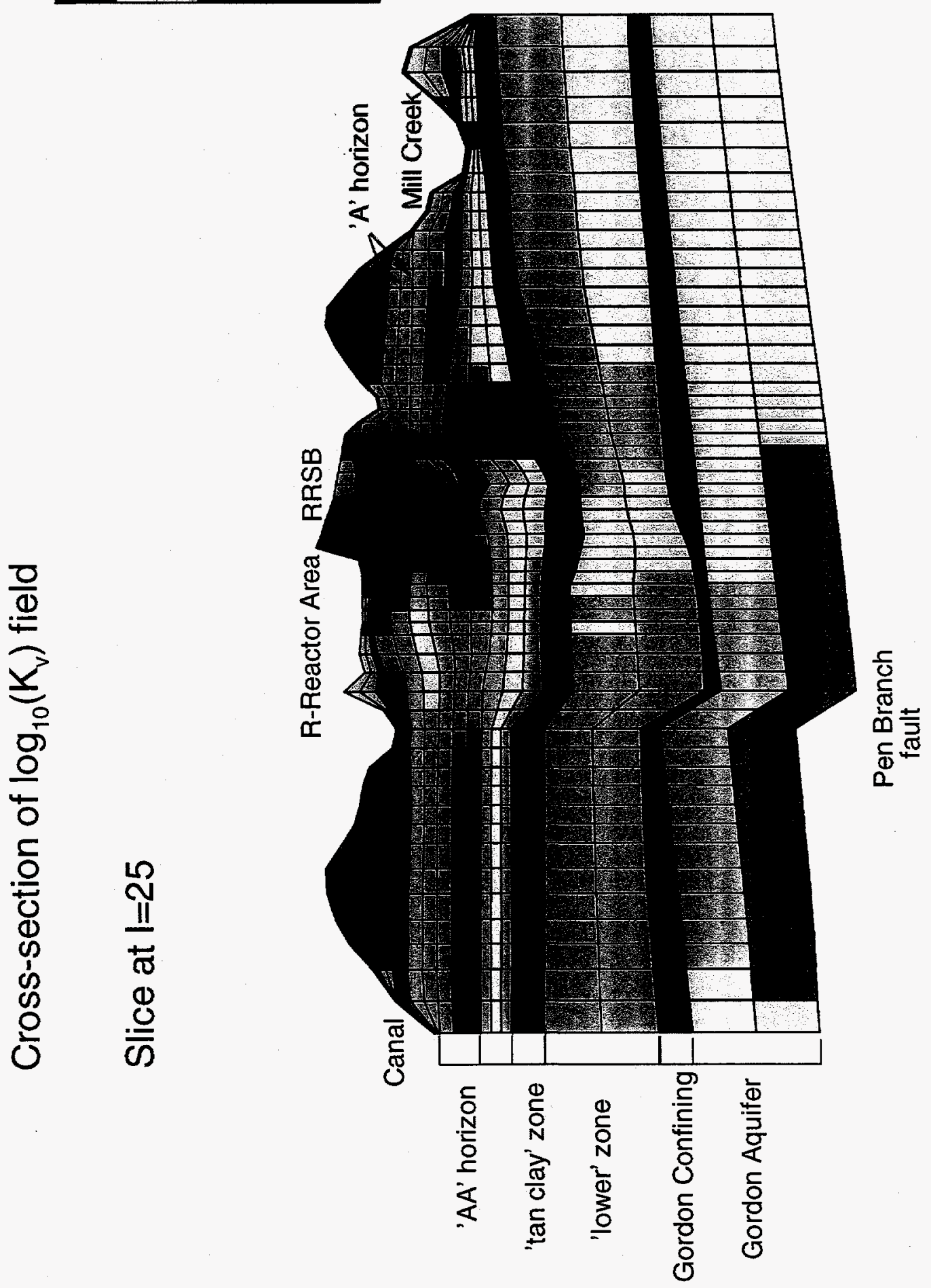

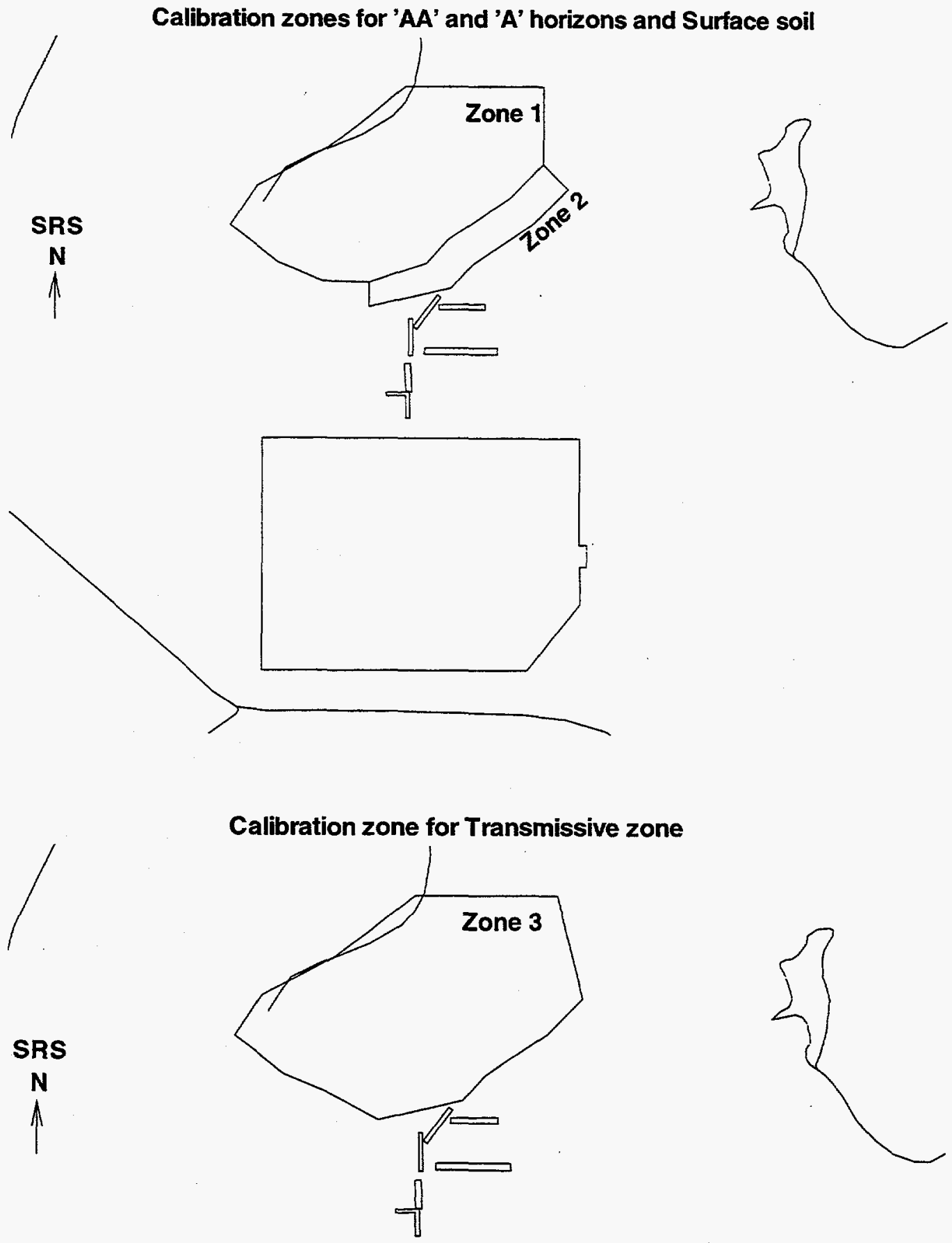

Calibration zone for Transmissive zone

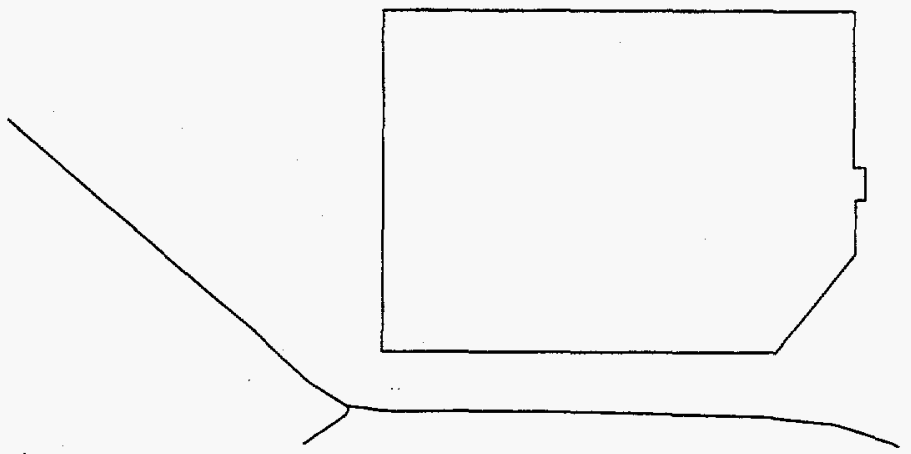

Figure 6.1 Calibration Zones 


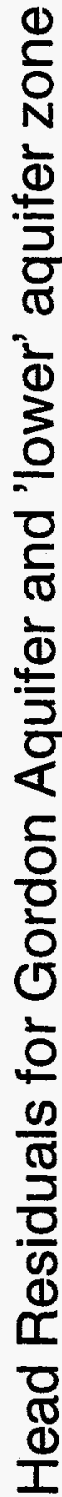

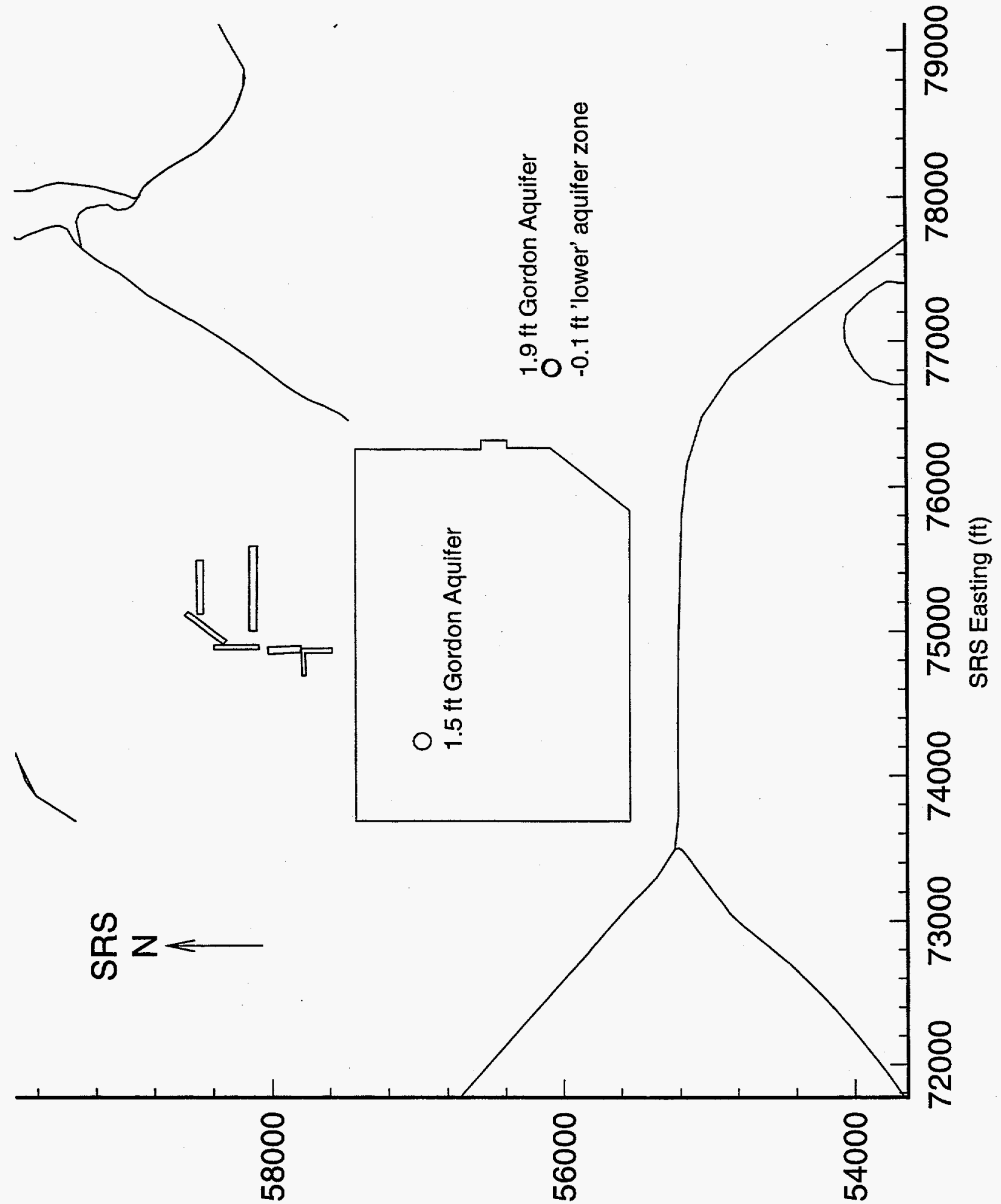

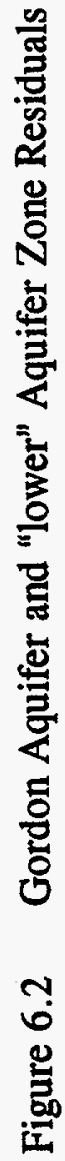

(H) bU!YHON SHS 


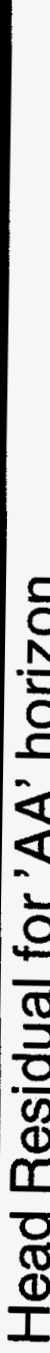

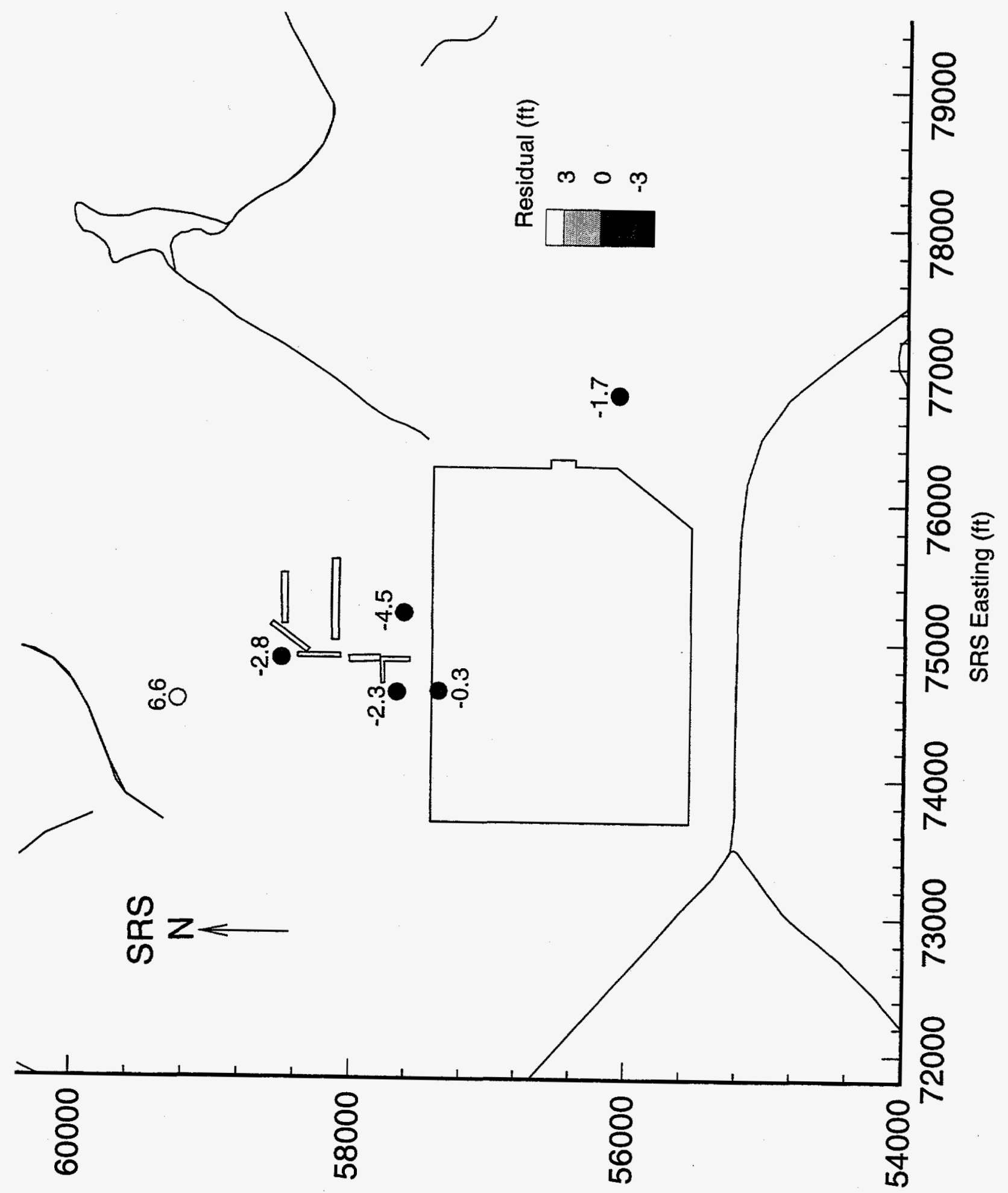

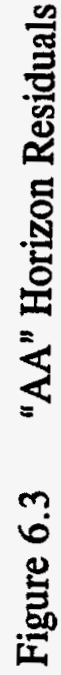

(H) 6u!บนON SUS 


\section{Head Residual for 'A' horizon}

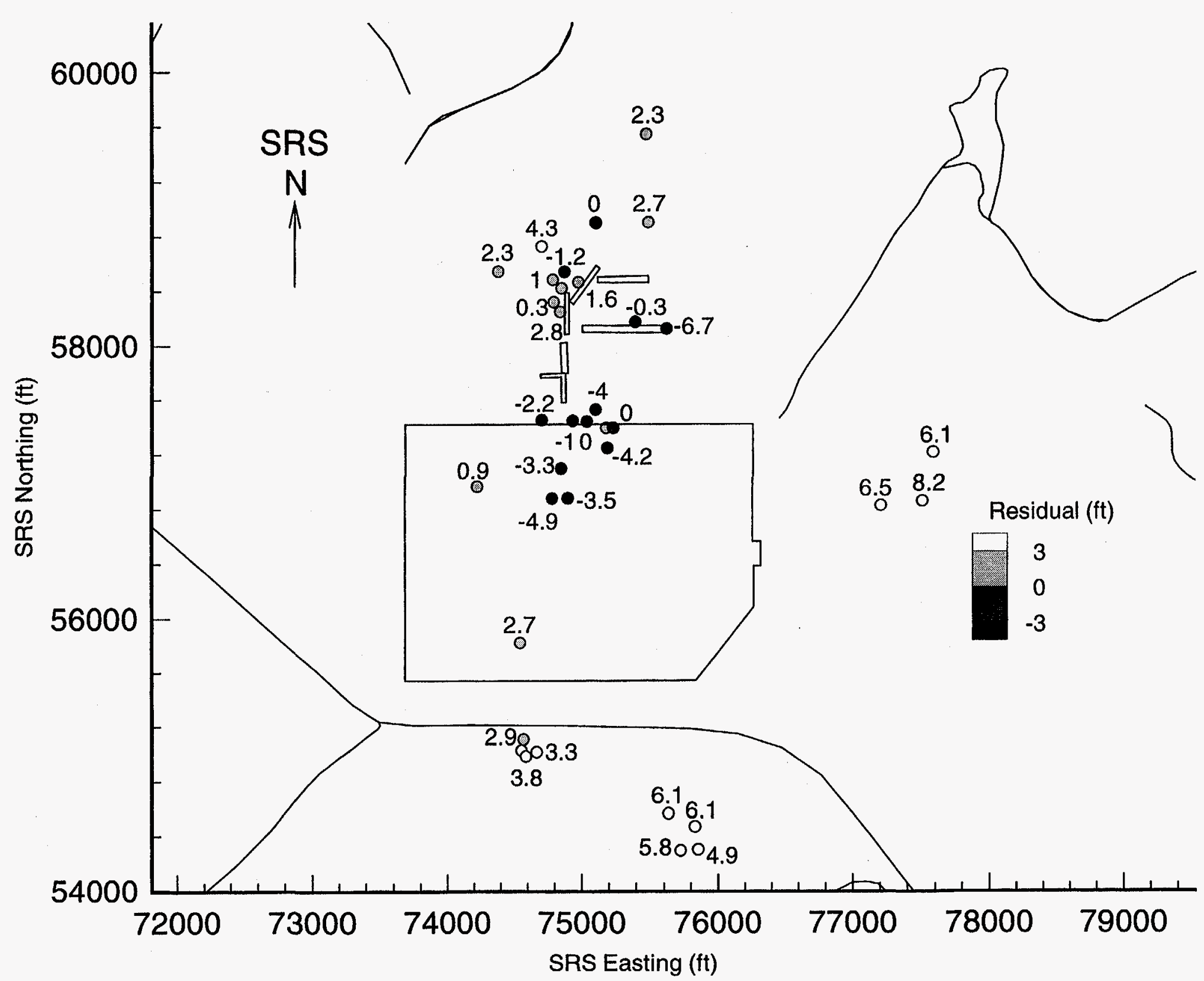




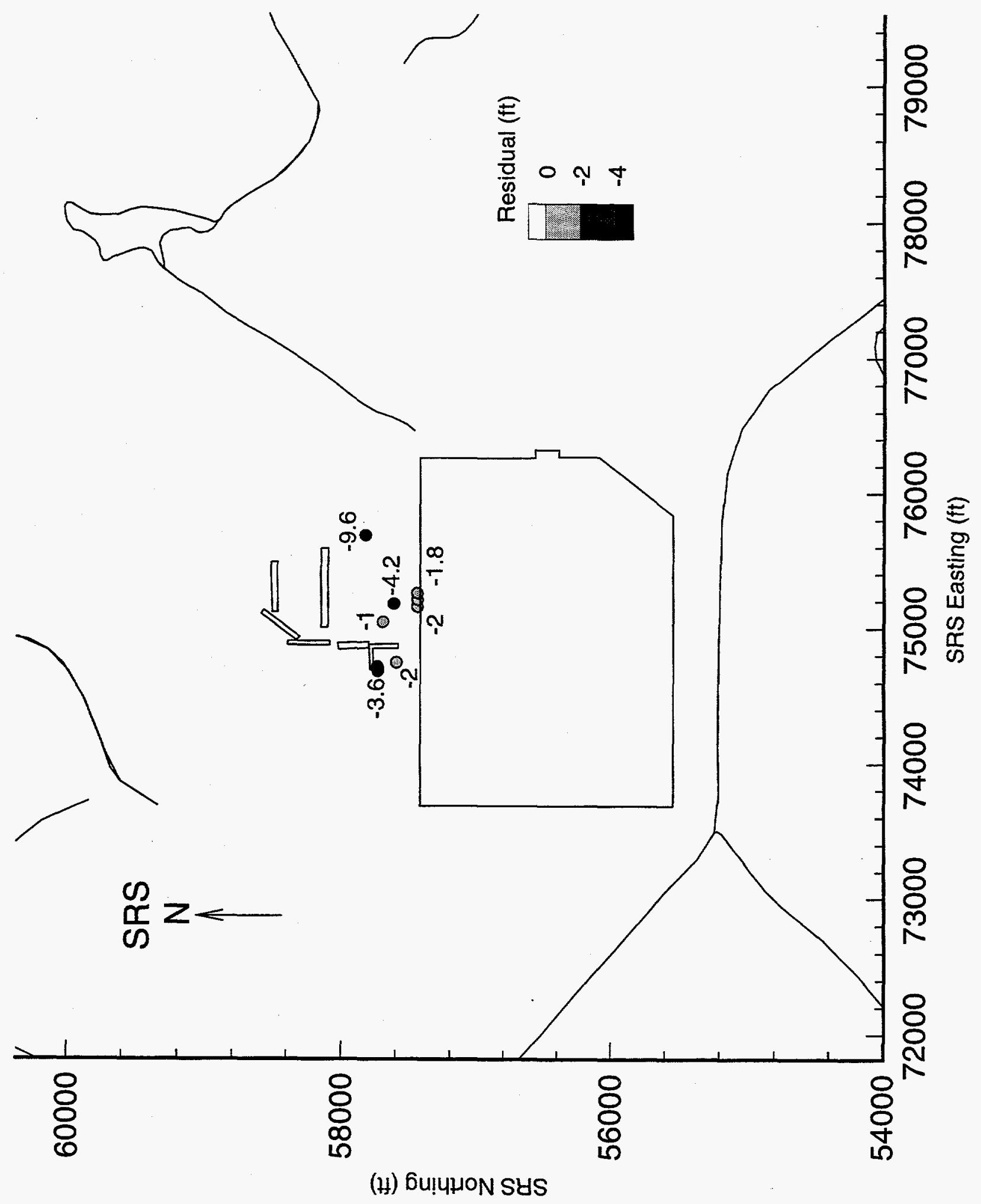




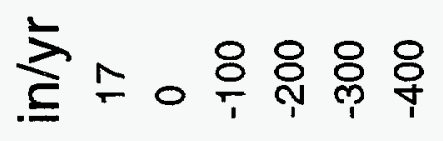

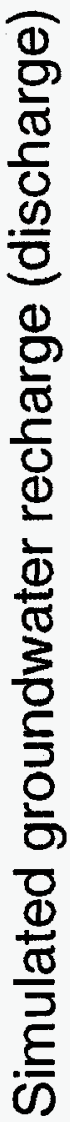

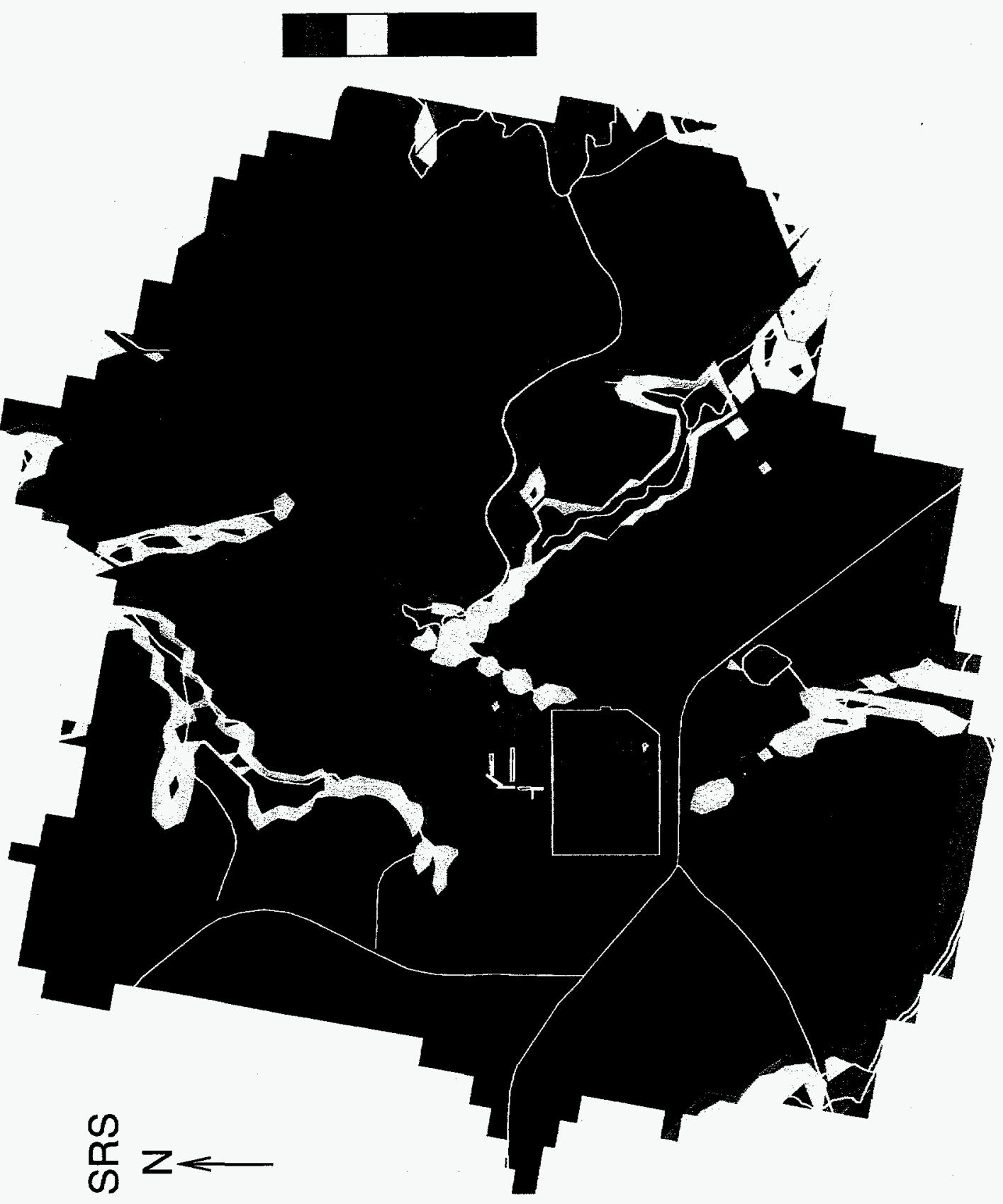

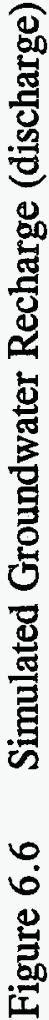




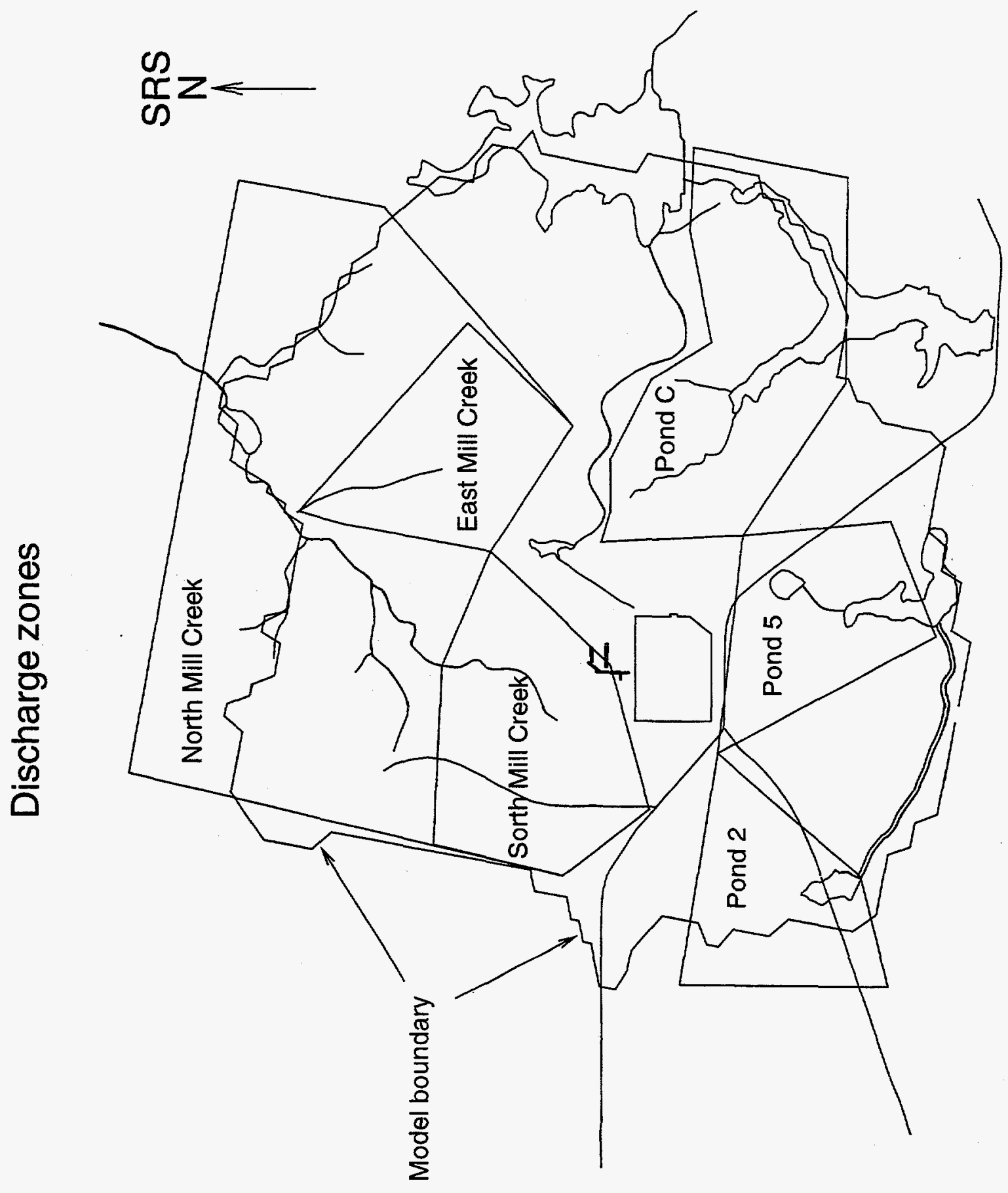

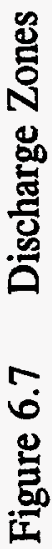




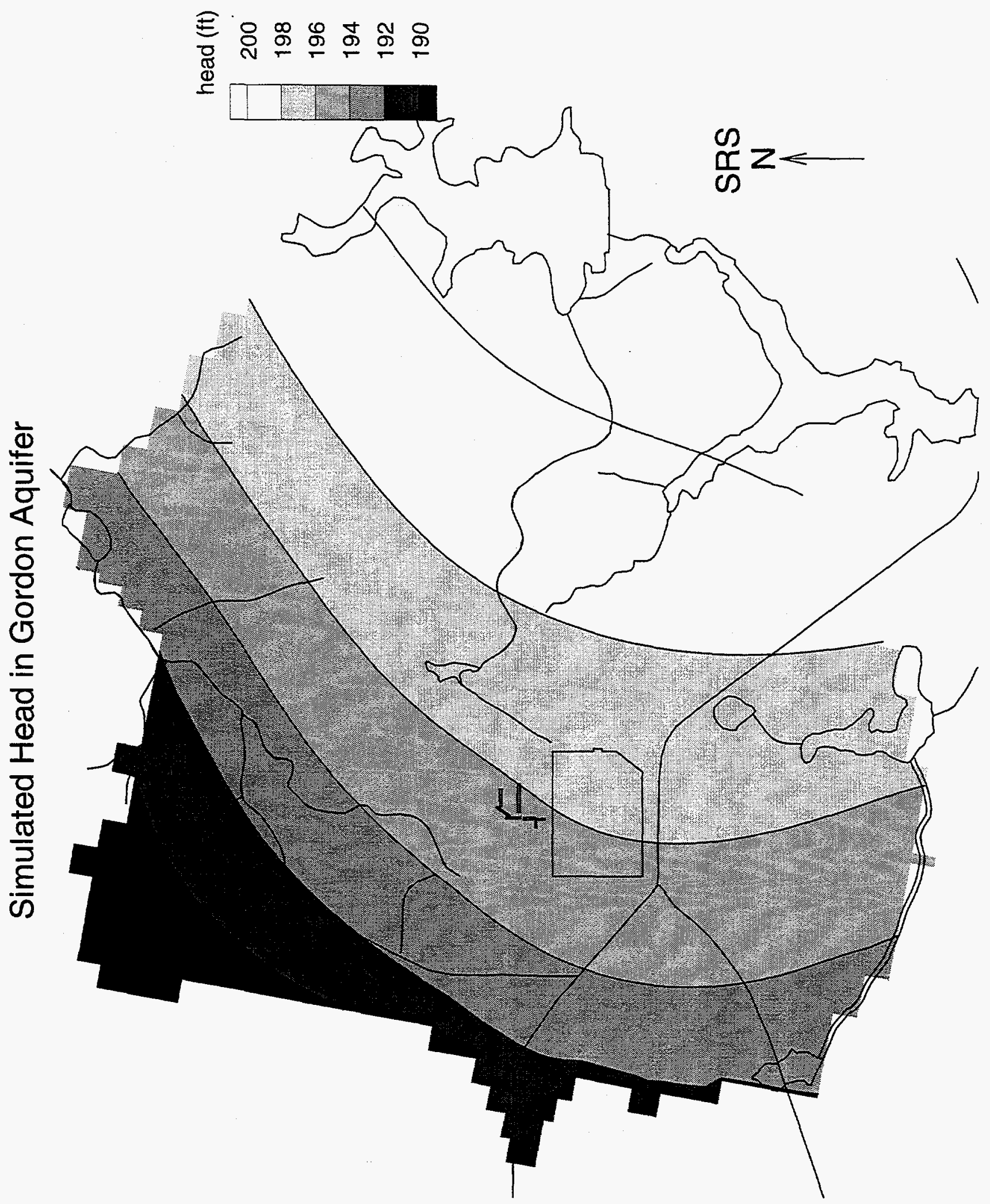




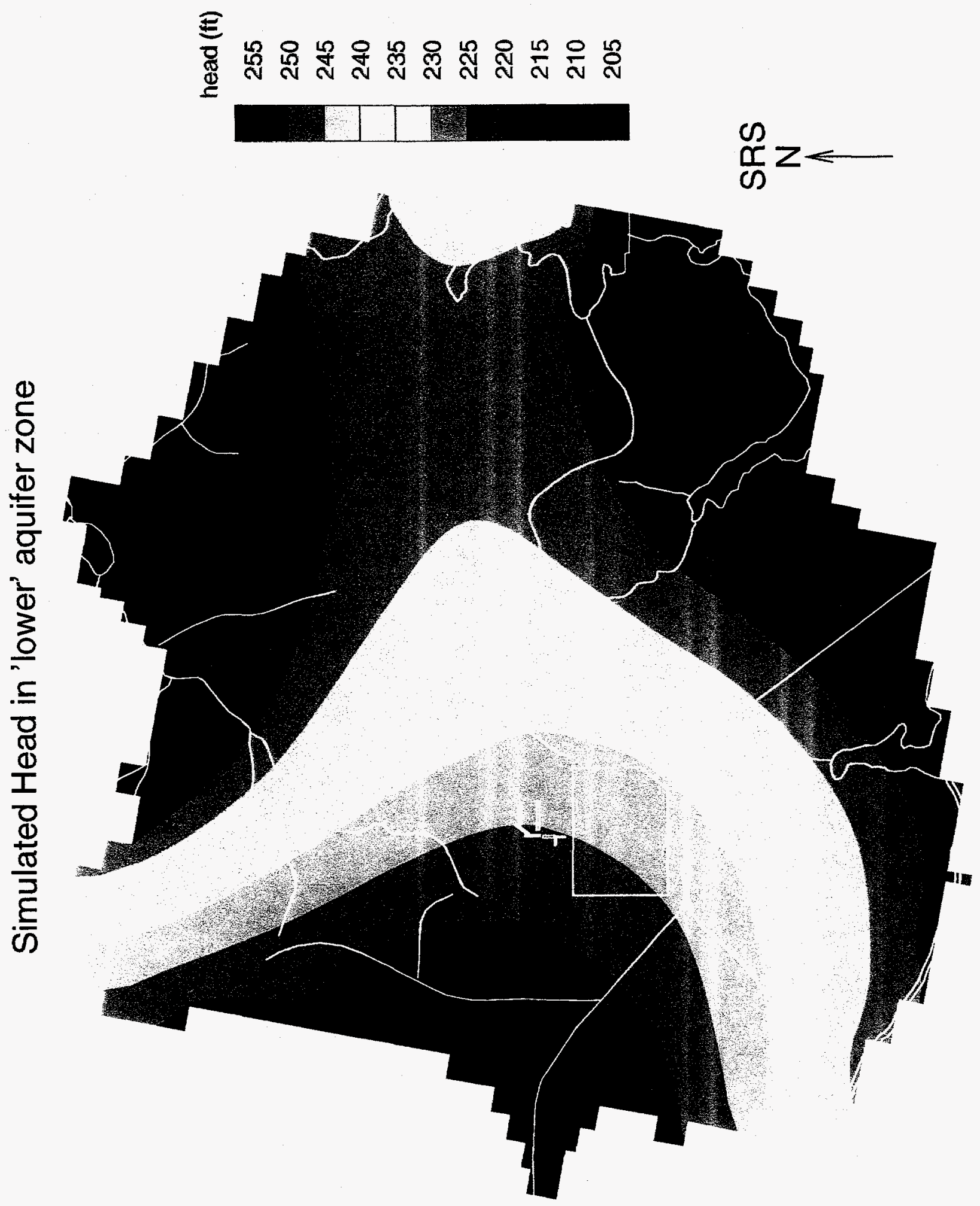

岁 


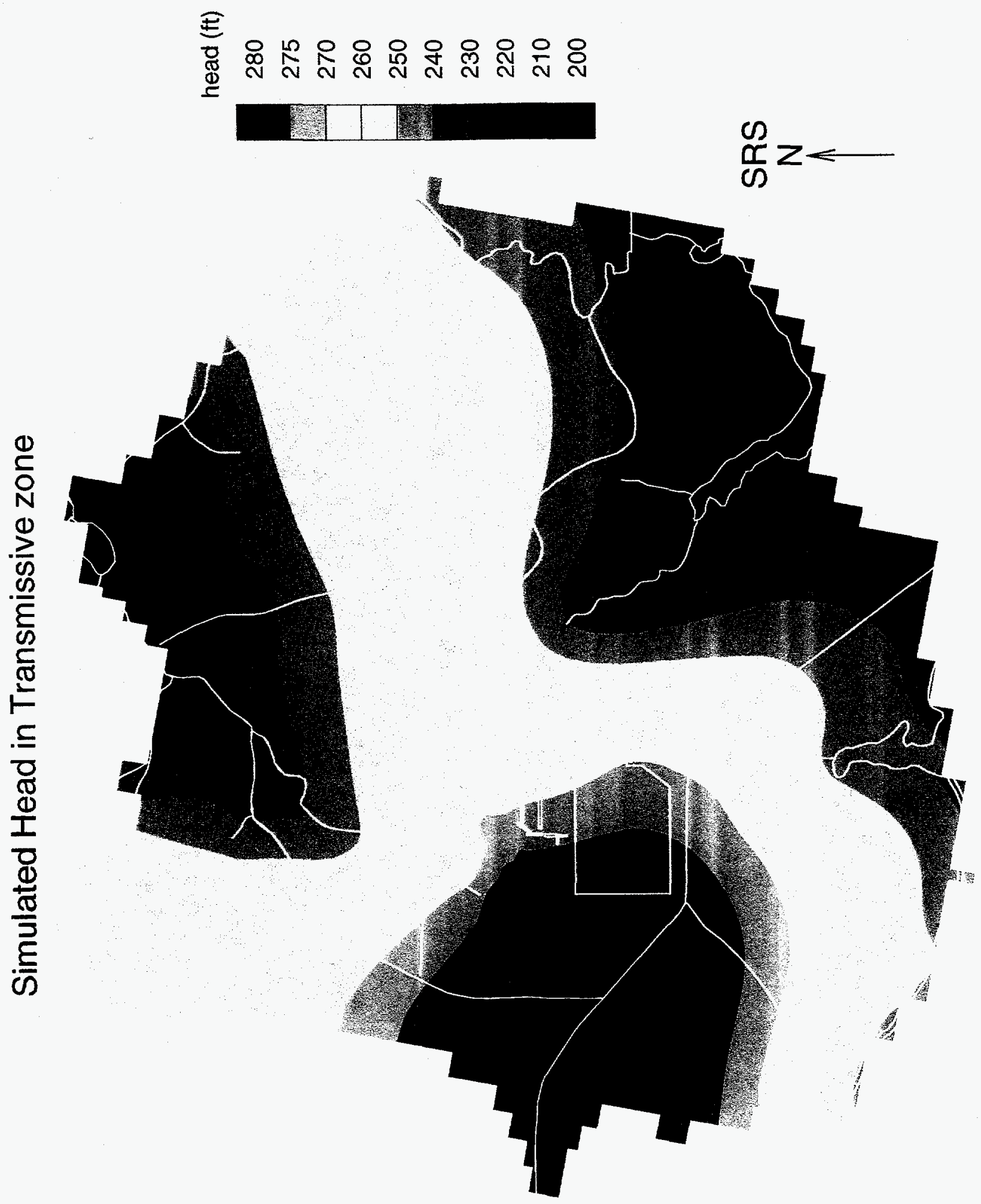




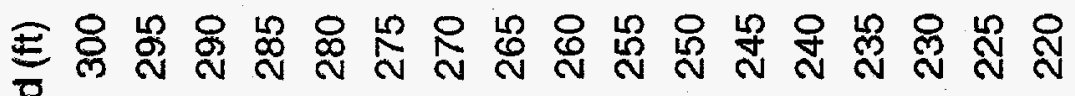

ఫु

$\stackrel{\Phi}{\Xi}$

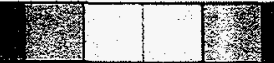

$\frac{0}{\frac{0}{0}}$

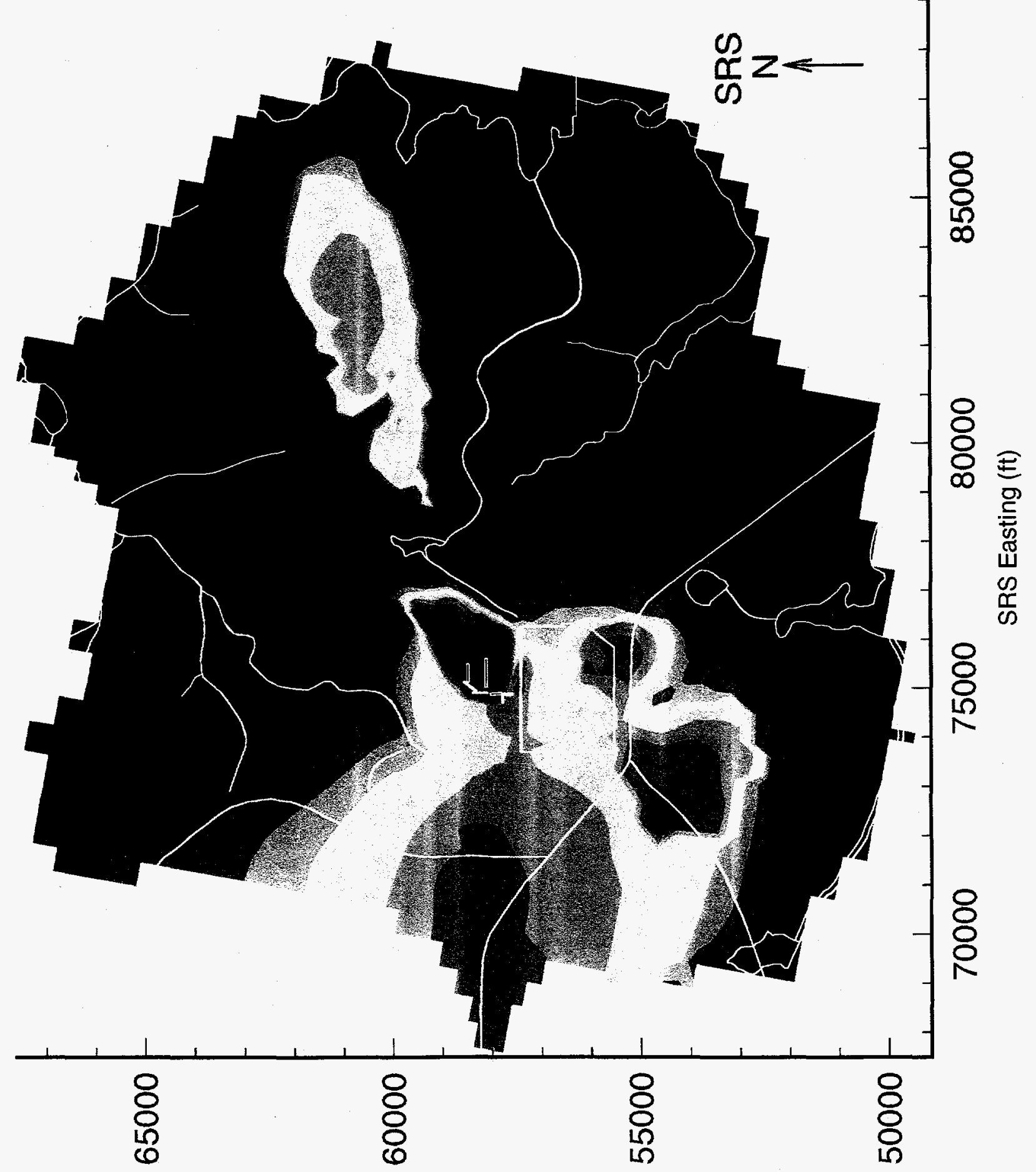



(4) 6u!YHON SUS 


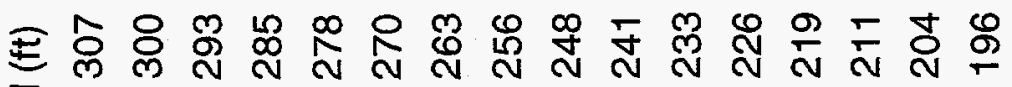

क

$\Phi$

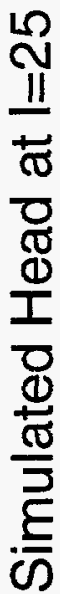

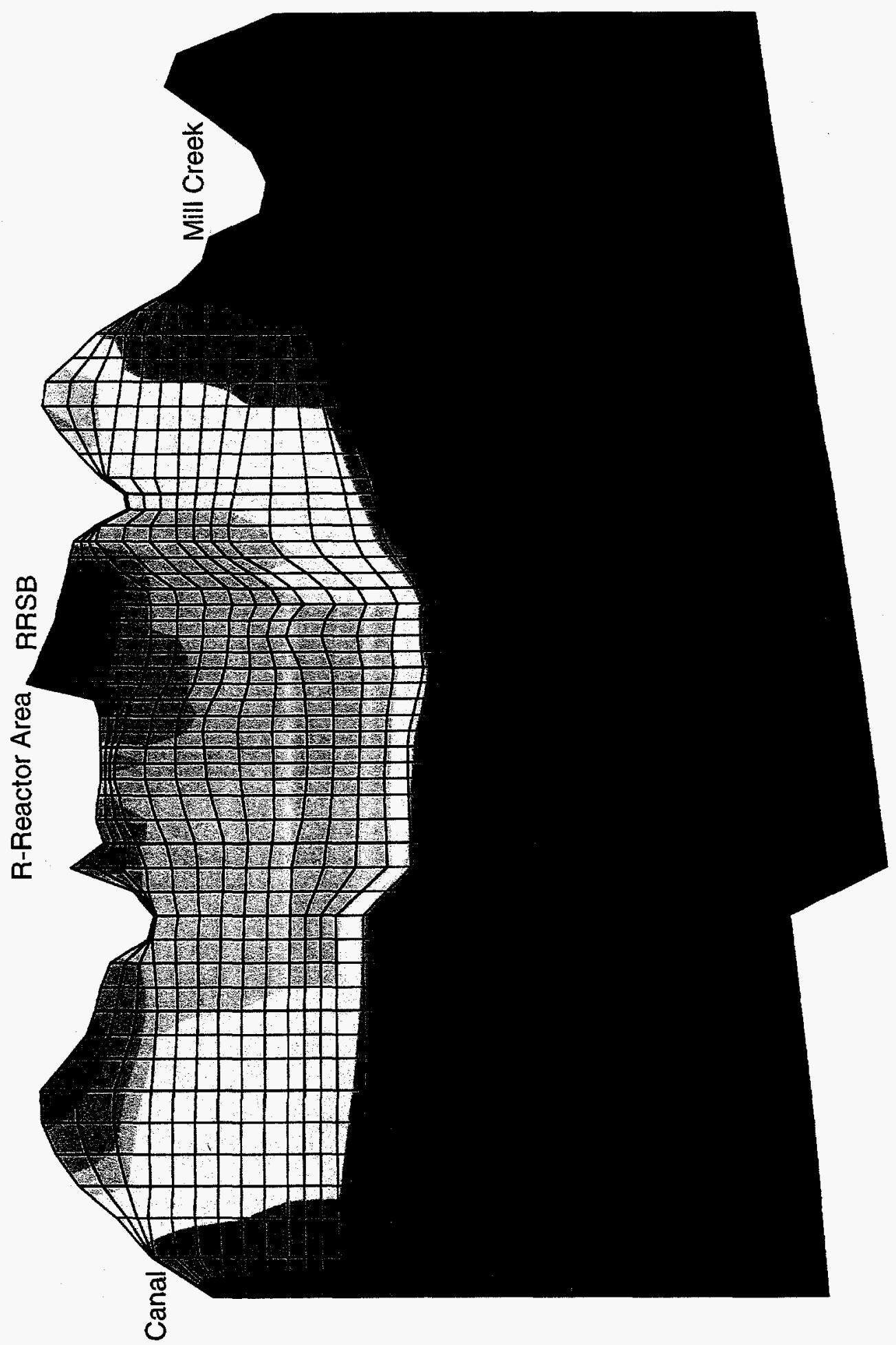

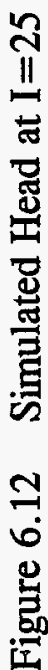




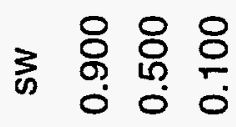

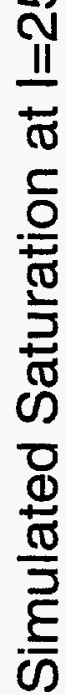

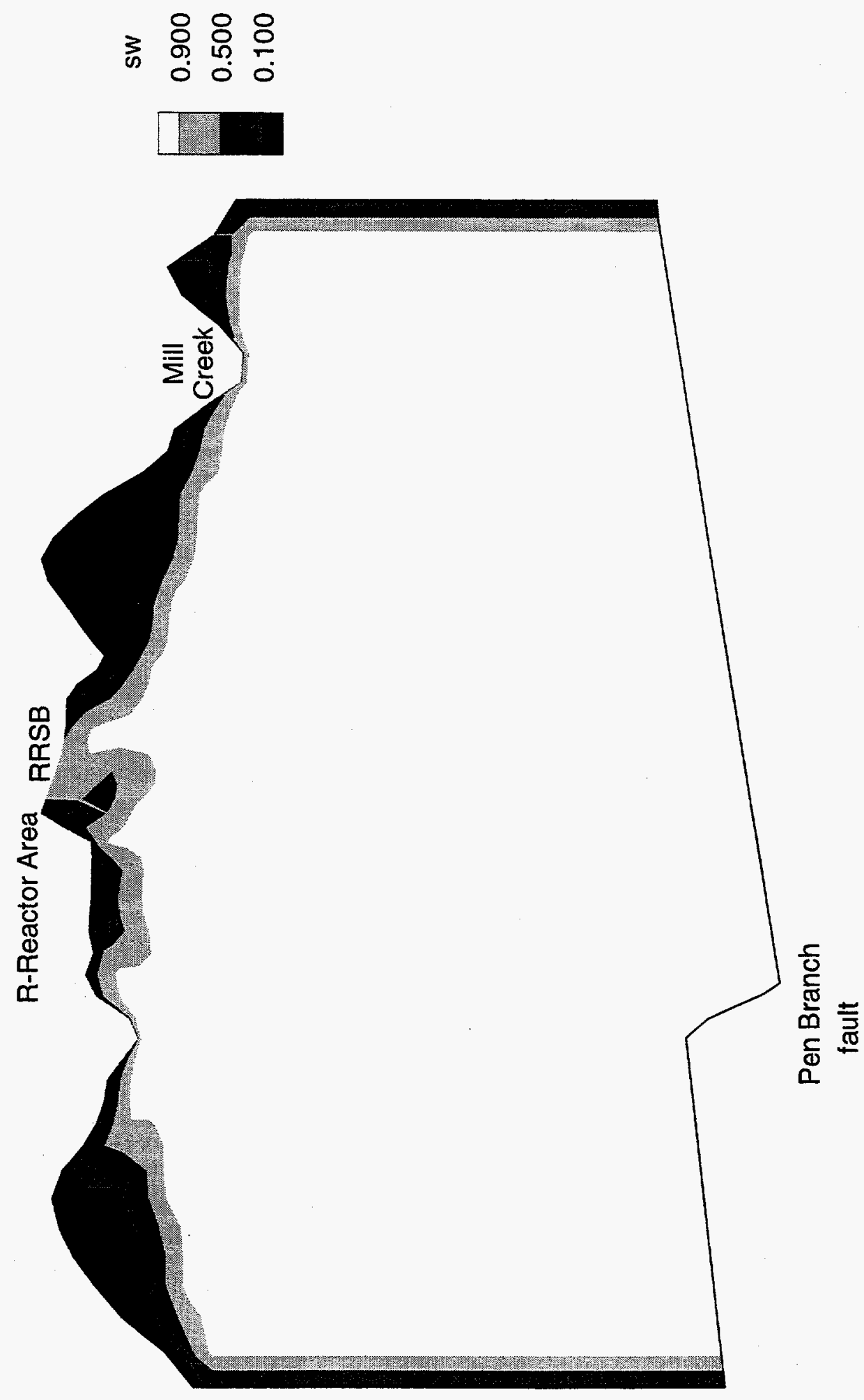




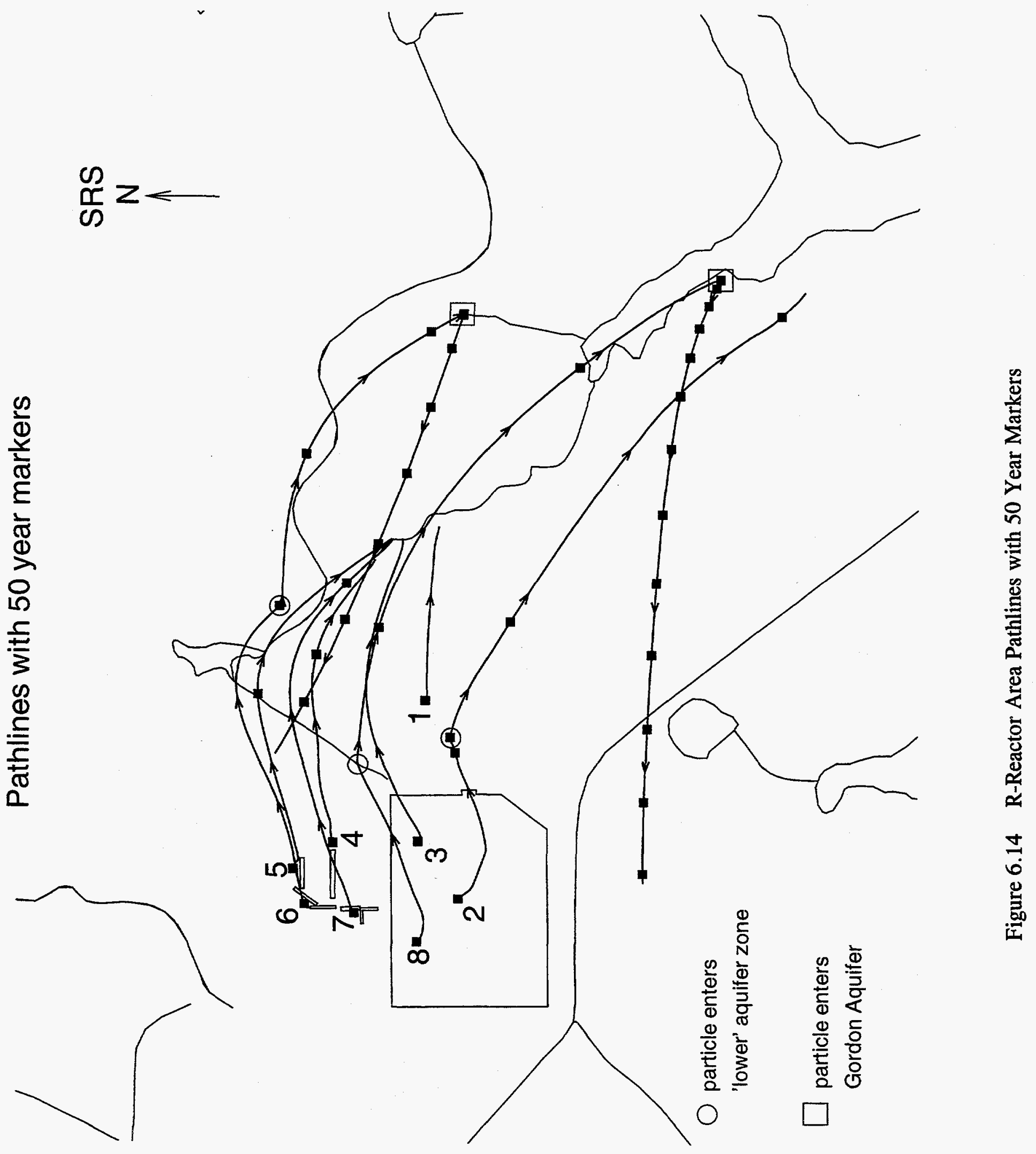




\section{Pathlines with 50 year markers (Facing North)}

$\triangle$ particle enters

'tan clay' confining zone

particle enters

'lower' aquifer zone

$\nabla$ particle enters

Gordon Confining Unit

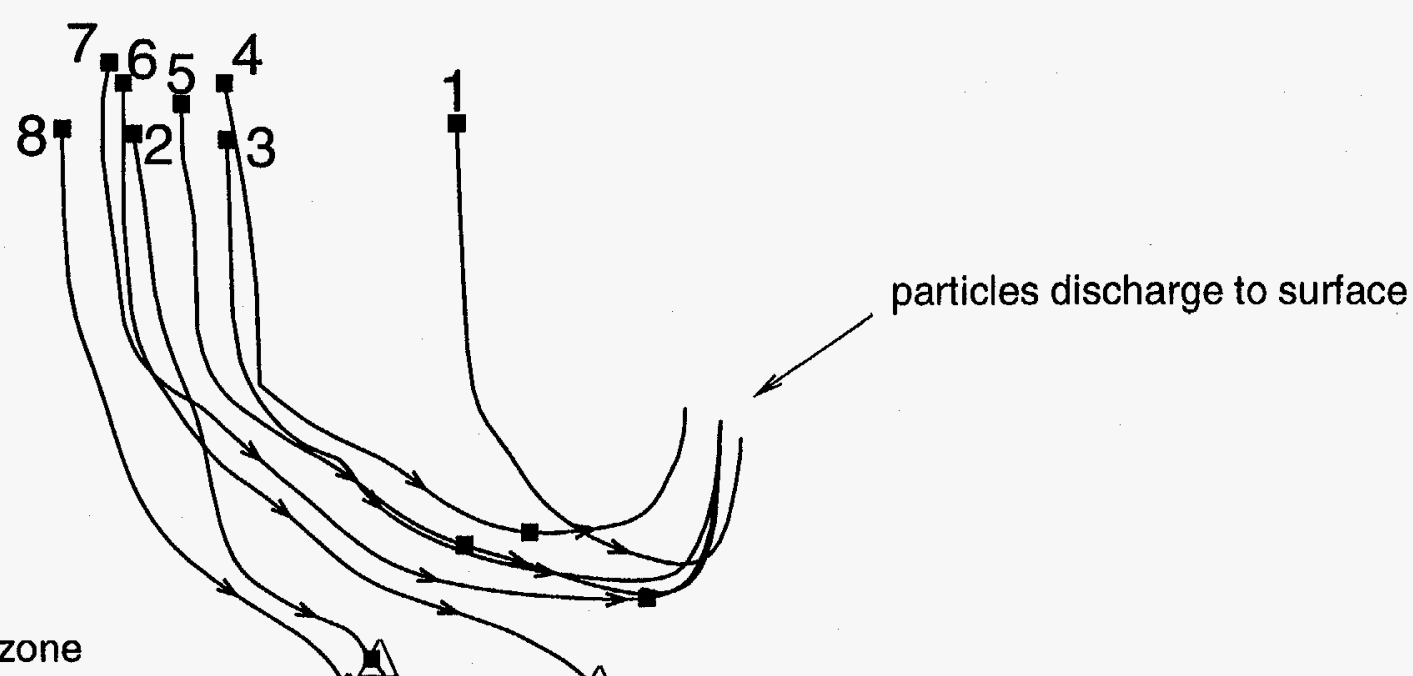




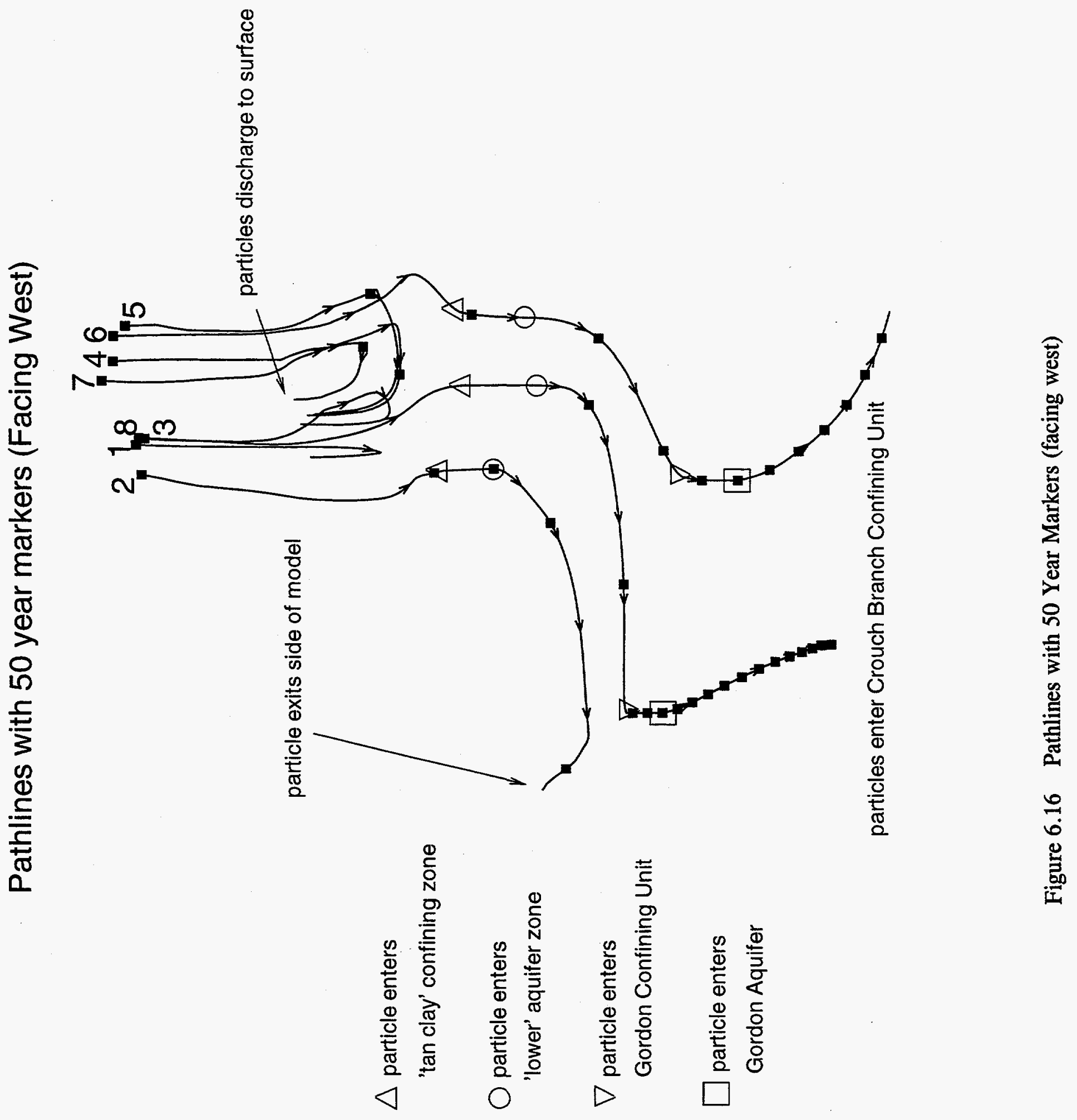


Table 4.1 Offsets South of Pen Branch Fault

\begin{tabular}{|l|c|}
\hline \multicolumn{1}{|c|}{ Unit } & Offset \\
\hline Top of Crouch Branch Confining Unit & $+40 \mathrm{ft}$ \\
\hline Gordon Confining Unit & $+40 \mathrm{ft}$ \\
\hline "tan clay" Confining Zone & $+20 \mathrm{ft}$ \\
\hline
\end{tabular}


Table 6.1 Mud Fraction Correlations and Anisotropy Ratios

\begin{tabular}{|c|c|c|c|c|}
\hline Iydrostatigraphic Unit & 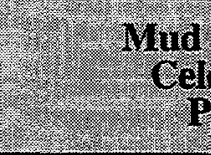 & 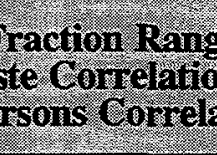 & sing & Amisongopy Ratio \\
\hline & Celeste & linear & Parsons & \\
\hline Gordon Aquifer & {$[0,0.4]$} & {$[0.4,0.46]$} & {$[0.46,1]$} & 3.6 \\
\hline Gordon Aquifer CU & {$[0,0.4]$} & {$[0.4,0.46]$} & {$[0.46,1]$} & 3.6 \\
\hline "lower" aquifer zone & {$[0,0.4]$} & {$[0.4,0.46]$} & {$[0.46,1]$} & 3.6 \\
\hline "Tan clay" confining unit & {$[0,0.15]$} & {$[0.15,0.24]$} & {$[0.24,1]$} & 3.6 \\
\hline Transmissive Zone & {$[0,0.15]$} & {$[0.15,0.24]$} & {$[0.24,1]$} & 3.6 \\
\hline "AA" horizon & {$[0,1]$} & & & 3.6 \\
\hline "A" horizon & {$[0,1]$} & & & 3.6 \\
\hline Surface Soil & {$[0,1]$} & & & 3.6 \\
\hline
\end{tabular}

Table 6.2 Friction Ratio Transition Intervals and Anisotropy Ratios

\begin{tabular}{|c|c|c|}
\hline Unit & Transition Interval & Anisotropy Ratio \\
\hline Gordon Aquifer & {$[3,5.5]$} & 3.6 \\
\hline Gordon Aquifer CU & {$[3,5.5]$} & 3.6 \\
\hline "lower" aquifer zone & {$[3,5.5]$} & 3.6 \\
\hline "Tan clay" confining unit & {$[2,3]$} & 3.6 \\
\hline Transmissive Zone & {$[2,3]$} & 3.6 \\
\hline "AA" horizon & {$[3,5.5]$} & 3.6 \\
\hline "A" horizon & {$[5.5,7]$} & 3.6 \\
\hline Surface Soil & {$[5.5,7]$} & 3.6 \\
\hline
\end{tabular}

Table 6.3 Mini-Permeability Anisotropy Ratios

\begin{tabular}{|l|c|}
\hline & Unit \\
\hline Gordon Aquifer & 3.6 \\
\hline Gordon Aquifer CU & 3.6 \\
\hline "lower" aquifer zone & 3.6 \\
\hline "Tan clay" confining unit & 200 \\
\hline Transmissive Zone & 200 \\
\hline "AA" horizon & 100 \\
\hline "A" horizon & 100 \\
\hline Surface Soil & 3.6 \\
\hline
\end{tabular}


Table 6.4 Calibration Zones

\begin{tabular}{|c|c|c|c|c|c|}
\hline Zone\# & $\begin{array}{l}\text { Aydrostratigraphic } \\
\text { Units }\end{array}$ & What & $\frac{\operatorname{Max} \mathrm{Kh}}{(\mathrm{it} / \mathrm{d})}$ & $\frac{\operatorname{MinK}}{(\mathrm{TLC})}$ & $\begin{array}{l}\text { Max Kr } \\
\text { (fta) }\end{array}$ \\
\hline 1 & $\begin{array}{l}\text { "A" and "AA" horizons, } \\
\text { surface soils }\end{array}$ & 1 & 3 & 0.01 & 0.02 \\
\hline 2 & $\begin{array}{l}\text { "A" and "AA" horizons, } \\
\text { surface soils }\end{array}$ & 1 & 3 & 0.1 & 0.2 \\
\hline 3 & transmissive zone & 0.1 & 0.5 & 0.001 & 0.005 \\
\hline
\end{tabular}

Table 6.5 Summary of Modifications to Initial Conductivity Field

\begin{tabular}{|c|c|c|c|c|c|c|}
\hline $\begin{array}{l}\text { Sylrostratigraphic } \\
\text { Gone }\end{array}$ & 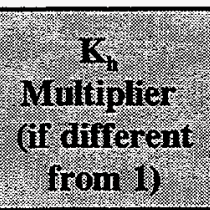 & 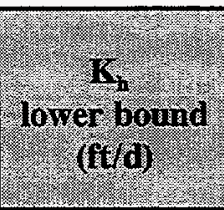 & $\begin{array}{l}\mathrm{K}_{\mathrm{h}} \\
\text { upper bound } \\
(\mathrm{ft} / \mathrm{d})\end{array}$ & $\begin{array}{l}\text { K } \\
\text { Multiplier } \\
\text { (if different } \\
\text { from 1) }\end{array}$ & 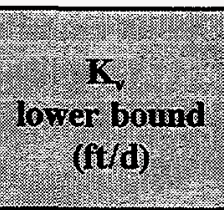 & $\begin{array}{l}\text { K, } \\
\text { upperibound } \\
(\mathrm{cta})\end{array}$ \\
\hline Gordon Aquifer & - & - & - & -- & 0.1 & - \\
\hline Gordon Confining Unit & - & - & 0.02 & - & - & 0.0001 \\
\hline "lower" aquifer zone & - & - & 20.0 & - & - & 0.4 \\
\hline $\begin{array}{l}\text { "tan clay" confining } \\
\text { zone }\end{array}$ & - & - & 1.0 & 0.5 & - & - \\
\hline Transmissive Zone & - & - & - & - & 0.1 & - \\
\hline 'AA' horizon & 2.0 & - & - & 2.0 & - & - \\
\hline ' $A$ ' horizon & 1.25 & - & - & 1.25 & - & - \\
\hline Surface Soils & 0.25 & -- & -- & 0.25 & - & -- \\
\hline
\end{tabular}


Table 6.6 Calibrated Model Average Conductivities

\begin{tabular}{|l|c|c|}
\hline \multicolumn{1}{|c|}{ OydroStratigraphic Zone } & Average $\mathrm{K}_{\text {f in Model (ft/d) }}$ & Average K, in Model (ft/d) \\
\hline Gordon Aquifer & 30.5 & 0.44 \\
\hline Gordon Confining Unit & 0.018 & 0.00018 \\
\hline "lower" aquifer zone & 18.0 & 0.22 \\
\hline "tan clay" confining zone & 0.98 & 0.015 \\
\hline Transmissive Zone & 20.7 & 0.3 \\
\hline "AA" horizon & 12.7 & 0.18 \\
\hline "A" horizon & 1.9 & 0.13 \\
\hline Surface soils & 0.73 & 0.043 \\
\hline
\end{tabular}

Table 6.7 Average residual and RMS

\begin{tabular}{|l|c|c|c|}
\hline & Nunit & Average Residual & RMS \\
\hline Gordon Aquifer & 2 & 1.7 & 1.7 \\
\hline "lower" aquifer zone & 1 & -0.1 & 0.1 \\
\hline "AA" horizon & 6 & -0.8 & 3.6 \\
\hline "A" horizon & 36 & 1.3 & 3.8 \\
\hline Surface soil & 10 & -3.2 & 4.0 \\
\hline
\end{tabular}


Table 6.8 Comparison of Measured and Simulated Stream Baseflow

\begin{tabular}{|c|c|c|}
\hline 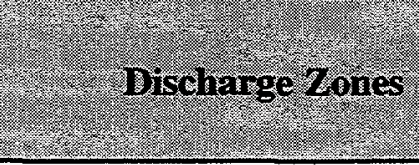 & $\begin{array}{l}\text { Estimated Baseflow } \\
\text { Contmbution from R-Reactor } \\
\text { Area }\left(\mathrm{d}^{3} / \mathrm{s}\right)\end{array}$ & 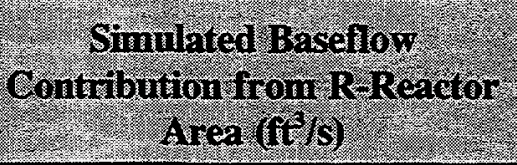 \\
\hline North Mill Creek & - & 2.3 \\
\hline South Mill Creek & $0.3-0.4$ & 0.25 \\
\hline East Mill Creek & - & 0.33 \\
\hline Pond C Tributaries & - & 2.4 \\
\hline Pond 5 Tributaries & - & 0.33 \\
\hline Pond 2 Tributaries & -- & 0.18 \\
\hline
\end{tabular}

Table 6.9 Seeds for 3-D Pathlines

\begin{tabular}{|c|c|c|c|}
\hline Seed \# & SRS Easting (ft) & SRS Northing (it) & Dlevation (fi) \\
\hline 1 & 77400 & 57000 & 290 \\
\hline 2 & 74990 & 56620 & 288 \\
\hline 3 & 75690 & 57100 & 287 \\
\hline 4 & 75680 & 58120 & 298 \\
\hline 5 & 75360 & 58590 & 294 \\
\hline 6 & 74930 & 58460 & 298 \\
\hline 7 & 74820 & 57870 & 302 \\
\hline 8 & 74470 & 57120 & 289 \\
\hline
\end{tabular}




\section{Appendix A \\ Hydraulic Head Data and Residuals}


Monitoring Well Data

\begin{tabular}{|c|c|c|c|c|c|c|c|}
\hline Well ID & $\begin{array}{c}\text { SRS } \\
\text { Easting (ft) }\end{array}$ & $\begin{array}{c}\text { SRS } \\
\text { Northing (ft) }\end{array}$ & $\begin{array}{c}\text { Bottom } \\
\text { of screen ( } \mathrm{ft} \text { ) }\end{array}$ & $\begin{array}{c}\text { Top } \\
\text { of screen }(f t)\end{array}$ & $\begin{array}{l}\text { Average } \\
\text { Head (ft) }\end{array}$ & $\begin{array}{l}\text { Fact (ft) } \\
\text { Run } 9 \mathrm{C}\end{array}$ & Residual (ft) \\
\hline \multicolumn{8}{|c|}{ Gordon Aquifer } \\
\hline P 20B & 76816.7 & 56081.7 & 28.1 & 48.1 & 195.2 & 197.1 & 1.9 \\
\hline RCP 1A & 74238.3 & 56968.1 & 46.8 & 56.8 & 194.2 & 195.7 & 1.5 \\
\hline \multicolumn{8}{|c|}{ Lower Aquifer Zone } \\
\hline P 20C & 76814.0 & 56067.7 & 138.0 & 148.0 & 238.5 & 238.4 & -0.1 \\
\hline \multicolumn{8}{|c|}{ AA' Horizon } \\
\hline P 200 & 76784.5 & 56075.2 & 225.4 & 244.4 & 269.2 & 267.5 & -1.7 \\
\hline RSC 9 & 74565.3 & 59241.2 & 251.6 & 271.6 & 271.8 & 278.4 & 6.6 \\
\hline RSE 24 & 74638.9 & 57370.4 & 237.6 & 257.6 & 279.5 & 279.2 & -0.3 \\
\hline RSF 1 & 74869.4 & 58505.3 & 228.8 & 238.8 & 277.4 & 274.6 & -2.8 \\
\hline RSF 2 & 74628.6 & 57670.4 & 224.8 & 235.3 & 278.1 & 275.8 & -2.3 \\
\hline RSF 3 & 75206.7 & 57621.4 & 229.8 & 239.8 & 279.4 & 274.9 & -4.5 \\
\hline \multicolumn{8}{|c|}{ A'Horizon } \\
\hline RAC 1 & 74570.7 & 55107.3 & 247.3 & 277.3 & 273.7 & 276.6 & 2.9 \\
\hline RAC 2 & 74555.5 & 55026.3 & 243.4 & 273.4 & 272.5 & 275.9 & 3.4 \\
\hline RAC 3 & 74667.5 & 55015.3 & 242.3 & 272.3 & 272.2 & 275.5 & 3.3 \\
\hline RAC 4 & 74588.8 & 54984.0 & 238.2 & 268.2 & 271.5 & 275.3 & 3.8 \\
\hline RCP 1D & 74223.5 & 56967.9 & 261.3 & 281.3 & 281.6 & 282.5 & 0.9 \\
\hline RDB 1D & 74844.5 & 57097.3 & 265.5 & 285.5 & 285.8 & 282.5 & -3.3 \\
\hline RDB 2D & 74782.2 & 56879.8 & 265.7 & 285.7 & 285.0 & 280.1 & -4.9 \\
\hline RDB 3D & 74899.0 & 56881.9 & 265.8 & 285.8 & 282.8 & 279.3 & -3.5 \\
\hline RRP 1 & 75634.6 & 54563.5 & 242.4 & 272.4 & 265.6 & 271.7 & 6.1 \\
\hline RRP 2 & 75829.8 & 54468.3 & 242.5 & 272.5 & 264.7 & 270.8 & 6.1 \\
\hline RRP 3 & 75853.0 & 54303.0 & 238.1 & 268.1 & 264.1 & 269.0 & 4.9 \\
\hline RRP 4 & 75723.3 & 54294.5 & 238.3 & 268.3 & 263.8 & 269.6 & 5.8 \\
\hline RSA 9 & 75616.2 & 58123.8 & 264.6 & 284.5 & 283.0 & 276.3 & -6.7 \\
\hline RSA 10 & 75389.3 & 58172.8 & 268.8 & 288.7 & 280.8 & 280.5 & -0.3 \\
\hline RSC 2 & 74378.6 & 58543.0 & 261.9 & 281.9 & 278.3 & 280.6 & 2.3 \\
\hline RSC 3 & 74699.7 & 58724.7 & 258.6 & 278.6 & 276.7 & 281.0 & 4.3 \\
\hline RSC 4 & 75097.8 & 58900.3 & 268.6 & 288.6 & 280.6 & 280.5 & -0.1 \\
\hline RSC 5 & 75484.9 & 58901.2 & 258.3 & 278.3 & 274.2 & 276.9 & 2.7 \\
\hline RSC 10 & 75470.0 & 59542.7 & 255.5 & 275.5 & 271.9 & 274.2 & 2.3 \\
\hline RSD 1 & 75035.1 & 57440.8 & 267.9 & 287.7 & 286.4 & 285.2 & -1.2 \\
\hline RSD 3 & 74702.3 & 57451.6 & 269.3 & 289.1 & 286.8 & 284.6 & -2.2 \\
\hline RSD 7 & 75178.4 & 57394.3 & 267.3 & 287.3 & 285.2 & 285.4 & 0.2 \\
\hline RSD 8 & 75229.6 & 57394.0 & 267.3 & 287.3 & 285.4 & 285.3 & -0.1 \\
\hline RSD 9 & 75185.9 & 57245.6 & 251.7 & 271.7 & 283.8 & 279.6 & -4.2 \\
\hline RSE 3A & 74931.2 & 57445.8 & 268.2 & 288.0 & 285.2 & 285.0 & -0.2 \\
\hline RSE 4A & 75101.1 & 57528.4 & 260.6 & 270.6 & 286.6 & 282.6 & -4.0 \\
\hline RSE 7 & 74783.7 & 58481.5 & 266.5 & 286.3 & 280.6 & 281.5 & 0.9 \\
\hline RSE 8 & 74869.4 & 58538.8 & 271.2 & 291.0 & 284.0 & 282.8 & -1.2 \\
\hline RSE 9 & 74971.1 & 58463.3 & 266.7 & 286.7 & 279.2 & 280.8 & 1.6 \\
\hline RSE 10 & 74848.3 & 58420.7 & 270.7 & 290.5 & 281.7 & 282.7 & 1.0 \\
\hline RSE 18 & 74839.5 & 58247.2 & 268.1 & 288.1 & 279.6 & 282.4 & 2.8 \\
\hline RSE 19 & 74791.2 & 58318.4 & 262.5 & 282.5 & 280.9 & 281.2 & 0.3 \\
\hline RSE 25 & 74544.5 & 55824.5 & 237.5 & 257.5 & 275.3 & 278.0 & 2.7 \\
\hline RBP 1D & 77582.0 & 57217.0 & 243.0 & 253.0 & 254.5 & 260.6 & 6.1 \\
\hline RBP 2D & 77510.0 & 56862.0 & 243.0 & 253.0 & 253.0 & 261.2 & 8.2 \\
\hline RBP 3D & 77206.0 & 56828.0 & 248.0 & 258.0 & 258.0 & 264.5 & 6.5 \\
\hline \multicolumn{8}{|c|}{ Surface Soils } \\
\hline RSB 7 & 75044.3 & 57692.8 & 272.7 & 292.6 & 285.9 & 284.9 & -1.0 \\
\hline RSB 8 & 75178.2 & 57612.9 & 274.3 & 294.3 & 288.0 & 283.8 & -4.2 \\
\hline RSC 8 & 75684.0 & 57818.1 & 271.3 & 299.3 & 293.6 & 284.0 & -9.6 \\
\hline RSD 4 & 75154.6 & 57441.4 & 270.6 & 290.6 & 288.5 & 286.5 & -2.0 \\
\hline RSD 5 & 75207.0 & 57439.9 & 269.6 & 289.6 & 287.2 & 285.7 & -1.5 \\
\hline RSD 6 & 75256.6 & 57441.3 & 270.1 & 290.1 & 287.1 & 285.3 & -1.8 \\
\hline RSE 1A & 74712.7 & 57734.5 & 274.8 & 294.8 & 288.6 & 285.3 & -3.3 \\
\hline RSE 1B & 74698.1 & 57731.4 & 275.7 & 295.7 & 288.8 & 285.3 & -3.5 \\
\hline RSE $1 \mathrm{C}$ & 74684.1 & 57730.8 & 268.5 & 288.5 & 288.7 & 285.1 & -3.6 \\
\hline RSE 2 & 74743.5 & 57594.9 & 269.7 & 289.5 & 286.7 & 284.7 & -2.0 \\
\hline
\end{tabular}




\section{Appendix B \\ Borehole and CPT Data}


Borehole Data

\begin{tabular}{|c|c|c|}
\hline Well ID & SRS Dasting & SRS Northing \\
\hline N13 & 70856.20 & 66632.10 \\
\hline P20 & 76784.50 & 56075.20 \\
\hline RBW2CL & 71795.10 & 58712.00 \\
\hline RBW2CU & 71786.00 & 58715.30 \\
\hline RCH1 & 74294.90 & 59123.40 \\
\hline RCP1A & 74238.30 & 56968.10 \\
\hline RPC1CL & 74261.90 & 57923.30 \\
\hline RPC2CL & 76120.70 & 58077.20 \\
\hline
\end{tabular}

\section{CPT Data}

\begin{tabular}{|l|l|l|l|}
\hline WeII 1D & SRS Easting & SRS Northing & Performed by \\
\hline RBW1 & 74232.00 & 62042.50 & Fugro \\
\hline RBW2CL & 71795.10 & 58712.00 & Fugro \\
\hline RCH1 & 74294.90 & 59123.40 & Fugro \\
\hline RPC1CL & 74261.90 & 57923.30 & Fugro \\
\hline RPC2CL & 76120.70 & 58077.20 & Fugro \\
\hline RPT1 & 75568.70 & 55946.90 & ARA \\
\hline RPT2 & 76333.40 & 56881.70 & ARA \\
\hline RPT3 & 75939.70 & 57479.80 & Fugro \\
\hline RPT5 & 76279.40 & 58755.70 & Fugro \\
\hline RPT6 & 75363.40 & 59311.20 & Fugro \\
\hline RPT7 & 75014.33 & 57848.28 & ARA \\
\hline RPT8 & 75404.33 & 58206.43 & ARA \\
\hline RPT9 & 75268.76 & 58625.32 & ARA \\
\hline
\end{tabular}




\section{Appendix C \\ Core Mud Fraction Plots}




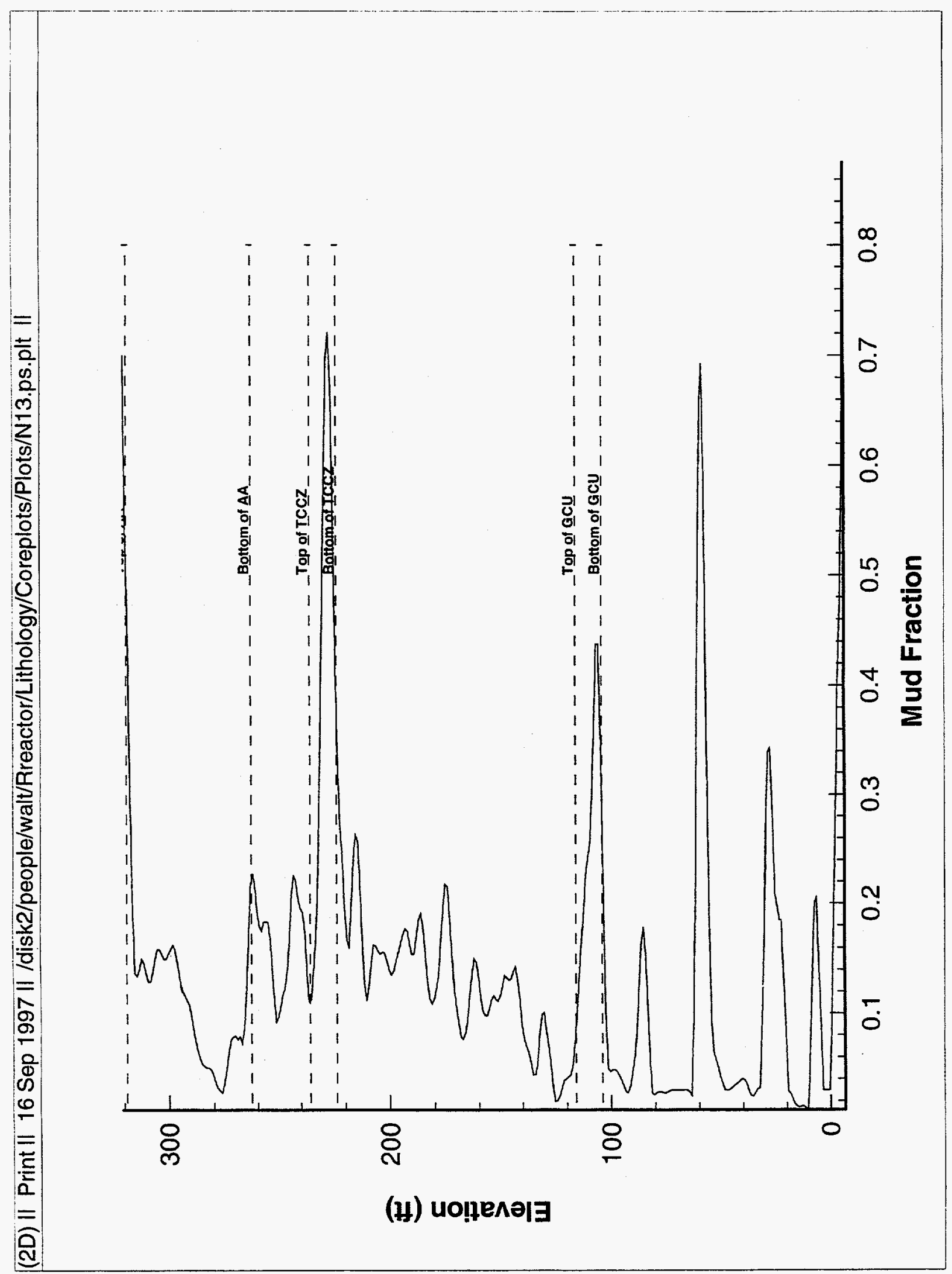




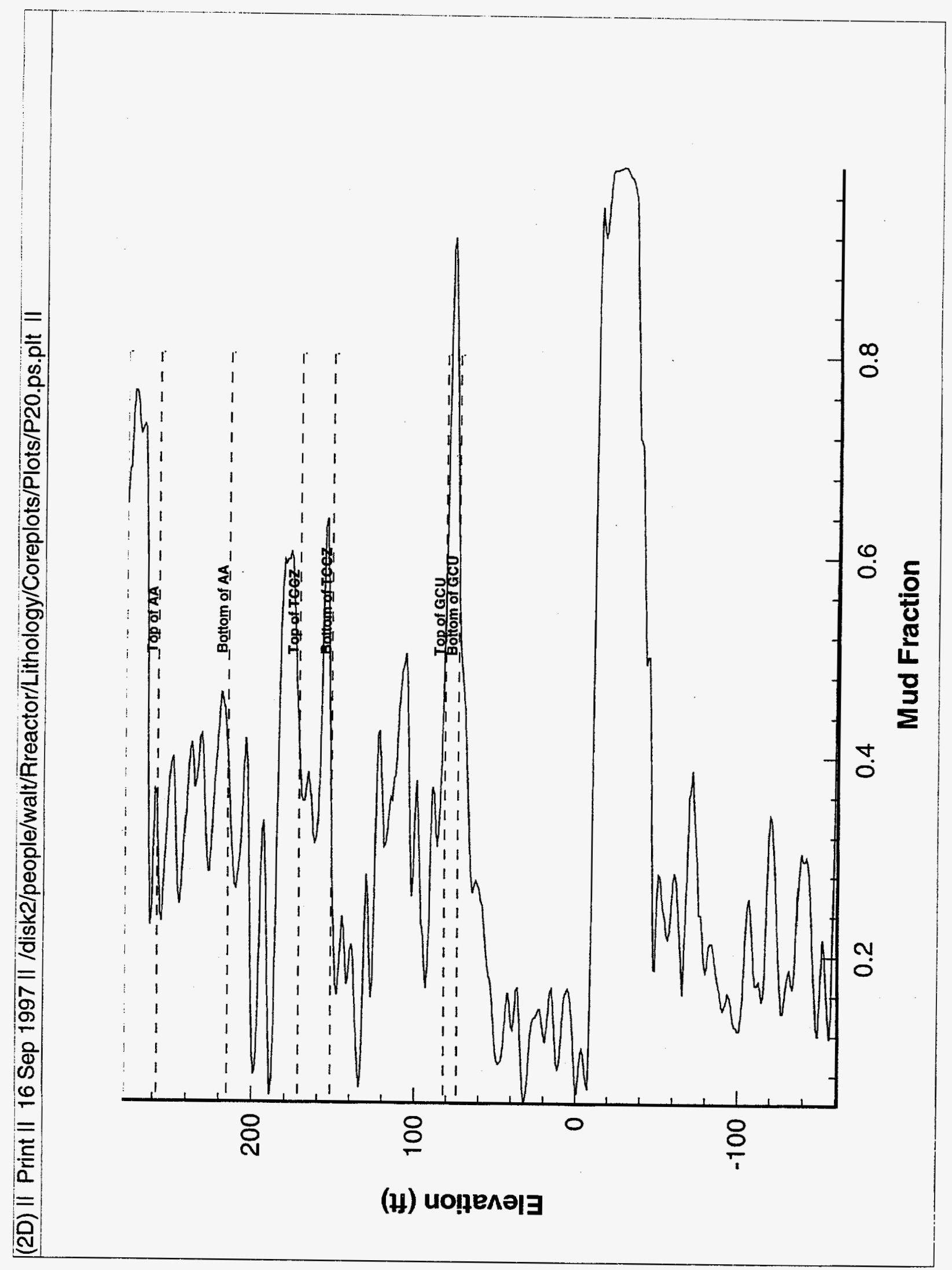




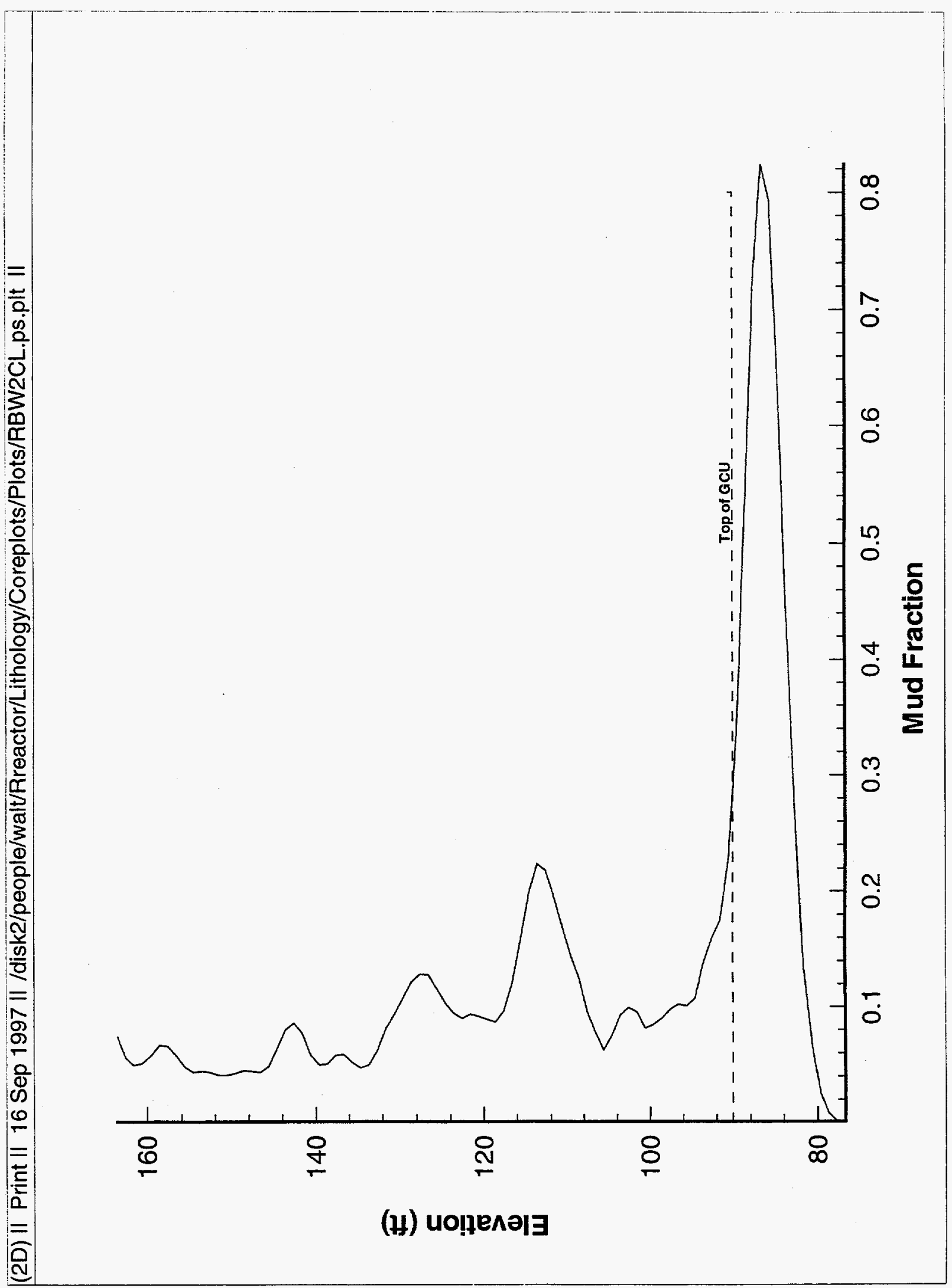




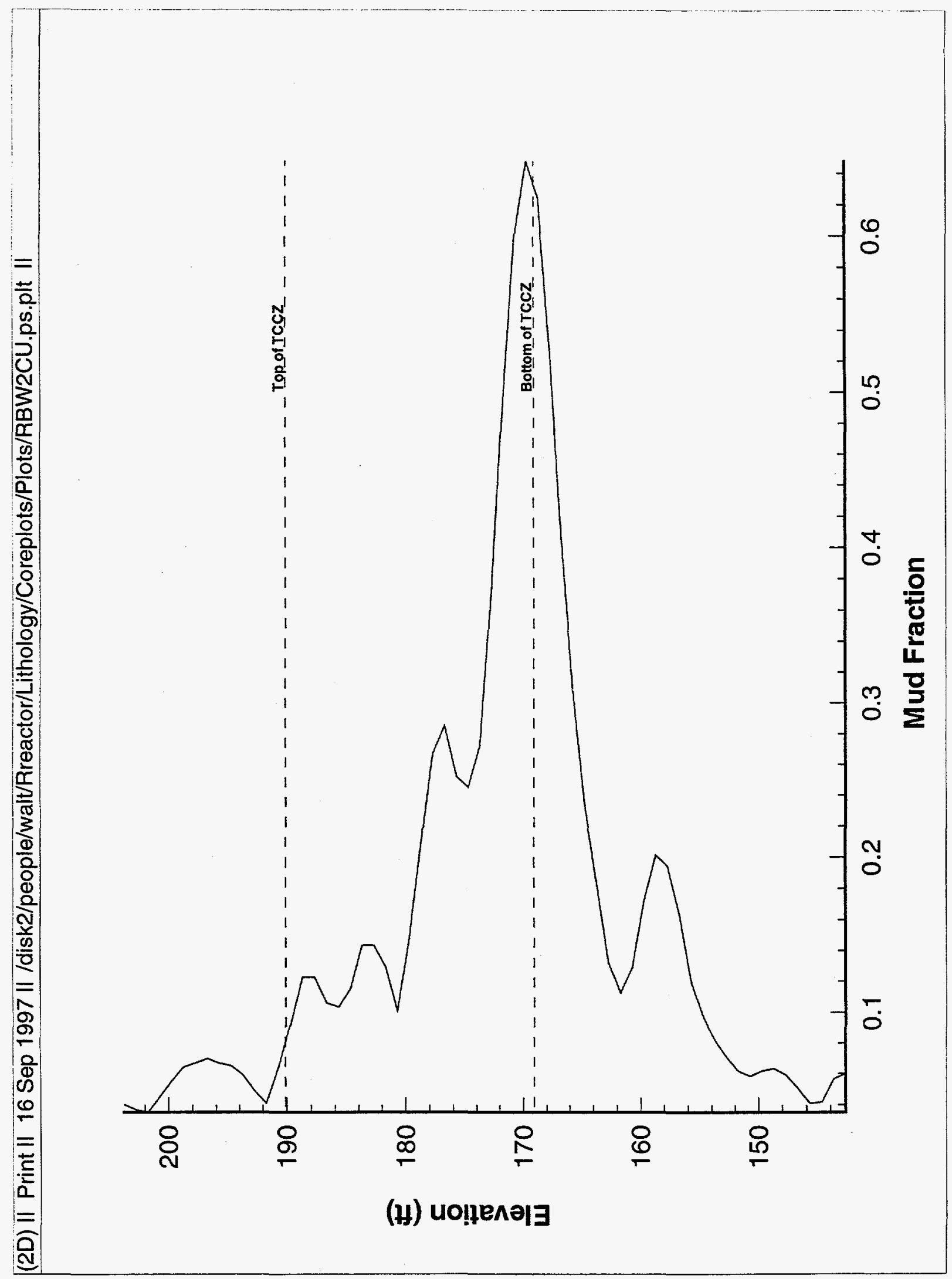




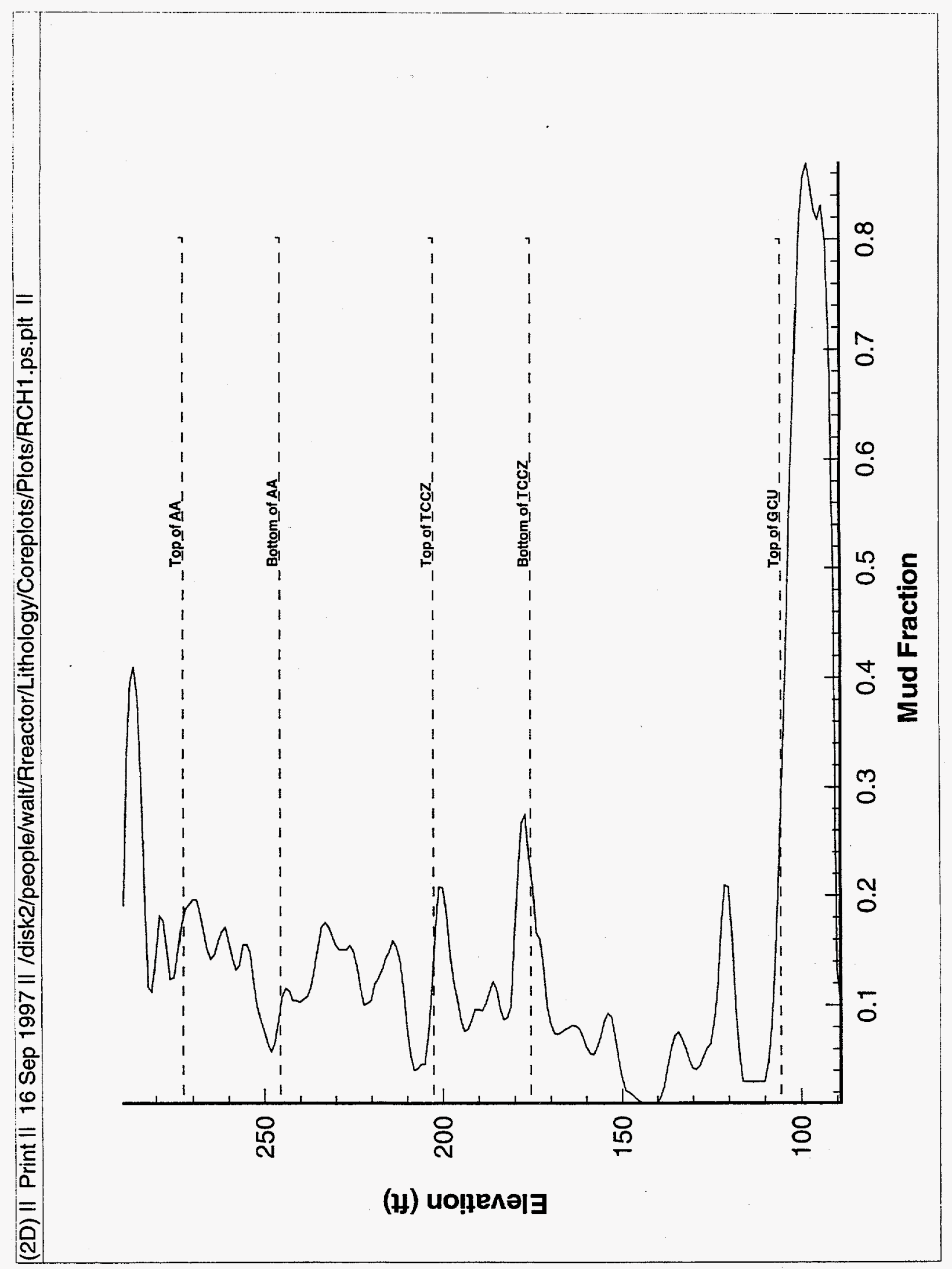




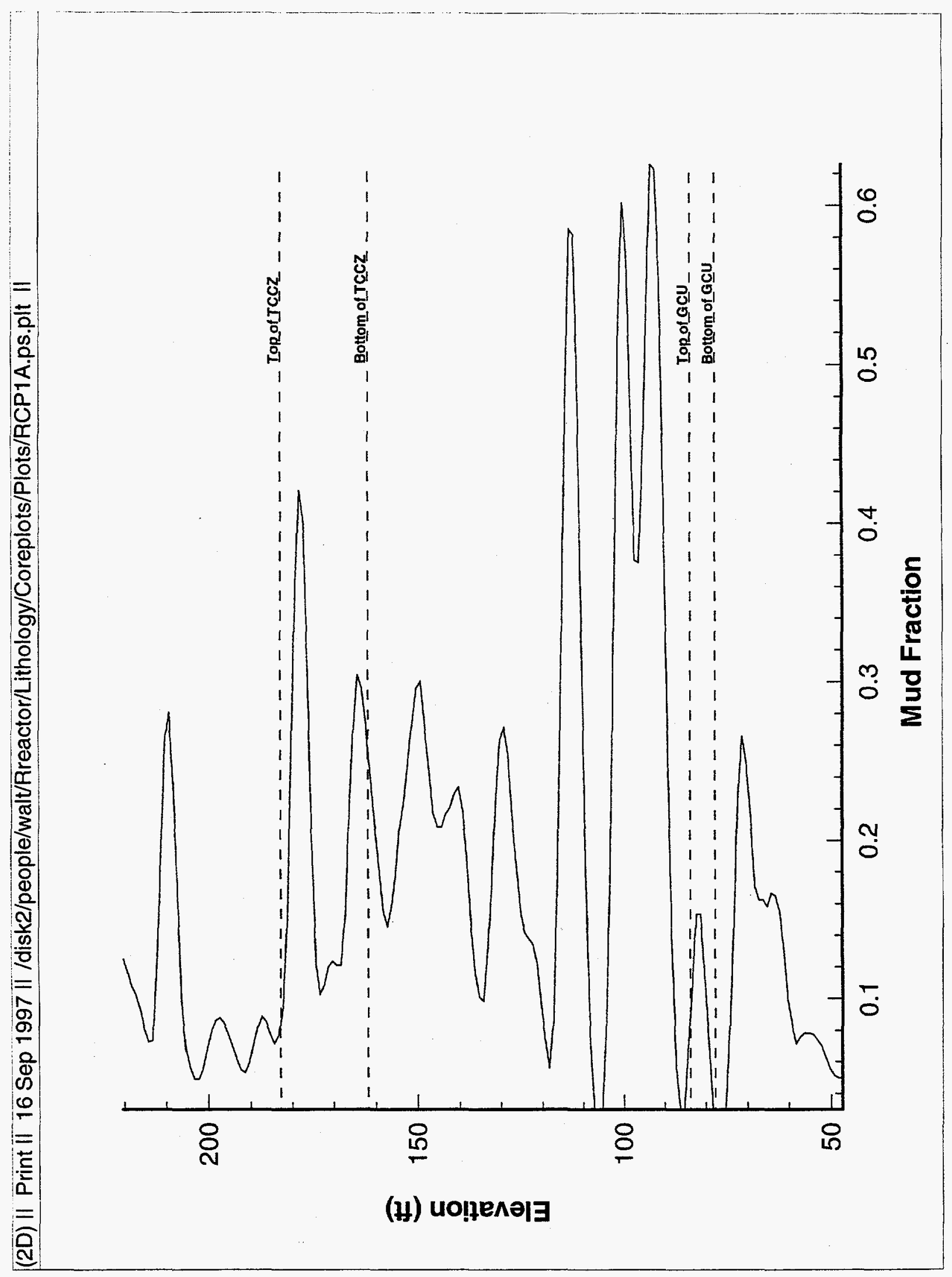




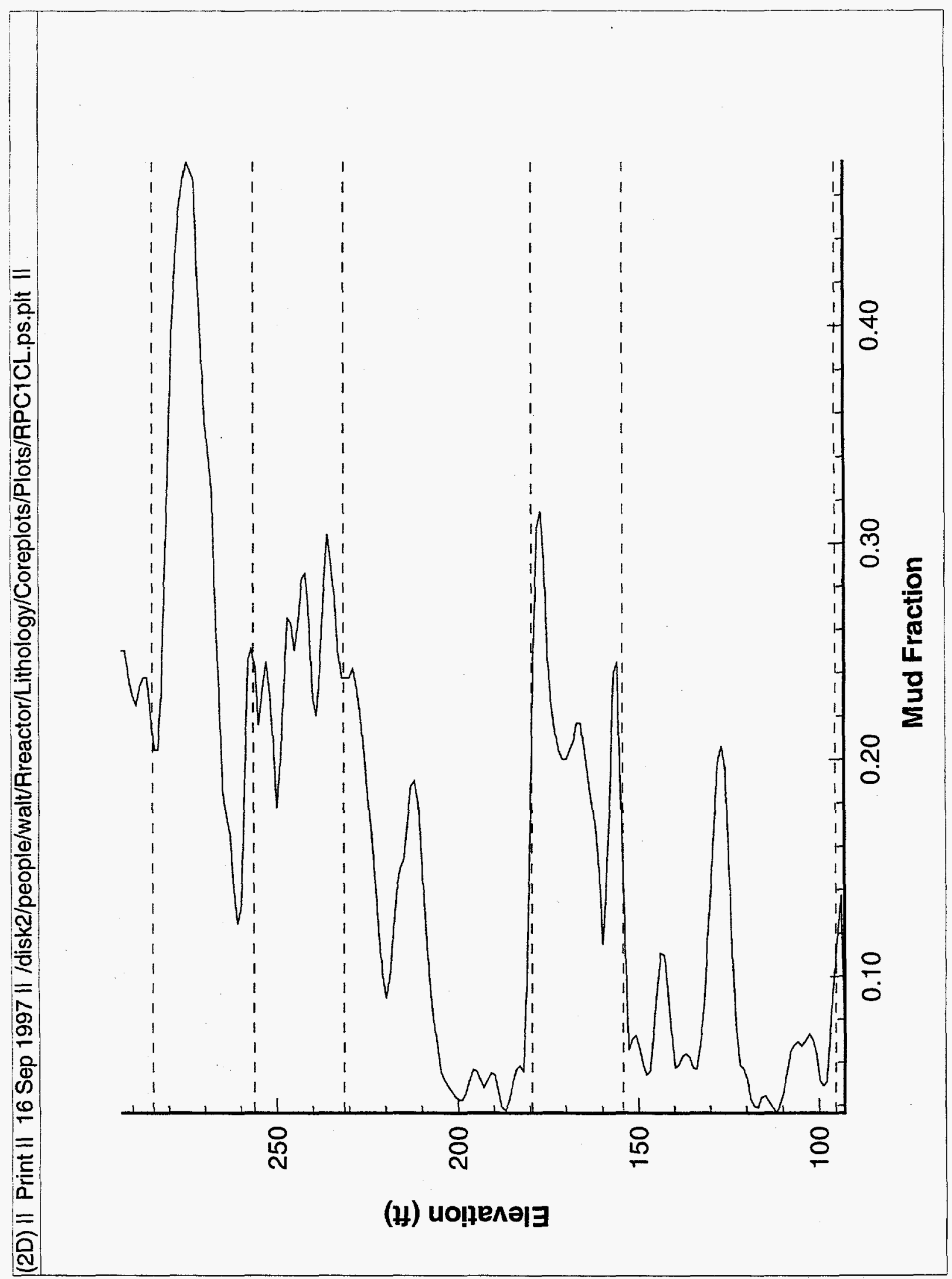




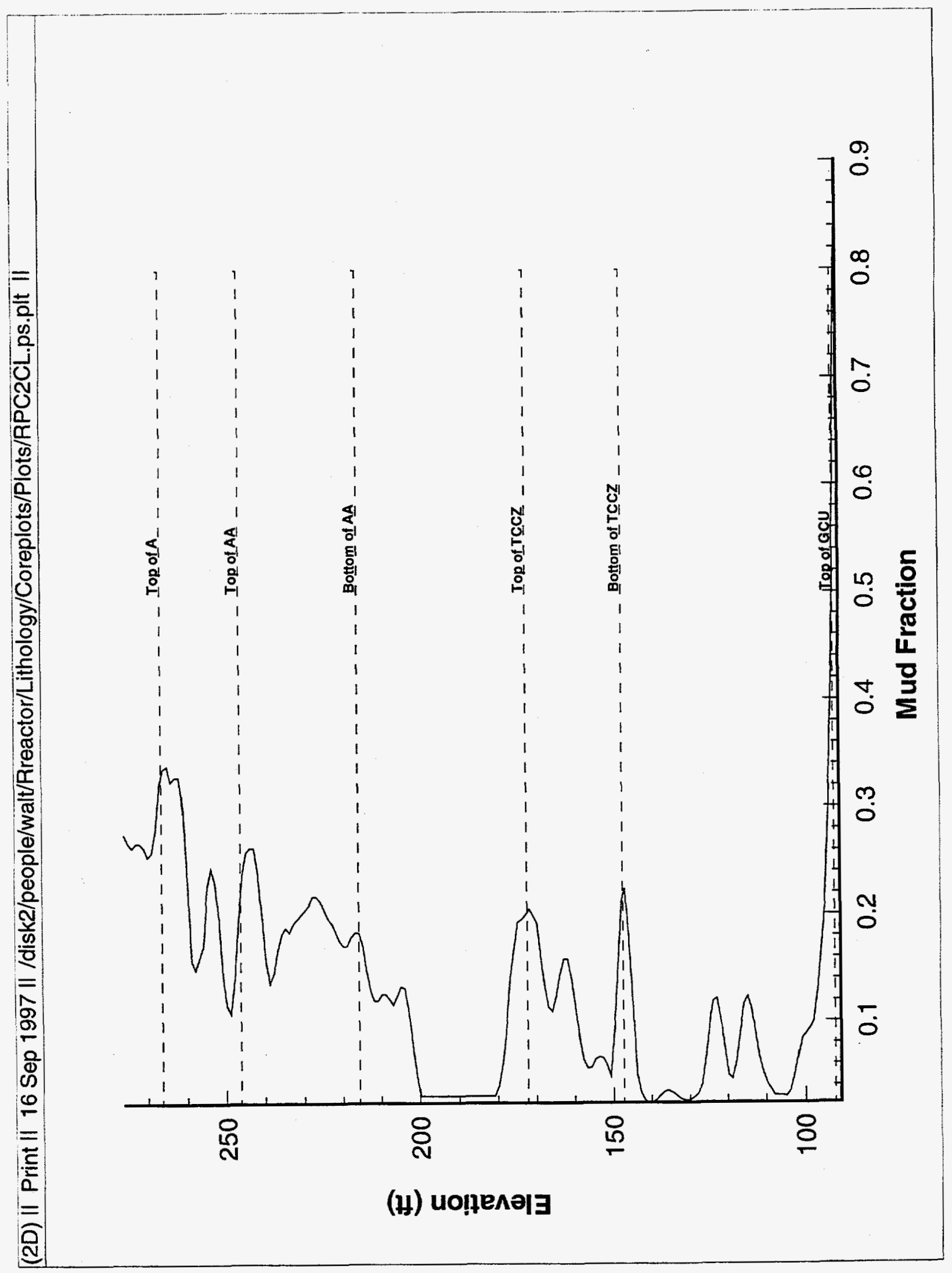




\section{Appendix D \\ Laboratory Data}




\section{Laboratory Permeability Data}

\begin{tabular}{|c|c|c|c|}
\hline \multirow[b]{2}{*}{$\begin{array}{l}\text { Boring and } \\
\text { Horizon ID }\end{array}$} & \multicolumn{3}{|c|}{ Geotechnical Samples (Shelby Tubes) } \\
\hline & $\begin{array}{c}\text { Sample } \\
\text { Depth } \\
\text { (ft, bls) }\end{array}$ & $\begin{array}{l}\text { Horizontal } \\
\text { Permeability } \\
(\mathrm{cm} / \mathrm{sec})\end{array}$ & $\begin{array}{c}\text { Vertical } \\
\text { Permeability } \\
(\mathrm{cm} / \mathrm{sec})\end{array}$ \\
\hline \multicolumn{4}{|l|}{ RCH-1 } \\
\hline Horizon "A" & \multirow{4}{*}{$\begin{array}{c}5-7 \\
10-12 \\
20-22 \\
25-27 \\
\end{array}$} & \multirow{4}{*}{$\begin{array}{c}\text { NA } \\
3.30 \mathrm{E}-06 \\
\text { NA } \\
\text { NA }\end{array}$} & \multirow{4}{*}{$\begin{array}{l}9.90 \mathrm{E}-07 \\
1.40 \mathrm{E}-06 \\
4.70 \mathrm{E}-07 \\
4.40 \mathrm{E}-06\end{array}$} \\
\hline & & & \\
\hline & & & \\
\hline & & & \\
\hline Horizon "AA" & $54-56$ & $2.30 \mathrm{E}-04$ & $1.60 \mathrm{E}-05$ \\
\hline "Tan Clay" confining zone & $125-127$ & NA & $1.50 \mathrm{E}-06$ \\
\hline \multicolumn{4}{|l|}{ RPC-1 } \\
\hline Horizon "A" & $30-30.5$ & NA & $1.10 \mathrm{E}-06$ \\
\hline \multirow{2}{*}{ Transmissive zone } & \multirow{2}{*}{$\begin{array}{l}76-76.5 \\
76.5-77 \\
\end{array}$} & \multirow{2}{*}{$\begin{array}{l}\text { NA } \\
\text { NA }\end{array}$} & \multirow{2}{*}{$\begin{array}{l}7.80 \mathrm{E}-07 \\
1.40 \mathrm{E}-06 \\
\end{array}$} \\
\hline & & & \\
\hline \multirow[t]{2}{*}{ "Tan Clay" confining zone } & \multirow{2}{*}{$\begin{array}{c}127-127.5 \\
127.5-128 \\
145-147 \\
\end{array}$} & \multirow{2}{*}{$\begin{array}{l}\text { NA } \\
\text { NA } \\
\text { NA } \\
\end{array}$} & \multirow{2}{*}{$\begin{array}{l}4.00 \mathrm{E}-04 \\
7.60 \mathrm{E}-05 \\
6.50 \mathrm{E}-07 \\
\end{array}$} \\
\hline & & & \\
\hline Gordon Confining Unit & $211.5-213.5$ & NA & $1.40 \mathrm{E}-07$ \\
\hline \multicolumn{4}{|l|}{$\mathbf{R P C}-\mathbf{2}$} \\
\hline \multirow[t]{2}{*}{ Surface Soils } & \multirow{2}{*}{$\begin{array}{c}8-9 \\
13-15\end{array}$} & \multirow{2}{*}{$\begin{array}{c}\mathrm{NA} \\
1.30 \mathrm{E}-08\end{array}$} & \multirow{2}{*}{$\begin{array}{l}3.50 \mathrm{E}-06 \\
2.90 \mathrm{E}-08 \\
\end{array}$} \\
\hline & & & \\
\hline Horizon "A" & $34-36$ & $5.20 \mathrm{E}-06$ & $8.00 \mathrm{E}-07$ \\
\hline \multicolumn{4}{|l|}{ RPT-6 } \\
\hline \multirow[t]{3}{*}{ Transmissive zone } & \multirow{3}{*}{$\begin{array}{l}71-71.5 \\
71.5-72 \\
80.5-81 \\
\end{array}$} & \multirow{3}{*}{$\begin{array}{l}\text { NA } \\
\text { NA } \\
\text { NA } \\
\end{array}$} & \multirow{3}{*}{$\begin{array}{l}1.20 \mathrm{E}-06 \\
5.40 \mathrm{E}-06 \\
6.10 \mathrm{E}-04\end{array}$} \\
\hline & & & \\
\hline & & & \\
\hline \multirow{3}{*}{ "Tan Clay" confining zone } & \multirow{3}{*}{$\begin{array}{l}116-116.5 \\
116.5-117 \\
128-128.5 \\
\end{array}$} & \multirow{3}{*}{$\begin{array}{l}\text { NA } \\
\text { NA } \\
\text { NA } \\
\end{array}$} & \multirow{3}{*}{$\begin{array}{l}2.20 \mathrm{E}-08 \\
4.40 \mathrm{E}-08 \\
1.50 \mathrm{E}-03 \\
\end{array}$} \\
\hline & & & \\
\hline & & & \\
\hline \multicolumn{4}{|l|}{$\mathbf{P}-\mathbf{2 0}$} \\
\hline Transmissive zone & $105-107$ & $3.40 \mathrm{E}-08$ & $1.10 \mathrm{E}-07$ \\
\hline Gordon Confining Unit & $210-211$ & $9.10 \mathrm{E}-07$ & $1.10 \mathrm{E}-06$ \\
\hline
\end{tabular}

NA - not analyzed 
RBW2CL Minipermeability Tests (E71795,N58712)

\begin{tabular}{|c|c|}
\hline $\begin{array}{c}\text { Elevation } \\
\text { (ft) }\end{array}$ & $\begin{array}{l}\text { Conductivity } \\
\text { (ft/d) }\end{array}$ \\
\hline 164.8 & 7.9 \\
\hline 163.6 & 10.5 \\
\hline 158.8 & 14.6 \\
\hline 157.8 & 12.0 \\
\hline 149.7 & 38.5 \\
\hline 148.6 & 43.9 \\
\hline 143.1 & 34.1 \\
\hline 139.8 & 48.4 \\
\hline 139.1 & 37.9 \\
\hline 137.5 & 27.2 \\
\hline 137.1 & 51.2 \\
\hline 135.1 & 24.6 \\
\hline 134.1 & 0.4 \\
\hline 132.3 & 1.9 \\
\hline 131.6 & 0.7 \\
\hline 129.7 & 1.7 \\
\hline 127.8 & 1.8 \\
\hline 127.1 & 1.6 \\
\hline 125.1 & 2.3 \\
\hline 124.1 & 4.4 \\
\hline 122.8 & 3.3 \\
\hline 122.1 & 6.5 \\
\hline 121.1 & 10.9 \\
\hline 120.1 & 6.2 \\
\hline 118.9 & 1.2 \\
\hline 118.1 & 1.4 \\
\hline 117.1 & 2.0 \\
\hline 115.8 & 1.3 \\
\hline 114.9 & 4.1 \\
\hline 114.1 & 2.1 \\
\hline 113.1 & 1.3 \\
\hline 111.8 & 3.5 \\
\hline 111.1 & 2.2 \\
\hline 110.1 & 0.8 \\
\hline 109.1 & 0.4 \\
\hline 104.5 & 10.1 \\
\hline 103.8 & 111.4 \\
\hline 102.8 & 0.2 \\
\hline 100.1 & 4.8 \\
\hline 99.1 & 10.8 \\
\hline 97.8 & 12.7 \\
\hline 96.7 & 10.2 \\
\hline 95.1 & 6.9 \\
\hline 93.9 & 1.9 \\
\hline 93.1 & 3.6 \\
\hline 89.9 & 3.1 \\
\hline 82.8 & 65.3 \\
\hline 79.1 & 53.4 \\
\hline 78.1 & 47.6 \\
\hline 77.1 & 53.2 \\
\hline
\end{tabular}


RBW2CU Minipermeability Tests (E71786,N58715)

\begin{tabular}{|c|c|}
\hline $\begin{array}{c}\text { Elevation } \\
\text { (ft) }\end{array}$ & $\begin{array}{c}\text { Conductivity } \\
\text { (ft/d) }\end{array}$ \\
\hline 204.9 & $\overline{7.7}$ \\
\hline 204.2 & 15.2 \\
\hline 203.2 & 11.2 \\
\hline 199.8 & 13.6 \\
\hline 199.2 & 14.7 \\
\hline 197.8 & 20.5 \\
\hline 196.9 & 26.0 \\
\hline 196.2 & 40.3 \\
\hline 194.8 & 26.0 \\
\hline 194.2 & 30.6 \\
\hline 190.2 & 39.8 \\
\hline 185.0 & 11.0 \\
\hline 184.2 & 27.9 \\
\hline 183.2 & 33.2 \\
\hline 180.0 & 36.7 \\
\hline 176.8 & 8.9 \\
\hline 175.2 & 13.8 \\
\hline 173.8 & 11.5 \\
\hline 173.2 & 43.3 \\
\hline 167.4 & 7.1 \\
\hline 166.8 & 24.7 \\
\hline 166.2 & 48.0 \\
\hline 164.8 & 11.6 \\
\hline 164.2 & 5.6 \\
\hline 162.7 & 10.5 \\
\hline 162.2 & 26.6 \\
\hline 161.2 & 33.2 \\
\hline 158.5 & 26.9 \\
\hline 157.9 & 23.0 \\
\hline 157.2 & 16.2 \\
\hline 156.2 & 29.7 \\
\hline 155.2 & 52.7 \\
\hline 149.8 & 34.4 \\
\hline 142.9 & 32.2 \\
\hline
\end{tabular}


RCH1 Minipermeability Tests (E74295,N59123)

\begin{tabular}{|c|c|}
\hline $\begin{array}{c}\text { Elevation } \\
\text { (ft) }\end{array}$ & $\begin{array}{c}\text { Conductivity } \\
\text { (ft/d) }\end{array}$ \\
\hline 290.4 & 6.0 \\
\hline 285.3 & 3.6 \\
\hline 280.4 & 13.9 \\
\hline 279.6 & 8.6 \\
\hline 278.3 & 2.5 \\
\hline 275.6 & 11.1 \\
\hline 274.6 & 13.5 \\
\hline 273.6 & 15.3 \\
\hline 272.3 & 9.3 \\
\hline 271.6 & 1.3 \\
\hline 270.6 & 19.5 \\
\hline 269.6 & 19.4 \\
\hline 268.6 & 18.3 \\
\hline 267.6 & 10.1 \\
\hline 266.6 & 11.1 \\
\hline 265.6 & 10.0 \\
\hline 264.3 & 6.5 \\
\hline 263.6 & 4.4 \\
\hline 262.4 & 0.8 \\
\hline 261.3 & 1.0 \\
\hline 260.6 & 12.5 \\
\hline 257.6 & 1.8 \\
\hline 256.3 & 15.2 \\
\hline 255.2 & 20.4 \\
\hline 254.6 & 5.6 \\
\hline 252.3 & 19.4 \\
\hline 251.6 & 2.7 \\
\hline 250.3 & 5.1 \\
\hline 246.6 & 2.9 \\
\hline 245.4 & 1.0 \\
\hline 244.6 & 12.3 \\
\hline 243.4 & 11.2 \\
\hline 242.6 & 28.7 \\
\hline 241.6 & 41.1 \\
\hline 240.3 & 26.7 \\
\hline 239.6 & 35.7 \\
\hline 237.4 & 29.0 \\
\hline 236.6 & 31.5 \\
\hline 235.6 & 24.6 \\
\hline 234.6 & 17.7 \\
\hline 233.6 & 15.4 \\
\hline 232.1 & 5.4 \\
\hline 231.2 & 10.7 \\
\hline 230.6 & 4.8 \\
\hline 229.3 & 19.1 \\
\hline 228.3 & 11.6 \\
\hline 227.3 & 21.3 \\
\hline 226.3 & 16.3 \\
\hline 225.5 & 22.3 \\
\hline 222.6 & 45.0 \\
\hline
\end{tabular}


RCH1 Minipermeability Tests (E74295,N59123)

\begin{tabular}{|c|c|}
\hline $\begin{array}{c}\text { Elevation } \\
\text { (ft) }\end{array}$ & $\begin{array}{c}\text { Conductivity } \\
\text { (ft/d) }\end{array}$ \\
\hline 221.6 & 27.0 \\
\hline 220.6 & 26.2 \\
\hline 219.6 & 27.0 \\
\hline 217.6 & 30.9 \\
\hline 216.6 & 30.7 \\
\hline 215.6 & 46.5 \\
\hline 214.6 & 90.0 \\
\hline 213.6 & 91.5 \\
\hline 211.6 & 121.5 \\
\hline 207.6 & 111.3 \\
\hline 206.6 & 100.2 \\
\hline 205.6 & 49.0 \\
\hline 201.4 & 31.8 \\
\hline 200.6 & 28.0 \\
\hline 199.6 & 62.2 \\
\hline 198.6 & 62.4 \\
\hline 197.6 & 51.8 \\
\hline 196.6 & 43.5 \\
\hline 195.6 & 64.0 \\
\hline 194.6 & 64.7 \\
\hline 192.6 & 77.0 \\
\hline 191.6 & 28.2 \\
\hline 190.6 & 92.3 \\
\hline 189.6 & 77.6 \\
\hline 188.6 & 67.3 \\
\hline 187.6 & 102.0 \\
\hline 186.6 & 27.8 \\
\hline 185.6 & 75.9 \\
\hline 184.6 & 87.9 \\
\hline 183.6 & 83.1 \\
\hline 182.4 & 75.7 \\
\hline 181.6 & 74.6 \\
\hline 180.6 & 57.0 \\
\hline 179.6 & 34.5 \\
\hline 175.0 & 27.2 \\
\hline 174.4 & 55.5 \\
\hline 173.0 & 36.2 \\
\hline 172.6 & 92.1 \\
\hline 171.3 & 64.5 \\
\hline 170.6 & 99.6 \\
\hline 169.6 & 112.1 \\
\hline 168.6 & 73.8 \\
\hline 167.6 & 92.2 \\
\hline 166.6 & 113.3 \\
\hline 164.6 & 84.6 \\
\hline 162.6 & 63.0 \\
\hline 161.6 & 107.1 \\
\hline 160.6 & 94.3 \\
\hline 159.6 & 135.3 \\
\hline 157.6 & 48.2 \\
\hline
\end{tabular}


RCH1 Minipermeability Tests (E74295,N59123)

\begin{tabular}{|c||c|}
\hline $\begin{array}{c}\text { Elevation } \\
\text { (ft) }\end{array}$ & $\begin{array}{c}\text { Conductivity } \\
\text { (ft/d) }\end{array}$ \\
\hline 156.6 & 51.5 \\
\hline 155.6 & 84.3 \\
\hline 154.0 & 53.8 \\
\hline 153.6 & 50.0 \\
\hline 152.2 & 55.4 \\
\hline 106.6 & 2.9 \\
\hline
\end{tabular}


RPC1CL Minipermeability Tests (E74262,N57923)

\begin{tabular}{|c|c|}
\hline $\begin{array}{c}\text { Elevation } \\
\text { (ft) }\end{array}$ & $\begin{array}{c}\text { Conductivity } \\
\text { (ft/d) }\end{array}$ \\
\hline 292.3 & 1.6 \\
\hline 289.9 & 3.3 \\
\hline 287.6 & 0.7 \\
\hline 285.7 & 0.6 \\
\hline 284.3 & 3.4 \\
\hline 283.1 & 3.7 \\
\hline 282.0 & 1.4 \\
\hline 280.5 & 1.0 \\
\hline 264.0 & 0.9 \\
\hline 262.9 & 2.6 \\
\hline 259.3 & 2.5 \\
\hline 258.1 & 3.7 \\
\hline 256.8 & 3.8 \\
\hline 256.3 & 2.7 \\
\hline 254.0 & 3.9 \\
\hline 252.8 & 1.7 \\
\hline 248.0 & 1.6 \\
\hline 247.3 & 1.7 \\
\hline 246.3 & 1.8 \\
\hline 245.3 & 0.7 \\
\hline 243.9 & 0.7 \\
\hline 239.3 & 4.4 \\
\hline 238.3 & 2.8 \\
\hline 237.3 & 1.6 \\
\hline 236.3 & 2.8 \\
\hline 234.9 & 3.7 \\
\hline 234.2 & 3.8 \\
\hline 233.3 & 2.9 \\
\hline 232.3 & 2.2 \\
\hline 230.3 & 2.0 \\
\hline 227.3 & 2.7 \\
\hline 226.0 & 2.0 \\
\hline 225.3 & 13.6 \\
\hline 224.0 & 13.5 \\
\hline 223.3 & 17.2 \\
\hline 222.3 & 18.2 \\
\hline 219.3 & 4.6 \\
\hline 218.3 & 13.1 \\
\hline 217.3 & 11.8 \\
\hline 216.3 & 15.7 \\
\hline 215.3 & 16.6 \\
\hline 214.3 & 12.0 \\
\hline 213.3 & 5.7 \\
\hline 212.3 & 3.6 \\
\hline 211.3 & 8.7 \\
\hline 208.3 & 5.3 \\
\hline 204.3 & 12.3 \\
\hline 197.3 & 9.2 \\
\hline 196.0 & 41.8 \\
\hline 195.3 & 42.9 \\
\hline
\end{tabular}


RPC1CL Minipermeability Tests (E74262,N57923)

\begin{tabular}{|c|c|}
\hline $\begin{array}{l}\text { Elevation } \\
\text { (ft) }\end{array}$ & $\begin{array}{c}\text { Conductivity } \\
\text { (ft/d) }\end{array}$ \\
\hline 184.1 & 43.7 \\
\hline 177.1 & 56.2 \\
\hline 176.3 & 60.3 \\
\hline 175.3 & 47.9 \\
\hline 172.3 & 65.0 \\
\hline 171.3 & 50.1 \\
\hline 170.3 & 42.6 \\
\hline 169.3 & 52.3 \\
\hline 167.8 & 37.6 \\
\hline 166.8 & 7.9 \\
\hline 166.3 & 19.1 \\
\hline 155.1 & 82.6 \\
\hline 150.8 & 50.4 \\
\hline 149.0 & 29.1 \\
\hline 148.1 & 62.8 \\
\hline 146.0 & 20.1 \\
\hline 143.8 & 47.8 \\
\hline 143.1 & 19.1 \\
\hline 141.8 & 32.0 \\
\hline 141.3 & 35.7 \\
\hline 140.3 & 47.1 \\
\hline 139.0 & 34.8 \\
\hline 138.3 & 39.9 \\
\hline
\end{tabular}


RPC2CL Minipermeability Tests (E76121,N58077)

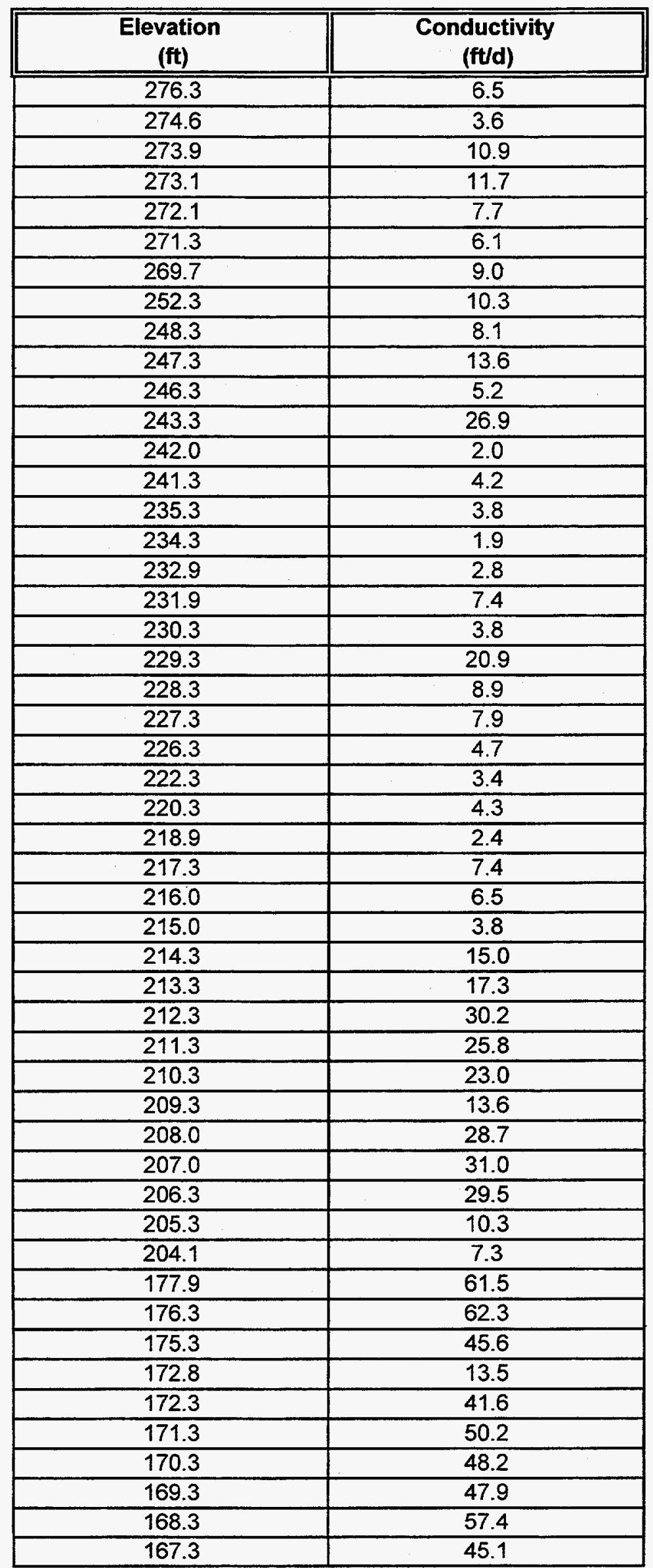


RPC2CL Minipermeability Tests (E76121,N58077)

\begin{tabular}{|c|c|}
\hline $\begin{array}{c}\begin{array}{c}\text { Elevation } \\
\text { (ft) }\end{array} \\
\end{array}$ & $\begin{array}{c}\text { Conductivity } \\
\text { (ft/d) }\end{array}$ \\
\hline 166.3 & 65.7 \\
\hline 165.3 & 69.0 \\
\hline 164.3 & 60.3 \\
\hline 162.7 & 30.2 \\
\hline 162.3 & 13.8 \\
\hline 161.3 & 32.2 \\
\hline 158.3 & 46.5 \\
\hline 157.3 & 53.0 \\
\hline 156.3 & 57.0 \\
\hline 155.3 & 48.2 \\
\hline 154.3 & 52.3 \\
\hline 153.0 & 57.9 \\
\hline 152.3 & 57.9 \\
\hline 147.3 & 50.8 \\
\hline 146.3 & 65.3 \\
\hline 145.3 & 57.4 \\
\hline 144.3 & 58.3 \\
\hline 143.1 & 53.6 \\
\hline 142.3 & 54.9 \\
\hline 141.3 & 50.7 \\
\hline 140.3 & 52.7 \\
\hline 139.3 & 65.9 \\
\hline 138.0 & 104.5 \\
\hline 137.3 & 81.8 \\
\hline 136.3 & 103.9 \\
\hline 133.3 & 83.0 \\
\hline 132.3 & 78.0 \\
\hline 131.3 & 79.5 \\
\hline 130.0 & 78.4 \\
\hline 129.3 & 54.7 \\
\hline 128.3 & 83.9 \\
\hline 127.3 & 58.6 \\
\hline 126.3 & 52.5 \\
\hline 125.3 & 31.3 \\
\hline 122.8 & 20.4 \\
\hline 121.3 & 22.1 \\
\hline 120.3 & 33.1 \\
\hline 118.3 & 27.0 \\
\hline 117.3 & 27.8 \\
\hline 115.8 & 17.2 \\
\hline 115.3 & 22.5 \\
\hline 113.3 & 47.8 \\
\hline 112.3 & 38.8 \\
\hline 103.3 & 17.7 \\
\hline 102.3 & 24.8 \\
\hline
\end{tabular}




\section{Appendix E \\ Hydrostratigraphic Data}


R-Reactor Area Data

\begin{tabular}{|c|c|c|c|c|c|c|c|c|c|c|}
\hline Well ID & $\begin{array}{c}\text { SRS } \\
\text { Northing (ft) }\end{array}$ & $\begin{array}{c}\text { SRS } \\
\text { Easting (it) }\end{array}$ & $\begin{array}{l}\text { Ground } \\
\text { Surtace (it) }\end{array}$ & $\begin{array}{l}\text { Top of 'A' Horizon } \\
\text { above msl (ft) }\end{array}$ & $\begin{array}{l}\text { Top of 'AA' Horizon } \\
\text { above msi (tt) }\end{array}$ & $\begin{array}{c}\text { Bottom of 'AA' Horlzon } \\
\text { above msi (fi) }\end{array}$ & $\begin{array}{l}\text { Top of Tan Clay } \\
\text { Confining Zone (ft) }\end{array}$ & $\begin{array}{l}\text { Bottom of Tan Clay } \\
\text { Confining Zone. (it) }\end{array}$ & $\begin{array}{l}\text { Top of Gordon } \\
\text { Confining Unit (tt) }\end{array}$ & $\begin{array}{l}\text { Bottom of Gordon } \\
\text { Confining Unlt (tt) }\end{array}$ \\
\hline$\overline{\mathrm{RPC}-01 \mathrm{CL}}$ & 57923.3 & 74261.9 & 304.3 & 284.3 & 256.3 & 231.3 & 179.3 & 154.3 & 95.3 & ND \\
\hline RPC-02CL & 58077.2 & 76120.7 & 292.3 & 266.3 & 246.3 & 215.8 & 172.3 & 147.3 & 92.3 & ND \\
\hline RBW-2CL & 58712.0 & 71795.1 & 325.1 & 325.1 & 295.1 & 225.1 & 190.1 & 169.1 & 90.12 & ND \\
\hline $\mathrm{RCH}-001$ & 59123.4 & 74294.9 & 302.6 & 299.6 & 272.6 & 245.6 & 202.6 & 175.6 & 105.6 & ND \\
\hline $\mathrm{N}-13$ & 66632.1 & 70856.2 & 335.9 & 328.9 & 318.9 & 262.9 & 235.9 & 223.9 & 115.9 & 103.9 \\
\hline $\mathrm{P}-20$ & 56075.2 & 76784.5 & 287.7 & 277.7 & 257.7 & 215.2 & 171.7 & 152.0 & 81.7 & 73.7 \\
\hline RCP-1A & 56968.1 & 74238.3 & 294.8 & 289.8 & 263.8 & 221.8 & 182.8 & 161.8 & 83.8 & 77.8 \\
\hline RBW1 & 62042.5 & 74232.0 & 284.0 & 284.0 & 268.5 & 234.0 & 221.0 & 214.0 & ND & ND \\
\hline RPT1 & 55946.9 & 75568.7 & 290.3 & 270.3 & 238.3 & 214.3 & 178.3 & ND & ND & $\mathrm{ND}$ \\
\hline RPT2 & 56881.7 & 76333.4 & 284.3 & 263.3 & 244.3 & 220.3 & 194.3 & 164.3 & ND & ND \\
\hline RPT3 & 57479.8 & 75939.7 & 298.3 & 275.3 & 228.3 & 203.3 & 175.3 & 155.3 & ND & $\mathrm{ND}$ \\
\hline RPT5 & 58755.7 & 76279.4 & 294.5 & 261.5 & 230.5 & 204.5 & 179.5 & 148.5 & ND & ND \\
\hline RPT6 & 59311.2 & 75363.4 & 297.9 & 293.9 & 263.9 & 240.9 & 190.9 & 167.9 & ND & ND \\
\hline RPT7 & 57848.3 & 75014.3 & 308.5 & 270.5 & 242.5 & 213.5 & 176.5 & 152.5 & ND & ND \\
\hline RPT8 & 58206.4 & 75404.3 & 307.9 & 280.9 & 242.9 & 217.9 & 177.9 & 155.9 & 98.9 & $\mathrm{ND}$ \\
\hline RPT9 & 58625.3 & 75268.8 & 301.7 & 269.7 & 237.7 & 211.7 & 173.7 & 155.7 & 102.7 & ND \\
\hline
\end{tabular}

ND - No Data available 


\section{Appendix F \\ Conductivity Inferred from Core, Laboratory Permeability, and CPT Data}


Conductivity Data at N13

\begin{tabular}{|c|c|c|c|}
\hline $\begin{array}{c}\text { Elevation } \\
\text { (ft) }\end{array}$ & $\begin{array}{c}\text { Kh } \\
\text { (ft/day) }\end{array}$ & $\begin{array}{c}\text { Kv } \\
\text { (ft/day) }\end{array}$ & $\begin{array}{c}\text { Data } \\
\text { Source }\end{array}$ \\
\hline 320.4 & $1.13 \mathrm{E}-01$ & $3.13 \mathrm{E}-02$ & core \\
\hline 319.4 & $1.13 \mathrm{E}-01$ & $3.13 \mathrm{E}-02$ & core \\
\hline 318.4 & $7.24 \mathrm{E}+00$ & $2.01 \mathrm{E}+00$ & core \\
\hline 317.4 & $1.15 \mathrm{E}+01$ & $3.19 \mathrm{E}+00$ & core \\
\hline 315.4 & $1.83 \mathrm{E}+01$ & $5.07 \mathrm{E}+00$ & core \\
\hline 314.4 & $1.83 \mathrm{E}+01$ & $5.07 \mathrm{E}+00$ & core \\
\hline 313.4 & $1.83 \mathrm{E}+01$ & $5.07 \mathrm{E}+00$ & core \\
\hline 312.4 & $1.83 \mathrm{E}+01$ & $5.07 \mathrm{E}+00$ & core \\
\hline 311.4 & $1.83 \mathrm{E}+01$ & $5.07 \mathrm{E}+00$ & core \\
\hline 310.4 & $1.83 \mathrm{E}+01$ & $5.07 \mathrm{E}+00$ & core \\
\hline 309.4 & $2.90 \mathrm{E}+01$ & $8.05 \mathrm{E}+00$ & core \\
\hline 308.4 & $2.90 \mathrm{E}+01$ & $8.05 \mathrm{E}+00$ & core \\
\hline 307.4 & $1.83 \mathrm{E}+01$ & $5.07 \mathrm{E}+00$ & core \\
\hline 306.4 & $1.15 \mathrm{E}+01$ & $3.19 \mathrm{E}+00$ & core \\
\hline 305.4 & $1.83 \mathrm{E}+01$ & $5.07 \mathrm{E}+00$ & core \\
\hline 304.4 & $1.83 \mathrm{E}+01$ & $5.07 \mathrm{E}+00$ & core \\
\hline 303.4 & $1.83 \mathrm{E}+01$ & $5.07 \mathrm{E}+00$ & core \\
\hline 302.4 & $1.83 \mathrm{E}+01$ & $5.07 \mathrm{E}+00$ & core \\
\hline 301.4 & $1.83 \mathrm{E}+01$ & $5.07 \mathrm{E}+00$ & core \\
\hline 300.4 & $1.83 \mathrm{E}+01$ & $5.07 \mathrm{E}+00$ & core \\
\hline 299.4 & $1.83 \mathrm{E}+01$ & $5.07 \mathrm{E}+00$ & core \\
\hline 298.4 & $1.15 \mathrm{E}+01$ & $3.19 \mathrm{E}+00$ & core \\
\hline 297.4 & $1.83 \mathrm{E}+01$ & $5.07 \mathrm{E}+00$ & core \\
\hline 296.4 & $1.83 \mathrm{E}+01$ & $5.07 \mathrm{E}+00$ & core \\
\hline 295.4 & $2.90 \mathrm{E}+01$ & $8.05 \mathrm{E}+00$ & core \\
\hline 294.4 & $2.90 \mathrm{E}+01$ & $8.05 \mathrm{E}+00$ & core \\
\hline 293.4 & $1.83 \mathrm{E}+01$ & $5.07 \mathrm{E}+00$ & core \\
\hline 292.4 & $2.90 \mathrm{E}+01$ & $8.05 \mathrm{E}+00$ & core \\
\hline 291.4 & $2.90 \mathrm{E}+01$ & $8.05 \mathrm{E}+00$ & core \\
\hline 290.4 & $2.90 \mathrm{E}+01$ & $8.05 \mathrm{E}+00$ & core \\
\hline 289.4 & $2.90 \mathrm{E}+01$ & $8.05 \mathrm{E}+00$ & core \\
\hline 287.4 & $3.83 \mathrm{E}+01$ & $1.06 \mathrm{E}+01$ & core \\
\hline 286.4 & $3.83 \mathrm{E}+01$ & $1.06 \mathrm{E}+01$ & core \\
\hline 285.4 & $4.60 \mathrm{E}+01$ & $1.28 \mathrm{E}+01$ & core \\
\hline 284.4 & $5.54 \mathrm{E}+01$ & $1.54 \mathrm{E}+01$ & core \\
\hline 283.4 & $4.60 \mathrm{E}+01$ & $1.28 \mathrm{E}+01$ & core \\
\hline 282.4 & $3.83 \mathrm{E}+01$ & $1.06 \mathrm{E}+01$ & core \\
\hline 281.4 & $4.60 \mathrm{E}+01$ & $1.28 \mathrm{E}+01$ & core \\
\hline 280.4 & $5.54 \mathrm{E}+01$ & $1.54 \mathrm{E}+01$ & core \\
\hline 279.4 & $5.54 \mathrm{E}+01$ & $1.54 \mathrm{E}+01$ & core \\
\hline 278.4 & $5.54 \mathrm{E}+01$ & $1.54 \mathrm{E}+01$ & core \\
\hline 277.4 & $5.54 \mathrm{E}+01$ & $1.54 \mathrm{E}+01$ & core \\
\hline 276.4 & $5.54 \mathrm{E}+01$ & $1.54 \mathrm{E}+01$ & core \\
\hline 275.4 & $6.08 \mathrm{E}+01$ & $1.69 \mathrm{E}+01$ & core \\
\hline 27.4 & $6.08 \mathrm{E}+01$ & $1.69 \mathrm{E}+01$ & core \\
\hline $1.83 \mathrm{E}+01$ & $5.07 \mathrm{E}+00$ & core \\
\hline 3.4 & $3.83 \mathrm{E}+01$ & $1.06 \mathrm{E}+01$ & core \\
\hline
\end{tabular}


Conductivity Data at N13

\begin{tabular}{|c|c|c|c|}
\hline $\begin{array}{l}\text { Elevation } \\
\text { (ft) }\end{array}$ & $\begin{array}{c}\text { Kh } \\
\text { (ft/day) }\end{array}$ & $\begin{array}{c}\overline{K v} \\
\text { (ft/day) }\end{array}$ & $\begin{array}{c}\text { Data } \\
\text { Source }\end{array}$ \\
\hline 270.4 & $4.60 E+01$ & $1.28 \mathrm{E}+01$ & core \\
\hline 269.4 & $2.90 E+01$ & $8.05 \mathrm{E}+00$ & core \\
\hline 268.4 & $2.90 E+01$ & $8.05 E+00$ & core \\
\hline 267.4 & $4.60 E+01$ & $1.28 \mathrm{E}+01$ & core \\
\hline 266.4 & $3.83 E+01$ & $1.06 \mathrm{E}+01$ & core \\
\hline 265.4 & $5.54 \mathrm{E}+01$ & $1.54 \mathrm{E}+01$ & core \\
\hline 264.4 & $4.56 \mathrm{E}+00$ & $1.27 \mathrm{E}+00$ & core \\
\hline 263.4 & $4.56 \mathrm{E}+00$ & $1.27 \mathrm{E}+00$ & core \\
\hline 262.4 & $2.54 \mathrm{E}-03$ & $7.05 E-04$ & core \\
\hline 261.4 & $1.82 E+01$ & $5.06 \mathrm{E}+00$ & core \\
\hline 260.4 & $1.82 E+01$ & $5.06 \mathrm{E}+00$ & core \\
\hline 259.4 & $1.39 E-01$ & $3.86 E-02$ & core \\
\hline 258.4 & $1.39 E-01$ & $3.86 E-02$ & core \\
\hline 257.4 & $1.82 E+01$ & $5.06 \mathrm{E}+00$ & core \\
\hline 256.4 & $1.82 E+01$ & $5.06 E+00$ & core \\
\hline 255.4 & $1.50 \mathrm{E}-03$ & $4.18 E-04$ & core \\
\hline 254.4 & $2.89 E+01$ & $8.04 E+00$ & core \\
\hline 253.4 & $1.82 E+01$ & $5.06 \mathrm{E}+00$ & core \\
\hline 252.4 & $3.82 E+01$ & $1.06 \mathrm{E}+01$ & core \\
\hline 251.4 & $3.82 E+01$ & $1.06 \mathrm{E}+01$ & core \\
\hline 250.4 & $3.82 E+01$ & $1.06 \mathrm{E}+01$ & core \\
\hline 249.4 & $1.82 E+01$ & $5.06 \mathrm{E}+00$ & core \\
\hline 248.4 & $1.82 E+01$ & $5.06 \mathrm{E}+00$ & core \\
\hline 247.4 & $2.89 \mathrm{E}+01$ & $8.04 \mathrm{E}+00$ & core \\
\hline 246.4 & $2.89 E+01$ & $8.04 E+00$ & core \\
\hline 245.4 & $1.82 \mathrm{E}+01$ & $5.06 \mathrm{E}+00$ & core \\
\hline 244.4 & $1.50 \mathrm{E}-03$ & $4.18 E-04$ & core \\
\hline 243.4 & 8.91E-04 & $2.48 \mathrm{E}-04$ & core \\
\hline 242.4 & $1.82 \mathrm{E}+01$ & $5.06 \mathrm{E}+00$ & core \\
\hline 241.4 & $4.60 \mathrm{E}+01$ & $1.28 \mathrm{E}+01$ & core \\
\hline 240.4 & $1.50 \mathrm{E}-03$ & $4.18 \mathrm{E}-04$ & core \\
\hline 239.4 & $2.54 E-03$ & $7.05 E-04$ & core \\
\hline 238.4 & $1.82 E+01$ & $5.06 \mathrm{E}+00$ & core \\
\hline 237.4 & $2.89 E+01$ & $8.04 E+00$ & core \\
\hline 236.4 & $5.54 E+01$ & $1.54 \mathrm{E}+01$ & core \\
\hline 235.4 & $6.07 E+01$ & $1.69 \mathrm{E}+01$ & core \\
\hline 234.4 & 8.91E-04 & $2.48 E-04$ & core \\
\hline 233.4 & $1.82 E+01$ & $5.06 \mathrm{E}+00$ & core \\
\hline 232.4 & $2.89 E+01$ & $8.04 E+00$ & core \\
\hline 231.4 & $1.50 \mathrm{E}-03$ & $4.18 E-04$ & core \\
\hline 230.4 & $8.36 E-05$ & $2.32 E-05$ & core \\
\hline 229.4 & $3.20 E-05$ & $8.90 E-06$ & core \\
\hline 228.4 & 1.15E-04 & $3.20 \mathrm{E}-05$ & core \\
\hline 227.4 & $3.20 E-05$ & $8.90 E-06$ & core \\
\hline 226.4 & $2.43 E-05$ & 6.74E-06 & core \\
\hline 225.4 & $1.50 \mathrm{E}-03$ & $4.18 E-04$ & core \\
\hline 224.4 & $2.54 E-03$ & $7.05 E-04$ & core \\
\hline 223.4 & $1.82 \mathrm{E}+01$ & $5.07 E+00$ & core \\
\hline
\end{tabular}


Conductivity Data at N13

\begin{tabular}{|c|c|c|c|}
\hline $\begin{array}{c}\text { Elevation } \\
\text { (ft) }\end{array}$ & $\begin{array}{c}\text { Kh } \\
\text { (ft/day) }\end{array}$ & $\begin{array}{c}\mathrm{Kv} \\
\text { (ft/day) }\end{array}$ & $\begin{array}{l}\text { Data } \\
\text { Source }\end{array}$ \\
\hline 222.4 & $1.69 \mathrm{E}-04$ & $4.69 \mathrm{E}-05$ & core \\
\hline 221.4 & $2.10 E+01$ & $5.82 E+00$ & core \\
\hline 220.4 & $2.90 E+01$ & $8.05 E+00$ & core \\
\hline 219.4 & $2.20 \mathrm{E}+01$ & $6.10 E+00$ & core \\
\hline 218.4 & $2.14 E+01$ & $5.93 E+00$ & core \\
\hline 217.4 & $7.93 E+00$ & $2.20 E+00$ & core \\
\hline 216.4 & $4.15 E+00$ & $1.15 \mathrm{E}+00$ & core \\
\hline 215.4 & $5.74 E+00$ & $1.59 \mathrm{E}+00$ & core \\
\hline 214.4 & $4.15 E+00$ & $1.15 \mathrm{E}+00$ & core \\
\hline 213.4 & $1.59 E+01$ & $4.41 E+00$ & core \\
\hline 212.4 & $2.10 E+01$ & $5.82 E+00$ & core \\
\hline 211.4 & $5.59 E+01$ & $1.55 \mathrm{E}+01$ & core \\
\hline 210.4 & $2.41 \mathrm{E}+01$ & $6.69 E+00$ & core \\
\hline 209.4 & $2.04 \mathrm{E}+01$ & $5.66 E+00$ & core \\
\hline 208.4 & $1.38 \mathrm{E}+01$ & $3.84 \mathrm{E}+00$ & core \\
\hline 207.4 & $1.38 \mathrm{E}+01$ & $3.84 \mathrm{E}+00$ & core \\
\hline 206.4 & $1.38 E+01$ & $3.84 \mathrm{E}+00$ & core \\
\hline 205.4 & $2.52 E+01$ & $7.01 \mathrm{E}+00$ & core \\
\hline 204.4 & $2.20 E+01$ & $6.10 \mathrm{E}+00$ & core \\
\hline 203.4 & $1.16 E+01$ & $3.22 \mathrm{E}+00$ & core \\
\hline 202.4 & $1.41 E+01$ & $3.91 \mathrm{E}+00$ & core \\
\hline 201.4 & $2.50 \mathrm{E}+01$ & $6.94 \mathrm{E}+00$ & core \\
\hline 200.4 & $2.30 E+01$ & $6.39 \mathrm{E}+00$ & core \\
\hline 199.4 & $2.50 E+01$ & $6.94 E+00$ & core \\
\hline 198.4 & $1.62 E+01$ & $4.49 E+00$ & core \\
\hline 197.4 & $1.62 E+01$ & $4.49 E+00$ & core \\
\hline 196.4 & $2.10 \mathrm{E}+01$ & $5.82 E+00$ & core \\
\hline 195.4 & $1.45 E+01$ & $4.02 E+00$ & core \\
\hline 194.4 & $1.45 E+01$ & $4.02 E+00$ & core \\
\hline 193.4 & $1.10 E+01$ & $3.05 E+00$ & core \\
\hline 192.4 & $1.62 E+01$ & $4.49 E+00$ & core \\
\hline 191.4 & $1.52 \mathrm{E}+01$ & $4.21 E+00$ & core \\
\hline 190.4 & $2.30 \mathrm{E}+01$ & $6.39 E+00$ & core \\
\hline 189.4 & $2.30 E+01$ & $6.39 E+00$ & core \\
\hline 188.4 & $1.38 E+01$ & $3.84 E+00$ & core \\
\hline 187.4 & $7.93 E+00$ & $2.20 \mathrm{E}+00$ & core \\
\hline 186.4 & $9.54 E+00$ & $2.65 E+00$ & core \\
\hline 185.4 & $1.82 E+01$ & $5.07 E+00$ & core \\
\hline 184.4 & $2.34 \mathrm{E}+01$ & $6.51 \mathrm{E}+00$ & core \\
\hline 183.4 & $2.41 E+01$ & $6.69 E+00$ & core \\
\hline 182.4 & $2.90 E+01$ & $8.05 E+00$ & core \\
\hline 181.4 & $2.90 \mathrm{E}+01$ & $8.05 E+00$ & core \\
\hline 180.4 & $2.90 \mathrm{E}+01$ & $8.05 E+00$ & core \\
\hline 179.4 & $2.00 E+01$ & $5.56 E+00$ & core \\
\hline 178.4 & $1.82 \mathrm{E}+01$ & $5.07 E+00$ & core \\
\hline 177.4 & $1.66 E+01$ & $4.62 E+00$ & core \\
\hline 176.4 & $2.20 \mathrm{E}+01$ & $6.10 \mathrm{E}+00$ & core \\
\hline 175.4 & $1.21 \mathrm{E}-03$ & 3.37E-04 & core \\
\hline
\end{tabular}


Conductivity Data at N13

\begin{tabular}{|c|c|c|c|}
\hline $\begin{array}{c}\text { Elevation } \\
\text { (ft) }\end{array}$ & $\begin{array}{c}\text { Kh } \\
\text { (ft/day) }\end{array}$ & $\begin{array}{c}\text { Kv } \\
\text { (ft/day) }\end{array}$ & $\begin{array}{c}\text { Data } \\
\text { Source }\end{array}$ \\
\hline 174.4 & $1.74 \mathrm{E}+01$ & $4.84 \mathrm{E}+00$ & core \\
\hline 173.4 & $3.18 \mathrm{E}+01$ & $8.83 \mathrm{E}+00$ & core \\
\hline 172.4 & $1.62 \mathrm{E}+01$ & $4.49 \mathrm{E}+00$ & core \\
\hline 171.4 & $2.41 \mathrm{E}+01$ & $6.69 \mathrm{E}+00$ & core \\
\hline 170.4 & $3.18 \mathrm{E}+01$ & $8.83 \mathrm{E}+00$ & core \\
\hline 169.4 & $3.18 \mathrm{E}+01$ & $8.83 \mathrm{E}+00$ & core \\
\hline 167.4 & $3.09 \mathrm{E}+01$ & $8.59 \mathrm{E}+00$ & core \\
\hline 166.4 & $3.09 \mathrm{E}+01$ & $8.59 \mathrm{E}+00$ & core \\
\hline 165.4 & $3.24 \mathrm{E}+01$ & $8.99 \mathrm{E}+00$ & core \\
\hline 164.4 & $3.18 \mathrm{E}+01$ & $8.83 \mathrm{E}+00$ & core \\
\hline 163.4 & $1.26 \mathrm{E}+01$ & $3.50 \mathrm{E}+00$ & core \\
\hline 162.4 & $1.02 \mathrm{E}+01$ & $2.83 \mathrm{E}+00$ & core \\
\hline 161.4 & $3.09 \mathrm{E}+01$ & $8.59 \mathrm{E}+00$ & core \\
\hline 160.4 & $2.00 \mathrm{E}+01$ & $5.56 \mathrm{E}+00$ & core \\
\hline 159.4 & $3.09 \mathrm{E}+01$ & $8.59 \mathrm{E}+00$ & core \\
\hline 158.4 & $3.09 \mathrm{E}+01$ & $8.59 \mathrm{E}+00$ & core \\
\hline 157.4 & $3.04 \mathrm{E}+01$ & $8.43 \mathrm{E}+00$ & core \\
\hline 156.4 & $3.04 \mathrm{E}+01$ & $8.43 \mathrm{E}+00$ & core \\
\hline 155.4 & $3.04 \mathrm{E}+01$ & $8.43 \mathrm{E}+00$ & core \\
\hline 154.4 & $2.10 \mathrm{E}+01$ & $5.82 \mathrm{E}+00$ & core \\
\hline 153.4 & $2.10 \mathrm{E}+01$ & $5.82 \mathrm{E}+00$ & core \\
\hline 152.4 & $2.90 \mathrm{E}+01$ & $8.05 \mathrm{E}+00$ & core \\
\hline 151.4 & $3.09 \mathrm{E}+01$ & $8.59 \mathrm{E}+00$ & core \\
\hline 150.4 & $3.18 \mathrm{E}+01$ & $8.83 \mathrm{E}+00$ & core \\
\hline 149.4 & $1.52 \mathrm{E}+01$ & $4.21 \mathrm{E}+00$ & core \\
\hline 148.4 & $1.59 \mathrm{E}+01$ & $4.41 \mathrm{E}+00$ & core \\
\hline 147.4 & $2.90 \mathrm{E}+01$ & $8.05 \mathrm{E}+00$ & core \\
\hline 146.4 & $2.10 \mathrm{E}+01$ & $5.82 \mathrm{E}+00$ & core \\
\hline 145.4 & $3.18 \mathrm{E}+01$ & $8.83 \mathrm{E}+00$ & core \\
\hline 144.4 & $1.42 \mathrm{E}+01$ & $3.95 \mathrm{E}+00$ & core \\
\hline 143.4 & $1.32 \mathrm{E}+01$ & $3.66 \mathrm{E}+00$ & core \\
\hline 142.4 & $2.52 \mathrm{E}+01$ & $7.01 \mathrm{E}+00$ & core \\
\hline 141.4 & $3.49 \mathrm{E}+01$ & $9.69 \mathrm{E}+00$ & core \\
\hline 140.4 & $3.93 \mathrm{E}+01$ & $1.09 \mathrm{E}+01$ & core \\
\hline 139.4 & $3.83 \mathrm{E}+01$ & $1.06 \mathrm{E}+01$ & core \\
\hline 138.4 & $3.93 \mathrm{E}+01$ & $1.09 \mathrm{E}+01$ & core \\
\hline 137.4 & $3.18 \mathrm{E}+01$ & $8.83 \mathrm{E}+00$ & core \\
\hline 136.4 & $5.44 \mathrm{E}+01$ & $1.51 \mathrm{E}+01$ & core \\
\hline 132.4 & $2.20 \mathrm{E}+01$ & $6.10 \mathrm{E}+00$ & core \\
\hline 131.4 & $2.30 \mathrm{E}+01$ & $6.39 \mathrm{E}+00$ & core \\
\hline 130.4 & $1.82 \mathrm{E}+01$ & $5.07 \mathrm{E}+00$ & core \\
\hline 127.4 & $5.44 \mathrm{E}+01$ & $1.51 \mathrm{E}+01$ & core \\
\hline 126.4 & $6.08 \mathrm{E}+01$ & $1.69 \mathrm{E}+01$ & core \\
\hline 125.4 & $6.67 \mathrm{E}+01$ & $1.85 \mathrm{E}+01$ & core \\
\hline 122.4 & $6.67 \mathrm{E}+01$ & $1.85 \mathrm{E}+01$ & core \\
\hline 120.4 & $4.60 \mathrm{E}+01$ & $1.28 \mathrm{E}+01$ & core \\
\hline $5.54 \mathrm{E}+01$ & $1.54 \mathrm{E}+01$ & core \\
\hline $4.60 \mathrm{E}+01$ & $1.28 \mathrm{E}+01$ & core \\
\hline
\end{tabular}


Conductivity Data at N13

\begin{tabular}{|c|c|c|c|}
\hline $\begin{array}{c}\text { Elevation } \\
\text { (ft) }\end{array}$ & $\begin{array}{c}\text { Kh } \\
\text { (ft/day) }\end{array}$ & $\begin{array}{c}\text { Kv } \\
\text { (ft/day) }\end{array}$ & $\begin{array}{c}\text { Data } \\
\text { Source }\end{array}$ \\
\hline 119.4 & $4.60 \mathrm{E}+01$ & $1.28 \mathrm{E}+01$ & core \\
\hline 118.4 & $6.67 \mathrm{E}+01$ & $1.85 \mathrm{E}+01$ & core \\
\hline 117.4 & $3.83 \mathrm{E}+01$ & $1.06 \mathrm{E}+01$ & core \\
\hline 116.4 & $3.83 \mathrm{E}+01$ & $1.06 \mathrm{E}+01$ & core \\
\hline 115.4 & $2.90 \mathrm{E}+01$ & $8.05 \mathrm{E}+00$ & core \\
\hline 114.4 & $1.15 \mathrm{E}+01$ & $3.19 \mathrm{E}+00$ & core \\
\hline 113.4 & $1.82 \mathrm{E}+01$ & $5.07 \mathrm{E}+00$ & core \\
\hline 112.4 & $1.15 \mathrm{E}+01$ & $3.19 \mathrm{E}+00$ & core \\
\hline 111.4 & $7.23 \mathrm{E}+00$ & $2.01 \mathrm{E}+00$ & core \\
\hline 110.4 & $7.23 \mathrm{E}+00$ & $2.01 \mathrm{E}+00$ & core \\
\hline 109.4 & $2.86 \mathrm{E}+00$ & $7.96 \mathrm{E}-01$ & core \\
\hline 108.4 & $1.82 \mathrm{E}+01$ & $5.07 \mathrm{E}+00$ & core \\
\hline 107.4 & $7.23 \mathrm{E}+00$ & $2.01 \mathrm{E}+00$ & core \\
\hline 106.4 & $2.72 \mathrm{E}-05$ & $7.56 \mathrm{E}-06$ & core \\
\hline 105.4 & $1.15 \mathrm{E}-04$ & $3.20 \mathrm{E}-05$ & core \\
\hline 104.4 & $2.90 \mathrm{E}+01$ & $8.05 \mathrm{E}+00$ & core \\
\hline 103.4 & $4.60 \mathrm{E}+01$ & $1.28 \mathrm{E}+01$ & core \\
\hline 102.4 & $5.54 \mathrm{E}+01$ & $1.54 \mathrm{E}+01$ & core \\
\hline 101.4 & $4.60 \mathrm{E}+01$ & $1.28 \mathrm{E}+01$ & core \\
\hline 100.4 & $4.60 \mathrm{E}+01$ & $1.28 \mathrm{E}+01$ & core \\
\hline 99.4 & $4.60 \mathrm{E}+01$ & $1.28 \mathrm{E}+01$ & core \\
\hline 98.4 & $4.60 \mathrm{E}+01$ & $1.28 \mathrm{E}+01$ & core \\
\hline 97.4 & $4.60 \mathrm{E}+01$ & $1.28 \mathrm{E}+01$ & core \\
\hline 96.4 & $5.54 \mathrm{E}+01$ & $1.54 \mathrm{E}+01$ & core \\
\hline 95.4 & $4.60 \mathrm{E}+01$ & $1.28 \mathrm{E}+01$ & core \\
\hline 94.4 & $6.08 \mathrm{E}+01$ & $1.69 \mathrm{E}+01$ & core \\
\hline 92.4 & $6.08 \mathrm{E}+01$ & $1.69 \mathrm{E}+01$ & core \\
\hline 91.4 & $6.08 \mathrm{E}+01$ & $1.69 \mathrm{E}+01$ & core \\
\hline 90.4 & $4.60 \mathrm{E}+01$ & $1.28 \mathrm{E}+01$ & core \\
\hline 89.4 & $2.90 \mathrm{E}+01$ & $8.05 \mathrm{E}+00$ & core \\
\hline 88.4 & $3.83 \mathrm{E}+01$ & $1.06 \mathrm{E}+01$ & core \\
\hline 87.4 & $3.83 \mathrm{E}+01$ & $1.06 \mathrm{E}+01$ & core \\
\hline 86.4 & $7.23 \mathrm{E}+00$ & $2.01 \mathrm{E}+00$ & core \\
\hline 85.4 & $2.86 \mathrm{E}+00$ & $7.96 \mathrm{E}-01$ & core \\
\hline 84.4 & $4.60 \mathrm{E}+01$ & $1.28 \mathrm{E}+01$ & core \\
\hline 83.4 & $5.54 \mathrm{E}+01$ & $1.54 \mathrm{E}+01$ & core \\
\hline 82.4 & $6.08 \mathrm{E}+01$ & $1.69 \mathrm{E}+01$ & core \\
\hline 81.4 & $6.08 \mathrm{E}+01$ & $1.69 \mathrm{E}+01$ & core \\
\hline 77.4 & $5.54 \mathrm{E}+01$ & $1.54 \mathrm{E}+01$ & core \\
\hline 76.4 & $6.08 \mathrm{E}+01$ & $1.69 \mathrm{E}+01$ & core \\
\hline 67.4 & $5.54 \mathrm{E}+01$ & $1.54 \mathrm{E}+01$ & core \\
\hline 66.4 & $5.54 \mathrm{E}+01$ & $1.54 \mathrm{E}+01$ & core \\
\hline 65.4 & $5.54 \mathrm{E}+01$ & $1.54 \mathrm{E}+01$ & core \\
\hline 62.4 & $6.67 \mathrm{E}+01$ & $1.85 \mathrm{E}+01$ & core \\
\hline 60.4 & $6.67 \mathrm{E}+01$ & $1.85 \mathrm{E}+01$ & core \\
\hline 59.4 & $2.57 \mathrm{E}-05$ & $7.14 \mathrm{E}-06$ & core \\
\hline 58.4 & $2.43 \mathrm{E}-05$ & $6.74 \mathrm{E}-06$ & core \\
\hline & & $6.74 \mathrm{E}-06$ & core \\
\hline
\end{tabular}


Conductivity Data at N13

\begin{tabular}{|c|c|c|c|}
\hline $\begin{array}{c}\text { Elevation } \\
\text { (ft) }\end{array}$ & $\begin{array}{c}\text { Kh } \\
\text { (ft/day) }\end{array}$ & $\begin{array}{c}\text { Kv } \\
\text { (ft/day) }\end{array}$ & $\begin{array}{l}\text { Data } \\
\text { Source }\end{array}$ \\
\hline 57.4 & $2.86 \mathrm{E}+00$ & $7.96 \mathrm{E}-01$ & core \\
\hline 56.4 & $2.90 E+01$ & $8.05 E+00$ & core \\
\hline 55.4 & $3.83 E+01$ & $1.06 E+01$ & core \\
\hline 54.4 & $3.83 \mathrm{E}+01$ & $1.06 \mathrm{E}+01$ & core \\
\hline 52.4 & $2.90 \mathrm{E}+01$ & $8.05 E+00$ & core \\
\hline 51.4 & $5.54 \mathrm{E}+01$ & $1.54 E+01$ & core \\
\hline 50.4 & $5.54 \mathrm{E}+01$ & $1.54 \mathrm{E}+01$ & core \\
\hline 49.4 & $5.54 E+01$ & $1.54 \mathrm{E}+01$ & core \\
\hline 48.4 & $5.54 \mathrm{E}+01$ & $1.54 \mathrm{E}+01$ & core \\
\hline 43.4 & $4.60 \mathrm{E}+01$ & $1.28 E+01$ & core \\
\hline 40.4 & $4.60 \mathrm{E}+01$ & $1.28 \mathrm{E}+01$ & core \\
\hline 39.4 & $4.60 \mathrm{E}+01$ & $1.28 \mathrm{E}+01$ & core \\
\hline 37.4 & $6.08 \mathrm{E}+01$ & $1.69 \mathrm{E}+01$ & core \\
\hline 36.4 & $6.08 \mathrm{E}+01$ & $1.69 E+01$ & core \\
\hline 35.4 & $6.67 \mathrm{E}+01$ & $1.85 \mathrm{E}+01$ & core \\
\hline 34.4 & $4.60 \mathrm{E}+01$ & $1.28 \mathrm{E}+01$ & core \\
\hline 33.4 & $5.54 \mathrm{E}+01$ & $1.54 \mathrm{E}+01$ & core \\
\hline 32.4 & $5.54 \mathrm{E}+01$ & $1.54 \mathrm{E}+01$ & core \\
\hline 31.4 & $5.54 \mathrm{E}+01$ & $1.54 \mathrm{E}+01$ & core \\
\hline 30.4 & $5.54 \mathrm{E}+01$ & $1.54 \mathrm{E}+01$ & core \\
\hline 29.4 & $4.41 E-05$ & $1.23 \mathrm{E}-05$ & core \\
\hline 27.4 & $3.20 \mathrm{E}-05$ & $8.90 \mathrm{E}-06$ & core \\
\hline 23.4 & 8.36E-05 & $2.32 \mathrm{E}-05$ & core \\
\hline 17.4 & $6.08 \mathrm{E}+01$ & $1.69 E+01$ & core \\
\hline 16.4 & $6.67 E+01$ & $1.85 E+01$ & core \\
\hline 15.4 & $6.67 E+01$ & $1.85 E+01$ & core \\
\hline 14.4 & $6.67 E+01$ & $1.85 E+01$ & core \\
\hline 12.4 & $6.67 E+01$ & $1.85 \mathrm{E}+01$ & core \\
\hline 11.4 & $6.67 \mathrm{E}+01$ & $1.85 E+01$ & core \\
\hline 10.4 & $6.67 E+01$ & $1.85 E+01$ & core \\
\hline 9.4 & $6.67 \mathrm{E}+01$ & $1.85 \mathrm{E}+01$ & core \\
\hline 8.4 & $6.67 \mathrm{E}+01$ & $1.85 E+01$ & core \\
\hline 7.4 & 4.41E-05 & $1.23 \mathrm{E}-05$ & core \\
\hline-2.6 & $2.57 \mathrm{E}-05$ & $7.14 \mathrm{E}-06$ & core \\
\hline-3.6 & $4.55 \mathrm{E}+00$ & $1.26 \mathrm{E}+00$ & core \\
\hline-4.6 & $2.90 \mathrm{E}+01$ & $8.05 E+00$ & core \\
\hline-5.6 & $2.57 \mathrm{E}-05$ & $7.14 \mathrm{E}-06$ & core \\
\hline-6.6 & $2.72 \mathrm{E}-05$ & $7.56 \mathrm{E}-06$ & core \\
\hline
\end{tabular}


Conductivity Data at P20

\begin{tabular}{|c|c|c|c|}
\hline $\begin{array}{c}\text { Elevation } \\
\text { (ft) }\end{array}$ & $\begin{array}{c}\text { Kh } \\
\text { (ft/day) }\end{array}$ & $\begin{array}{c}\mathrm{Kv} \\
\text { (ft/day) }\end{array}$ & $\begin{array}{l}\text { Data } \\
\text { Source }\end{array}$ \\
\hline 278.2 & $1.79 \mathrm{E}-01$ & $4.97 \mathrm{E}-02$ & core \\
\hline 277.2 & $2.84 \mathrm{E}-01$ & $7.89 \mathrm{E}-02$ & core \\
\hline 276.2 & $1.13 \mathrm{E}-01$ & $3.13 \mathrm{E}-02$ & core \\
\hline 275.2 & $7.09 E-02$ & $1.97 E-02$ & core \\
\hline 274.2 & $1.13 \mathrm{E}-01$ & $3.13 E-02$ & core \\
\hline 273.2 & 2.81E-02 & $7.81 E-03$ & core \\
\hline 272.2 & $4.46 \mathrm{E}-02$ & $1.24 \mathrm{E}-02$ & core \\
\hline 271.2 & $1.13 \mathrm{E}-01$ & $3.13 E-02$ & core \\
\hline 270.2 & 1.79E-01 & 4.97E-02 & core \\
\hline 269.2 & $2.81 E-02$ & 7.81E-03 & core \\
\hline 268.2 & $4.51 \mathrm{E}-01$ & $1.25 E-01$ & core \\
\hline 267.2 & $2.81 \mathrm{E}-02$ & $7.81 E-03$ & core \\
\hline 266.2 & $4.46 \mathrm{E}-02$ & $1.24 E-02$ & core \\
\hline 265.2 & 2.84E-01 & $7.89 E-02$ & core \\
\hline 264.2 & $2.84 E-01$ & $7.89 \mathrm{E}-02$ & core \\
\hline 260.2 & $1.13 \mathrm{E}-01$ & 3.13E-02 & core \\
\hline 259.2 & $1.13 \mathrm{E}-01$ & $3.13 E-02$ & core \\
\hline 256.2 & $1.79 E-01$ & 4.97E-02 & core \\
\hline 253.2 & $2.84 \mathrm{E}-01$ & 7.89E-02 & core \\
\hline 252.2 & $1.14 \mathrm{E}+00$ & $3.16 \mathrm{E}-01$ & core \\
\hline 250.2 & $4.51 \mathrm{E}-01$ & $1.25 \mathrm{E}-01$ & core \\
\hline 249.2 & $1.14 \mathrm{E}+00$ & $3.16 \mathrm{E}-01$ & core \\
\hline 248.2 & $1.81 E+00$ & $5.02 \mathrm{E}-01$ & core \\
\hline 247.2 & $1.81 E+00$ & $5.02 \mathrm{E}-01$ & core \\
\hline 246.2 & $4.56 E+00$ & $1.27 E+00$ & core \\
\hline 244.2 & $4.56 E+00$ & $1.27 E+00$ & core \\
\hline 243.2 & $2.87 E+00$ & 7.97E-01 & core \\
\hline 242.2 & $4.56 E+00$ & $1.27 E+00$ & core \\
\hline 241.2 & $4.56 E+00$ & $1.27 E+00$ & core \\
\hline 240.2 & $2.87 E+00$ & 7.97E-01 & core \\
\hline 239.2 & $1.81 E+00$ & $5.02 E-01$ & core \\
\hline 238.2 & $1.81 \mathrm{E}+00$ & $5.02 E-01$ & core \\
\hline 237.2 & $2.84 \mathrm{E}-01$ & $7.89 E-02$ & core \\
\hline 236.2 & $7.24 E+00$ & $2.01 E+00$ & core \\
\hline 235.2 & $4.56 E+00$ & $1.27 E+00$ & core \\
\hline 234.2 & $1.81 E+00$ & $5.02 E-01$ & core \\
\hline 233.2 & $2.87 E+00$ & $7.97 \mathrm{E}-01$ & core \\
\hline 232.2 & $2.84 \mathrm{E}-01$ & $7.89 E-02$ & core \\
\hline 231.2 & $2.87 E+00$ & $7.97 \mathrm{E}-01$ & core \\
\hline 230.2 & $2.87 E+00$ & $7.97 \mathrm{E}-01$ & core \\
\hline 229.2 & $1.81 E+00$ & $5.02 E-01$ & core \\
\hline 228.2 & $2.87 E+00$ & 7.97E-01 & core \\
\hline 226.2 & $2.87 E+00$ & 7.97E-01 & core \\
\hline 225.2 & $1.81 E+00$ & $5.02 E-01$ & core \\
\hline 224.2 & $2.87 E+00$ & 7.97E-01 & core \\
\hline 223.2 & $2.87 E+00$ & $7.97 E-01$ & core \\
\hline 222.2 & $1.81 E+00$ & $5.02 E-01$ & core \\
\hline 221.2 & $1.81 E+00$ & $5.02 E-01$ & core \\
\hline
\end{tabular}


Conductivity Data at P20

\begin{tabular}{|c|c|c|c|}
\hline $\begin{array}{c}\text { Elevation } \\
\text { (ft) }\end{array}$ & $\begin{array}{c}\text { Kh } \\
\text { (ft/day) }\end{array}$ & $\begin{array}{c}\text { Kv } \\
\text { (ft/day) }\end{array}$ & $\begin{array}{c}\text { Data } \\
\text { Source }\end{array}$ \\
\hline 220.2 & $2.84 \mathrm{E}-01$ & $7.89 \mathrm{E}-02$ & core \\
\hline 219.2 & $1.81 \mathrm{E}+00$ & $5.02 \mathrm{E}-01$ & core \\
\hline 218.2 & $1.81 \mathrm{E}+00$ & $5.02 \mathrm{E}-01$ & core \\
\hline 217.2 & $4.51 \mathrm{E}-01$ & $1.25 \mathrm{E}-01$ & core \\
\hline 216.2 & $2.87 \mathrm{E}+00$ & $7.97 \mathrm{E}-01$ & core \\
\hline 215.2 & $5.28 \mathrm{E}-04$ & $1.47 \mathrm{E}-04$ & core \\
\hline 214.2 & $8.91 \mathrm{E}-04$ & $2.48 \mathrm{E}-04$ & core \\
\hline 213.2 & $1.50 \mathrm{E}-03$ & $4.18 \mathrm{E}-04$ & core \\
\hline 212.2 & $1.50 \mathrm{E}-03$ & $4.18 \mathrm{E}-04$ & core \\
\hline 211.2 & $1.39 \mathrm{E}-01$ & $3.86 \mathrm{E}-02$ & core \\
\hline 210.2 & $2.54 \mathrm{E}-03$ & $7.05 \mathrm{E}-04$ & core \\
\hline 209.2 & $1.50 \mathrm{E}-03$ & $4.18 \mathrm{E}-04$ & core \\
\hline 208.2 & $8.91 \mathrm{E}-04$ & $2.48 \mathrm{E}-04$ & core \\
\hline 207.2 & $1.50 \mathrm{E}-03$ & $4.18 \mathrm{E}-04$ & core \\
\hline 206.2 & $2.54 \mathrm{E}-03$ & $7.05 \mathrm{E}-04$ & core \\
\hline 205.2 & $5.28 \mathrm{E}-04$ & $1.47 \mathrm{E}-04$ & core \\
\hline 204.2 & $6.07 \mathrm{E}-05$ & $1.69 \mathrm{E}-05$ & core \\
\hline 203.2 & $5.28 \mathrm{E}-04$ & $1.47 \mathrm{E}-04$ & core \\
\hline 202.2 & $2.89 \mathrm{E}+01$ & $8.04 \mathrm{E}+00$ & core \\
\hline 201.2 & $2.89 \mathrm{E}+01$ & $8.04 \mathrm{E}+00$ & core \\
\hline 200.2 & $4.60 \mathrm{E}+01$ & $1.28 \mathrm{E}+01$ & core \\
\hline 199.2 & $4.60 \mathrm{E}+01$ & $1.28 \mathrm{E}+01$ & core \\
\hline 198.2 & $2.89 \mathrm{E}+01$ & $8.04 \mathrm{E}+00$ & core \\
\hline 197.2 & $1.82 \mathrm{E}+01$ & $5.06 \mathrm{E}+00$ & core \\
\hline 196.2 & $2.89 \mathrm{E}+01$ & $8.04 \mathrm{E}+00$ & core \\
\hline 195.2 & $5.28 \mathrm{E}-04$ & $1.47 \mathrm{E}-04$ & core \\
\hline 194.2 & $5.28 \mathrm{E}-04$ & $1.47 \mathrm{E}-04$ & core \\
\hline 193.2 & $1.59 \mathrm{E}-04$ & $4.40 \mathrm{E}-05$ & core \\
\hline 191.2 & $1.39 \mathrm{E}-01$ & $3.86 \mathrm{E}-02$ & core \\
\hline 186.2 & $5.28 \mathrm{E}-04$ & $1.47 \mathrm{E}-04$ & core \\
\hline 185.2 & $5.28 \mathrm{E}-04$ & $1.47 \mathrm{E}-04$ & core \\
\hline 184.2 & $5.28 \mathrm{E}-04$ & $1.47 \mathrm{E}-04$ & core \\
\hline 183.2 & $1.15 \mathrm{E}-04$ & $3.20 \mathrm{E}-05$ & core \\
\hline 181.7 & $3.12 \mathrm{E}-04$ & $9.64 \mathrm{E}-05$ & perm \\
\hline 180.2 & $3.13 \mathrm{E}-04$ & $8.69 \mathrm{E}-05$ & core \\
\hline 179.2 & $1.15 \mathrm{E}-04$ & $3.20 \mathrm{E}-05$ & core \\
\hline 178.2 & $1.15 \mathrm{E}-04$ & $3.20 \mathrm{E}-05$ & core \\
\hline 177.2 & $3.20 \mathrm{E}-05$ & $8.90 \mathrm{E}-06$ & core \\
\hline 176.2 & $5.28 \mathrm{E}-04$ & $1.47 \mathrm{E}-04$ & core \\
\hline 175.2 & $1.15 \mathrm{E}-04$ & $3.20 \mathrm{E}-05$ & core \\
\hline 174.2 & $1.59 \mathrm{E}-04$ & $4.40 \mathrm{E}-05$ & core \\
\hline 173.2 & $5.28 \mathrm{E}-04$ & $1.47 \mathrm{E}-04$ & core \\
\hline 172.2 & $5.28 \mathrm{E}-04$ & $1.47 \mathrm{E}-04$ & core \\
\hline 171.2 & $8.91 \mathrm{E}-04$ & $2.48 \mathrm{E}-04$ & core \\
\hline 170.2 & $8.91 \mathrm{E}-04$ & $2.48 \mathrm{E}-04$ & core \\
\hline 169.2 & $5.28 \mathrm{E}-04$ & $1.47 \mathrm{E}-04$ & core \\
\hline 167.2 & $1.39 \mathrm{E}-01$ & $3.86 \mathrm{E}-02$ & core \\
\hline $2.18 \mathrm{E}-04$ & $6.06 \mathrm{E}-05$ & core \\
\hline
\end{tabular}


Conductivity Data at P20

\begin{tabular}{|c|c|c|c|}
\hline $\begin{array}{c}\text { Elevation } \\
\text { (ft) }\end{array}$ & $\begin{array}{c}\text { Kh } \\
\text { (ft/day) }\end{array}$ & $\begin{array}{c}\mathrm{Kv} \\
\text { (ft/day) }\end{array}$ & $\begin{array}{c}\text { Data } \\
\text { Source }\end{array}$ \\
\hline 166.2 & $5.28 \mathrm{E}-04$ & $1.47 E-04$ & core \\
\hline 165.2 & $5.28 \mathrm{E}-04$ & 1.47E-04 & core \\
\hline 164.2 & $1.50 \mathrm{E}-03$ & $4.18 E-04$ & core \\
\hline 163.2 & $2.54 \mathrm{E}-03$ & $7.05 E-04$ & core \\
\hline 162.2 & 8.91E-04 & $2.48 E-04$ & core \\
\hline 161.2 & $1.50 \mathrm{E}-03$ & $4.18 E-04$ & core \\
\hline 160.2 & 8.91E-04 & $2.48 E-04$ & core \\
\hline 159.2 & 8.91E-04 & $2.48 E-04$ & core \\
\hline 158.2 & $5.28 \mathrm{E}-04$ & $1.47 E-04$ & core \\
\hline 157.2 & $8.36 \mathrm{E}-05$ & $2.32 E-05$ & core \\
\hline 156.2 & $2.72 E-05$ & $7.56 \mathrm{E}-06$ & core \\
\hline 155.2 & 4.41E-05 & $1.23 E-05$ & core \\
\hline 154.2 & $5.28 \mathrm{E}-04$ & $1.47 E-04$ & core \\
\hline 153.2 & 8.91E-04 & $2.48 E-04$ & core \\
\hline 152.2 & $8.91 E-04$ & $2.48 E-04$ & core \\
\hline 151.2 & $1.15 E+01$ & $3.19 E+00$ & core \\
\hline 150.2 & $1.82 E+01$ & $5.07 E+00$ & core \\
\hline 149.2 & $2.90 \mathrm{E}+01$ & $8.05 E+00$ & core \\
\hline 148.2 & $7.23 E+00$ & $2.01 E+00$ & core \\
\hline 146.2 & $4.55 E+00$ & $1.26 \mathrm{E}+00$ & core \\
\hline 145.2 & $2.86 E+00$ & $7.96 \mathrm{E}-01$ & core \\
\hline 144.2 & $1.15 \mathrm{E}+01$ & $3.19 E+00$ & core \\
\hline 143.2 & $1.15 E+01$ & $3.19 \mathrm{E}+00$ & core \\
\hline 141.2 & $1.82 \mathrm{E}+01$ & $5.07 \mathrm{E}+00$ & core \\
\hline 140.2 & $4.55 E+00$ & $1.26 \mathrm{E}+00$ & core \\
\hline 139.2 & $1.80 \mathrm{E}+00$ & 5.01E-01 & core \\
\hline 136.2 & $7.23 E+00$ & $2.01 \mathrm{E}+00$ & core \\
\hline 131.2 & 1.21E-03 & 3.37E-04 & core \\
\hline 130.2 & $2.18 \mathrm{E}-04$ & $6.06 \mathrm{E}-05$ & core \\
\hline 129.2 & $1.15 \mathrm{E}+01$ & $3.19 E+00$ & core \\
\hline 126.2 & $1.15 \mathrm{E}+01$ & $3.19 E+00$ & core \\
\hline 125.2 & $1.80 E+00$ & 5.01E-01 & core \\
\hline 124.2 & $2.86 \mathrm{E}+00$ & $7.96 \mathrm{E}-01$ & core \\
\hline 123.2 & $2.18 E-04$ & $6.06 \mathrm{E}-05$ & core \\
\hline 122.2 & $1.15 \mathrm{E}-04$ & $3.20 E-05$ & core \\
\hline 121.2 & $4.55 \mathrm{E}+00$ & $1.26 \mathrm{E}+00$ & core \\
\hline 120.2 & $2.90 E+01$ & $8.05 \mathrm{E}+00$ & core \\
\hline 119.2 & $2.07 E+00$ & $5.75 E-01$ & core \\
\hline 118.2 & $2.05 E-04$ & $5.69 E-05$ & core \\
\hline 116.2 & $2.18 E-04$ & $6.06 E-05$ & core \\
\hline 115.2 & $1.80 \mathrm{E}+00$ & $5.01 \mathrm{E}-01$ & core \\
\hline 114.2 & $3.45 E+00$ & 9.57E-01 & core \\
\hline 113.2 & $2.27 E+00$ & $6.31 E-01$ & core \\
\hline 112.2 & $4.77 E+00$ & $1.32 E+00$ & core \\
\hline 111.2 & 1.86E-04 & 5.17E-05 & core \\
\hline 110.2 & $3.38 E-03$ & $9.38 E-04$ & core \\
\hline 109.2 & $2.73 E-04$ & $7.58 \mathrm{E}-05$ & core \\
\hline 108.2 & $1.54 \mathrm{E}-04$ & $4.26 \mathrm{E}-05$ & core \\
\hline
\end{tabular}


Conductivity Data at P20

\begin{tabular}{|c|c|c|c|}
\hline $\begin{array}{c}\text { Elevation } \\
\text { (ft) }\end{array}$ & $\begin{array}{c}\text { Kh } \\
\text { (ft/day) }\end{array}$ & $\begin{array}{c}\text { Kv } \\
\text { (ft/day) }\end{array}$ & $\begin{array}{c}\text { Data } \\
\text { Source }\end{array}$ \\
\hline 107.2 & $2.02 \mathrm{E}-01$ & $5.60 \mathrm{E}-02$ & core \\
\hline 106.2 & $1.92 \mathrm{E}-04$ & $5.33 \mathrm{E}-05$ & core \\
\hline 105.2 & $1.35 \mathrm{E}-04$ & $3.75 \mathrm{E}-05$ & core \\
\hline 104.2 & $2.18 \mathrm{E}-04$ & $6.06 \mathrm{E}-05$ & core \\
\hline 101.2 & $6.49 \mathrm{E}-01$ & $1.80 \mathrm{E}-01$ & core \\
\hline 100.2 & $1.72 \mathrm{E}-04$ & $4.78 \mathrm{E}-05$ & core \\
\hline 99.2 & $2.17 \mathrm{E}+00$ & $6.03 \mathrm{E}-01$ & core \\
\hline 98.2 & $5.23 \mathrm{E}-03$ & $1.45 \mathrm{E}-03$ & core \\
\hline 95.2 & $8.20 \mathrm{E}-05$ & $2.28 \mathrm{E}-05$ & core \\
\hline 91.2 & $9.71 \mathrm{E}-02$ & $2.70 \mathrm{E}-02$ & core \\
\hline 90.2 & $1.92 \mathrm{E}-04$ & $5.33 \mathrm{E}-05$ & core \\
\hline 89.2 & $2.49 \mathrm{E}+00$ & $6.93 \mathrm{E}-01$ & core \\
\hline 88.2 & $3.78 \mathrm{E}+00$ & $1.05 \mathrm{E}+00$ & core \\
\hline 87.2 & $6.59 \mathrm{E}+00$ & $1.83 \mathrm{E}+00$ & core \\
\hline 86.2 & $4.55 \mathrm{E}+00$ & $1.26 \mathrm{E}+00$ & core \\
\hline 85.2 & $3.78 \mathrm{E}+00$ & $1.05 \mathrm{E}+00$ & core \\
\hline 84.2 & $2.17 \mathrm{E}+00$ & $6.03 \mathrm{E}-01$ & core \\
\hline 83.2 & $1.92 \mathrm{E}-04$ & $5.33 \mathrm{E}-05$ & core \\
\hline 82.2 & $5.23 \mathrm{E}-03$ & $1.45 \mathrm{E}-03$ & core \\
\hline 81.2 & $1.31 \mathrm{E}-04$ & $3.63 \mathrm{E}-05$ & core \\
\hline 80.2 & $2.57 \mathrm{E}-05$ & $7.14 \mathrm{E}-06$ & core \\
\hline 79.2 & $2.57 \mathrm{E}-05$ & $7.14 \mathrm{E}-06$ & core \\
\hline 78.2 & $2.32 \mathrm{E}-05$ & $6.43 \mathrm{E}-06$ & core \\
\hline 77.2 & $3.12 \mathrm{E}-03$ & $2.58 \mathrm{E}-03$ & perm \\
\hline 76.2 & $2.43 \mathrm{E}-05$ & $6.74 \mathrm{E}-06$ & core \\
\hline 75.2 & $1.15 \mathrm{E}-04$ & $3.20 \mathrm{E}-05$ & core \\
\hline 74.2 & $1.15 \mathrm{E}-04$ & $3.20 \mathrm{E}-05$ & core \\
\hline 73.2 & $1.80 \mathrm{E}+00$ & $5.01 \mathrm{E}-01$ & core \\
\hline 72.2 & $1.15 \mathrm{E}-04$ & $3.20 \mathrm{E}-05$ & core \\
\hline 71.2 & $2.86 \mathrm{E}+00$ & $7.96 \mathrm{E}-01$ & core \\
\hline 70.2 & $1.15 \mathrm{E}-04$ & $3.20 \mathrm{E}-05$ & core \\
\hline 69.2 & $1.80 \mathrm{E}+00$ & $5.01 \mathrm{E}-01$ & core \\
\hline 68.2 & $2.86 \mathrm{E}+00$ & $7.96 \mathrm{E}-01$ & core \\
\hline 67.2 & $2.86 \mathrm{E}+00$ & $7.96 \mathrm{E}-01$ & core \\
\hline 66.2 & $4.55 \mathrm{E}+00$ & $1.26 \mathrm{E}+00$ & core \\
\hline 65.2 & $1.15 \mathrm{E}+01$ & $3.19 \mathrm{E}+00$ & core \\
\hline 64.2 & $1.15 \mathrm{E}+01$ & $3.19 \mathrm{E}+00$ & core \\
\hline 63.2 & $2.86 \mathrm{E}+00$ & $7.96 \mathrm{E}-01$ & core \\
\hline 62.2 & $4.55 \mathrm{E}+00$ & $1.26 \mathrm{E}+00$ & core \\
\hline 61.2 & $4.55 \mathrm{E}+00$ & $1.26 \mathrm{E}+00$ & core \\
\hline 60.2 & $1.82 \mathrm{E}+01$ & $5.07 \mathrm{E}+00$ & core \\
\hline 59.2 & $7.23 \mathrm{E}+00$ & $2.01 \mathrm{E}+00$ & core \\
\hline 58.2 & $1.80 \mathrm{E}+00$ & $5.01 \mathrm{E}-01$ & core \\
\hline 57.2 & $1.82 \mathrm{E}+01$ & $5.07 \mathrm{E}+00$ & core \\
\hline 56.2 & $1.82 \mathrm{E}+01$ & $5.07 \mathrm{E}+00$ & core \\
\hline 55.2 & $1.82 \mathrm{E}+01$ & $5.07 \mathrm{E}+00$ & core \\
\hline 54.2 & $7.23 \mathrm{E}+00$ & $2.01 \mathrm{E}+00$ & core \\
\hline 53.2 & $1.15 \mathrm{E}+01$ & $3.19 \mathrm{E}+00$ & core \\
\hline & & & \\
\hline 50 & & \\
\hline
\end{tabular}


Conductivity Data at P20

\begin{tabular}{|c|c|c|c|}
\hline $\begin{array}{c}\text { Elevation } \\
\text { (ft) }\end{array}$ & $\begin{array}{c}\text { Kh } \\
\text { (ft/day) }\end{array}$ & $\begin{array}{c}\text { Kv } \\
\text { (ft/day) }\end{array}$ & $\begin{array}{l}\text { Data } \\
\text { Source }\end{array}$ \\
\hline 52.2 & $2.90 \mathrm{E}+01$ & $8.05 E+00$ & core \\
\hline 51.2 & $2.90 E+01$ & $8.05 E+00$ & core \\
\hline 50.2 & $1.82 E+01$ & $5.07 E+00$ & core \\
\hline 48.2 & $2.90 E+01$ & $8.05 E+00$ & core \\
\hline 47.2 & $2.90 E+01$ & $8.05 E+00$ & core \\
\hline 46.2 & $2.90 E+01$ & $8.05 E+00$ & core \\
\hline 45.2 & $2.90 E+01$ & $8.05 E+00$ & core \\
\hline 44.2 & $2.90 E+01$ & $8.05 E+00$ & core \\
\hline 43.2 & $1.15 E+01$ & $3.19 E+00$ & core \\
\hline 42.2 & $7.23 E+00$ & $2.01 \mathrm{E}+00$ & core \\
\hline 41.2 & $2.90 \mathrm{E}+01$ & $8.05 E+00$ & core \\
\hline 40.2 & $2.90 E+01$ & $8.05 E+00$ & core \\
\hline 39.2 & $4.60 E+01$ & $1.28 \mathrm{E}+01$ & core \\
\hline 38.2 & $1.82 E+01$ & $5.07 E+00$ & core \\
\hline 37.2 & $4.55 E+00$ & $1.26 E+00$ & core \\
\hline 36.2 & $1.15 \mathrm{E}+01$ & $3.19 E+00$ & core \\
\hline 34.2 & $2.90 E+01$ & $8.05 E+00$ & core \\
\hline 33.2 & $4.60 \mathrm{E}+01$ & $1.28 \mathrm{E}+01$ & core \\
\hline 31.2 & $4.60 \mathrm{E}+01$ & $1.28 \mathrm{E}+01$ & core \\
\hline 30.2 & $4.60 \mathrm{E}+01$ & $1.28 \mathrm{E}+01$ & core \\
\hline 29.2 & $1.82 \mathrm{E}+01$ & $5.07 E+00$ & core \\
\hline 28.2 & $1.82 \mathrm{E}+01$ & $5.07 E+00$ & core \\
\hline 27.2 & $1.82 \mathrm{E}+01$ & $5.07 E+00$ & core \\
\hline 26.2 & $2.90 E+01$ & $8.05 E+00$ & core \\
\hline 25.2 & $1.82 E+01$ & $5.07 E+00$ & core \\
\hline 24.2 & $1.82 \mathrm{E}+01$ & $5.07 E+00$ & core \\
\hline 23.2 & $1.82 \mathrm{E}+01$ & $5.07 E+00$ & core \\
\hline 22.2 & $1.82 E+01$ & $5.07 E+00$ & core \\
\hline 21.2 & $1.82 E+01$ & $5.07 E+00$ & core \\
\hline 20.2 & $2.90 \mathrm{E}+01$ & $8.05 E+00$ & core \\
\hline 19.2 & $4.60 E+01$ & $1.28 E+01$ & core \\
\hline 18.2 & $1.82 E+01$ & $5.07 E+00$ & core \\
\hline 17.2 & $1.82 \mathrm{E}+01$ & $5.07 E+00$ & core \\
\hline 16.2 & $7.23 E+00$ & $2.01 E+00$ & core \\
\hline 15.2 & $2.90 E+01$ & $8.05 E+00$ & core \\
\hline 14.2 & $1.15 \mathrm{E}+01$ & $3.19 E+00$ & core \\
\hline 13.2 & $2.90 \mathrm{E}+01$ & $8.05 E+00$ & core \\
\hline 10.2 & $1.82 \mathrm{E}+01$ & $5.07 E+00$ & core \\
\hline 9.2 & $1.82 E+01$ & $5.07 E+00$ & core \\
\hline 8.2 & $2.90 \mathrm{E}+01$ & $8.05 E+00$ & core \\
\hline 7.2 & $1.15 E+01$ & $3.19 E+00$ & core \\
\hline 6.2 & $1.15 E+01$ & $3.19 E+00$ & core \\
\hline 5.2 & $1.82 \mathrm{E}+01$ & $5.07 E+00$ & core \\
\hline 4.2 & $2.90 E+01$ & $8.05 E+00$ & core \\
\hline 3.2 & $7.23 E+00$ & $2.01 E+00$ & core \\
\hline 2.2 & $2.90 E+01$ & $8: 05 E+00$ & core \\
\hline 1.2 & $4.60 \mathrm{E}+01$ & $1.28 \mathrm{E}+01$ & core \\
\hline-1.8 & $2.90 E+01$ & $8.05 E+00$ & core \\
\hline
\end{tabular}


Conductivity Data at P20

\begin{tabular}{|c|c|c|c|}
\hline $\begin{array}{c}\text { Elevation } \\
\text { (ft) }\end{array}$ & $\begin{array}{c}\mathrm{Kh} \\
\text { (ft/day) }\end{array}$ & $\begin{array}{c}\mathrm{Kv} \\
\text { (ft/day) }\end{array}$ & $\begin{array}{c}\text { Data } \\
\text { Source }\end{array}$ \\
\hline-2.8 & $1.82 \mathrm{E}+01$ & $5.07 \mathrm{E}+00$ & core \\
\hline-3.8 & $1.82 \mathrm{E}+01$ & $5.07 \mathrm{E}+00$ & core \\
\hline-4.8 & $4.60 E+01$ & $1.28 \mathrm{E}+01$ & core \\
\hline-5.8 & $4.60 E+01$ & $1.28 \mathrm{E}+01$ & core \\
\hline-6.8 & $2.90 \mathrm{E}+01$ & $8.05 E+00$ & core \\
\hline-8.8 & $2.90 E+01$ & $8.05 E+00$ & core \\
\hline-9.8 & $4.60 \mathrm{E}+01$ & $1.28 \mathrm{E}+01$ & core \\
\hline-10.8 & $2.43 E-05$ & $6.74 \mathrm{E}-06$ & core \\
\hline-11.8 & $2.32 \mathrm{E}-05$ & $6.43 E-06$ & core \\
\hline-12.8 & 2.32E-05 & $6.43 E-06$ & core \\
\hline-13.8 & $2.43 \mathrm{E}-05$ & 6.74E-06 & core \\
\hline-14.8 & $2.57 \mathrm{E}-05$ & $7.14 \mathrm{E}-06$ & core \\
\hline-15.8 & $2.72 E-05$ & $7.56 \mathrm{E}-06$ & core \\
\hline-16.8 & $2.43 E-05$ & $6.74 \mathrm{E}-06$ & core \\
\hline-17.8 & $2.32 E-05$ & $6.43 \mathrm{E}-06$ & core \\
\hline-18.8 & $2.32 E-05$ & $6.43 E-06$ & core \\
\hline-19.8 & $2.34 E-05$ & $6.51 \mathrm{E}-06$ & core \\
\hline-20.8 & $2.32 E-05$ & $6.43 \mathrm{E}-06$ & core \\
\hline-21.8 & $2.32 E-05$ & $6.43 E-06$ & core \\
\hline-22.8 & $2.34 \mathrm{E}-05$ & $6.51 \mathrm{E}-06$ & core \\
\hline-23.8 & $2.32 \mathrm{E}-05$ & $6.43 \mathrm{E}-06$ & core \\
\hline-24.8 & $2.32 E-05$ & $6.43 E-06$ & core \\
\hline-25.8 & $2.32 \mathrm{E}-05$ & $6.43 \mathrm{E}-06$ & core \\
\hline-26.8 & $2.32 \mathrm{E}-05$ & $6.43 \mathrm{E}-06$ & core \\
\hline-27.8 & $2.32 E-05$ & $6.43 \mathrm{E}-06$ & core \\
\hline-28.8 & $2.32 \mathrm{E}-05$ & $6.43 \mathrm{E}-06$ & core \\
\hline-29.8 & $2.32 E-05$ & $6.43 E-06$ & core \\
\hline-30.8 & 2.43E-05 & $6.74 \mathrm{E}-06$ & core \\
\hline-31.8 & 2.32E-05 & $6.43 \mathrm{E}-06$ & core \\
\hline-32.8 & $2.32 \mathrm{E}-05$ & $6.43 \mathrm{E}-06$ & core \\
\hline-33.8 & 2.34E-05 & 6.51E-06 & core \\
\hline-34.8 & 2.57E-05 & $7.14 \mathrm{E}-06$ & core \\
\hline-35.8 & $2.43 \mathrm{E}-05$ & $6.74 E-06$ & core \\
\hline-36.8 & 2.34E-05 & $6.51 \mathrm{E}-06$ & core \\
\hline-38.8 & $2.43 \mathrm{E}-05$ & $6.74 \mathrm{E}-06$ & core \\
\hline-39.8 & 2.57E-05 & $7.14 \mathrm{E}-06$ & core \\
\hline-40.8 & $2.43 E-05$ & $6.74 \mathrm{E}-06$ & core \\
\hline-43.8 & $2.32 E-05$ & $6.43 \mathrm{E}-06$ & core \\
\hline-44.8 & $2.43 E-05$ & $6.74 \mathrm{E}-06$ & core \\
\hline-48.8 & $2.86 E+00$ & 7.96E-01 & core \\
\hline-49.8 & $2.86 \mathrm{E}+00$ & $7.96 \mathrm{E}-01$ & core \\
\hline-50.8 & $4.55 E+00$ & $1.26 E+00$ & core \\
\hline-51.8 & $7.23 E+00$ & $2.01 E+00$ & core \\
\hline-52.8 & $1.15 E+01$ & $3.19 E+00$ & core \\
\hline-53.8 & $7.23 E+00$ & $2.01 \mathrm{E}+00$ & core \\
\hline-54.8 & $4.55 E+00$ & $1.26 E+00$ & core \\
\hline-55.8 & $1.15 E+01$ & $3.19 E+00$ & core \\
\hline-56.8 & $1.82 \mathrm{E}+01$ & $5.07 \mathrm{E}+00$ & core \\
\hline
\end{tabular}


Conductivity Data at P20

\begin{tabular}{|c|c|c|c|}
\hline $\begin{array}{c}\text { Elevation } \\
\text { (ft) }\end{array}$ & $\begin{array}{c}\text { Kh } \\
\text { (ft/day) }\end{array}$ & $\begin{array}{c}\mathrm{Kv} \\
\text { (ft/day) }\end{array}$ & $\begin{array}{c}\text { Data } \\
\text { Source }\end{array}$ \\
\hline-57.8 & $1.82 E+01$ & $5.07 E+00$ & core \\
\hline-58.8 & $1.80 \mathrm{E}+00$ & $5.01 \mathrm{E}-01$ & core \\
\hline-59.8 & $4.55 E+00$ & $1.26 \mathrm{E}+00$ & core \\
\hline-60.8 & $7.23 \mathrm{E}+00$ & $2.01 \mathrm{E}+00$ & core \\
\hline-61.8 & $7.23 E+00$ & $2.01 E+00$ & core \\
\hline-62.8 & $4.55 E+00$ & $1.26 \mathrm{E}+00$ & core \\
\hline-63.8 & $1.15 E+01$ & $3.19 E+00$ & core \\
\hline-64.8 & $1.82 E+01$ & $5.07 \mathrm{E}+00$ & core \\
\hline-65.8 & $1.82 E+01$ & $5.07 E+00$ & core \\
\hline-66.8 & $1.82 \mathrm{E}+01$ & $5.07 E+00$ & core \\
\hline-68.8 & $2.32 E-05$ & $6.43 E-06$ & core \\
\hline-71.8 & 2.32E-05 & 6.43E-06 & core \\
\hline-72.8 & $7.23 E+00$ & $2.01 \mathrm{E}+00$ & core \\
\hline-74.8 & $4.55 E+00$ & $1.26 \mathrm{E}+00$ & core \\
\hline-75.8 & $2.86 E+00$ & $7.96 \mathrm{E}-01$ & core \\
\hline-76.8 & $4.55 E+00$ & $1.26 \mathrm{E}+00$ & core \\
\hline-78.8 & $7.23 E+00$ & $2.01 E+00$ & core \\
\hline-79.8 & $1.15 E+01$ & $3.19 \mathrm{E}+00$ & core \\
\hline-80.8 & $1.82 \mathrm{E}+01$ & $5.07 E+00$ & core \\
\hline-81.8 & $1.15 E+01$ & $3.19 E+00$ & core \\
\hline-82.8 & $4.55 E+00$ & $1.26 E+00$ & core \\
\hline-83.8 & $1.15 E+01$ & $3.19 E+00$ & core \\
\hline-84.8 & $1.82 E+01$ & $5.07 E+00$ & core \\
\hline-85.8 & $1.15 E+01$ & $3.19 \mathrm{E}+00$ & core \\
\hline-86.8 & $1.15 E+01$ & $3.19 \mathrm{E}+00$ & core \\
\hline-87.8 & $1.82 E+01$ & $5.07 E+00$ & core \\
\hline-88.8 & $1.82 E+01$ & $5.07 \mathrm{E}+00$ & core \\
\hline-89.8 & $1.82 E+01$ & $5.07 \mathrm{E}+00$ & core \\
\hline-90.8 & $1.82 E+01$ & $5.07 \mathrm{E}+00$ & core \\
\hline-91.8 & $2.90 \mathrm{E}+01$ & $8.05 E+00$ & core \\
\hline-92.8 & $1.15 E+01$ & $3.19 E+00$ & core \\
\hline-93.8 & $1.15 E+01$ & $3.19 E+00$ & core \\
\hline-94.8 & $2.90 E+01$ & $8.05 \mathrm{E}+00$ & core \\
\hline-95.8 & $1.15 E+01$ & $3.19 E+00$ & core \\
\hline-96.8 & $2.90 \mathrm{E}+01$ & $8.05 E+00$ & core \\
\hline-97.8 & $2.90 E+01$ & $8.05 E+00$ & core \\
\hline-98.8 & $1.82 \mathrm{E}+01$ & $5.07 \mathrm{E}+00$ & core \\
\hline-99.8 & $1.82 E+01$ & $5.07 E+00$ & core \\
\hline-100.8 & $2.90 \mathrm{E}+01$ & $8.05 E+00$ & core \\
\hline-101.8 & $2.90 \mathrm{E}+01$ & $8.05 E+00$ & core \\
\hline-102.8 & $1.82 \mathrm{E}+01$ & $5.07 E+00$ & core \\
\hline-103.8 & $4.55 E+00$ & $1.26 \mathrm{E}+00$ & core \\
\hline-104.8 & $1.82 E+01$ & $5.07 E+00$ & core \\
\hline-105.8 & $4.55 E+00$ & $1.26 \mathrm{E}+00$ & core \\
\hline-106.8 & $2.86 \mathrm{E}+00$ & 7.96E-01 & core \\
\hline-107.8 & $1.15 \mathrm{E}+01$ & $3.19 E+00$ & core \\
\hline-108.8 & $1.82 \mathrm{E}+01$ & $5.07 E+00$ & core \\
\hline-109.8 & $1.15 E+01$ & $3.19 E+00$ & core \\
\hline
\end{tabular}


Conductivity Data at P20

\begin{tabular}{|c|c|c|c|}
\hline $\begin{array}{c}\text { Elevation } \\
\text { (ft) }\end{array}$ & $\begin{array}{c}\text { Kh } \\
\text { (ft/day) }\end{array}$ & $\begin{array}{c}\mathrm{Kv} \\
\text { (ft/day) }\end{array}$ & $\begin{array}{c}\text { Data } \\
\text { Source }\end{array}$ \\
\hline-110.8 & $1.82 E+01$ & $5.07 E+00$ & core \\
\hline-111.8 & $2.90 \mathrm{E}+01$ & $8.05 E+00$ & core \\
\hline-112.8 & $4.55 E+00$ & $1.26 \mathrm{E}+00$ & core \\
\hline-113.8 & $1.82 E+01$ & $5.07 \mathrm{E}+00$ & core \\
\hline-114.8 & $2.90 E+01$ & $8.05 E+00$ & core \\
\hline-115.8 & $2.90 \mathrm{E}+01$ & $8.05 E+00$ & core \\
\hline-116.8 & $1.15 E+01$ & $3.19 E+00$ & core \\
\hline-117.8 & $2.86 E+00$ & $7.96 \mathrm{E}-01$ & core \\
\hline-118.8 & $1.80 E+00$ & $5.01 E-01$ & core \\
\hline-119.8 & $1.80 E+00$ & 5.01E-01 & core \\
\hline-120.8 & $4.55 E+00$ & $1.26 E+00$ & core \\
\hline-121.8 & $1.15 E+01$ & $3.19 E+00$ & core \\
\hline-122.8 & $1.80 E+00$ & $5.01 \mathrm{E}-01$ & core \\
\hline-123.8 & $7.23 E+00$ & $2.01 \mathrm{E}+00$ & core \\
\hline-125.8 & $1.15 \mathrm{E}+01$ & $3.19 E+00$ & core \\
\hline-126.8 & $1.15 \mathrm{E}+01$ & $3.19 E+00$ & core \\
\hline-128.8 & $1.82 E+01$ & $5.07 E+00$ & core \\
\hline-129.8 & $4.55 \mathrm{E}+00$ & $1.26 E+00$ & core \\
\hline-130.8 & $1.82 \mathrm{E}+01$ & $5.07 E+00$ & core \\
\hline-131.8 & $1.82 E+01$ & $5.07 E+00$ & core \\
\hline-132.8 & $1.15 \mathrm{E}+01$ & $3.19 E+00$ & core \\
\hline-133.8 & $1.15 E+01$ & $3.19 E+00$ & core \\
\hline-135.8 & $4.55 E+00$ & $1.26 \mathrm{E}+00$ & core \\
\hline-136.8 & $2.86 E+00$ & $7.96 \mathrm{E}-01$ & core \\
\hline-137.8 & $2.86 E+00$ & $7.96 \mathrm{E}-01$ & core \\
\hline-138.8 & $7.23 E+00$ & $2.01 E+00$ & core \\
\hline-139.8 & $7.23 E+00$ & $2.01 \mathrm{E}+00$ & core \\
\hline-140.8 & $2.86 E+00$ & $7.96 \mathrm{E}-01$ & core \\
\hline-141.8 & $4.55 E+00$ & $1.26 \mathrm{E}+00$ & core \\
\hline-142.8 & $4.55 E+00$ & $1.26 E+00$ & core \\
\hline-143.8 & $4.55 E+00$ & $1.26 \mathrm{E}+00$ & core \\
\hline-144.8 & $1.82 E+01$ & $5.07 E+00$ & core \\
\hline-145.8 & $1.15 E+01$ & $3.19 E+00$ & core \\
\hline-146.8 & $1.15 E+01$ & $3.19 E+00$ & core \\
\hline-148.8 & $2.90 E+01$ & $8.05 E+00$ & core \\
\hline-149.8 & $2.90 E+01$ & $8.05 E+00$ & core \\
\hline-150.8 & $7.23 E+00$ & $2.01 E+00$ & core \\
\hline-151.8 & $1.21 \mathrm{E}-03$ & 3.37E-04 & core \\
\hline-153.8 & $1.82 E+01$ & $5.07 E+00$ & core \\
\hline-154.8 & $2.90 E+01$ & $8.05 E+00$ & core \\
\hline-155.8 & $1.82 E+01$ & $5.07 E+00$ & core \\
\hline-156.8 & $1.82 E+01$ & $5.07 E+00$ & core \\
\hline-158.8 & $2.86 E+00$ & 7.96E-01 & core \\
\hline-159.8 & $2.32 E-05$ & $6.43 \mathrm{E}-06$ & core \\
\hline-160.8 & $2.86 E+00$ & $7.96 E-01$ & core \\
\hline
\end{tabular}


Conductivity Data at RBW1

\begin{tabular}{|c|c|c|c|}
\hline $\begin{array}{c}\text { Elevation } \\
\text { (ft) }\end{array}$ & $\begin{array}{c}\text { Kh } \\
\text { (ft/day) }\end{array}$ & $\begin{array}{c}\text { Kv } \\
\text { (ft/day) }\end{array}$ & $\begin{array}{c}\text { Data } \\
\text { Source }\end{array}$ \\
\hline 283.5 & $3.28 \mathrm{E}+00$ & $9.10 \mathrm{E}-01$ & $\mathrm{cpt}$ \\
\hline 282.5 & $4.91 \mathrm{E}+00$ & $1.37 \mathrm{E}+00$ & $\mathrm{cpt}$ \\
\hline 281.5 & $6.52 \mathrm{E}+00$ & $1.81 \mathrm{E}+00$ & $\mathrm{cpt}$ \\
\hline 280.5 & $5.58 \mathrm{E}+00$ & $1.55 \mathrm{E}+00$ & $\mathrm{cpt}$ \\
\hline 279.5 & $5.11 \mathrm{E}+00$ & $1.42 \mathrm{E}+00$ & $\mathrm{cpt}$ \\
\hline 278.5 & $2.62 \mathrm{E}-01$ & $7.28 \mathrm{E}-02$ & $\mathrm{cpt}$ \\
\hline 277.5 & $1.50 \mathrm{E}-02$ & $4.16 \mathrm{E}-03$ & $\mathrm{cpt}$ \\
\hline 276.5 & $3.93 \mathrm{E}+00$ & $1.09 \mathrm{E}+00$ & $\mathrm{cpt}$ \\
\hline 275.5 & $9.59 \mathrm{E}-03$ & $2.66 \mathrm{E}-03$ & $\mathrm{cpt}$ \\
\hline 274.5 & $9.04 \mathrm{E}+00$ & $2.51 \mathrm{E}+00$ & $\mathrm{cpt}$ \\
\hline 273.5 & $7.33 \mathrm{E}+00$ & $2.04 \mathrm{E}+00$ & $\mathrm{cpt}$ \\
\hline 272.5 & $6.93 \mathrm{E}+00$ & $1.93 \mathrm{E}+00$ & $\mathrm{cpt}$ \\
\hline 271.5 & $1.41 \mathrm{E}+01$ & $3.90 \mathrm{E}+00$ & $\mathrm{cpt}$ \\
\hline 270.5 & $1.24 \mathrm{E}+01$ & $3.44 \mathrm{E}+00$ & $\mathrm{cpt}$ \\
\hline 269.5 & $9.57 \mathrm{E}+00$ & $2.66 \mathrm{E}+00$ & $\mathrm{cpt}$ \\
\hline 268.5 & $2.09 \mathrm{E}-01$ & $5.79 \mathrm{E}-02$ & $\mathrm{cpt}$ \\
\hline 267.5 & $1.05 \mathrm{E}+01$ & $2.91 \mathrm{E}+00$ & $\mathrm{cpt}$ \\
\hline 266.5 & $9.33 \mathrm{E}+00$ & $2.59 \mathrm{E}+00$ & $\mathrm{cpt}$ \\
\hline 265.5 & $7.99 \mathrm{E}+00$ & $2.22 \mathrm{E}+00$ & $\mathrm{cpt}$ \\
\hline 264.5 & $8.50 \mathrm{E}+00$ & $2.36 \mathrm{E}+00$ & $\mathrm{cpt}$ \\
\hline 263.5 & $8.92 \mathrm{E}+00$ & $2.48 \mathrm{E}+00$ & $\mathrm{cpt}$ \\
\hline 262.5 & $7.98 \mathrm{E}+00$ & $2.22 \mathrm{E}+00$ & $\mathrm{cpt}$ \\
\hline 261.5 & $7.25 \mathrm{E}+00$ & $2.01 \mathrm{E}+00$ & $\mathrm{cpt}$ \\
\hline 260.5 & $7.64 \mathrm{E}+00$ & $2.12 \mathrm{E}+00$ & $\mathrm{cpt}$ \\
\hline 259.5 & $9.08 \mathrm{E}+00$ & $2.52 \mathrm{E}+00$ & $\mathrm{cpt}$ \\
\hline 258.5 & $9.25 \mathrm{E}+00$ & $2.57 \mathrm{E}+00$ & $\mathrm{cpt}$ \\
\hline 257.5 & $9.40 \mathrm{E}+00$ & $2.61 \mathrm{E}+00$ & $\mathrm{cpt}$ \\
\hline 256.5 & $7.03 \mathrm{E}+00$ & $1.95 \mathrm{E}+00$ & $\mathrm{cpt}$ \\
\hline 255.5 & $5.55 \mathrm{E}+00$ & $1.54 \mathrm{E}+00$ & $\mathrm{cpt}$ \\
\hline 254.5 & $7.29 \mathrm{E}+00$ & $2.03 \mathrm{E}+00$ & $\mathrm{cpt}$ \\
\hline 253.5 & $7.13 \mathrm{E}+00$ & $1.98 \mathrm{E}+00$ & $\mathrm{cpt}$ \\
\hline 252.5 & $7.54 \mathrm{E}+00$ & $2.09 \mathrm{E}+00$ & $\mathrm{cpt}$ \\
\hline 251.5 & $8.45 \mathrm{E}+00$ & $2.35 \mathrm{E}+00$ & $\mathrm{cpt}$ \\
\hline 250.5 & $9.82 \mathrm{E}+00$ & $2.73 \mathrm{E}+00$ & $\mathrm{cpt}$ \\
\hline 249.5 & $9.68 \mathrm{E}+00$ & $2.69 \mathrm{E}+00$ & $\mathrm{cpt}$ \\
\hline 248.5 & $9.00 \mathrm{E}+00$ & $2.50 \mathrm{E}+00$ & $\mathrm{cpt}$ \\
\hline 247.5 & $8.71 \mathrm{E}+00$ & $2.42 \mathrm{E}+00$ & $\mathrm{cpt}$ \\
\hline 246.5 & $5.89 \mathrm{E}+00$ & $1.64 \mathrm{E}+00$ & $\mathrm{cpt}$ \\
\hline 245.5 & $3.16 \mathrm{E}+00$ & $8.78 \mathrm{E}-01$ & $\mathrm{cpt}$ \\
\hline 244.5 & $5.48 \mathrm{E}+00$ & $1.52 \mathrm{E}+00$ & $\mathrm{cpt}$ \\
\hline 243.5 & $7.30 \mathrm{E}+00$ & $2.03 \mathrm{E}+00$ & $\mathrm{cpt}$ \\
\hline 242.5 & $8.19 \mathrm{E}+00$ & $2.28 \mathrm{E}+00$ & $\mathrm{cpt}$ \\
\hline 241.5 & $6.53 \mathrm{E}+00$ & $1.81 \mathrm{E}+00$ & $\mathrm{cpt}$ \\
\hline 240.5 & $5.82 \mathrm{E}+00$ & $1.62 \mathrm{E}+00$ & $\mathrm{cpt}$ \\
\hline 239.5 & $1.04 \mathrm{E}-03$ & $2.90 \mathrm{E}-04$ & $\mathrm{cpt}$ \\
\hline 238.5 & $6.04 \mathrm{E}-03$ & $1.68 \mathrm{E}-03$ & $\mathrm{cpt}$ \\
\hline 236.5 & $6.77 \mathrm{E}-01$ & $1.88 \mathrm{E}-01$ & $\mathrm{cpt}$ \\
\hline & $1.44 \mathrm{E}+01$ & $4.00 \mathrm{E}+00$ & $\mathrm{cpt}$ \\
\hline & & & \\
\hline
\end{tabular}


Conductivity Data at RBW1

\begin{tabular}{|c|c|c|c|}
\hline $\begin{array}{c}\text { Elevation } \\
\text { (ft) }\end{array}$ & $\begin{array}{c}\text { Kh } \\
\text { (ft/day) }\end{array}$ & $\begin{array}{c}\text { Kv } \\
\text { (ft/day) }\end{array}$ & $\begin{array}{c}\text { Data } \\
\text { Source }\end{array}$ \\
\hline 235.5 & $1.45 E+01$ & $4.04 \mathrm{E}+00$ & $\mathrm{cpt}$ \\
\hline 234.5 & $1.53 \mathrm{E}+01$ & $4.24 \mathrm{E}+00$ & $\mathrm{cpt}$ \\
\hline 233.5 & $2.36 \mathrm{E}+01$ & $6.55 \mathrm{E}+00$ & cpt \\
\hline 232.5 & $3.35 E+01$ & $9.30 \mathrm{E}+00$ & $\mathrm{cpt}$ \\
\hline 231.5 & $3.68 E+01$ & $1.02 E+01$ & $\mathrm{cpt}$ \\
\hline 230.5 & $3.06 \mathrm{E}+01$ & $8.51 \mathrm{E}+00$ & cpt \\
\hline 229.5 & $2.84 E+01$ & $7.89 \mathrm{E}+00$ & cpt \\
\hline 228.5 & $3.67 E+01$ & $1.02 E+01$ & $\mathrm{cpt}$ \\
\hline 227.5 & $4.92 \mathrm{E}+01$ & $1.37 E+01$ & $\mathrm{cpt}$ \\
\hline 226.5 & $5.76 \mathrm{E}+01$ & $1.60 \mathrm{E}+01$ & $\mathrm{cpt}$ \\
\hline 225.5 & $6.23 E+01$ & $1.73 \mathrm{E}+01$ & cpt \\
\hline 224.5 & $5.12 \mathrm{E}+01$ & $1.42 \mathrm{E}+01$ & cpt \\
\hline 223.5 & $4.62 E+01$ & $1.29 \mathrm{E}+01$ & cpt \\
\hline 222.5 & $7.27 E+01$ & $2.02 \mathrm{E}+01$ & cpt \\
\hline 221.5 & $1.00 \mathrm{E}+02$ & $2.78 \mathrm{E}+01$ & $\mathrm{cpt}$ \\
\hline 220.5 & $9.60 \mathrm{E}+01$ & $2.67 \mathrm{E}+01$ & $\mathrm{cpt}$ \\
\hline 219.5 & $1.07 E+00$ & $2.98 \mathrm{E}-01$ & cpt \\
\hline 218.5 & $7.70 E-04$ & $2.10 \mathrm{E}-04$ & $\mathrm{cpt}$ \\
\hline 217.5 & $3.68 \mathrm{E}-01$ & $1.02 \mathrm{E}-01$ & $\mathrm{cpt}$ \\
\hline 216.5 & $2.95 \mathrm{E}+01$ & $8.21 E+00$ & $\mathrm{cpt}$ \\
\hline 215.5 & $4.13 E+01$ & $1.15 \mathrm{E}+01$ & $\mathrm{cpt}$ \\
\hline 214.5 & $3.77 E+01$ & $1.05 \mathrm{E}+01$ & $\mathrm{cpt}$ \\
\hline 213.5 & $3.97 \mathrm{E}+01$ & $1.10 \mathrm{E}+01$ & $\mathrm{cpt}$ \\
\hline 212.5 & $5.59 \mathrm{E}+01$ & $1.55 \mathrm{E}+01$ & $\mathrm{cpt}$ \\
\hline 211.5 & $1.00 \mathrm{E}+02$ & $2.78 \mathrm{E}+01$ & $\mathrm{cpt}$ \\
\hline 210.5 & $7.12 \mathrm{E}+01$ & $1.98 \mathrm{E}+01$ & $\mathrm{cpt}$ \\
\hline 209.5 & $9.20 \mathrm{E}-04$ & $2.60 \mathrm{E}-04$ & cpt \\
\hline 208.5 & $5.41 E+01$ & $1.50 \mathrm{E}+01$ & cpt \\
\hline 207.5 & $2.99 E+01$ & $8.30 \mathrm{E}+00$ & $\mathrm{cpt}$ \\
\hline 206.5 & $4.36 \mathrm{E}+01$ & $1.21 \mathrm{E}+01$ & $\mathrm{cpt}$ \\
\hline 205.5 & $1.33 \mathrm{E}-03$ & $3.70 \mathrm{E}-04$ & $\mathrm{cpt}$ \\
\hline 204.5 & $4.00 \mathrm{E}+01$ & $1.11 \mathrm{E}+01$ & cpt \\
\hline 203.5 & $5.51 \mathrm{E}+01$ & $1.53 \mathrm{E}+01$ & $\mathrm{cpt}$ \\
\hline 202.5 & $5.10 \mathrm{E}+01$ & $1.42 \mathrm{E}+01$ & $\mathrm{cpt}$ \\
\hline 201.5 & $8.99 \mathrm{E}+00$ & $2.50 \mathrm{E}+00$ & $\mathrm{cpt}$ \\
\hline 200.5 & 1.87E-01 & $5.19 \mathrm{E}-02$ & $\mathrm{cpt}$ \\
\hline 199.5 & 4.92E-01 & 1.37E-01 & cpt \\
\hline 198.5 & $6.60 \mathrm{E}-01$ & $1.83 \mathrm{E}-01$ & $\mathrm{cpt}$ \\
\hline 197.5 & $1.70 \mathrm{E}-01$ & 4.71E-02 & cpt \\
\hline 196.5 & $5.51 \mathrm{E}-02$ & 1.53E-02 & $\mathrm{cpt}$ \\
\hline 195.5 & 1.17E-01 & $3.25 \mathrm{E}-02$ & $\mathrm{cpt}$ \\
\hline 194.5 & $4.40 \mathrm{E}-01$ & $1.22 \mathrm{E}-01$ & $\mathrm{cpt}$ \\
\hline 193.5 & $4.31 E+00$ & $1.20 \mathrm{E}+00$ & cpt \\
\hline 192.5 & $7.77 \mathrm{E}+00$ & $2.16 \mathrm{E}+00$ & $\mathrm{cpt}$ \\
\hline 191.5 & $3.92 \mathrm{E}+00$ & $1.09 \mathrm{E}+00$ & $\mathrm{cpt}$ \\
\hline 190.5 & $8.23 E+00$ & $2.29 \mathrm{E}+00$ & cpt \\
\hline 189.5 & $8.94 \mathrm{E}+00$ & $2.48 \mathrm{E}+00$ & cpt \\
\hline 188.5 & $9.76 \mathrm{E}+01$ & $2.71 E+01$ & cpt \\
\hline
\end{tabular}


Conductivity Data at RBW1

\begin{tabular}{|c|c|c|c|}
\hline $\begin{array}{c}\text { Elevation } \\
\text { (ft) }\end{array}$ & $\begin{array}{c}\mathbf{K h} \\
\text { (ft/day) }\end{array}$ & $\begin{array}{c}\mathrm{Kv} \\
\text { (ft/day) }\end{array}$ & $\begin{array}{c}\text { Data } \\
\text { Source }\end{array}$ \\
\hline 187.5 & $2.58 \mathrm{E}+01$ & $7.16 \mathrm{E}+00$ & $\mathrm{cpt}$ \\
\hline 186.5 & $2.85 \mathrm{E}+01$ & $7.90 \mathrm{E}+00$ & $\mathrm{cpt}$ \\
\hline 185.5 & $1.96 \mathrm{E}+01$ & $5.46 \mathrm{E}+00$ & $\mathrm{cpt}$ \\
\hline 184.5 & $3.83 \mathrm{E}+01$ & $1.07 \mathrm{E}+01$ & $\mathrm{cpt}$ \\
\hline 183.5 & $4.97 \mathrm{E}+01$ & $1.38 \mathrm{E}+01$ & $\mathrm{cpt}$ \\
\hline 182.5 & $6.47 \mathrm{E}+01$ & $1.80 \mathrm{E}+01$ & $\mathrm{cpt}$ \\
\hline 181.5 & $6.10 \mathrm{E}+01$ & $1.70 \mathrm{E}+01$ & $\mathrm{cpt}$ \\
\hline 180.5 & $6.02 \mathrm{E}+01$ & $1.67 \mathrm{E}+01$ & $\mathrm{cpt}$ \\
\hline 179.5 & $7.44 \mathrm{E}+01$ & $2.07 \mathrm{E}+01$ & $\mathrm{cpt}$ \\
\hline 178.5 & $7.80 \mathrm{E}+01$ & $2.17 \mathrm{E}+01$ & $\mathrm{cpt}$ \\
\hline 177.5 & $1.00 \mathrm{E}+02$ & $2.78 \mathrm{E}+01$ & $\mathrm{cpt}$ \\
\hline 176.5 & $9.70 \mathrm{E}+01$ & $2.69 \mathrm{E}+01$ & $\mathrm{cpt}$ \\
\hline 175.5 & $8.18 \mathrm{E}+01$ & $2.27 \mathrm{E}+01$ & $\mathrm{cpt}$ \\
\hline 174.5 & $5.22 \mathrm{E}+01$ & $1.45 \mathrm{E}+01$ & $\mathrm{cpt}$ \\
\hline 173.5 & $1.54 \mathrm{E}+01$ & $4.27 \mathrm{E}+00$ & $\mathrm{cpt}$ \\
\hline
\end{tabular}


Conductivity Data at RBW2CL

\begin{tabular}{|c|c|c|c|}
\hline $\begin{array}{c}\text { Elevation } \\
\text { (ft) }\end{array}$ & $\begin{array}{c}\mathbf{K h} \\
\text { (ft/day) }\end{array}$ & $\begin{array}{c}\text { Kv } \\
\text { (ft/day) }\end{array}$ & $\begin{array}{c}\text { Data } \\
\text { Source }\end{array}$ \\
\hline 324.6 & $3.48 \mathrm{E}+00$ & $9.66 \mathrm{E}-01$ & $\mathrm{cpt}$ \\
\hline 323.6 & $4.08 \mathrm{E}+00$ & $1.13 \mathrm{E}+00$ & $\mathrm{cpt}$ \\
\hline 322.6 & $4.49 \mathrm{E}+00$ & $1.25 \mathrm{E}+00$ & $\mathrm{cpt}$ \\
\hline 321.6 & $9.92 \mathrm{E}-03$ & $2.76 \mathrm{E}-03$ & $\mathrm{cpt}$ \\
\hline 320.6 & $9.06 \mathrm{E}-03$ & $2.52 \mathrm{E}-03$ & $\mathrm{cpt}$ \\
\hline 319.6 & $7.81 \mathrm{E}-03$ & $2.17 \mathrm{E}-03$ & $\mathrm{cpt}$ \\
\hline 318.6 & $6.39 \mathrm{E}-01$ & $1.77 \mathrm{E}-01$ & $\mathrm{cpt}$ \\
\hline 317.6 & $1.76 \mathrm{E}-02$ & $4.90 \mathrm{E}-03$ & $\mathrm{cpt}$ \\
\hline 316.6 & $1.87 \mathrm{E}-02$ & $5.18 \mathrm{E}-03$ & $\mathrm{cpt}$ \\
\hline 315.6 & $6.60 \mathrm{E}-03$ & $1.83 \mathrm{E}-03$ & $\mathrm{cpt}$ \\
\hline 314.6 & $2.93 \mathrm{E}-03$ & $8.10 \mathrm{E}-04$ & $\mathrm{cpt}$ \\
\hline 313.6 & $2.45 \mathrm{E}-03$ & $6.80 \mathrm{E}-04$ & $\mathrm{cpt}$ \\
\hline 312.6 & $2.88 \mathrm{E}-03$ & $8.00 \mathrm{E}-04$ & $\mathrm{cpt}$ \\
\hline 311.6 & $2.31 \mathrm{E}-03$ & $6.40 \mathrm{E}-04$ & $\mathrm{cpt}$ \\
\hline 310.6 & $1.12 \mathrm{E}-01$ & $3.10 \mathrm{E}-02$ & $\mathrm{cpt}$ \\
\hline 309.6 & $8.70 \mathrm{E}-01$ & $2.42 \mathrm{E}-01$ & $\mathrm{cpt}$ \\
\hline 308.6 & $2.27 \mathrm{E}-01$ & $6.30 \mathrm{E}-02$ & $\mathrm{cpt}$ \\
\hline 307.6 & $8.78 \mathrm{E}+00$ & $2.44 \mathrm{E}+00$ & $\mathrm{cpt}$ \\
\hline 306.6 & $6.53 \mathrm{E}-03$ & $1.81 \mathrm{E}-03$ & $\mathrm{cpt}$ \\
\hline 305.6 & $3.67 \mathrm{E}-02$ & $1.02 \mathrm{E}-02$ & $\mathrm{cpt}$ \\
\hline 304.6 & $8.30 \mathrm{E}+00$ & $2.30 \mathrm{E}+00$ & $\mathrm{cpt}$ \\
\hline 303.6 & $1.00 \mathrm{E}+02$ & $2.78 \mathrm{E}+01$ & $\mathrm{cpt}$ \\
\hline 302.6 & $2.21 \mathrm{E}+01$ & $6.14 \mathrm{E}+00$ & $\mathrm{cpt}$ \\
\hline 301.6 & $2.24 \mathrm{E}+00$ & $6.22 \mathrm{E}-01$ & $\mathrm{cpt}$ \\
\hline 300.6 & $1.15 \mathrm{E}-03$ & $3.20 \mathrm{E}-04$ & $\mathrm{cpt}$ \\
\hline 299.6 & $9.06 \mathrm{E}+00$ & $2.52 \mathrm{E}+00$ & $\mathrm{cpt}$ \\
\hline 298.6 & $4.50 \mathrm{E}-01$ & $1.25 \mathrm{E}-01$ & $\mathrm{cpt}$ \\
\hline 297.6 & $8.50 \mathrm{E}-04$ & $2.40 \mathrm{E}-04$ & $\mathrm{cpt}$ \\
\hline 296.6 & $6.06 \mathrm{E}+00$ & $1.68 \mathrm{E}+00$ & $\mathrm{cpt}$ \\
\hline 295.6 & $6.12 \mathrm{E}+00$ & $1.70 \mathrm{E}+00$ & $\mathrm{cpt}$ \\
\hline 294.6 & $3.72 \mathrm{E}+00$ & $1.03 \mathrm{E}+00$ & $\mathrm{cpt}$ \\
\hline 293.6 & $1.04 \mathrm{E}+01$ & $2.88 \mathrm{E}+00$ & $\mathrm{cpt}$ \\
\hline 292.6 & $8.90 \mathrm{E}+00$ & $2.47 \mathrm{E}+00$ & $\mathrm{cpt}$ \\
\hline 291.6 & $6.75 \mathrm{E}+00$ & $1.87 \mathrm{E}+00$ & $\mathrm{cpt}$ \\
\hline 290.6 & $7.13 \mathrm{E}+00$ & $1.98 \mathrm{E}+00$ & $\mathrm{cpt}$ \\
\hline 289.6 & $1.68 \mathrm{E}+00$ & $4.67 \mathrm{E}-01$ & $\mathrm{cpt}$ \\
\hline 288.6 & $2.00 \mathrm{E}-01$ & $5.55 \mathrm{E}-02$ & $\mathrm{cpt}$ \\
\hline 287.6 & $2.40 \mathrm{E}-03$ & $6.70 \mathrm{E}-04$ & $\mathrm{cpt}$ \\
\hline 286.6 & $3.03 \mathrm{E}-01$ & $8.40 \mathrm{E}-02$ & $\mathrm{cpt}$ \\
\hline 285.6 & $1.25 \mathrm{E}-03$ & $3.50 \mathrm{E}-04$ & $\mathrm{cpt}$ \\
\hline 284.6 & $5.91 \mathrm{E}+00$ & $1.64 \mathrm{E}+00$ & $\mathrm{cpt}$ \\
\hline 283.6 & $6.23 \mathrm{E}+00$ & $1.73 \mathrm{E}+00$ & $\mathrm{cpt}$ \\
\hline 282.6 & $7.00 \mathrm{E}+00$ & $1.95 \mathrm{E}+00$ & $\mathrm{cpt}$ \\
\hline 281.6 & $6.25 \mathrm{E}+00$ & $1.74 \mathrm{E}+00$ & $\mathrm{cpt}$ \\
\hline 280.6 & $6.21 \mathrm{E}+00$ & $1.73 \mathrm{E}+00$ & $\mathrm{cpt}$ \\
\hline 278.6 & $9.50 \mathrm{E}-04$ & $2.60 \mathrm{E}-04$ & $\mathrm{cpt}$ \\
\hline 277.6 & $1.62 \mathrm{E}-03$ & $4.50 \mathrm{E}-04$ & $\mathrm{cpt}$ \\
\hline & $1.07 \mathrm{E}-03$ & $3.00 \mathrm{E}-04$ & $\mathrm{cpt}$ \\
\hline
\end{tabular}


Conductivity Data at RBW2CL

\begin{tabular}{|c|c|c|c|}
\hline $\begin{array}{c}\text { Elevation } \\
\text { (ft) }\end{array}$ & $\begin{array}{c}\text { Kh } \\
\text { (ft/day) }\end{array}$ & $\begin{array}{c}\text { Kv } \\
\text { (ft/day) }\end{array}$ & $\begin{array}{c}\text { Data } \\
\text { Source }\end{array}$ \\
\hline 276.6 & $1.30 \mathrm{E}-02$ & $3.60 \mathrm{E}-03$ & $\mathrm{cpt}$ \\
\hline 275.6 & $5.97 \mathrm{E}-03$ & $1.66 \mathrm{E}-03$ & $\mathrm{cpt}$ \\
\hline 274.6 & $1.37 \mathrm{E}-03$ & $3.80 \mathrm{E}-04$ & $\mathrm{cpt}$ \\
\hline 273.6 & $1.89 \mathrm{E}-02$ & $5.25 \mathrm{E}-03$ & $\mathrm{cpt}$ \\
\hline 272.6 & $7.00 \mathrm{E}+00$ & $1.94 \mathrm{E}+00$ & $\mathrm{cpt}$ \\
\hline 271.6 & $7.92 \mathrm{E}+00$ & $2.20 \mathrm{E}+00$ & $\mathrm{cpt}$ \\
\hline 270.6 & $7.71 \mathrm{E}+00$ & $2.14 \mathrm{E}+00$ & $\mathrm{cpt}$ \\
\hline 269.6 & $1.32 \mathrm{E}-01$ & $3.65 \mathrm{E}-02$ & $\mathrm{cpt}$ \\
\hline 268.6 & $1.56 \mathrm{E}-03$ & $4.30 \mathrm{E}-04$ & $\mathrm{cpt}$ \\
\hline 267.6 & $5.31 \mathrm{E}+00$ & $1.47 \mathrm{E}+00$ & $\mathrm{cpt}$ \\
\hline 266.6 & $7.69 \mathrm{E}+00$ & $2.14 \mathrm{E}+00$ & $\mathrm{cpt}$ \\
\hline 265.6 & $5.06 \mathrm{E}+00$ & $1.41 \mathrm{E}+00$ & $\mathrm{cpt}$ \\
\hline 264.6 & $8.08 \mathrm{E}-01$ & $2.24 \mathrm{E}-01$ & $\mathrm{cpt}$ \\
\hline 263.6 & $4.42 \mathrm{E}+00$ & $1.23 \mathrm{E}+00$ & $\mathrm{cpt}$ \\
\hline 262.6 & $6.50 \mathrm{E}+00$ & $1.81 \mathrm{E}+00$ & $\mathrm{cpt}$ \\
\hline 261.6 & $7.22 \mathrm{E}+00$ & $2.01 \mathrm{E}+00$ & $\mathrm{cpt}$ \\
\hline 260.6 & $7.47 \mathrm{E}+00$ & $2.08 \mathrm{E}+00$ & $\mathrm{cpt}$ \\
\hline 259.6 & $6.55 \mathrm{E}+00$ & $1.82 \mathrm{E}+00$ & $\mathrm{cpt}$ \\
\hline 258.6 & $5.77 \mathrm{E}+00$ & $1.60 \mathrm{E}+00$ & $\mathrm{cpt}$ \\
\hline 257.6 & $6.34 \mathrm{E}+00$ & $1.76 \mathrm{E}+00$ & $\mathrm{cpt}$ \\
\hline 256.6 & $5.49 \mathrm{E}+00$ & $1.53 \mathrm{E}+00$ & $\mathrm{cpt}$ \\
\hline 255.6 & $6.38 \mathrm{E}+00$ & $1.77 \mathrm{E}+00$ & $\mathrm{cpt}$ \\
\hline 254.6 & $5.58 \mathrm{E}+00$ & $1.55 \mathrm{E}+00$ & $\mathrm{cpt}$ \\
\hline 253.6 & $9.21 \mathrm{E}-01$ & $2.56 \mathrm{E}-01$ & $\mathrm{cpt}$ \\
\hline 252.6 & $3.65 \mathrm{E}-01$ & $1.02 \mathrm{E}-01$ & $\mathrm{cpt}$ \\
\hline 251.6 & $1.15 \mathrm{E}-03$ & $3.20 \mathrm{E}-04$ & $\mathrm{cpt}$ \\
\hline 250.6 & $2.13 \mathrm{E}+00$ & $5.90 \mathrm{E}-01$ & $\mathrm{cpt}$ \\
\hline 249.6 & $5.09 \mathrm{E}+01$ & $1.41 \mathrm{E}+01$ & $\mathrm{cpt}$ \\
\hline 248.6 & $1.98 \mathrm{E}-02$ & $5.51 \mathrm{E}-03$ & $\mathrm{cpt}$ \\
\hline 247.6 & $2.94 \mathrm{E}-02$ & $8.16 \mathrm{E}-03$ & $\mathrm{cpt}$ \\
\hline 246.6 & $8.88 \mathrm{E}+00$ & $2.47 \mathrm{E}+00$ & $\mathrm{cpt}$ \\
\hline 245.6 & $1.29 \mathrm{E}+00$ & $3.57 \mathrm{E}-01$ & $\mathrm{cpt}$ \\
\hline 244.6 & $1.98 \mathrm{E}+00$ & $5.49 \mathrm{E}-01$ & $\mathrm{cpt}$ \\
\hline 243.6 & $9.83 \mathrm{E}+00$ & $2.73 \mathrm{E}+00$ & $\mathrm{cpt}$ \\
\hline 242.6 & $6.86 \mathrm{E}+00$ & $1.90 \mathrm{E}+00$ & $\mathrm{cpt}$ \\
\hline 241.6 & $6.91 \mathrm{E}+00$ & $1.92 \mathrm{E}+00$ & $\mathrm{cpt}$ \\
\hline 240.6 & $3.26 \mathrm{E}+00$ & $9.07 \mathrm{E}-01$ & $\mathrm{cpt}$ \\
\hline 239.6 & $2.30 \mathrm{E}-01$ & $6.39 \mathrm{E}-02$ & $\mathrm{cpt}$ \\
\hline 238.6 & $6.60 \mathrm{E}-02$ & $1.83 \mathrm{E}-02$ & $\mathrm{cpt}$ \\
\hline 237.6 & $1.79 \mathrm{E}+01$ & $4.96 \mathrm{E}+00$ & $\mathrm{cpt}$ \\
\hline 236.6 & $1.79 \mathrm{E}+01$ & $4.98 \mathrm{E}+00$ & $\mathrm{cpt}$ \\
\hline 235.6 & $1.76 \mathrm{E}+01$ & $4.88 \mathrm{E}+00$ & $\mathrm{cpt}$ \\
\hline 234.6 & $5.29 \mathrm{E}+00$ & $1.47 \mathrm{E}+00$ & $\mathrm{cpt}$ \\
\hline 233.6 & $1.42 \mathrm{E}+01$ & $3.95 \mathrm{E}+00$ & $\mathrm{cpt}$ \\
\hline 232.6 & $1.76 \mathrm{E}+01$ & $4.89 \mathrm{E}+00$ & $\mathrm{cpt}$ \\
\hline 231.6 & $2.02 \mathrm{E}+01$ & $5.61 \mathrm{E}+00$ & $\mathrm{cpt}$ \\
\hline 230.6 & $2.43 \mathrm{E}+01$ & $6.75 \mathrm{E}+00$ & $\mathrm{cpt}$ \\
\hline 229.6 & $1.21 \mathrm{E}+01$ & $3.37 \mathrm{E}+00$ & $\mathrm{cpt}$ \\
\hline & & & \\
\hline
\end{tabular}


Conductivity Data at RBW2CL

\begin{tabular}{|c|c|c|c|}
\hline $\begin{array}{c}\text { Elevation } \\
\text { (ft) }\end{array}$ & $\begin{array}{c}\text { Kh } \\
\text { (ft/day) }\end{array}$ & $\begin{array}{c}\mathrm{Kv} \\
\text { (ft/day) }\end{array}$ & $\begin{array}{c}\text { Data } \\
\text { Source }\end{array}$ \\
\hline 228.6 & $9.96 E+00$ & $2.77 E+00$ & cpt \\
\hline 227.6 & $1.99 E+00$ & $5.52 \mathrm{E}-01$ & $\mathrm{cpt}$ \\
\hline 226.6 & $7.06 E+00$ & $1.96 \mathrm{E}+00$ & $\mathrm{cpt}$ \\
\hline 225.6 & 1.46E-01 & 4.07E-02 & $\mathrm{cpt}$ \\
\hline 224.6 & $4.44 E+01$ & $1.23 E+01$ & cpt \\
\hline 223.6 & $4.57 E+01$ & $1.27 E+01$ & $\mathrm{cpt}$ \\
\hline 222.6 & $5.22 E+01$ & $1.45 \mathrm{E}+01$ & cpt \\
\hline 221.6 & $5.90 \mathrm{E}+01$ & $1.64 E+01$ & cpt \\
\hline 220.6 & $5.67 E+01$ & $1.58 \mathrm{E}+01$ & $\mathrm{cpt}$ \\
\hline 219.6 & $4.31 \mathrm{E}+01$ & $1.20 \mathrm{E}+01$ & $\mathrm{cpt}$ \\
\hline 218.6 & $3.66 \mathrm{E}+01$ & $1.02 E+01$ & cpt \\
\hline 217.6 & $3.91 E+01$ & $1.09 \mathrm{E}+01$ & cpt \\
\hline 216.6 & $2.98 E+01$ & $8.28 E+00$ & cpt \\
\hline 215.6 & $5.28 E+01$ & $1.47 E+01$ & cpt \\
\hline 214.6 & $5.47 E+01$ & $1.52 E+01$ & cpt \\
\hline 213.6 & $2.03 E+01$ & $5.65 E+00$ & cpt \\
\hline 212.6 & $1.68 E+01$ & $4.68 E+00$ & $\mathrm{cpt}$ \\
\hline 211.6 & $4.48 E+01$ & $1.25 \mathrm{E}+01$ & cpt \\
\hline 210.6 & $4.88 E+01$ & $1.36 \mathrm{E}+01$ & $\mathrm{cpt}$ \\
\hline 209.6 & $4.54 E+01$ & $1.26 \mathrm{E}+01$ & cpt \\
\hline 208.6 & $4.61 E+01$ & $1.28 \mathrm{E}+01$ & $\mathrm{cpt}$ \\
\hline 207.6 & $6.68 E+01$ & $1.86 E+01$ & $\mathrm{cpt}$ \\
\hline 206.6 & $1.00 E+02$ & $2.78 \mathrm{E}+01$ & cpt \\
\hline 205.6 & $1.00 E+02$ & $2.78 \mathrm{E}+01$ & $\mathrm{cpt}$ \\
\hline 201.6 & $1.00 E+02$ & $2.78 E+01$ & $\mathrm{cpt}$ \\
\hline 200.6 & $1.00 E+02$ & $2.78 E+01$ & cpt \\
\hline 195.6 & $1.00 E+02$ & $2.78 E+01$ & cpt \\
\hline 192.6 & $1.00 E+02$ & $2.78 E+01$ & cpt \\
\hline 191.6 & $1.00 E+02$ & $2.78 E+01$ & $\mathrm{cpt}$ \\
\hline 187.6 & $1.01 \mathrm{E}-03$ & $2.80 \mathrm{E}-04$ & cpt \\
\hline 186.6 & $3.67 E-03$ & $1.02 E-03$ & cpt \\
\hline 185.6 & $4.50 \mathrm{E}-03$ & $1.25 \mathrm{E}-03$ & $\mathrm{cpt}$ \\
\hline 181.6 & $3.86 E+01$ & $1.07 E+01$ & cpt \\
\hline 180.6 & $6.87 E+01$ & $1.91 E+01$ & $\mathrm{cpt}$ \\
\hline 178.6 & $1.00 E+02$ & $2.78 \mathrm{E}+01$ & cpt \\
\hline 164.8 & $7.89 E+00$ & $2.19 E+00$ & perm \\
\hline 163.6 & $1.05 E+01$ & $2.91 E+00$ & perm \\
\hline 160.6 & $5.54 E+01$ & $1.54 \mathrm{E}+01$ & core \\
\hline 159.6 & $3.49 E+01$ & $9.69 E+00$ & core \\
\hline 158.8 & $1.46 E+01$ & $4.06 E+00$ & perm \\
\hline 157.8 & $1.20 E+01$ & $3.33 E+00$ & perm \\
\hline 154.6 & $4.60 E+01$ & $1.28 \mathrm{E}+01$ & core \\
\hline 153.6 & $4.60 E+01$ & $1.28 \mathrm{E}+01$ & core \\
\hline 152.6 & $4.60 E+01$ & $1.28 \mathrm{E}+01$ & core \\
\hline 151.6 & $1.00 E+02$ & $2.78 E+01$ & $\mathrm{cpt}$ \\
\hline 150.6 & $1.00 E+02$ & $2.78 E+01$ & cpt \\
\hline 149.7 & $3.85 E+01$ & $1.07 E+01$ & perm \\
\hline 148.6 & $4.39 E+01$ & $1.22 E+01$ & perm \\
\hline
\end{tabular}


Conductivity Data at RBW2CL

\begin{tabular}{|c|c|c|c|}
\hline $\begin{array}{c}\text { Elevation } \\
\text { (ft) }\end{array}$ & $\begin{array}{c}\mathrm{Kh} \\
\text { (ft/day) }\end{array}$ & $\begin{array}{c}\mathrm{Kv} \\
\text { (ft/day) }\end{array}$ & $\begin{array}{c}\text { Data } \\
\text { Source }\end{array}$ \\
\hline 146.6 & $3.89 E+01$ & $1.08 \mathrm{E}+01$ & cpt \\
\hline 145.6 & $3.51 \mathrm{E}+01$ & $9.76 \mathrm{E}+00$ & $\mathrm{cpt}$ \\
\hline 144.6 & $3.49 E+01$ & $9.69 \mathrm{E}+00$ & core \\
\hline 143.1 & $3.41 E+01$ & $9.47 \mathrm{E}+00$ & perm \\
\hline 141.6 & $1.10 E+00$ & $3.04 \mathrm{E}-01$ & $\mathrm{cpt}$ \\
\hline 140.6 & $3.84 E+01$ & $1.07 E+01$ & cpt \\
\hline 139.8 & $4.84 \mathrm{E}+01$ & $1.34 E+01$ & perm \\
\hline 139.1 & $3.79 E+01$ & $1.05 E+01$ & perm \\
\hline 138.6 & $5.05 E+01$ & $1.40 \mathrm{E}+01$ & core \\
\hline 137.5 & $2.72 E+01$ & $7.56 \mathrm{E}+00$ & perm \\
\hline 137.1 & $5.12 E+01$ & $1.42 \mathrm{E}+01$ & perm \\
\hline 136.6 & $3.49 E+01$ & $9.69 \mathrm{E}+00$ & core \\
\hline 135.1 & $2.46 \mathrm{E}+01$ & $6.83 E+00$ & perm \\
\hline 134.1 & $3.87 E-01$ & $1.08 \mathrm{E}-01$ & perm \\
\hline 133.6 & $4.27 E+01$ & $1.19 \mathrm{E}+01$ & core \\
\hline 132.3 & $1.89 E+00$ & $5.25 \mathrm{E}-01$ & perm \\
\hline 131.6 & $6.76 \mathrm{E}-01$ & $1.88 \mathrm{E}-01$ & perm \\
\hline 130.6 & $2.77 \mathrm{E}+01$ & $7.68 \mathrm{E}+00$ & core \\
\hline 129.7 & $1.74 \mathrm{E}+00$ & $4.84 \mathrm{E}-01$ & perm \\
\hline 128.6 & $3.62 \mathrm{E}+01$ & $1.01 \mathrm{E}+01$ & core \\
\hline 127.8 & $1.75 \mathrm{E}+00$ & $4.87 \mathrm{E}-01$ & perm \\
\hline 127.1 & $1.61 E+00$ & $4.47 \mathrm{E}-01$ & perm \\
\hline 126.6 & $1.28 \mathrm{E}+01$ & $3.56 \mathrm{E}+00$ & core \\
\hline 125.1 & $2.31 \mathrm{E}+00$ & $6.41 \mathrm{E}-01$ & perm \\
\hline 124.1 & $4.42 \mathrm{E}+00$ & $1.23 E+00$ & perm \\
\hline 123.6 & $2.41 E+01$ & $6.69 \mathrm{E}+00$ & core \\
\hline 122.8 & $3.26 E+00$ & $9.04 \mathrm{E}-01$ & perm \\
\hline 122.1 & $6.55 \mathrm{E}+00$ & $1.82 E+00$ & perm \\
\hline 121.1 & $1.09 E+01$ & $3.02 E+00$ & perm \\
\hline 120.1 & $6.16 \mathrm{E}+00$ & $1.71 \mathrm{E}+00$ & perm \\
\hline 119.6 & $2.64 E+01$ & $7.34 \mathrm{E}+00$ & core \\
\hline 118.9 & $1.20 \mathrm{E}+00$ & $3.34 \mathrm{E}-01$ & perm \\
\hline 118.1 & $1.39 E+00$ & 3.87E-01 & perm \\
\hline 117.1 & $1.97 E+00$ & $5.47 \mathrm{E}-01$ & perm \\
\hline 116.6 & $3.62 E+01$ & $1.01 \mathrm{E}+01$ & core \\
\hline 115.8 & $1.29 E+00$ & $3.59 \mathrm{E}-01$ & perm \\
\hline 114.9 & $4.08 E+00$ & $1.13 E+00$ & perm \\
\hline 114.1 & $2.11 E+00$ & $5.86 \mathrm{E}-01$ & perm \\
\hline 113.1 & $1.27 E+00$ & $3.51 \mathrm{E}-01$ & perm \\
\hline 112.6 & $7.86 \mathrm{E}+00$ & $2.18 E+00$ & core \\
\hline 111.8 & $3.50 E+00$ & $9.72 \mathrm{E}-01$ & perm \\
\hline 111.1 & $2.24 E+00$ & $6.22 \mathrm{E}-01$ & perm \\
\hline 110.1 & 7.97E-01 & $2.21 \mathrm{E}-01$ & perm \\
\hline 109.1 & $3.84 \mathrm{E}-01$ & $1.07 \mathrm{E}-01$ & perm \\
\hline 108.6 & $1.25 E+01$ & $3.47 \mathrm{E}+00$ & core \\
\hline 104.5 & $1.01 E+01$ & $2.81 E+00$ & perm \\
\hline 103.8 & $1.14 \mathrm{E}+01$ & $3.17 \mathrm{E}+00$ & perm \\
\hline 102.8 & $1.58 \mathrm{E}-01$ & $4.39 \mathrm{E}-02$ & perm \\
\hline
\end{tabular}


Conductivity Data at RBW2CL

\begin{tabular}{|c|c|c|c|}
\hline $\begin{array}{c}\text { Elevation } \\
\text { (ft) }\end{array}$ & $\begin{array}{c}\text { Kh } \\
\text { (ft/day) }\end{array}$ & $\begin{array}{c}\text { Kv } \\
\text { (ft/day) }\end{array}$ & $\begin{array}{c}\text { Data } \\
\text { Source }\end{array}$ \\
\hline 100.1 & $4.78 \mathrm{E}+00$ & $1.33 \mathrm{E}+00$ & perm \\
\hline 99.1 & $1.08 \mathrm{E}+01$ & $3.00 \mathrm{E}+00$ & perm \\
\hline 98.6 & $3.83 \mathrm{E}+01$ & $1.06 \mathrm{E}+01$ & core \\
\hline 97.8 & $1.27 \mathrm{E}+01$ & $3.53 \mathrm{E}+00$ & perm \\
\hline 96.7 & $1.02 \mathrm{E}+01$ & $2.83 \mathrm{E}+00$ & perm \\
\hline 95.1 & $6.86 \mathrm{E}+00$ & $1.91 \mathrm{E}+00$ & perm \\
\hline 94.6 & $1.82 \mathrm{E}+01$ & $5.07 \mathrm{E}+00$ & core \\
\hline 93.9 & $1.91 \mathrm{E}+00$ & $5.31 \mathrm{E}-01$ & perm \\
\hline 93.1 & $3.55 \mathrm{E}+00$ & $9.87 \mathrm{E}-01$ & perm \\
\hline 92.6 & $2.90 \mathrm{E}+01$ & $8.05 \mathrm{E}+00$ & core \\
\hline 91.6 & $1.80 \mathrm{E}+00$ & $5.01 \mathrm{E}-01$ & core \\
\hline 89.9 & $3.07 \mathrm{E}+00$ & $8.52 \mathrm{E}-01$ & perm \\
\hline 88.6 & $2.51 \mathrm{E}-05$ & $6.97 \mathrm{E}-06$ & core \\
\hline 87.6 & $2.40 \mathrm{E}-05$ & $6.66 \mathrm{E}-06$ & core \\
\hline 86.6 & $2.40 \mathrm{E}-05$ & $6.66 \mathrm{E}-06$ & core \\
\hline 85.6 & $2.57 \mathrm{E}-05$ & $7.14 \mathrm{E}-06$ & core \\
\hline 84.6 & $1.80 \mathrm{E}+00$ & $5.01 \mathrm{E}-01$ & core \\
\hline 83.6 & $4.55 \mathrm{E}+00$ & $1.26 \mathrm{E}+00$ & core \\
\hline 82.8 & $6.53 \mathrm{E}+01$ & $1.81 \mathrm{E}+01$ & perm \\
\hline 79.1 & $5.34 \mathrm{E}+01$ & $1.48 \mathrm{E}+01$ & perm \\
\hline 78.1 & $4.76 \mathrm{E}+01$ & $1.32 \mathrm{E}+01$ & perm \\
\hline 77.1 & $5.32 \mathrm{E}+01$ & $1.48 \mathrm{E}+01$ & perm \\
\hline 76.6 & $7.24 \mathrm{E}+01$ & $2.01 \mathrm{E}+01$ & core \\
\hline & & & \\
\hline
\end{tabular}


Conductivity Data at RBW2CU

\begin{tabular}{|c|c|c|c|}
\hline $\begin{array}{c}\text { Elevation } \\
\text { (ft) }\end{array}$ & $\begin{array}{c}\text { Kh } \\
\text { (ft/day) }\end{array}$ & $\begin{array}{c}\mathrm{Kv} \\
\text { (ft/day) }\end{array}$ & $\begin{array}{l}\text { Data } \\
\text { Source }\end{array}$ \\
\hline 204.9 & $7.74 E+00$ & $3.87 \mathrm{E}-02$ & perm \\
\hline 204.2 & $1.52 E+01$ & $7.58 \mathrm{E}-02$ & perm \\
\hline 203.2 & $1.12 \mathrm{E}+01$ & $5.58 \mathrm{E}-02$ & perm \\
\hline 202.7 & $5.05 E+01$ & $1.40 E+01$ & core \\
\hline 199.8 & $1.37 E+01$ & 6.82E-02 & perm \\
\hline 199.2 & $1.47 E+01$ & $7.35 \mathrm{E}-02$ & perm \\
\hline 198.7 & $4.60 \mathrm{E}+01$ & $1.28 \mathrm{E}+01$ & core \\
\hline 197.8 & $2.06 E+01$ & $1.03 E-01$ & perm \\
\hline 196.9 & $2.60 E+01$ & 1.30E-01 & perm \\
\hline 196.2 & $4.03 E+01$ & $2.02 \mathrm{E}-01$ & perm \\
\hline 194.8 & $2.60 E+01$ & 1.30E-01 & perm \\
\hline 194.2 & $3.06 E+01$ & $1.53 \mathrm{E}-01$ & perm \\
\hline 193.7 & $4.19 E+01$ & $1.16 E+01$ & core \\
\hline 190.2 & $3.98 E+01$ & $1.99 \mathrm{E}-01$ & perm \\
\hline 189.7 & $5.54 \mathrm{E}+01$ & $1.54 \mathrm{E}+01$ & core \\
\hline 188.7 & $5.28 \mathrm{E}-04$ & 1.47E-04 & core \\
\hline 185.0 & $1.10 E+01$ & $5.50 \mathrm{E}-02$ & perm \\
\hline 184.2 & $2.79 E+01$ & $1.39 \mathrm{E}-01$ & perm \\
\hline 183.2 & $3.32 E+01$ & $1.66 \mathrm{E}-01$ & perm \\
\hline 182.7 & $1.39 \mathrm{E}-01$ & $3.86 E-02$ & core \\
\hline 180.0 & $3.67 E+01$ & $1.84 \mathrm{E}-01$ & perm \\
\hline 177.7 & 4.41E-05 & 1.23E-05 & core \\
\hline 176.8 & $8.91 E+00$ & 4.46E-02 & perm \\
\hline 175.2 & $1.38 E+01$ & $6.91 \mathrm{E}-02$ & perm \\
\hline 174.7 & $2.54 \mathrm{E}-03$ & $7.05 E-04$ & core \\
\hline 173.8 & $1.15 E+01$ & $5.73 E-02$ & perm \\
\hline 173.2 & $4.33 E+01$ & $2.17 E-01$ & perm \\
\hline 172.7 & 8.91E-04 & $2.48 E-04$ & core \\
\hline 171.7 & $8.36 E-05$ & $2.32 E-05$ & core \\
\hline 170.7 & $8.36 E-05$ & $2.32 \mathrm{E}-05$ & core \\
\hline 169.7 & 4.41E-05 & 1.23E-05 & core \\
\hline 168.7 & $3.20 E-05$ & 8.90E-06 & core \\
\hline 167.4 & $7.08 E+00$ & $1.97 E+00$ & perm \\
\hline 166.8 & $2.47 E+01$ & $6.85 E+00$ & perm \\
\hline 166.2 & $4.80 E+01$ & $1.33 E+01$ & perm \\
\hline 165.7 & $4.30 E+00$ & $1.20 E+00$ & core \\
\hline 164.8 & $1.16 E+01$ & $3.21 E+00$ & perm \\
\hline 164.2 & $5.62 E+00$ & $1.56 E+00$ & perm \\
\hline 162.7 & $1.05 E+01$ & $2.91 E+00$ & perm \\
\hline 162.2 & $2.66 \mathrm{E}+01$ & $7.40 E+00$ & perm \\
\hline 161.2 & $3.32 E+01$ & $9.21 E+00$ & perm \\
\hline 160.7 & $5.05 E+01$ & $1.40 \mathrm{E}+01$ & core \\
\hline 159.7 & $2.34 \mathrm{E}+00$ & $6.49 \mathrm{E}-01$ & core \\
\hline 158.5 & $2.69 E+01$ & $7.46 E+00$ & perm \\
\hline 157.9 & $2.31 E+01$ & $6.40 E+00$ & perm \\
\hline 157.2 & $1.62 E+01$ & $4.50 E+00$ & perm \\
\hline 156.2 & $2.97 E+01$ & $8.25 E+00$ & perm \\
\hline 155.2 & $5.27 E+01$ & $1.47 E+01$ & perm \\
\hline
\end{tabular}


Conductivity Data at RBW2CU

\begin{tabular}{|c|c|c|c|}
\hline $\begin{array}{c}\text { Elevation } \\
\text { (ft) }\end{array}$ & $\begin{array}{c}\text { Kh } \\
\text { (ft/day) }\end{array}$ & $\begin{array}{c}\text { Kv } \\
\text { (ft/day) }\end{array}$ & $\begin{array}{c}\text { Data } \\
\text { Source }\end{array}$ \\
\hline 154.7 & $3.18 \mathrm{E}+01$ & $8.83 \mathrm{E}+00$ & core \\
\hline 153.7 & $3.49 \mathrm{E}+01$ & $9.69 \mathrm{E}+00$ & core \\
\hline 152.7 & $3.49 \mathrm{E}+01$ & $9.69 \mathrm{E}+00$ & core \\
\hline 149.8 & $3.44 \mathrm{E}+01$ & $9.55 \mathrm{E}+00$ & perm \\
\hline 147.7 & $4.60 \mathrm{E}+01$ & $1.28 \mathrm{E}+01$ & core \\
\hline 144.7 & $4.60 \mathrm{E}+01$ & $1.28 \mathrm{E}+01$ & core \\
\hline 143.7 & $5.54 \mathrm{E}+01$ & $1.54 \mathrm{E}+01$ & core \\
\hline 142.9 & $3.22 \mathrm{E}+01$ & $8.95 \mathrm{E}+00$ & perm \\
\hline
\end{tabular}


Conductivity Data at $\mathrm{RCH} 1$

\begin{tabular}{|c|c|c|c|}
\hline $\begin{array}{c}\text { Elevation } \\
\text { (ft) }\end{array}$ & $\begin{array}{c}\text { Kh } \\
\text { (ft/day) }\end{array}$ & $\begin{array}{c}\mathrm{Kv} \\
\text { (ft/day) }\end{array}$ & $\begin{array}{l}\text { Data } \\
\text { Source }\end{array}$ \\
\hline 302.1 & $3.73 E+00$ & $1.04 E+00$ & cpt \\
\hline 301.1 & $5.99 \mathrm{E}+00$ & $1.66 \mathrm{E}+00$ & cpt \\
\hline 300.1 & $3.70 \mathrm{E}-04$ & $1.00 \mathrm{E}-04$ & cpt \\
\hline 299.1 & $5.43 \mathrm{E}+00$ & $1.51 \mathrm{E}+00$ & cpt \\
\hline 298.1 & $4.16 \mathrm{E}-03$ & $1.15 E-03$ & $\mathrm{cpt}$ \\
\hline 296.6 & $1.01 \mathrm{E}-02$ & $2.81 \mathrm{E}-03$ & perm \\
\hline 295.1 & $2.33 E-03$ & $6.50 \mathrm{E}-04$ & $\mathrm{cpt}$ \\
\hline 294.1 & $3.45 E-03$ & $9.60 \mathrm{E}-04$ & $\mathrm{cpt}$ \\
\hline 293.1 & $2.38 \mathrm{E}-03$ & $6.60 E-04$ & $\mathrm{cpt}$ \\
\hline 291.6 & $9.35 \mathrm{E}-03$ & $3.97 E-03$ & perm \\
\hline 290.4 & $6.03 \mathrm{E}+00$ & $6.03 E-02$ & perm \\
\hline 289.1 & $2.87 \mathrm{E}+00$ & $7.97 E-01$ & core \\
\hline 288.1 & $3.36 \mathrm{E}-03$ & $9.30 \mathrm{E}-04$ & cpt \\
\hline 287.1 & $2.84 \mathrm{E}-01$ & $7.89 \mathrm{E}-02$ & core \\
\hline 286.1 & $2.84 E-01$ & $7.89 \mathrm{E}-02$ & core \\
\hline 285.3 & $3.59 \mathrm{E}+00$ & $3.59 \mathrm{E}-02$ & perm \\
\hline 284.1 & $4.56 \mathrm{E}+00$ & $1.27 \mathrm{E}+00$ & core \\
\hline 283.1 & 4.04E-01 & 1.12E-01 & cpt \\
\hline 281.6 & $4.79 E-03$ & $1.33 \mathrm{E}-03$ & perm \\
\hline 280.4 & $1.39 \mathrm{E}+01$ & 1.39E-01 & perm \\
\hline 279.6 & $8.59 \mathrm{E}+00$ & $8.59 E-02$ & perm \\
\hline 278.3 & $2.47 E+00$ & $2.47 \mathrm{E}-02$ & perm \\
\hline 276.6 & $4.50 \mathrm{E}-02$ & $1.25 \mathrm{E}-02$ & perm \\
\hline 275.6 & $1.11 \mathrm{E}+01$ & $1.11 \mathrm{E}-01$ & perm \\
\hline 274.6 & $1.35 \mathrm{E}+01$ & $1.35 \mathrm{E}-01$ & perm \\
\hline 273.6 & $1.53 \mathrm{E}+01$ & $1.53 \mathrm{E}-01$ & perm \\
\hline 272.3 & $9.29 \mathrm{E}+00$ & $9.29 E-02$ & perm \\
\hline 271.6 & $1.33 \mathrm{E}+00$ & $1.33 \mathrm{E}-02$ & perm \\
\hline 270.6 & $1.95 \mathrm{E}+01$ & $1.95 \mathrm{E}-01$ & perm \\
\hline 269.6 & $1.94 \mathrm{E}+01$ & $1.94 \mathrm{E}-01$ & perm \\
\hline 268.6 & $1.83 \mathrm{E}+01$ & $1.83 \mathrm{E}-01$ & perm \\
\hline 267.6 & $1.01 \mathrm{E}+01$ & $1.01 \mathrm{E}-01$ & perm \\
\hline 266.6 & $1.11 \mathrm{E}+01$ & 1.11E-01 & perm \\
\hline 265.6 & $1.00 \mathrm{E}+01$ & $1.00 \mathrm{E}-01$ & perm \\
\hline 264.3 & $6.46 E+00$ & $6.46 \mathrm{E}-02$ & perm \\
\hline 263.6 & $4.41 E+00$ & $4.41 \mathrm{E}-02$ & perm \\
\hline 262.4 & $7.90 \mathrm{E}-01$ & $7.90 \mathrm{E}-03$ & perm \\
\hline 261.3 & $1.00 E+00$ & $1.00 \mathrm{E}-02$ & perm \\
\hline 260.6 & $1.25 E+01$ & $1.25 \mathrm{E}-01$ & perm \\
\hline 259.1 & $1.15 E+01$ & $3.19 \mathrm{E}+00$ & core \\
\hline 257.6 & $1.75 \mathrm{E}+00$ & $1.75 \mathrm{E}-02$ & perm \\
\hline 256.3 & $1.52 E+01$ & $1.52 \mathrm{E}-01$ & perm \\
\hline 255.2 & $2.04 \mathrm{E}+01$ & $2.04 \mathrm{E}-01$ & perm \\
\hline 254.6 & $5.61 E+00$ & $5.61 \mathrm{E}-02$ & perm \\
\hline 253.1 & $1.92 E+01$ & $5.33 E+00$ & cpt \\
\hline 252.3 & $1.94 E+01$ & $1.94 \mathrm{E}-01$ & perm \\
\hline 251.6 & $2.69 E+00$ & $2.69 \mathrm{E}-02$ & perm \\
\hline 250.3 & $5.12 \mathrm{E}+00$ & $5.12 \mathrm{E}-02$ & perm \\
\hline
\end{tabular}


Conductivity Data at RCH1

\begin{tabular}{|c|c|c|c|}
\hline $\begin{array}{c}\text { Elevation } \\
\text { (ft) }\end{array}$ & $\begin{array}{c}\text { Kh } \\
\text { (ft/day) }\end{array}$ & $\begin{array}{c}\mathrm{Kv} \\
\text { (ft/day) }\end{array}$ & $\begin{array}{l}\text { Data } \\
\text { Source }\end{array}$ \\
\hline 249.1 & $1.08 E+01$ & $3.00 E+00$ & cpt \\
\hline 247.6 & $6.52 E-01$ & $4.54 E-02$ & perm \\
\hline 246.6 & $2.90 E+00$ & $2.90 \mathrm{E}-02$ & perm \\
\hline 245.4 & $9.80 \mathrm{E}-01$ & $4.90 \mathrm{E}-03$ & perm \\
\hline 244.6 & $1.23 E+01$ & $6.15 E-02$ & perm \\
\hline 243.4 & $1.12 E+01$ & $5.60 \mathrm{E}-02$ & perm \\
\hline 242.6 & $2.87 E+01$ & $1.44 \mathrm{E}-01$ & perm \\
\hline 241.6 & $4.11 E+01$ & $2.06 E-01$ & perm \\
\hline 240.3 & $2.67 E+01$ & $1.34 \mathrm{E}-01$ & perm \\
\hline 239.6 & $3.57 E+01$ & $1.78 \mathrm{E}-01$ & perm \\
\hline 238.1 & $3.98 E+01$ & $1.10 E+01$ & cpt \\
\hline 237.4 & $2.90 E+01$ & $1.45 E-01$ & perm \\
\hline 236.6 & $3.15 E+01$ & $1.58 \mathrm{E}-01$ & perm \\
\hline 235.6 & $2.46 E+01$ & $1.23 E-01$ & perm \\
\hline 234.6 & $1.77 E+01$ & 8.87E-02 & perm \\
\hline 233.6 & $1.54 E+01$ & $7.71 \mathrm{E}-02$ & perm \\
\hline 232.1 & $5.41 E+00$ & $2.71 \mathrm{E}-02$ & perm \\
\hline 231.2 & $1.07 E+01$ & $5.36 \mathrm{E}-02$ & perm \\
\hline 230.6 & $4.80 E+00$ & $2.40 \mathrm{E}-02$ & perm \\
\hline 229.3 & $1.91 E+01$ & $9.54 \mathrm{E}-02$ & perm \\
\hline 228.3 & $1.16 \mathrm{E}+01$ & $5.80 \mathrm{E}-02$ & perm \\
\hline 227.3 & $2.13 E+01$ & 1.07E-01 & perm \\
\hline 226.3 & $1.63 E+01$ & $8.16 \mathrm{E}-02$ & perm \\
\hline 225.5 & $2.23 E+01$ & 1.12E-01 & perm \\
\hline 224.1 & $1.39 E-01$ & $3.86 \mathrm{E}-02$ & core \\
\hline 222.6 & $4.50 E+01$ & $2.25 E-01$ & perm \\
\hline 221.6 & $2.70 E+01$ & 1.35E-01 & perm \\
\hline 220.6 & $2.62 E+01$ & $1.31 \mathrm{E}-01$ & perm \\
\hline 219.6 & $2.70 E+01$ & $1.35 \mathrm{E}-01$ & perm \\
\hline 217.6 & $3.09 E+01$ & $1.55 E-01$ & perm \\
\hline 216.6 & $3.07 E+01$ & $1.54 \mathrm{E}-01$ & perm \\
\hline 215.6 & $4.65 E+01$ & 2.33E-01 & perm \\
\hline 214.6 & $9.00 E+01$ & $4.50 \mathrm{E}-01$ & perm \\
\hline 213.6 & $9.15 E+01$ & $4.57 E-01$ & perm \\
\hline 211.6 & $1.22 E+02$ & $6.08 \mathrm{E}-01$ & perm \\
\hline 210.1 & $2.52 E+01$ & $6.99 E+00$ & $\mathrm{cpt}$ \\
\hline 207.6 & $1.11 E+02$ & $5.56 E-01$ & perm \\
\hline 206.6 & $1.00 E+02$ & $5.01 E-01$ & perm \\
\hline 205.6 & $4.90 E+01$ & $2.45 E-01$ & perm \\
\hline 202.1 & $5.28 E-04$ & 1.47E-04 & core \\
\hline 201.4 & $3.18 E+01$ & $1.59 \mathrm{E}-01$ & perm \\
\hline 200.6 & $2.80 E+01$ & $1.40 \mathrm{E}-01$ & perm \\
\hline 199.6 & $6.22 E+01$ & 3.11E-01 & perm \\
\hline 198.6 & $6.24 E+01$ & $3.12 \mathrm{E}-01$ & perm \\
\hline 197.6 & $5.18 E+01$ & $2.59 \mathrm{E}-01$ & perm \\
\hline 196.6 & $4.35 E+01$ & $2.18 \mathrm{E}-01$ & perm \\
\hline 195.6 & $6.40 E+01$ & $3.20 \mathrm{E}-01$ & perm \\
\hline 194.6 & $6.47 E+01$ & $3.24 E-01$ & perm \\
\hline
\end{tabular}


Conductivity Data at RCH1

\begin{tabular}{|c|c|c|c|}
\hline $\begin{array}{c}\text { Elevation } \\
\text { (ft) }\end{array}$ & $\begin{array}{c}\text { Kh } \\
\text { (ft/day) }\end{array}$ & $\begin{array}{c}\text { Kv } \\
\text { (ft/day) }\end{array}$ & $\begin{array}{c}\text { Data } \\
\text { Source }\end{array}$ \\
\hline 192.6 & $7.70 \mathrm{E}+01$ & $3.85 \mathrm{E}-01$ & perm \\
\hline 191.6 & $2.82 \mathrm{E}+01$ & $1.41 \mathrm{E}-01$ & perm \\
\hline 190.6 & $9.23 \mathrm{E}+01$ & $4.61 \mathrm{E}-01$ & perm \\
\hline 189.6 & $7.76 \mathrm{E}+01$ & $3.88 \mathrm{E}-01$ & perm \\
\hline 188.6 & $6.73 \mathrm{E}+01$ & $3.37 \mathrm{E}-01$ & perm \\
\hline 187.6 & $1.02 \mathrm{E}+02$ & $5.10 \mathrm{E}-01$ & perm \\
\hline 186.6 & $2.78 \mathrm{E}+01$ & $1.39 \mathrm{E}-01$ & perm \\
\hline 185.6 & $7.59 \mathrm{E}+01$ & $3.80 \mathrm{E}-01$ & perm \\
\hline 184.6 & $8.79 \mathrm{E}+01$ & $4.40 \mathrm{E}-01$ & perm \\
\hline 183.6 & $8.31 \mathrm{E}+01$ & $4.15 \mathrm{E}-01$ & perm \\
\hline 182.4 & $7.57 \mathrm{E}+01$ & $3.78 \mathrm{E}-01$ & perm \\
\hline 181.6 & $7.46 \mathrm{E}+01$ & $3.73 \mathrm{E}-01$ & perm \\
\hline 180.6 & $5.70 \mathrm{E}+01$ & $2.85 \mathrm{E}-01$ & perm \\
\hline 179.6 & $3.45 \mathrm{E}+01$ & $1.73 \mathrm{E}-01$ & perm \\
\hline 178.1 & $2.72 \mathrm{E}-05$ & $7.56 \mathrm{E}-06$ & core \\
\hline 176.6 & $1.53 \mathrm{E}-02$ & $4.25 \mathrm{E}-03$ & perm \\
\hline 175.0 & $2.72 \mathrm{E}+01$ & $7.57 \mathrm{E}+00$ & perm \\
\hline 174.4 & $5.55 \mathrm{E}+01$ & $1.54 \mathrm{E}+01$ & perm \\
\hline 173.0 & $3.62 \mathrm{E}+01$ & $1.01 \mathrm{E}+01$ & perm \\
\hline 172.6 & $9.21 \mathrm{E}+01$ & $2.56 \mathrm{E}+01$ & perm \\
\hline 171.3 & $6.45 \mathrm{E}+01$ & $1.79 \mathrm{E}+01$ & perm \\
\hline 170.6 & $9.96 \mathrm{E}+01$ & $2.77 \mathrm{E}+01$ & perm \\
\hline 169.6 & $1.12 \mathrm{E}+02$ & $3.12 \mathrm{E}+01$ & perm \\
\hline 168.6 & $7.38 \mathrm{E}+01$ & $2.05 \mathrm{E}+01$ & perm \\
\hline 167.6 & $9.22 \mathrm{E}+01$ & $2.56 \mathrm{E}+01$ & perm \\
\hline 166.6 & $1.13 \mathrm{E}+02$ & $3.15 \mathrm{E}+01$ & perm \\
\hline 164.6 & $8.46 \mathrm{E}+01$ & $2.35 \mathrm{E}+01$ & perm \\
\hline 162.6 & $6.30 \mathrm{E}+01$ & $1.75 \mathrm{E}+01$ & perm \\
\hline 161.6 & $1.07 \mathrm{E}+02$ & $2.98 \mathrm{E}+01$ & perm \\
\hline 160.6 & $9.43 \mathrm{E}+01$ & $2.62 \mathrm{E}+01$ & perm \\
\hline 159.6 & $1.35 \mathrm{E}+02$ & $3.76 \mathrm{E}+01$ & perm \\
\hline 157.6 & $4.82 \mathrm{E}+01$ & $1.34 \mathrm{E}+01$ & perm \\
\hline 156.6 & $5.15 \mathrm{E}+01$ & $1.43 \mathrm{E}+01$ & perm \\
\hline 155.6 & $8.43 \mathrm{E}+01$ & $2.34 \mathrm{E}+01$ & perm \\
\hline 154.0 & $5.38 \mathrm{E}+01$ & $1.49 \mathrm{E}+01$ & perm \\
\hline 153.6 & $5.00 \mathrm{E}+01$ & $1.39 \mathrm{E}+01$ & perm \\
\hline 152.2 & $5.54 \mathrm{E}+01$ & $1.54 \mathrm{E}+01$ & perm \\
\hline 151.1 & $6.08 \mathrm{E}+01$ & $1.69 \mathrm{E}+01$ & core \\
\hline 150.1 & $6.08 \mathrm{E}+01$ & $1.69 \mathrm{E}+01$ & core \\
\hline 149.1 & $6.08 \mathrm{E}+01$ & $1.69 \mathrm{E}+01$ & core \\
\hline 147.1 & $6.67 \mathrm{E}+01$ & $1.85 \mathrm{E}+01$ & core \\
\hline 146.1 & $6.67 \mathrm{E}+01$ & $1.85 \mathrm{E}+01$ & core \\
\hline 145.1 & $6.67 \mathrm{E}+01$ & $1.85 \mathrm{E}+01$ & core \\
\hline 144.1 & $6.67 \mathrm{E}+01$ & $1.85 \mathrm{E}+01$ & core \\
\hline 142.1 & $6.67 \mathrm{E}+01$ & $1.85 \mathrm{E}+01$ & core \\
\hline $6.67 \mathrm{E}+01$ & $1.85 \mathrm{E}+01$ & core \\
\hline 140.1 & $6.67 \mathrm{E}+01$ & $1.85 \mathrm{E}+01$ & core \\
\hline & $6.67 \mathrm{E}+01$ & $1.85 \mathrm{E}+01$ & core \\
\hline
\end{tabular}


Conductivity Data at RCH1

\begin{tabular}{|c|c|c|c|}
\hline $\begin{array}{c}\text { Elevation } \\
\text { (ft) }\end{array}$ & $\begin{array}{c}\text { Kh } \\
\text { (ft/day) }\end{array}$ & $\begin{array}{c}\mathrm{Kv} \\
\text { (ft/day) }\end{array}$ & $\begin{array}{c}\text { Data } \\
\text { Source }\end{array}$ \\
\hline$\overline{139.1}$ & $6.67 E+01$ & $1.85 E+01$ & core \\
\hline 138.1 & $6.67 E+01$ & $1.85 E+01$ & core \\
\hline 137.1 & $3.72 E+01$ & $1.03 E+01$ & core \\
\hline 136.1 & $3.72 E+01$ & $1.03 E+01$ & core \\
\hline 135.1 & $3.04 E+01$ & $8.43 E+00$ & core \\
\hline 134.1 & $3.97 \mathrm{E}+01$ & $1.10 E+01$ & core \\
\hline 133.1 & $3.76 E+01$ & $1.04 \mathrm{E}+01$ & core \\
\hline 132.1 & $4.27 E+01$ & $1.19 \mathrm{E}+01$ & core \\
\hline$\overline{131.1}$ & $5.14 \mathrm{E}+01$ & $1.43 E+01$ & core \\
\hline 130.1 & $5.14 \mathrm{E}+01$ & $1.43 E+01$ & core \\
\hline 127.1 & $3.06 E+01$ & $8.51 E+00$ & core \\
\hline 126.1 & $4.52 E+01$ & $1.26 \mathrm{E}+01$ & core \\
\hline 125.1 & $3.93 E+01$ & $1.09 \mathrm{E}+01$ & core \\
\hline 124.1 & $3.93 \mathrm{E}+01$ & $1.09 E+01$ & core \\
\hline 123.1 & $3.93 E+01$ & $1.09 E+01$ & core \\
\hline 122.1 & $5.63 E+00$ & $1.56 E+00$ & core \\
\hline 121.1 & $6.47 \mathrm{E}+00$ & $1.80 \mathrm{E}+00$ & core \\
\hline 120.1 & $3.38 \mathrm{E}+00$ & $9.40 \mathrm{E}-01$ & core \\
\hline 106.6 & $2.88 \mathrm{E}+00$ & 8.00E-01 & perm \\
\hline 105.1 & $3.96 \mathrm{E}+00$ & $1.10 \mathrm{E}+00$ & core \\
\hline 104.1 & $2.48 \mathrm{E}-04$ & $6.89 \mathrm{E}-05$ & core \\
\hline 103.1 & 5.34E-05 & $1.48 \mathrm{E}-05$ & core \\
\hline 102.1 & $1.15 E-04$ & $3.20 \mathrm{E}-05$ & core \\
\hline$\overline{101.1}$ & $2.71 E-05$ & $7.54 \mathrm{E}-06$ & core \\
\hline 100.1 & $2.56 \mathrm{E}-05$ & $7.11 \mathrm{E}-06$ & core \\
\hline 99.1 & $2.65 E-05$ & $7.35 \mathrm{E}-06$ & core \\
\hline 98.1 & $2.61 \mathrm{E}-05$ & $7.25 \mathrm{E}-06$ & core \\
\hline 97.1 & $2.69 \mathrm{E}-05$ & $7.46 \mathrm{E}-06$ & core \\
\hline 96.1 & 4.14E-05 & 1.15E-05 & core \\
\hline 95.1 & $3.76 \mathrm{E}-05$ & $1.04 \mathrm{E}-05$ & core \\
\hline 94.1 & 2.72E-05 & $7.56 \mathrm{E}-06$ & core \\
\hline 93.1 & $2.56 \mathrm{E}-05$ & $7.10 \mathrm{E}-06$ & core \\
\hline 92.1 & 2.54E-05 & $7.05 \mathrm{E}-06$ & core \\
\hline 91.1 & $1.15 E+01$ & $3.19 E+00$ & core \\
\hline 90.1 & $1.82 \mathrm{E}+01$ & $5.07 E+00$ & core \\
\hline 89.1 & $4.60 \mathrm{E}+01$ & $1.28 \mathrm{E}+01$ & core \\
\hline
\end{tabular}


Conductivity Data at RCP1A

\begin{tabular}{|c|c|c|c|}
\hline $\begin{array}{c}\text { Elevation } \\
\text { (ft) }\end{array}$ & $\begin{array}{c}\mathrm{Kh} \\
\text { (ft/day) }\end{array}$ & $\begin{array}{c}\mathrm{Kv} \\
\text { (ft/day) }\end{array}$ & $\begin{array}{c}\text { Data } \\
\text { Source }\end{array}$ \\
\hline 221.3 & $3.82 \mathrm{E}+01$ & $1.06 \mathrm{E}+01$ & core \\
\hline 220.3 & $2.89 \mathrm{E}+01$ & $8.04 \mathrm{E}+00$ & core \\
\hline 219.3 & $1.82 \mathrm{E}+01$ & $5.06 \mathrm{E}+00$ & core \\
\hline 218.3 & $2.89 \mathrm{E}+01$ & $8.04 \mathrm{E}+00$ & core \\
\hline 217.3 & $3.48 \mathrm{E}+01$ & $9.67 \mathrm{E}+00$ & core \\
\hline 216.3 & $2.89 \mathrm{E}+01$ & $8.04 \mathrm{E}+00$ & core \\
\hline 215.3 & $3.48 \mathrm{E}+01$ & $9.67 \mathrm{E}+00$ & core \\
\hline 214.3 & $4.19 \mathrm{E}+01$ & $1.16 \mathrm{E}+01$ & core \\
\hline 213.3 & $4.60 \mathrm{E}+01$ & $1.28 \mathrm{E}+01$ & core \\
\hline 212.3 & $3.82 \mathrm{E}+01$ & $1.06 \mathrm{E}+01$ & core \\
\hline 211.3 & $1.82 \mathrm{E}+01$ & $5.06 \mathrm{E}+00$ & core \\
\hline 210.3 & $1.15 \mathrm{E}-04$ & $3.20 \mathrm{E}-05$ & core \\
\hline 209.3 & $1.50 \mathrm{E}-03$ & $4.18 \mathrm{E}-04$ & core \\
\hline 208.3 & $2.89 \mathrm{E}+01$ & $8.04 \mathrm{E}+00$ & core \\
\hline 207.3 & $2.89 \mathrm{E}+01$ & $8.04 \mathrm{E}+00$ & core \\
\hline 206.3 & $4.19 \mathrm{E}+01$ & $1.16 \mathrm{E}+01$ & core \\
\hline 205.3 & $3.82 \mathrm{E}+01$ & $1.06 \mathrm{E}+01$ & core \\
\hline 204.3 & $5.05 \mathrm{E}+01$ & $1.40 \mathrm{E}+01$ & core \\
\hline 203.3 & $5.05 \mathrm{E}+01$ & $1.40 \mathrm{E}+01$ & core \\
\hline 202.3 & $4.60 \mathrm{E}+01$ & $1.28 \mathrm{E}+01$ & core \\
\hline 201.3 & $4.60 \mathrm{E}+01$ & $1.28 \mathrm{E}+01$ & core \\
\hline 200.3 & $3.82 \mathrm{E}+01$ & $1.06 \mathrm{E}+01$ & core \\
\hline 199.3 & $2.89 \mathrm{E}+01$ & $8.04 \mathrm{E}+00$ & core \\
\hline 198.3 & $2.89 \mathrm{E}+01$ & $8.04 \mathrm{E}+00$ & core \\
\hline 197.3 & $3.48 \mathrm{E}+01$ & $9.67 \mathrm{E}+00$ & core \\
\hline 196.3 & $3.48 \mathrm{E}+01$ & $9.67 \mathrm{E}+00$ & core \\
\hline 195.3 & $3.48 \mathrm{E}+01$ & $9.67 \mathrm{E}+00$ & core \\
\hline 194.3 & $3.82 \mathrm{E}+01$ & $1.06 \mathrm{E}+01$ & core \\
\hline 193.3 & $4.60 \mathrm{E}+01$ & $1.28 \mathrm{E}+01$ & core \\
\hline 192.3 & $4.60 \mathrm{E}+01$ & $1.28 \mathrm{E}+01$ & core \\
\hline 191.3 & $5.05 \mathrm{E}+01$ & $1.40 \mathrm{E}+01$ & core \\
\hline 190.3 & $4.19 \mathrm{E}+01$ & $1.16 \mathrm{E}+01$ & core \\
\hline 189.3 & $3.48 \mathrm{E}+01$ & $9.67 \mathrm{E}+00$ & core \\
\hline 188.3 & $2.89 \mathrm{E}+01$ & $8.04 \mathrm{E}+00$ & core \\
\hline 187.3 & $2.89 \mathrm{E}+01$ & $8.04 \mathrm{E}+00$ & core \\
\hline 186.3 & $3.48 \mathrm{E}+01$ & $9.67 \mathrm{E}+00$ & core \\
\hline 185.3 & $3.48 \mathrm{E}+01$ & $9.67 \mathrm{E}+00$ & core \\
\hline 184.3 & $5.05 \mathrm{E}+01$ & $1.40 \mathrm{E}+01$ & core \\
\hline 183.3 & $3.82 \mathrm{E}+01$ & $1.06 \mathrm{E}+01$ & core \\
\hline 182.3 & $2.89 \mathrm{E}+01$ & $8.04 \mathrm{E}+00$ & core \\
\hline 181.3 & $1.82 \mathrm{E}+01$ & $5.06 \mathrm{E}+00$ & core \\
\hline 180.3 & $1.82 \mathrm{E}+01$ & $5.06 \mathrm{E}+00$ & core \\
\hline 179.3 & $1.59 \mathrm{E}-04$ & $4.40 \mathrm{E}-05$ & core \\
\hline 178.3 & $4.41 \mathrm{E}-05$ & $1.23 \mathrm{E}-05$ & core \\
\hline 175.3 & $2.54 \mathrm{E}-03$ & $7.05 \mathrm{E}-04$ & core \\
\hline 172.3 & $1.39 \mathrm{E}-01$ & $3.86 \mathrm{E}-02$ & core \\
\hline & $1.39 \mathrm{E}-01$ & $3.86 \mathrm{E}-02$ & core \\
\hline
\end{tabular}


Conductivity Data at RCP1A

\begin{tabular}{|c|c|c|c|}
\hline $\begin{array}{l}\text { Elevation } \\
\text { (ft) }\end{array}$ & $\begin{array}{c}\text { Kh } \\
\text { (ft/day) }\end{array}$ & $\begin{array}{c}\mathbf{K v} \\
\text { (ft/day) }\end{array}$ & $\begin{array}{c}\text { Data } \\
\text { Source }\end{array}$ \\
\hline 171.3 & $1.82 E+01$ & $5.06 \mathrm{E}+00$ & core \\
\hline 170.3 & $2.89 \mathrm{E}+01$ & $8.04 E+00$ & core \\
\hline 169.3 & $2.89 E+01$ & $8.04 E+00$ & core \\
\hline 168.3 & $2.89 E+01$ & $8.04 E+00$ & core \\
\hline 167.3 & $1.82 \mathrm{E}+01$ & $5.06 \mathrm{E}+00$ & core \\
\hline 166.3 & 1.39E-01 & $3.86 \mathrm{E}-02$ & core \\
\hline 165.3 & $5.28 \mathrm{E}-04$ & 1.47E-04 & core \\
\hline 164.3 & $3.13 \mathrm{E}-04$ & $8.69 \mathrm{E}-05$ & core \\
\hline 163.3 & $1.82 E+01$ & $5.06 \mathrm{E}+00$ & core \\
\hline 162.3 & $2.54 \mathrm{E}-03$ & $7.05 \mathrm{E}-04$ & core \\
\hline 161.3 & $7.23 E+00$ & $2.01 E+00$ & core \\
\hline 160.3 & $7.23 E+00$ & $2.01 \mathrm{E}+00$ & core \\
\hline 159.3 & $1.82 E+01$ & $5.07 E+00$ & core \\
\hline 158.3 & $2.90 E+01$ & $8.05 E+00$ & core \\
\hline 157.3 & $4.60 \mathrm{E}+01$ & $1.28 \mathrm{E}+01$ & core \\
\hline 156.3 & $7.23 E+00$ & $2.01 E+00$ & core \\
\hline 155.3 & $7.23 E+00$ & $2.01 E+00$ & core \\
\hline 154.3 & $1.82 E+01$ & $5.07 E+00$ & core \\
\hline 153.3 & $1.82 E+01$ & $5.07 E+00$ & core \\
\hline 152.3 & $1.21 \mathrm{E}-03$ & 3.37E-04 & core \\
\hline 151.3 & $1.82 E+01$ & $5.07 E+00$ & core \\
\hline 150.3 & $2.86 \mathrm{E}+00$ & $7.96 \mathrm{E}-01$ & core \\
\hline 149.3 & $2.86 \mathrm{E}+00$ & $7.96 \mathrm{E}-01$ & core \\
\hline 148.3 & $4.55 E+00$ & $1.26 \mathrm{E}+00$ & core \\
\hline 147.3 & $1.82 E+01$ & $5.07 \mathrm{E}+00$ & core \\
\hline 146.3 & $1.82 E+01$ & $5.07 E+00$ & core \\
\hline 145.3 & $7.23 E+00$ & $2.01 E+00$ & core \\
\hline 144.3 & $7.23 E+00$ & $2.01 \mathrm{E}+00$ & core \\
\hline 143.3 & $1.15 E+01$ & $3.19 E+00$ & core \\
\hline 142.3 & $1.82 \mathrm{E}+01$ & $5.07 \mathrm{E}+00$ & core \\
\hline 141.3 & $4.55 E+00$ & $1.26 \mathrm{E}+00$ & core \\
\hline 140.3 & $7.23 E+00$ & $2.01 E+00$ & core \\
\hline 139.3 & $7.23 E+00$ & $2.01 E+00$ & core \\
\hline 138.3 & $1.82 E+01$ & $5.07 E+00$ & core \\
\hline 137.3 & $3.49 E+01$ & $9.69 E+00$ & core \\
\hline 136.3 & $2.90 E+01$ & $8.05 E+00$ & core \\
\hline 135.3 & $2.90 \mathrm{E}+01$ & $8.05 E+00$ & core \\
\hline 134.3 & $2.90 \mathrm{E}+01$ & $8.05 E+00$ & core \\
\hline 133.3 & $2.90 E+01$ & $8.05 E+00$ & core \\
\hline 132.3 & $2.90 E+01$ & $8.05 E+00$ & core \\
\hline 131.3 & $1.21 \mathrm{E}-03$ & $3.37 E-04$ & core \\
\hline 130.3 & $7.23 E+00$ & $2.01 E+00$ & core \\
\hline 129.3 & $7.23 E+00$ & $2.01 E+00$ & core \\
\hline 128.3 & $7.23 E+00$ & $2.01 E+00$ & core \\
\hline 127.3 & $7.23 E+00$ & $2.01 \mathrm{E}+00$ & core \\
\hline 126.3 & $2.90 E+01$ & $8.05 E+00$ & core \\
\hline 125.3 & $2.90 E+01$ & $8.05 E+00$ & core \\
\hline 124.3 & $1.15 E+01$ & $3.19 \mathrm{E}+00$ & core \\
\hline
\end{tabular}


Conductivity Data at RCP1A

\begin{tabular}{|c|c|c|c|}
\hline $\begin{array}{c}\text { Elevation } \\
\text { (ft) }\end{array}$ & $\begin{array}{c}\text { Kh } \\
\text { (ft/day) }\end{array}$ & $\begin{array}{c}\mathrm{Kv} \\
\text { (ft/day) }\end{array}$ & $\begin{array}{c}\text { Data } \\
\text { Source }\end{array}$ \\
\hline 123.3 & $2.90 \mathrm{E}+01$ & $8.05 \mathrm{E}+00$ & core \\
\hline 122.3 & $1.82 \mathrm{E}+01$ & $5.07 \mathrm{E}+00$ & core \\
\hline 121.3 & $1.82 \mathrm{E}+01$ & $5.07 \mathrm{E}+00$ & core \\
\hline 120.3 & $4.20 \mathrm{E}+01$ & $1.17 \mathrm{E}+01$ & core \\
\hline 119.3 & $5.05 \mathrm{E}+01$ & $1.40 \mathrm{E}+01$ & core \\
\hline 118.3 & $5.54 \mathrm{E}+01$ & $1.54 \mathrm{E}+01$ & core \\
\hline 117.3 & $3.49 \mathrm{E}+01$ & $9.69 \mathrm{E}+00$ & core \\
\hline 116.3 & $5.54 \mathrm{E}+01$ & $1.54 \mathrm{E}+01$ & core \\
\hline 115.3 & $2.07 \mathrm{E}-04$ & $5.76 \mathrm{E}-05$ & core \\
\hline 114.3 & $5.45 \mathrm{E}-05$ & $1.51 \mathrm{E}-05$ & core \\
\hline 113.3 & $6.90 \mathrm{E}-05$ & $1.92 \mathrm{E}-05$ & core \\
\hline 112.3 & $1.18 \mathrm{E}-04$ & $3.28 \mathrm{E}-05$ & core \\
\hline 111.3 & $1.24 \mathrm{E}-04$ & $3.45 \mathrm{E}-05$ & core \\
\hline 102.3 & $6.11 \mathrm{E}-05$ & $1.70 \mathrm{E}-05$ & core \\
\hline 101.3 & $8.69 \mathrm{E}-05$ & $2.41 \mathrm{E}-05$ & core \\
\hline 100.3 & $5.18 \mathrm{E}-05$ & $1.44 \mathrm{E}-05$ & core \\
\hline 99.3 & $5.18 \mathrm{E}-05$ & $1.44 \mathrm{E}-05$ & core \\
\hline 98.3 & $8.23 \mathrm{E}+00$ & $2.29 \mathrm{E}+00$ & core \\
\hline 97.3 & $4.91 \mathrm{E}+01$ & $1.36 \mathrm{E}+01$ & core \\
\hline 96.3 & $1.26 \mathrm{E}-04$ & $3.50 \mathrm{E}-05$ & core \\
\hline 95.3 & $3.29 \mathrm{E}+00$ & $9.14 \mathrm{E}-01$ & core \\
\hline 94.3 & $4.47 \mathrm{E}-05$ & $1.24 \mathrm{E}-05$ & core \\
\hline 93.3 & $5.96 \mathrm{E}-05$ & $1.66 \mathrm{E}-05$ & core \\
\hline 92.3 & $9.62 \mathrm{E}-05$ & $2.67 \mathrm{E}-05$ & core \\
\hline 91.3 & $8.80 \mathrm{E}-05$ & $2.44 \mathrm{E}-05$ & core \\
\hline 90.3 & $2.56 \mathrm{E}+00$ & $7.12 \mathrm{E}-01$ & core \\
\hline 82.3 & $1.88 \mathrm{E}-04$ & $5.23 \mathrm{E}-05$ & core \\
\hline 72.3 & $8.36 \mathrm{E}-05$ & $2.32 \mathrm{E}-05$ & core \\
\hline 71.3 & $1.15 \mathrm{E}+01$ & $3.19 \mathrm{E}+00$ & core \\
\hline 70.3 & $1.15 \mathrm{E}+01$ & $3.19 \mathrm{E}+00$ & core \\
\hline 69.3 & $1.82 \mathrm{E}+01$ & $5.07 \mathrm{E}+00$ & core \\
\hline 68.3 & $1.82 \mathrm{E}+01$ & $5.07 \mathrm{E}+00$ & core \\
\hline 67.3 & $1.15 \mathrm{E}+01$ & $3.19 \mathrm{E}+00$ & core \\
\hline 66.3 & $1.82 \mathrm{E}+01$ & $5.07 \mathrm{E}+00$ & core \\
\hline 65.3 & $2.90 \mathrm{E}+01$ & $8.05 \mathrm{E}+00$ & core \\
\hline 64.3 & $7.23 \mathrm{E}+00$ & $2.01 \mathrm{E}+00$ & core \\
\hline 63.3 & $2.90 \mathrm{E}+01$ & $8.05 \mathrm{E}+00$ & core \\
\hline 62.3 & $7.23 \mathrm{E}+00$ & $2.01 \mathrm{E}+00$ & core \\
\hline 61.3 & $3.49 \mathrm{E}+01$ & $9.69 \mathrm{E}+00$ & core \\
\hline 60.3 & $4.60 \mathrm{E}+01$ & $1.28 \mathrm{E}+01$ & core \\
\hline 59.3 & $4.20 \mathrm{E}+01$ & $1.17 \mathrm{E}+01$ & core \\
\hline 58.3 & $3.49 \mathrm{E}+01$ & $9.69 \mathrm{E}+00$ & core \\
\hline 57.3 & $2.90 \mathrm{E}+01$ & $8.05 \mathrm{E}+00$ & core \\
\hline 56.3 & $4.20 \mathrm{E}+01$ & $1.17 \mathrm{E}+01$ & core \\
\hline 54.3 & $3.49 \mathrm{E}+01$ & $9.69 \mathrm{E}+00$ & core \\
\hline 53.3 & $3.49 \mathrm{E}+01$ & $9.69 \mathrm{E}+00$ & core \\
\hline 52.3 & $3.49 \mathrm{E}+01$ & $9.69 \mathrm{E}+00$ & core \\
\hline $3.83 \mathrm{E}+01$ & $1.06 \mathrm{E}+01$ & core \\
\hline & & & \\
\hline 1.3 & & \\
\hline
\end{tabular}


Conductivity Data at RCP1A

\begin{tabular}{|c|c|c|c|}
\hline $\begin{array}{c}\text { Elevation } \\
\text { (ft) }\end{array}$ & $\begin{array}{c}\mathbf{K h} \\
\text { (ft/day) }\end{array}$ & $\begin{array}{c}\text { Kv } \\
\text { (ft/day) }\end{array}$ & $\begin{array}{c}\text { Data } \\
\text { Source }\end{array}$ \\
\hline 51.3 & $4.60 \mathrm{E}+01$ & $1.28 \mathrm{E}+01$ & core \\
\hline 50.3 & $4.60 \mathrm{E}+01$ & $1.28 \mathrm{E}+01$ & core \\
\hline 49.3 & $4.60 \mathrm{E}+01$ & $1.28 \mathrm{E}+01$ & core \\
\hline 48.3 & $4.60 \mathrm{E}+01$ & $1.28 \mathrm{E}+01$ & core \\
\hline 47.3 & $4.60 \mathrm{E}+01$ & $1.28 \mathrm{E}+01$ & core \\
\hline
\end{tabular}


Conductivity Data at RPC1CL

\begin{tabular}{|c|c|c|c|}
\hline $\begin{array}{l}\text { Elevation } \\
\text { (ft) }\end{array}$ & $\begin{array}{c}\mathrm{Kh} \\
\text { (ft/day) }\end{array}$ & $\begin{array}{c}\mathrm{Kv} \\
\text { (ft/day) }\end{array}$ & $\begin{array}{c}\text { Data } \\
\text { Source }\end{array}$ \\
\hline 303.8 & $3.51 \mathrm{E}+00$ & $9.74 \mathrm{E}-01$ & cpt \\
\hline 302.8 & $4.03 E+00$ & $1.12 \mathrm{E}+00$ & cpt \\
\hline 301.8 & $4.80 E+00$ & $1.33 E+00$ & $\mathrm{cpt}$ \\
\hline 300.8 & $4.79 E+00$ & $1.33 E+00$ & $\mathrm{cpt}$ \\
\hline 299.8 & $4.16 \mathrm{E}+00$ & $1.15 E+00$ & $\mathrm{cpt}$ \\
\hline 298.8 & $4.78 \mathrm{E}+00$ & $1.33 E+00$ & $\mathrm{cpt}$ \\
\hline 297.8 & $5.00 \mathrm{E}+00$ & $1.39 E+00$ & $\mathrm{cpt}$ \\
\hline 296.8 & $5.71 E+00$ & $1.59 E+00$ & $\mathrm{cpt}$ \\
\hline 295.8 & $8.63 E+00$ & $2.40 E+00$ & cpt \\
\hline 294.8 & $5.08 \mathrm{E}-01$ & $1.41 E-01$ & cpt \\
\hline 293.8 & $7.24 E+00$ & $2.01 E+00$ & core \\
\hline 292.3 & $1.55 E+00$ & $4.31 E-01$ & perm \\
\hline 290.8 & $7.24 E+00$ & $2.01 E+00$ & core \\
\hline 289.9 & $3.27 E+00$ & $9.08 \mathrm{E}-01$ & perm \\
\hline 288.8 & $1.15 E+01$ & $3.19 E+00$ & core \\
\hline 287.6 & $7.40 \mathrm{E}-01$ & $2.06 E-01$ & perm \\
\hline 286.8 & $7.24 \mathrm{E}+00$ & $2.01 E+00$ & core \\
\hline 285.7 & $6.10 \mathrm{E}-01$ & 1.69E-01 & perm \\
\hline 284.3 & $3.40 E+00$ & $3.40 \mathrm{E}-02$ & perm \\
\hline 283.1 & $3.66 E+00$ & $3.66 \mathrm{E}-02$ & perm \\
\hline 282 & $1.39 E+00$ & $1.39 E-02$ & perm \\
\hline 280.5 & $1.03 E+00$ & 1.03E-02 & perm \\
\hline 279.8 & $1.81 E+00$ & $5.02 E-01$ & core \\
\hline 278.8 & $1.14 E+00$ & $3.16 \mathrm{E}-01$ & core \\
\hline 277.8 & $1.14 \mathrm{E}+00$ & $3.16 E-01$ & core \\
\hline 276.8 & $1.14 E+00$ & $3.16 \mathrm{E}-01$ & core \\
\hline 275.8 & $1.14 \mathrm{E}+00$ & 3.16E-01 & core \\
\hline 274.3 & $1.12 \mathrm{E}-02$ & $3.12 E-03$ & perm \\
\hline 272.8 & $1.14 E+00$ & $3.16 \mathrm{E}-01$ & core \\
\hline 271.8 & $1.81 \mathrm{E}+00$ & $5.02 E-01$ & core \\
\hline 270.8 & $4.51 \mathrm{E}-01$ & $1.25 E-01$ & core \\
\hline 269.8 & $7.10 \mathrm{E}-04$ & $2.00 \mathrm{E}-04$ & $\mathrm{cpt}$ \\
\hline 268.8 & $1.14 E+00$ & $3.16 \mathrm{E}-01$ & core \\
\hline 267.8 & $1.81 \mathrm{E}+00$ & $5.02 E-01$ & core \\
\hline 266.8 & $2.87 E+00$ & 7.97E-01 & core \\
\hline 265.8 & $6.30 \mathrm{E}-04$ & 1.70E-04 & cpt \\
\hline 264.8 & $6.00 E-04$ & $1.70 E-04$ & cpt \\
\hline 264 & $8.60 \mathrm{E}-01$ & $8.60 \mathrm{E}-03$ & perm \\
\hline 262.9 & $2.55 \mathrm{E}+00$ & $2.55 E-02$ & perm \\
\hline 261.8 & $6.20 E-04$ & $1.70 E-04$ & $\mathrm{cpt}$ \\
\hline 260.8 & $5.36 \mathrm{E}+00$ & $1.49 E+00$ & cpt \\
\hline 259.3 & $2.50 \mathrm{E}+00$ & $2.50 E-02$ & perm \\
\hline 258.1 & $3.73 E+00$ & $3.73 E-02$ & perm \\
\hline 256.8 & $3.75 \mathrm{E}+00$ & $3.75 \mathrm{E}-02$ & perm \\
\hline 256.3 & $2.65 \mathrm{E}+00$ & $2.65 E-02$ & perm \\
\hline 254.8 & $5.54 \mathrm{E}+00$ & $1.54 E+00$ & $\mathrm{cpt}$ \\
\hline 254 & $3.88 \mathrm{E}+00$ & $3.88 E-02$ & perm \\
\hline 252.8 & $1.69 \mathrm{E}+00$ & 1.69E-02 & perm \\
\hline
\end{tabular}


Conductivity Data at RPC1CL

\begin{tabular}{|c|c|c|c|}
\hline $\begin{array}{c}\text { Elevation } \\
\text { (ft) }\end{array}$ & $\begin{array}{c}\text { Kh } \\
\text { (ft/day) }\end{array}$ & $\begin{array}{c}\mathrm{Kv} \\
\text { (ft/day) }\end{array}$ & $\begin{array}{c}\text { Data } \\
\text { Source }\end{array}$ \\
\hline 251.8 & $1.81 E+00$ & $5.02 E-01$ & core \\
\hline 250.8 & $5.28 E+00$ & $1.47 E+00$ & $\mathrm{cpt}$ \\
\hline 249.8 & $8.90 \mathrm{E}-04$ & $2.50 \mathrm{E}-04$ & $\mathrm{cpt}$ \\
\hline 248.8 & $4.56 \mathrm{E}+00$ & $1.27 E+00$ & core \\
\hline 248 & $1.60 \mathrm{E}+00$ & 1.60E-02 & perm \\
\hline 247.3 & $1.65 \mathrm{E}+00$ & 1.65E-02 & perm \\
\hline 246.3 & $1.84 E+00$ & $1.84 \mathrm{E}-02$ & perm \\
\hline 245.3 & $6.70 E-01$ & 6.70E-03 & perm \\
\hline 243.9 & $7.20 \mathrm{E}-01$ & $7.20 \mathrm{E}-03$ & perm \\
\hline 242.8 & $1.81 E+00$ & $5.02 E-01$ & core \\
\hline 241.8 & $1.81 E+00$ & $5.02 E-01$ & core \\
\hline 240.8 & $1.15 E+01$ & $3.19 E+00$ & core \\
\hline 239.3 & $4.40 E+00$ & $4.40 \mathrm{E}-02$ & perm \\
\hline 238.3 & $2.79 E+00$ & $2.79 \mathrm{E}-02$ & perm \\
\hline 237.3 & $1.58 \mathrm{E}+00$ & $1.58 \mathrm{E}-02$ & perm \\
\hline 236.3 & $2.80 E+00$ & $2.80 E-02$ & perm \\
\hline 234.9 & $3.73 E+00$ & $3.73 E-02$ & perm \\
\hline 234.2 & $3.84 \mathrm{E}+00$ & $3.84 \mathrm{E}-02$ & perm \\
\hline 233.3 & $2.87 E+00$ & $2.87 E-02$ & perm \\
\hline 232.3 & $2.21 E+00$ & $2.21 E-02$ & perm \\
\hline 230.3 & $2.01 E+00$ & 1.01E-02 & perm \\
\hline 228.3 & $7.96 \mathrm{E}-03$ & $2.21 E-03$ & perm \\
\hline 227.3 & $1.43 E-02$ & $3.97 \mathrm{E}-03$ & perm \\
\hline 227.3 & $2.65 E+00$ & $1.33 \mathrm{E}-02$ & perm \\
\hline 226 & $1.97 E+00$ & $9.85 E-03$ & perm \\
\hline 225.3 & $1.36 E+01$ & $6.81 \mathrm{E}-02$ & perm \\
\hline 224 & $1.35 \mathrm{E}+01$ & $6.76 \mathrm{E}-02$ & perm \\
\hline 223.3 & $1.72 E+01$ & $8.60 E-02$ & perm \\
\hline 222.3 & $1.82 \mathrm{E}+01$ & $9.09 E-02$ & perm \\
\hline 220.8 & $1.41 E+01$ & $3.92 E+00$ & $\mathrm{cpt}$ \\
\hline 219.3 & $4.64 \mathrm{E}+00$ & $2.32 E-02$ & perm \\
\hline 218.3 & $1.31 E+01$ & $6.56 \mathrm{E}-02$ & perm \\
\hline 217.3 & $1.18 \mathrm{E}+01$ & $5.92 \mathrm{E}-02$ & perm \\
\hline 216.3 & $1.57 E+01$ & $7.83 E-02$ & perm \\
\hline 215.3 & $1.66 E+01$ & $8.32 E-02$ & perm \\
\hline 214.3 & $1.20 \mathrm{E}+01$ & $5.99 \mathrm{E}-02$ & perm \\
\hline 213.3 & $5.73 \mathrm{E}+00$ & 2.87E-02 & perm \\
\hline 212.3 & $3.55 E+00$ & $1.78 E-02$ & perm \\
\hline 211.3 & $8.68 \mathrm{E}+00$ & $4.34 \mathrm{E}-02$ & perm \\
\hline 209.8 & $2.00 \mathrm{E}+01$ & $5.55 E+00$ & $\mathrm{cpt}$ \\
\hline 208.3 & $5.31 \mathrm{E}+00$ & $2.66 \mathrm{E}-02$ & perm \\
\hline 206.8 & $5.05 E+01$ & $1.40 E+01$ & core \\
\hline 205.8 & $4.60 \mathrm{E}+01$ & $1.28 E+01$ & core \\
\hline 204.3 & $1.23 \mathrm{E}+01$ & $6.13 E-02$ & perm \\
\hline 202.8 & $5.05 E+01$ & $1.40 E+01$ & core \\
\hline 201.8 & $5.05 E+01$ & $1.40 E+01$ & core \\
\hline 200.8 & $4.19 \mathrm{E}+01$ & $1.16 \mathrm{E}+01$ & core \\
\hline 199.8 & $5.05 E+01$ & $1.40 E+01$ & core \\
\hline
\end{tabular}


Conductivity Data at RPC1CL

\begin{tabular}{|c|c|c|c|}
\hline $\begin{array}{c}\text { Elevation } \\
\text { (ft) }\end{array}$ & $\begin{array}{c}\text { Kh } \\
\text { (ft/day) }\end{array}$ & $\begin{array}{c}\mathrm{Kv} \\
\text { (ft/day) }\end{array}$ & $\begin{array}{c}\text { Data } \\
\text { Source }\end{array}$ \\
\hline 198.8 & $5.54 \mathrm{E}+01$ & $1.54 E+01$ & core \\
\hline 197.3 & $9.21 E+00$ & $4.61 \mathrm{E}-02$ & perm \\
\hline 196 & $4.18 E+01$ & $2.09 \mathrm{E}-01$ & perm \\
\hline 195.3 & $4.29 E+01$ & $2.14 \mathrm{E}-01$ & perm \\
\hline 193.8 & $5.05 E+01$ & $1.40 E+01$ & core \\
\hline 192.8 & $5.05 E+01$ & $1.40 E+01$ & core \\
\hline 191.8 & $4.60 \mathrm{E}+01$ & $1.28 \mathrm{E}+01$ & core \\
\hline 190.8 & $3.82 \mathrm{E}+01$ & $1.06 \mathrm{E}+01$ & core \\
\hline 189.8 & $3.48 E+01$ & $9.67 E+00$ & core \\
\hline 188.8 & $6.07 E+01$ & $1.69 E+01$ & core \\
\hline 187.8 & $6.07 E+01$ & $1.69 \mathrm{E}+01$ & core \\
\hline 186.8 & $5.54 \mathrm{E}+01$ & $1.54 \mathrm{E}+01$ & core \\
\hline 185.8 & $4.19 E+01$ & $1.16 \mathrm{E}+01$ & core \\
\hline 184.8 & 4.19E+01 & $1.16 E+01$ & core \\
\hline 184.1 & $4.37 \mathrm{E}+01$ & $2.19 \mathrm{E}-01$ & perm \\
\hline 182.8 & $4.19 \mathrm{E}+01$ & $1.16 \mathrm{E}+01$ & core \\
\hline 181.8 & $3.48 E+01$ & $9.67 E+00$ & core \\
\hline 180.8 & $9.30 \mathrm{E}-04$ & $2.60 \mathrm{E}-04$ & $\mathrm{cpt}$ \\
\hline 179.8 & $5.30 \mathrm{E}-04$ & $1.50 \mathrm{E}-04$ & $\mathrm{cpt}$ \\
\hline 178.8 & $1.15 \mathrm{E}-04$ & $3.20 \mathrm{E}-05$ & core \\
\hline 177.3 & $4.07 \mathrm{E}+00$ & $1.13 \mathrm{E}+00$ & perm \\
\hline 177.1 & $5.62 E+01$ & 2.81E-01 & perm \\
\hline 176.3 & $7.74 \mathrm{E}-01$ & $2.15 \mathrm{E}-01$ & perm \\
\hline 176.3 & $6.03 \mathrm{E}+01$ & $3.01 \mathrm{E}-01$ & perm \\
\hline 175.3 & $4.79 \mathrm{E}+01$ & $2.40 \mathrm{E}-01$ & perm \\
\hline 173.8 & 1.39E-01 & $3.86 \mathrm{E}-02$ & core \\
\hline 172.3 & $6.50 \mathrm{E}+01$ & $3.25 \mathrm{E}-01$ & perm \\
\hline 171.3 & $5.01 \mathrm{E}+01$ & $2.50 \mathrm{E}-01$ & perm \\
\hline 170.3 & $4.26 \mathrm{E}+01$ & $2.13 E-01$ & perm \\
\hline 169.3 & $5.23 \mathrm{E}+01$ & $2.62 E-01$ & perm \\
\hline 167.8 & $3.76 \mathrm{E}+01$ & $1.88 \mathrm{E}-01$ & perm \\
\hline 166.8 & $7.87 \mathrm{E}+00$ & $3.94 \mathrm{E}-02$ & perm \\
\hline 166.3 & $1.91 \mathrm{E}+01$ & $9.55 \mathrm{E}-02$ & perm \\
\hline 164.8 & $2.54 \mathrm{E}-03$ & $7.05 E-04$ & core \\
\hline 163.8 & $1.82 \mathrm{E}+01$ & $5.06 E+00$ & core \\
\hline 162.8 & $2.89 E+01$ & $8.04 E+00$ & core \\
\hline 161.8 & $2.54 \mathrm{E}-03$ & $7.05 E-04$ & core \\
\hline 160.8 & 1.39E-01 & $3.86 \mathrm{E}-02$ & core \\
\hline 159.8 & $1.18 \mathrm{E}+01$ & $3.28 \mathrm{E}+00$ & $\mathrm{cpt}$ \\
\hline 158.3 & $6.62 \mathrm{E}-03$ & $1.84 \mathrm{E}-03$ & perm \\
\hline 156.8 & $2.72 \mathrm{E}-05$ & $7.56 \mathrm{E}-06$ & core \\
\hline 155.8 & $4.60 E+01$ & $1.28 \mathrm{E}+01$ & core \\
\hline 155.1 & $8.26 E+01$ & $4.13 E-01$ & perm \\
\hline 153.8 & $4.60 \mathrm{E}+01$ & $1.28 \mathrm{E}+01$ & core \\
\hline 152.8 & $3.83 E+01$ & $1.06 \mathrm{E}+01$ & core \\
\hline 151.8 & $2.90 E+01$ & $8.05 E+00$ & core \\
\hline 150.8 & $5.04 \mathrm{E}+01$ & $1.40 \mathrm{E}+01$ & perm \\
\hline 149.8 & $1.31 \mathrm{E}+01$ & $3.64 \mathrm{E}+00$ & cpt \\
\hline
\end{tabular}


Conductivity Data at RPC1CL

\begin{tabular}{|c|c|c|c|}
\hline $\begin{array}{c}\text { Elevation } \\
\text { (ft) }\end{array}$ & $\begin{array}{c}\text { Kh } \\
\text { (ft/day) }\end{array}$ & $\begin{array}{c}\mathrm{Kv} \\
\text { (ft/day) }\end{array}$ & $\begin{array}{c}\text { Data } \\
\text { Source }\end{array}$ \\
\hline 149 & $2.91 E+01$ & $8.08 E+00$ & perm \\
\hline 148.1 & $6.28 E+01$ & $1.75 E+01$ & perm \\
\hline 146.8 & $5.05 E+01$ & $1.40 \mathrm{E}+01$ & core \\
\hline 146 & $2.01 \mathrm{E}+01$ & $5.59 \mathrm{E}+00$ & perm \\
\hline 144.8 & $2.90 E+01$ & $8.05 E+00$ & core \\
\hline 143.8 & $4.78 E+01$ & $1.33 \mathrm{E}+01$ & perm \\
\hline 143.1 & $1.91 \mathrm{E}+01$ & $5.31 \mathrm{E}+00$ & perm \\
\hline 141.8 & $3.20 E+01$ & $8.89 E+00$ & perm \\
\hline 141.3 & $3.57 E+01$ & $9.92 \mathrm{E}+00$ & perm \\
\hline 140.3 & $4.71 E+01$ & $1.31 \mathrm{E}+01$ & perm \\
\hline 139 & $3.48 E+01$ & $9.67 \mathrm{E}+00$ & perm \\
\hline 138.3 & $3.99 E+01$ & $1.11 \mathrm{E}+01$ & perm \\
\hline 136.8 & $3.04 \mathrm{E}+01$ & $8.43 E+00$ & core \\
\hline 135.8 & $4.35 \mathrm{E}+01$ & $1.21 \mathrm{E}+01$ & core \\
\hline 134.8 & $5.44 \mathrm{E}+01$ & $1.51 \mathrm{E}+01$ & core \\
\hline 133.8 & $4.20 \mathrm{E}+01$ & $1.17 E+01$ & core \\
\hline 132.8 & $4.04 \mathrm{E}+01$ & $1.12 \mathrm{E}+01$ & core \\
\hline 131.8 & $3.76 \mathrm{E}+01$ & $1.04 E+01$ & core \\
\hline 130.8 & $1.24 \mathrm{E}+01$ & $3.44 \mathrm{E}+00$ & core \\
\hline 129.8 & $3.55 \mathrm{E}+01$ & $9.87 E+00$ & core \\
\hline 128.8 & $7.10 \mathrm{E}+00$ & $1.97 \mathrm{E}+00$ & core \\
\hline 127.8 & $1.52 \mathrm{E}+01$ & $4.21 \mathrm{E}+00$ & core \\
\hline 126.8 & $6.35 \mathrm{E}+00$ & $1.76 \mathrm{E}+00$ & core \\
\hline 125.8 & $7.64 E+00$ & $2.12 \mathrm{E}+00$ & core \\
\hline 124.8 & $2.87 E+01$ & $7.97 \mathrm{E}+00$ & core \\
\hline 123.8 & $5.34 E+01$ & $1.48 \mathrm{E}+01$ & core \\
\hline 122.8 & $5.34 \mathrm{E}+01$ & $1.48 \mathrm{E}+01$ & core \\
\hline 121.8 & $4.16 E+01$ & $1.16 \mathrm{E}+01$ & core \\
\hline 120.8 & $2.52 E+01$ & $7.01 \mathrm{E}+00$ & core \\
\hline 119.8 & $6.19 E+01$ & $1.72 \mathrm{E}+01$ & core \\
\hline 118.8 & $6.31 E+01$ & $1.75 \mathrm{E}+01$ & core \\
\hline 117.8 & $5.14 E+01$ & $1.43 \mathrm{E}+01$ & core \\
\hline 116.8 & $4.69 \mathrm{E}+01$ & $1.30 \mathrm{E}+01$ & core \\
\hline 115.8 & $3.97 E+01$ & $1.10 \mathrm{E}+01$ & core \\
\hline 113.8 & $5.44 \mathrm{E}+01$ & $1.51 \mathrm{E}+01$ & core \\
\hline 112.8 & $4.65 E+01$ & $1.29 E+01$ & core \\
\hline 111.8 & $5.00 \mathrm{E}+01$ & $1.39 \mathrm{E}+01$ & core \\
\hline 110.8 & $5.97 E+01$ & $1.66 E+01$ & core \\
\hline 108.8 & $2.95 \mathrm{E}+01$ & $8.20 \mathrm{E}+00$ & core \\
\hline 107.8 & $4.12 E+01$ & $1.14 \mathrm{E}+01$ & core \\
\hline 106.8 & $3.93 \mathrm{E}+01$ & $1.09 \mathrm{E}+01$ & core \\
\hline 105.8 & $3.27 \mathrm{E}+01$ & $9.08 \mathrm{E}+00$ & core \\
\hline 103.8 & $3.15 E+01$ & $8.75 \mathrm{E}+00$ & core \\
\hline 102.8 & $3.42 \mathrm{E}+01$ & $9.51 \mathrm{E}+00$ & core \\
\hline 101.8 & $3.76 \mathrm{E}+01$ & $1.04 \mathrm{E}+01$ & core \\
\hline 100.8 & $3.86 \mathrm{E}+01$ & $1.07 \mathrm{E}+01$ & core \\
\hline 99.8 & $5.44 \mathrm{E}+01$ & $1.51 \mathrm{E}+01$ & core \\
\hline 96.8 & $2.10 \mathrm{E}+01$ & $5.82 \mathrm{E}+00$ & core \\
\hline
\end{tabular}


Conductivity Data at RPC1CL

\begin{tabular}{|c|c|c|c|}
\hline $\begin{array}{c}\text { Elevation } \\
\text { (ft) }\end{array}$ & $\begin{array}{c}\text { Kh } \\
\text { (ft/day) }\end{array}$ & $\begin{array}{c}\text { Kv } \\
\text { (ft/day) }\end{array}$ & $\begin{array}{c}\text { Data } \\
\text { Source }\end{array}$ \\
\hline 94.8 & $5.08 \mathrm{E}+00$ & $1.41 \mathrm{E}+00$ & core \\
\hline 93.8 & $5.00 \mathrm{E}+01$ & $1.39 \mathrm{E}+01$ & core \\
\hline 92.3 & $1.43 \mathrm{E}-03$ & $3.97 \mathrm{E}-04$ & perm \\
\hline
\end{tabular}


Conductivity Data at RPC2CL

\begin{tabular}{|c|c|c|c|}
\hline $\begin{array}{c}\text { Elevation } \\
\text { (ft) }\end{array}$ & $\begin{array}{c}\text { Kh } \\
\text { (ft/day) }\end{array}$ & $\begin{array}{c}\text { Kv } \\
\text { (ft/day) }\end{array}$ & $\begin{array}{c}\text { Data } \\
\text { Source }\end{array}$ \\
\hline 291.8 & $4.72 \mathrm{E}+00$ & $1.31 \mathrm{E}+00$ & cpt \\
\hline 290.8 & $5.34 \mathrm{E}+00$ & $1.48 \mathrm{E}+00$ & cpt \\
\hline 289.8 & $4.82 \mathrm{E}+00$ & $1.34 \mathrm{E}+00$ & cpt \\
\hline 288.8 & $4.60 \mathrm{E}+00$ & $1.28 \mathrm{E}+00$ & cpt \\
\hline 287.8 & $5.42 \mathrm{E}+00$ & $1.51 \mathrm{E}+00$ & cpt \\
\hline 286.8 & $5.77 \mathrm{E}+00$ & $1.60 \mathrm{E}+00$ & cpt \\
\hline 285.8 & $1.05 \mathrm{E}+01$ & $2.93 \mathrm{E}+00$ & cpt \\
\hline 284.8 & $2.79 \mathrm{E}+00$ & $7.74 \mathrm{E}-01$ & cpt \\
\hline 283.8 & $3.57 \mathrm{E}-02$ & $9.92 \mathrm{E}-03$ & perm \\
\hline 282.8 & $4.04 \mathrm{E}-02$ & $1.12 \mathrm{E}-02$ & cpt \\
\hline 281.8 & $2.04 \mathrm{E}-01$ & $5.68 \mathrm{E}-02$ & cpt \\
\hline 280.8 & $6.96 \mathrm{E}-01$ & $1.93 \mathrm{E}-01$ & cpt \\
\hline 279.8 & $7.27 \mathrm{E}+00$ & $2.02 \mathrm{E}+00$ & cpt \\
\hline 278.3 & $3.69 \mathrm{E}-05$ & $8.22 \mathrm{E}-05$ & perm \\
\hline 276.3 & $6.53 \mathrm{E}+00$ & $1.81 \mathrm{E}+00$ & perm \\
\hline 274.6 & $3.64 \mathrm{E}+00$ & $1.01 \mathrm{E}+00$ & perm \\
\hline 273.9 & $1.09 \mathrm{E}+01$ & $3.02 \mathrm{E}+00$ & perm \\
\hline 273.1 & $1.17 \mathrm{E}+01$ & $3.25 \mathrm{E}+00$ & perm \\
\hline 272.1 & $7.74 \mathrm{E}+00$ & $2.15 \mathrm{E}+00$ & perm \\
\hline 271.3 & $6.14 \mathrm{E}+00$ & $1.71 \mathrm{E}+00$ & perm \\
\hline 269.7 & $9.04 \mathrm{E}+00$ & $2.51 \mathrm{E}+00$ & perm \\
\hline 268.8 & $7.24 \mathrm{E}+00$ & $2.01 \mathrm{E}+00$ & core \\
\hline 267.8 & $1.15 \mathrm{E}+01$ & $3.19 \mathrm{E}+00$ & core \\
\hline 266.8 & $1.81 \mathrm{E}+00$ & $5.02 \mathrm{E}-01$ & core \\
\hline 265.8 & $1.14 \mathrm{E}+00$ & $3.16 \mathrm{E}-01$ & core \\
\hline 264.8 & $1.81 \mathrm{E}+00$ & $5.02 \mathrm{E}-01$ & core \\
\hline 263.8 & $1.43 \mathrm{E}-03$ & $4.00 \mathrm{E}-04$ & cpt \\
\hline 262.8 & $1.14 \mathrm{E}+00$ & $3.16 \mathrm{E}-01$ & core \\
\hline 261.8 & $1.81 \mathrm{E}+00$ & $5.02 \mathrm{E}-01$ & core \\
\hline 260.8 & $1.14 \mathrm{E}+00$ & $3.16 \mathrm{E}-01$ & core \\
\hline 259.8 & $6.40 \mathrm{E}-04$ & $1.80 \mathrm{E}-04$ & cpt \\
\hline 258.8 & $5.50 \mathrm{E}-04$ & $1.50 \mathrm{E}-04$ & cpt \\
\hline 257.3 & $1.47 \mathrm{E}-02$ & $2.27 \mathrm{E}-03$ & perm \\
\hline 255.8 & $2.87 \mathrm{E}+00$ & $7.97 \mathrm{E}-01$ & core \\
\hline 254.8 & $1.81 \mathrm{E}+00$ & $5.02 \mathrm{E}-01$ & core \\
\hline 253.8 & $5.40 \mathrm{E}-04$ & $1.50 \mathrm{E}-04$ & cpt \\
\hline 252.3 & $1.03 \mathrm{E}+01$ & $1.03 \mathrm{E}-01$ & perm \\
\hline 250.8 & $6.20 \mathrm{E}-04$ & $1.70 \mathrm{E}-04$ & cpt \\
\hline 249.8 & $7.60 \mathrm{E}-04$ & $2.10 \mathrm{E}-04$ & cpt \\
\hline 248.3 & $8.10 \mathrm{E}+00$ & $8.10 \mathrm{E}-02$ & perm \\
\hline 247.3 & $1.36 \mathrm{E}+01$ & $1.36 \mathrm{E}-01$ & perm \\
\hline 246.3 & $5.21 \mathrm{E}+00$ & $5.21 \mathrm{E}-02$ & perm \\
\hline 234.8 & $7.24 \mathrm{E}+00$ & $2.01 \mathrm{E}+00$ & core \\
\hline $2.69 \mathrm{E}+01$ & $2.69 \mathrm{E}-01$ & perm \\
\hline $1.96 \mathrm{E}+00$ & $1.96 \mathrm{E}-02$ & perm \\
\hline 23.8 & $5.65 \mathrm{E}+00$ & $1.57 \mathrm{E}+00$ & cpt \\
\hline $6.13 \mathrm{E}-01$ & $1.70 \mathrm{E}-01$ & cpt \\
\hline
\end{tabular}


Conductivity Data at RPC2CL

\begin{tabular}{|c|c|c|c|}
\hline $\begin{array}{c}\text { Elevation } \\
\text { (ft) }\end{array}$ & $\begin{array}{c}\text { Kh } \\
\text { (ft/day) }\end{array}$ & $\begin{array}{c}\text { Kv } \\
\text { (ft'day) }\end{array}$ & $\begin{array}{l}\text { Data } \\
\text { Source }\end{array}$ \\
\hline 237.8 & $7.24 \mathrm{E}+00$ & $2.01 \mathrm{E}+00$ & core \\
\hline 236.8 & $1.15 E+01$ & $3.19 \mathrm{E}+00$ & core \\
\hline 235.3 & $3.79 E+00$ & $3.79 E-02$ & perm \\
\hline 234.3 & $1.92 E+00$ & $1.92 \mathrm{E}-02$ & perm \\
\hline 232.9 & $2.78 E+00$ & $2.78 \mathrm{E}-02$ & perm \\
\hline 231.9 & $7.37 \mathrm{E}+00$ & 7.37E-02 & perm \\
\hline 230.3 & $3.78 E+00$ & $3.78 \mathrm{E}-02$ & perm \\
\hline 229.3 & $2.09 E+01$ & $2.09 E-01$ & perm \\
\hline 228.3 & $8.88 E+00$ & $8.88 \mathrm{E}-02$ & perm \\
\hline 227.3 & $7.85 E+00$ & $7.85 E-02$ & perm \\
\hline 226.3 & $4.66 E+00$ & 4.66E-02 & perm \\
\hline 224.8 & $1.15 E+01$ & $3.19 E+00$ & core \\
\hline 223.8 & $1.15 E+01$ & $3.19 E+00$ & core \\
\hline 222.3 & $3.38 \mathrm{E}+00$ & $3.38 \mathrm{E}-02$ & perm \\
\hline 220.3 & $4.28 E+00$ & $4.28 \mathrm{E}-02$ & perm \\
\hline 218.9 & $2.35 E+00$ & $2.35 \mathrm{E}-02$ & perm \\
\hline 217.3 & $7.42 \mathrm{E}+00$ & 7.42E-02 & perm \\
\hline 216.0 & $6.48 E+00$ & $6.48 \mathrm{E}-02$ & perm \\
\hline 215.0 & $3.77 E+00$ & $1.89 \mathrm{E}-02$ & perm \\
\hline 214.3 & $1.50 \mathrm{E}+01$ & $7.49 \mathrm{E}-02$ & perm \\
\hline 213.3 & $1.73 E+01$ & $8.63 \mathrm{E}-02$ & perm \\
\hline 212.3 & $3.02 E+01$ & $1.51 \mathrm{E}-01$ & perm \\
\hline 211.3 & $2.58 \mathrm{E}+01$ & $1.29 \mathrm{E}-01$ & perm \\
\hline 210.3 & $2.30 \mathrm{E}+01$ & $1.15 \mathrm{E}-01$ & perm \\
\hline 209.3 & $1.36 \mathrm{E}+01$ & $6.81 \mathrm{E}-02$ & perm \\
\hline 208.0 & $2.87 \mathrm{E}+01$ & $1.43 E-01$ & perm \\
\hline 207.0 & $3.10 \mathrm{E}+01$ & $1.55 \mathrm{E}-01$ & perm \\
\hline 206.3 & $2.95 E+01$ & $1.47 \mathrm{E}-01$ & perm \\
\hline 205.3 & $1.03 E+01$ & $5.16 \mathrm{E}-02$ & perm \\
\hline 204.1 & $7.32 E+00$ & $3.66 \mathrm{E}-02$ & perm \\
\hline 202.8 & $1.00 \mathrm{E}-03$ & $2.80 \mathrm{E}-04$ & cpt \\
\hline 201.8 & $1.33 \mathrm{E}-03$ & $3.70 \mathrm{E}-04$ & $\mathrm{cpt}$ \\
\hline 200.8 & $1.76 \mathrm{E}+00$ & $4.90 \mathrm{E}-01$ & $\mathrm{cpt}$ \\
\hline 199.8 & $4.82 \mathrm{E}+01$ & $1.34 E+01$ & $\mathrm{cpt}$ \\
\hline 198.8 & $6.97 E+01$ & $1.94 \mathrm{E}+01$ & $\mathrm{cpt}$ \\
\hline 197.8 & $5.82 E+01$ & $1.62 \mathrm{E}+01$ & $\mathrm{cpt}$ \\
\hline 196.8 & $5.11 \mathrm{E}+01$ & $1.42 \mathrm{E}+01$ & $\mathrm{cpt}$ \\
\hline 195.8 & $3.12 E+01$ & $8.68 \mathrm{E}+00$ & cpt \\
\hline 194.8 & $1.30 \mathrm{E}+01$ & $3.61 \mathrm{E}+00$ & cpt \\
\hline 193.8 & $1.34 \mathrm{E}+00$ & $3.72 \mathrm{E}-01$ & cpt \\
\hline 192.8 & $4.40 \mathrm{E}-04$ & $1.20 \mathrm{E}-04$ & $\mathrm{cpt}$ \\
\hline 191.8 & $1.84 \mathrm{E}+01$ & $5.12 \mathrm{E}+00$ & $\mathrm{cpt}$ \\
\hline 190.8 & $2.61 \mathrm{E}-01$ & $7.26 \mathrm{E}-02$ & $\overline{c p t}$ \\
\hline 189.8 & $1.32 \mathrm{E}+00$ & $3.66 \mathrm{E}-01$ & cpt \\
\hline 188.8 & $3.70 \mathrm{E}-01$ & $1.03 E-01$ & cpt \\
\hline 187.8 & $2.53 E-02$ & $7.02 \mathrm{E}-03$ & $\mathrm{cpt}$ \\
\hline 186.8 & $3.43 \mathrm{E}-03$ & $9.50 \mathrm{E}-04$ & $\mathrm{cpt}$ \\
\hline 185.8 & $3.83 \mathrm{E}-03$ & $1.06 \mathrm{E}-03$ & cpt \\
\hline
\end{tabular}


Conductivity Data at RPC2CL

\begin{tabular}{|c|c|c|c|}
\hline $\begin{array}{c}\text { Elevation } \\
\text { (ft) }\end{array}$ & $\begin{array}{c}\text { Kh } \\
\text { (ft/day) }\end{array}$ & $\begin{array}{c}\text { Kv } \\
\text { (ft/day) }\end{array}$ & $\begin{array}{c}\text { Data } \\
\text { Source }\end{array}$ \\
\hline 184.8 & $1.61 \mathrm{E}-03$ & $4.50 \mathrm{E}-04$ & cpt \\
\hline 183.8 & $4.26 \mathrm{E}-03$ & $1.18 \mathrm{E}-03$ & $\mathrm{cpt}$ \\
\hline 182.8 & $5.46 \mathrm{E}-03$ & $1.52 \mathrm{E}-03$ & cpt \\
\hline 181.8 & $1.49 \mathrm{E}-02$ & $4.14 \mathrm{E}-03$ & cpt \\
\hline 180.8 & $4.43 \mathrm{E}-03$ & $1.23 \mathrm{E}-03$ & cpt \\
\hline 179.8 & $7.96 \mathrm{E}-03$ & $2.21 \mathrm{E}-03$ & cpt \\
\hline 178.8 & $1.23 \mathrm{E}+01$ & $3.42 \mathrm{E}+00$ & cpt \\
\hline 177.9 & $6.15 \mathrm{E}+01$ & $3.08 \mathrm{E}-01$ & perm \\
\hline 176.3 & $6.23 \mathrm{E}+01$ & $3.11 \mathrm{E}-01$ & perm \\
\hline 175.3 & $4.56 \mathrm{E}+01$ & $2.28 \mathrm{E}-01$ & perm \\
\hline 173.8 & $1.18 \mathrm{E}-03$ & $3.30 \mathrm{E}-04$ & cpt \\
\hline 172.8 & $1.35 \mathrm{E}+01$ & $6.76 \mathrm{E}-02$ & perm \\
\hline 172.3 & $4.16 \mathrm{E}+01$ & $2.08 \mathrm{E}-01$ & perm \\
\hline 171.3 & $5.02 \mathrm{E}+01$ & $2.51 \mathrm{E}-01$ & perm \\
\hline 170.3 & $4.82 \mathrm{E}+01$ & $2.41 \mathrm{E}-01$ & perm \\
\hline 169.3 & $4.79 \mathrm{E}+01$ & $2.40 \mathrm{E}-01$ & perm \\
\hline 168.3 & $5.74 \mathrm{E}+01$ & $2.87 \mathrm{E}-01$ & perm \\
\hline 167.3 & $4.51 \mathrm{E}+01$ & $2.25 \mathrm{E}-01$ & perm \\
\hline 166.3 & $6.57 \mathrm{E}+01$ & $3.29 \mathrm{E}-01$ & perm \\
\hline 165.3 & $6.90 \mathrm{E}+01$ & $3.45 \mathrm{E}-01$ & perm \\
\hline 164.3 & $6.03 \mathrm{E}+01$ & $3.01 \mathrm{E}-01$ & perm \\
\hline 162.7 & $3.02 \mathrm{E}+01$ & $1.51 \mathrm{E}-01$ & perm \\
\hline 162.3 & $1.38 \mathrm{E}+01$ & $6.91 \mathrm{E}-02$ & perm \\
\hline 161.3 & $3.22 \mathrm{E}+01$ & $1.61 \mathrm{E}-01$ & perm \\
\hline 159.8 & $2.89 \mathrm{E}+01$ & $8.04 \mathrm{E}+00$ & core \\
\hline 158.3 & $4.65 \mathrm{E}+01$ & $2.33 \mathrm{E}-01$ & perm \\
\hline 157.3 & $5.30 \mathrm{E}+01$ & $2.65 \mathrm{E}-01$ & perm \\
\hline 156.3 & $5.70 \mathrm{E}+01$ & $2.85 \mathrm{E}-01$ & perm \\
\hline 155.3 & $4.82 \mathrm{E}+01$ & $2.41 \mathrm{E}-01$ & perm \\
\hline 154.3 & $5.23 \mathrm{E}+01$ & $2.62 \mathrm{E}-01$ & perm \\
\hline 153.0 & $5.79 \mathrm{E}+01$ & $2.89 \mathrm{E}-01$ & perm \\
\hline 152.3 & $5.79 \mathrm{E}+01$ & $2.89 \mathrm{E}-01$ & perm \\
\hline 150.8 & $4.69 \mathrm{E}+01$ & $1.30 \mathrm{E}+01$ & cpt \\
\hline 149.8 & $5.83 \mathrm{E}+01$ & $1.62 \mathrm{E}+01$ & cpt \\
\hline 148.8 & $6.12 \mathrm{E}+01$ & $1.70 \mathrm{E}+01$ & cpt \\
\hline 147.3 & $5.08 \mathrm{E}+01$ & $1.41 \mathrm{E}+01$ & perm \\
\hline 146.3 & $6.53 \mathrm{E}+01$ & $1.81 \mathrm{E}+01$ & perm \\
\hline 145.3 & $5.74 \mathrm{E}+01$ & $1.59 \mathrm{E}+01$ & perm \\
\hline 144.3 & $5.83 \mathrm{E}+01$ & $1.62 \mathrm{E}+01$ & perm \\
\hline 143.1 & $5.36 \mathrm{E}+01$ & $1.49 \mathrm{E}+01$ & perm \\
\hline 142.3 & $5.49 \mathrm{E}+01$ & $1.53 \mathrm{E}+01$ & perm \\
\hline 141.3 & $5.07 \mathrm{E}+01$ & $1.41 \mathrm{E}+01$ & perm \\
\hline 140.3 & $5.27 \mathrm{E}+01$ & $1.46 \mathrm{E}+01$ & perm \\
\hline 139.3 & $6.59 \mathrm{E}+01$ & $1.83 \mathrm{E}+01$ & perm \\
\hline 137.0 & $1.05 \mathrm{E}+02$ & $2.90 \mathrm{E}+01$ & perm \\
\hline 136.3 & $8.18 \mathrm{E}+01$ & $2.27 \mathrm{E}+01$ & perm \\
\hline 133.3 & $1.04 \mathrm{E}+02$ & $2.89 \mathrm{E}+01$ & perm \\
\hline & $8.30 \mathrm{E}+01$ & $2.31 \mathrm{E}+01$ & perm \\
\hline
\end{tabular}


Conductivity Data at RPC2CL

\begin{tabular}{|c|c|c|c|}
\hline $\begin{array}{c}\text { Elevation } \\
\text { (ft) }\end{array}$ & $\begin{array}{c}\mathrm{Kh} \\
\text { (ft/day) }\end{array}$ & $\begin{array}{c}\text { Kv } \\
\text { (ft/day) }\end{array}$ & $\begin{array}{c}\text { Data } \\
\text { Source }\end{array}$ \\
\hline 132.3 & $7.80 \mathrm{E}+01$ & $2.17 \mathrm{E}+01$ & perm \\
\hline 131.3 & $7.95 \mathrm{E}+01$ & $2.21 E+01$ & perm \\
\hline 130.0 & $7.84 E+01$ & $2.18 \mathrm{E}+01$ & perm \\
\hline 129.3 & $5.47 \mathrm{E}+01$ & $1.52 \mathrm{E}+01$ & perm \\
\hline 128.3 & $8.39 \mathrm{E}+01$ & $2.33 \mathrm{E}+01$ & perm \\
\hline 127.3 & $5.86 \mathrm{E}+01$ & $1.63 \mathrm{E}+01$ & perm \\
\hline 126.3 & $5.25 \mathrm{E}+01$ & $1.46 \mathrm{E}+01$ & perm \\
\hline 125.3 & $3.13 E+01$ & $8.68 \mathrm{E}+00$ & perm \\
\hline 123.8 & $7.23 \mathrm{E}+00$ & $2.01 \mathrm{E}+00$ & core \\
\hline 122.8 & $2.04 E+01$ & $5.65 \mathrm{E}+00$ & perm \\
\hline 121.3 & $2.21 \mathrm{E}+01$ & $6.14 \mathrm{E}+00$ & perm \\
\hline 120.3 & $3.31 \mathrm{E}+01$ & $9.18 \mathrm{E}+00$ & perm \\
\hline 118.3 & $2.70 E+01$ & $7.51 \mathrm{E}+00$ & perm \\
\hline 117.3 & $2.78 \mathrm{E}+01$ & $7.73 \mathrm{E}+00$ & perm \\
\hline 115.8 & $1.72 E+01$ & $4.77 \mathrm{E}+00$ & perm \\
\hline 115.3 & $2.25 E+01$ & $6.26 E+00$ & perm \\
\hline 113.3 & $4.78 \mathrm{E}+01$ & $1.33 E+01$ & perm \\
\hline 112.3 & $3.88 \mathrm{E}+01$ & $1.08 E+01$ & perm \\
\hline 103.3 & $1.77 \mathrm{E}+01$ & $4.91 \mathrm{E}+00$ & perm \\
\hline 102.3 & $2.48 \mathrm{E}+01$ & $6.88 \mathrm{E}+00$ & perm \\
\hline 100.8 & $1.91 \mathrm{E}+01$ & $5.31 \mathrm{E}+00$ & core \\
\hline 99.8 & $4.20 \mathrm{E}+01$ & $1.17 \mathrm{E}+01$ & core \\
\hline 98.8 & $3.49 E+01$ & $9.69 \mathrm{E}+00$ & core \\
\hline 97.8 & $3.79 E+01$ & $1.05 E+01$ & core \\
\hline 96.8 & $1.49 E+01$ & $4.13 E+00$ & core \\
\hline 95.8 & $1.82 \mathrm{E}+01$ & $5.07 \mathrm{E}+00$ & core \\
\hline 94.8 & $4.15 E+00$ & $1.15 \mathrm{E}+00$ & core \\
\hline 93.8 & $1.10 E+01$ & $3.05 E+00$ & core \\
\hline 92.8 & $4.55 E+00$ & $1.26 \mathrm{E}+00$ & core \\
\hline 91.8 & $2.57 \mathrm{E}-05$ & $7.14 \mathrm{E}-06$ & core \\
\hline 90.8 & $2.57 \mathrm{E}-05$ & $7.14 E-06$ & core \\
\hline
\end{tabular}


Conductivity Data at RPT1

\begin{tabular}{|c|c|c|c|}
\hline $\begin{array}{c}\text { Elevation } \\
\text { (ft) }\end{array}$ & $\begin{array}{c}\text { Kh } \\
\text { (ft/day) }\end{array}$ & $\begin{array}{c}\text { Kv } \\
\text { (ft/day) }\end{array}$ & $\begin{array}{c}\text { Data } \\
\text { Source }\end{array}$ \\
\hline 289.8 & $3.28 \mathrm{E}+00$ & $9.12 \mathrm{E}-01$ & $\mathrm{cpt}$ \\
\hline 288.8 & $3.29 \mathrm{E}+00$ & $9.14 \mathrm{E}-01$ & $\mathrm{cpt}$ \\
\hline 287.8 & $3.39 \mathrm{E}+00$ & $9.41 \mathrm{E}-01$ & $\mathrm{cpt}$ \\
\hline 286.8 & $3.47 \mathrm{E}+00$ & $9.64 \mathrm{E}-01$ & $\mathrm{cpt}$ \\
\hline 285.8 & $3.50 \mathrm{E}+00$ & $9.73 \mathrm{E}-01$ & $\mathrm{cpt}$ \\
\hline 284.8 & $3.49 \mathrm{E}+00$ & $9.69 \mathrm{E}-01$ & $\mathrm{cpt}$ \\
\hline 283.8 & $3.97 \mathrm{E}+00$ & $1.10 \mathrm{E}+00$ & $\mathrm{cpt}$ \\
\hline 282.8 & $7.39 \mathrm{E}+00$ & $2.05 \mathrm{E}+00$ & $\mathrm{cpt}$ \\
\hline 281.8 & $9.59 \mathrm{E}+00$ & $2.66 \mathrm{E}+00$ & $\mathrm{cpt}$ \\
\hline 280.8 & $1.69 \mathrm{E}+01$ & $4.68 \mathrm{E}+00$ & $\mathrm{cpt}$ \\
\hline 279.8 & $2.88 \mathrm{E}+01$ & $7.99 \mathrm{E}+00$ & $\mathrm{cpt}$ \\
\hline 278.8 & $4.99 \mathrm{E}+01$ & $1.39 \mathrm{E}+01$ & $\mathrm{cpt}$ \\
\hline 277.8 & $4.67 \mathrm{E}+01$ & $1.30 \mathrm{E}+01$ & $\mathrm{cpt}$ \\
\hline 276.8 & $1.20 \mathrm{E}-01$ & $3.34 \mathrm{E}-02$ & $\mathrm{cpt}$ \\
\hline 275.8 & $1.04 \mathrm{E}-03$ & $2.90 \mathrm{E}-04$ & $\mathrm{cpt}$ \\
\hline 274.8 & $2.01 \mathrm{E}-01$ & $5.58 \mathrm{E}-02$ & $\mathrm{cpt}$ \\
\hline 273.8 & $7.22 \mathrm{E}-01$ & $2.00 \mathrm{E}-01$ & $\mathrm{cpt}$ \\
\hline 272.8 & $2.94 \mathrm{E}+00$ & $8.16 \mathrm{E}-01$ & $\mathrm{cpt}$ \\
\hline 271.8 & $1.38 \mathrm{E}-03$ & $3.80 \mathrm{E}-04$ & $\mathrm{cpt}$ \\
\hline 270.8 & $3.82 \mathrm{E}+01$ & $1.06 \mathrm{E}+01$ & $\mathrm{cpt}$ \\
\hline 269.8 & $4.98 \mathrm{E}+01$ & $1.38 \mathrm{E}+01$ & $\mathrm{cpt}$ \\
\hline 268.8 & $3.58 \mathrm{E}+00$ & $9.94 \mathrm{E}-01$ & $\mathrm{cpt}$ \\
\hline 267.8 & $1.23 \mathrm{E}-01$ & $3.41 \mathrm{E}-02$ & $\mathrm{cpt}$ \\
\hline 266.8 & $3.03 \mathrm{E}-01$ & $8.41 \mathrm{E}-02$ & $\mathrm{cpt}$ \\
\hline 265.8 & $8.23 \mathrm{E}-01$ & $2.29 \mathrm{E}-01$ & $\mathrm{cpt}$ \\
\hline 264.8 & $5.50 \mathrm{E}-03$ & $1.53 \mathrm{E}-03$ & $\mathrm{cpt}$ \\
\hline 263.8 & $1.48 \mathrm{E}-03$ & $4.10 \mathrm{E}-04$ & $\mathrm{cpt}$ \\
\hline 262.8 & $7.80 \mathrm{E}-04$ & $2.20 \mathrm{E}-04$ & $\mathrm{cpt}$ \\
\hline 261.8 & $1.06 \mathrm{E}+01$ & $2.94 \mathrm{E}+00$ & $\mathrm{cpt}$ \\
\hline 260.8 & $1.15 \mathrm{E}-03$ & $3.20 \mathrm{E}-04$ & $\mathrm{cpt}$ \\
\hline 259.8 & $7.00 \mathrm{E}-04$ & $1.90 \mathrm{E}-04$ & $\mathrm{cpt}$ \\
\hline 258.8 & $1.87 \mathrm{E}-03$ & $5.20 \mathrm{E}-04$ & $\mathrm{cpt}$ \\
\hline 257.8 & $3.28 \mathrm{E}-03$ & $9.10 \mathrm{E}-04$ & $\mathrm{cpt}$ \\
\hline 256.8 & $6.80 \mathrm{E}-04$ & $1.90 \mathrm{E}-04$ & $\mathrm{cpt}$ \\
\hline 255.8 & $5.90 \mathrm{E}-04$ & $1.60 \mathrm{E}-04$ & $\mathrm{cpt}$ \\
\hline 254.8 & $5.90 \mathrm{E}-04$ & $1.60 \mathrm{E}-04$ & $\mathrm{cpt}$ \\
\hline 253.8 & $6.20 \mathrm{E}-04$ & $1.70 \mathrm{E}-04$ & $\mathrm{cpt}$ \\
\hline 252.8 & $5.90 \mathrm{E}-04$ & $1.60 \mathrm{E}-04$ & $\mathrm{cpt}$ \\
\hline 251.8 & $5.70 \mathrm{E}-04$ & $1.60 \mathrm{E}-04$ & $\mathrm{cpt}$ \\
\hline 250.8 & $5.90 \mathrm{E}-04$ & $1.70 \mathrm{E}-04$ & $\mathrm{cpt}$ \\
\hline 249.8 & $7.70 \mathrm{E}+00$ & $2.14 \mathrm{E}+00$ & $\mathrm{cpt}$ \\
\hline 248.8 & $3.30 \mathrm{E}+00$ & $9.16 \mathrm{E}-01$ & $\mathrm{cpt}$ \\
\hline 247.8 & $6.23 \mathrm{E}+00$ & $1.73 \mathrm{E}+00$ & $\mathrm{cpt}$ \\
\hline 246.8 & $7.56 \mathrm{E}+00$ & $2.10 \mathrm{E}+00$ & $\mathrm{cpt}$ \\
\hline 243.8 & $5.38 \mathrm{E}+00$ & $1.50 \mathrm{E}+00$ & $\mathrm{cpt}$ \\
\hline 7.8 & $7.22 \mathrm{E}+00$ & $2.00 \mathrm{E}+00$ & $\mathrm{cpt}$ \\
\hline $5.84 \mathrm{E}+00$ & $1.62 \mathrm{E}+00$ & $\mathrm{cpt}$ \\
\hline $1.52 \mathrm{E}+01$ & $4.21 \mathrm{E}+00$ & $\mathrm{cpt}$ \\
\hline
\end{tabular}


Conductivity Data at RPT1

\begin{tabular}{|c|c|c|c|}
\hline $\begin{array}{c}\text { Elevation } \\
\text { (ft) }\end{array}$ & $\begin{array}{c}\text { Kh } \\
\text { (ft/day) }\end{array}$ & $\begin{array}{c}\text { Kv } \\
\text { (ft/day) }\end{array}$ & $\begin{array}{c}\text { Data } \\
\text { Source }\end{array}$ \\
\hline 241.8 & $9.89 \mathrm{E}+00$ & $2.75 \mathrm{E}+00$ & $\mathrm{cpt}$ \\
\hline 240.8 & $6.69 \mathrm{E}+00$ & $1.86 \mathrm{E}+00$ & $\mathrm{cpt}$ \\
\hline 239.8 & $6.69 \mathrm{E}+00$ & $1.86 \mathrm{E}+00$ & $\mathrm{cpt}$ \\
\hline 238.8 & $6.36 \mathrm{E}+00$ & $1.77 \mathrm{E}+00$ & $\mathrm{cpt}$ \\
\hline 237.8 & $2.48 \mathrm{E}-01$ & $6.89 \mathrm{E}-02$ & $\mathrm{cpt}$ \\
\hline 236.8 & $8.98 \mathrm{E}+00$ & $2.49 \mathrm{E}+00$ & $\mathrm{cpt}$ \\
\hline 235.8 & $1.01 \mathrm{E}+01$ & $2.81 \mathrm{E}+00$ & $\mathrm{cpt}$ \\
\hline 234.8 & $1.17 \mathrm{E}+01$ & $3.25 \mathrm{E}+00$ & $\mathrm{cpt}$ \\
\hline 233.8 & $1.31 \mathrm{E}+01$ & $3.64 \mathrm{E}+00$ & $\mathrm{cpt}$ \\
\hline 232.8 & $1.86 \mathrm{E}+01$ & $5.17 \mathrm{E}+00$ & $\mathrm{cpt}$ \\
\hline 231.8 & $1.38 \mathrm{E}+01$ & $3.85 \mathrm{E}+00$ & $\mathrm{cpt}$ \\
\hline 230.8 & $8.93 \mathrm{E}+00$ & $2.48 \mathrm{E}+00$ & $\mathrm{cpt}$ \\
\hline 229.8 & $2.00 \mathrm{E}+00$ & $5.55 \mathrm{E}-01$ & $\mathrm{cpt}$ \\
\hline 228.8 & $2.38 \mathrm{E}-01$ & $6.60 \mathrm{E}-02$ & $\mathrm{cpt}$ \\
\hline 227.8 & $5.05 \mathrm{E}+00$ & $1.40 \mathrm{E}+00$ & $\mathrm{cpt}$ \\
\hline 226.8 & $7.14 \mathrm{E}+00$ & $1.98 \mathrm{E}+00$ & $\mathrm{cpt}$ \\
\hline 225.8 & $7.60 \mathrm{E}+00$ & $2.11 \mathrm{E}+00$ & $\mathrm{cpt}$ \\
\hline 224.8 & $7.99 \mathrm{E}+00$ & $2.22 \mathrm{E}+00$ & $\mathrm{cpt}$ \\
\hline 223.8 & $9.29 \mathrm{E}+00$ & $2.58 \mathrm{E}+00$ & $\mathrm{cpt}$ \\
\hline 222.8 & $1.30 \mathrm{E}+00$ & $3.60 \mathrm{E}-01$ & $\mathrm{cpt}$ \\
\hline 221.8 & $1.26 \mathrm{E}-01$ & $3.49 \mathrm{E}-02$ & $\mathrm{cpt}$ \\
\hline 220.8 & $1.85 \mathrm{E}-01$ & $5.13 \mathrm{E}-02$ & $\mathrm{cpt}$ \\
\hline 219.8 & $4.95 \mathrm{E}+01$ & $1.38 \mathrm{E}+01$ & $\mathrm{cpt}$ \\
\hline 218.8 & $1.18 \mathrm{E}+00$ & $3.27 \mathrm{E}-01$ & $\mathrm{cpt}$ \\
\hline 217.8 & $4.97 \mathrm{E}-01$ & $1.38 \mathrm{E}-01$ & $\mathrm{cpt}$ \\
\hline 216.8 & $3.32 \mathrm{E}-01$ & $9.22 \mathrm{E}-02$ & $\mathrm{cpt}$ \\
\hline 215.8 & $2.14 \mathrm{E}+00$ & $5.95 \mathrm{E}-01$ & $\mathrm{cpt}$ \\
\hline 214.8 & $2.16 \mathrm{E}-01$ & $5.99 \mathrm{E}-02$ & $\mathrm{cpt}$ \\
\hline 213.8 & $1.02 \mathrm{E}-03$ & $2.80 \mathrm{E}-04$ & $\mathrm{cpt}$ \\
\hline 212.8 & $4.33 \mathrm{E}-01$ & $1.20 \mathrm{E}-01$ & $\mathrm{cpt}$ \\
\hline 211.8 & $1.66 \mathrm{E}+01$ & $4.61 \mathrm{E}+00$ & $\mathrm{cpt}$ \\
\hline 210.8 & $1.66 \mathrm{E}+01$ & $4.61 \mathrm{E}+00$ & $\mathrm{cpt}$ \\
\hline 209.8 & $1.69 \mathrm{E}+01$ & $4.68 \mathrm{E}+00$ & $\mathrm{cpt}$ \\
\hline 208.8 & $1.90 \mathrm{E}+01$ & $5.28 \mathrm{E}+00$ & $\mathrm{cpt}$ \\
\hline 207.8 & $2.00 \mathrm{E}+01$ & $5.57 \mathrm{E}+00$ & $\mathrm{cpt}$ \\
\hline 206.8 & $1.98 \mathrm{E}+01$ & $5.50 \mathrm{E}+00$ & $\mathrm{cpt}$ \\
\hline 205.8 & $2.14 \mathrm{E}+01$ & $5.94 \mathrm{E}+00$ & $\mathrm{cpt}$ \\
\hline 204.8 & $2.43 \mathrm{E}+01$ & $6.76 \mathrm{E}+00$ & $\mathrm{cpt}$ \\
\hline 203.8 & $2.65 \mathrm{E}+01$ & $7.37 \mathrm{E}+00$ & $\mathrm{cpt}$ \\
\hline 202.8 & $3.02 \mathrm{E}+01$ & $8.39 \mathrm{E}+00$ & $\mathrm{cpt}$ \\
\hline 201.8 & $3.58 \mathrm{E}+01$ & $9.94 \mathrm{E}+00$ & $\mathrm{cpt}$ \\
\hline 200.8 & $3.45 \mathrm{E}+01$ & $9.57 \mathrm{E}+00$ & $\mathrm{cpt}$ \\
\hline 199.8 & $2.97 \mathrm{E}-01$ & $8.26 \mathrm{E}-02$ & $\mathrm{cpt}$ \\
\hline 198.8 & $6.88 \mathrm{E}-02$ & $1.91 \mathrm{E}-02$ & $\mathrm{cpt}$ \\
\hline 197.8 & $2.63 \mathrm{E}+01$ & $7.30 \mathrm{E}+00$ & $\mathrm{cpt}$ \\
\hline 196.8 & $2.45 \mathrm{E}+01$ & $6.81 \mathrm{E}+00$ & $\mathrm{cpt}$ \\
\hline 194.8 & $9.51 \mathrm{E}+01$ & $2.64 \mathrm{E}+01$ & $\mathrm{cpt}$ \\
\hline $8.49 \mathrm{E}+01$ & $2.36 \mathrm{E}+01$ & $\mathrm{cpt}$ \\
\hline & & & \\
\hline
\end{tabular}


Conductivity Data at RPT1

\begin{tabular}{|c|c|c|c|}
\hline $\begin{array}{c}\text { Elevation } \\
\text { (ft) }\end{array}$ & $\begin{array}{c}\text { Kh } \\
\text { (ft/day) }\end{array}$ & $\begin{array}{c}\text { Kv } \\
\text { (ft/day) }\end{array}$ & $\begin{array}{c}\text { Data } \\
\text { Source }\end{array}$ \\
\hline 193.8 & $3.17 \mathrm{E}+01$ & $8.80 \mathrm{E}+00$ & $\mathrm{cpt}$ \\
\hline 192.8 & $1.81 \mathrm{E}+01$ & $5.04 \mathrm{E}+00$ & $\mathrm{cpt}$ \\
\hline 191.8 & $1.84 \mathrm{E}+01$ & $5.12 \mathrm{E}+00$ & $\mathrm{cpt}$ \\
\hline 190.8 & $2.15 \mathrm{E}+01$ & $5.96 \mathrm{E}+00$ & $\mathrm{cpt}$ \\
\hline 189.8 & $1.83 \mathrm{E}+01$ & $5.08 \mathrm{E}+00$ & $\mathrm{cpt}$ \\
\hline 188.8 & $2.64 \mathrm{E}+01$ & $7.34 \mathrm{E}+00$ & $\mathrm{cpt}$ \\
\hline 187.8 & $1.59 \mathrm{E}+01$ & $4.41 \mathrm{E}+00$ & $\mathrm{cpt}$ \\
\hline 186.8 & $1.27 \mathrm{E}+01$ & $3.53 \mathrm{E}+00$ & $\mathrm{cpt}$ \\
\hline 185.8 & $1.01 \mathrm{E}+01$ & $2.81 \mathrm{E}+00$ & $\mathrm{cpt}$ \\
\hline 184.8 & $9.75 \mathrm{E}+00$ & $2.71 \mathrm{E}+00$ & $\mathrm{cpt}$ \\
\hline 183.8 & $3.88 \mathrm{E}-02$ & $1.08 \mathrm{E}-02$ & $\mathrm{cpt}$ \\
\hline 182.8 & $1.15 \mathrm{E}+01$ & $3.20 \mathrm{E}+00$ & $\mathrm{cpt}$ \\
\hline 181.8 & $1.19 \mathrm{E}+01$ & $3.29 \mathrm{E}+00$ & $\mathrm{cpt}$ \\
\hline 180.8 & $1.18 \mathrm{E}+01$ & $3.28 \mathrm{E}+00$ & $\mathrm{cpt}$ \\
\hline 179.8 & $7.03 \mathrm{E}+00$ & $1.95 \mathrm{E}+00$ & $\mathrm{cpt}$ \\
\hline 178.8 & $1.30 \mathrm{E}+00$ & $3.62 \mathrm{E}-01$ & $\mathrm{cpt}$ \\
\hline 177.8 & $4.02 \mathrm{E}-03$ & $1.12 \mathrm{E}-03$ & $\mathrm{cpt}$ \\
\hline 176.8 & $4.01 \mathrm{E}-03$ & $1.11 \mathrm{E}-03$ & $\mathrm{cpt}$ \\
\hline 175.8 & $8.44 \mathrm{E}-02$ & $2.35 \mathrm{E}-02$ & $\mathrm{cpt}$ \\
\hline 174.8 & $3.96 \mathrm{E}-03$ & $1.10 \mathrm{E}-03$ & $\mathrm{cpt}$ \\
\hline 173.8 & $3.01 \mathrm{E}-03$ & $8.40 \mathrm{E}-04$ & $\mathrm{cpt}$ \\
\hline 172.8 & $3.17 \mathrm{E}-03$ & $8.80 \mathrm{E}-04$ & $\mathrm{cpt}$ \\
\hline 171.8 & $1.91 \mathrm{E}-03$ & $5.30 \mathrm{E}-04$ & $\mathrm{cpt}$ \\
\hline 170.8 & $1.25 \mathrm{E}-03$ & $3.50 \mathrm{E}-04$ & $\mathrm{cpt}$ \\
\hline 169.8 & $8.90 \mathrm{E}-04$ & $2.50 \mathrm{E}-04$ & $\mathrm{cpt}$ \\
\hline 168.8 & $1.96 \mathrm{E}-03$ & $5.40 \mathrm{E}-04$ & $\mathrm{cpt}$ \\
\hline 167.8 & $5.29 \mathrm{E}+00$ & $1.47 \mathrm{E}+00$ & $\mathrm{cpt}$ \\
\hline 166.8 & $4.72 \mathrm{E}+01$ & $1.31 \mathrm{E}+01$ & $\mathrm{cpt}$ \\
\hline & & & \\
\hline
\end{tabular}


Conductivity Data at RPT2

\begin{tabular}{|c|c|c|c|}
\hline $\begin{array}{l}\text { Elevation } \\
\text { (ft) }\end{array}$ & $\begin{array}{c}\text { Kh } \\
\text { (ft/day) }\end{array}$ & $\begin{array}{c}\text { Kv } \\
\text { (ft/day) }\end{array}$ & $\begin{array}{c}\text { Data } \\
\text { Source }\end{array}$ \\
\hline 283.8 & $3.56 E+00$ & $9.89 E-01$ & cpt \\
\hline 282.8 & $3.32 \mathrm{E}+00$ & $9.23 E-01$ & cpt \\
\hline 281.8 & $2.25 \mathrm{E}-01$ & $6.24 E-02$ & cpt \\
\hline 280.8 & $1.92 \mathrm{E}-01$ & $5.35 E-02$ & $\mathrm{cpt}$ \\
\hline 279.8 & $3.48 E+00$ & $9.66 \mathrm{E}-01$ & cpt \\
\hline 278.8 & $3.48 E+00$ & $9.68 \mathrm{E}-01$ & $\mathrm{cpt}$ \\
\hline 277.8 & $4.93 E+00$ & $1.37 E+00$ & $\mathrm{cpt}$ \\
\hline 276.8 & $4.73 E+00$ & $1.31 E+00$ & cpt \\
\hline 275.8 & $5.43 E+00$ & $1.51 \mathrm{E}+00$ & cpt \\
\hline 274.8 & $5.43 E+00$ & $1.51 E+00$ & cpt \\
\hline 273.8 & $6.27 \mathrm{E}+00$ & $1.74 \mathrm{E}+00$ & $\mathrm{cpt}$ \\
\hline 272.8 & $5.69 \mathrm{E}+00$ & $1.58 E+00$ & cpt \\
\hline 271.8 & $7.71 E+00$ & $2.14 \mathrm{E}+00$ & $\mathrm{cpt}$ \\
\hline 270.8 & $6.22 \mathrm{E}+00$ & $1.73 \mathrm{E}+00$ & cpt \\
\hline 269.8 & $4.54 \mathrm{E}+00$ & $1.26 \mathrm{E}+00$ & cpt \\
\hline 268.8 & $6.52 E+00$ & $1.81 E+00$ & $\mathrm{cpt}$ \\
\hline 267.8 & $7.15 \mathrm{E}+00$ & $1.99 E+00$ & cpt \\
\hline 266.8 & $1.66 \mathrm{E}+01$ & $4.60 E+00$ & $\mathrm{cpt}$ \\
\hline 265.8 & $4.76 \mathrm{E}+00$ & $1.32 E+00$ & cpt \\
\hline 264.8 & $8.97 \mathrm{E}+00$ & $2.49 E+00$ & cpt \\
\hline 263.8 & $4.94 E+00$ & $1.37 \mathrm{E}+00$ & cpt \\
\hline 262.8 & $4.15 \mathrm{E}+00$ & $1.15 E+00$ & cpt \\
\hline 261.8 & $6.05 \mathrm{E}+00$ & $1.68 \mathrm{E}+00$ & cpt \\
\hline 260.8 & $1.65 \mathrm{E}+01$ & $4.59 E+00$ & $\mathrm{cpt}$ \\
\hline 259.8 & $3.70 \mathrm{E}+01$ & $1.03 E+01$ & cpt \\
\hline 258.8 & $5.18 \mathrm{E}+01$ & $1.44 \mathrm{E}+01$ & $\mathrm{cpt}$ \\
\hline 257.8 & $2.96 \mathrm{E}+01$ & $8.23 E+00$ & cpt \\
\hline 256.8 & $3.12 \mathrm{E}+01$ & $8.66 \mathrm{E}+00$ & cpt \\
\hline 255.8 & $2.87 \mathrm{E}+01$ & $7.98 \mathrm{E}+00$ & cpt \\
\hline 254.8 & $2.33 E+01$ & $6.48 \mathrm{E}+00$ & $\mathrm{cpt}$ \\
\hline 253.8 & $2.76 \mathrm{E}+01$ & $7.66 \mathrm{E}+00$ & cpt \\
\hline 252.8 & $2.40 \mathrm{E}+01$ & $6.68 \mathrm{E}+00$ & $\mathrm{cpt}$ \\
\hline 251.8 & $2.10 \mathrm{E}+01$ & $5.84 E+00$ & cpt \\
\hline 250.8 & $1.69 \mathrm{E}+01$ & $4.70 E+00$ & cpt \\
\hline 249.8 & $3.08 E+01$ & $8.55 E+00$ & cpt \\
\hline 248.8 & $3.03 E+01$ & $8.42 E+00$ & $\mathrm{cpt}$ \\
\hline 247.8 & $2.86 \mathrm{E}+01$ & $7.93 \mathrm{E}+00$ & cpt \\
\hline 246.8 & $2.50 \mathrm{E}+01$ & $6.94 \mathrm{E}+00$ & $\mathrm{cpt}$ \\
\hline 245.8 & $1.31 E+01$ & $3.64 \mathrm{E}+00$ & cpt \\
\hline 244.8 & $5.86 \mathrm{E}+00$ & $1.63 \mathrm{E}+00$ & $c p t$ \\
\hline 243.8 & $7.42 \mathrm{E}+00$ & $2.06 \mathrm{E}+00$ & $\mathrm{cpt}$ \\
\hline 242.8 & $8.06 E+00$ & $2.24 E+00$ & $\mathrm{cpt}$ \\
\hline 241.8 & $7.49 \mathrm{E}+00$ & $2.08 E+00$ & cpt \\
\hline 240.8 & $7.01 \mathrm{E}+00$ & $1.95 \mathrm{E}+00$ & $\mathrm{cpt}$ \\
\hline 239.8 & $2.03 E-02$ & $5.64 \mathrm{E}-03$ & $\mathrm{cpt}$ \\
\hline 238.8 & $3.02 E+00$ & $8.40 \mathrm{E}-01$ & cpt \\
\hline 237.8 & $2.34 \mathrm{E}-01$ & 6.51E-02 & cpt \\
\hline 236.8 & $1.54 \mathrm{E}+00$ & 4.27E-01 & $\mathrm{cpt}$ \\
\hline
\end{tabular}


Conductivity Data at RPT2

\begin{tabular}{|c|c|c|c|}
\hline $\begin{array}{c}\text { Elevation } \\
\text { (ft) }\end{array}$ & $\begin{array}{c}\text { Kh } \\
\text { (ft/day) }\end{array}$ & $\begin{array}{c}\mathrm{Kv} \\
\text { (ft/day) }\end{array}$ & $\begin{array}{c}\text { Data } \\
\text { Source }\end{array}$ \\
\hline 235.8 & $7.19 E+00$ & $2.00 E+00$ & cpt \\
\hline 234.8 & $8.93 E+00$ & $2.48 E+00$ & $\mathrm{cpt}$ \\
\hline 233.8 & $6.97 E+00$ & $1.94 \mathrm{E}+00$ & $\mathrm{cpt}$ \\
\hline 232.8 & $2.28 E+00$ & $6.34 \mathrm{E}-01$ & $\mathrm{cpt}$ \\
\hline 231.8 & $8.89 E+00$ & $2.47 E+00$ & $\mathrm{cpt}$ \\
\hline 230.8 & $3.54 E+00$ & $9.84 \mathrm{E}-01$ & $\mathrm{cpt}$ \\
\hline 229.8 & $1.91 \mathrm{E}-01$ & $5.30 E-02$ & $\mathrm{cpt}$ \\
\hline 228.8 & $8.90 \mathrm{E}-04$ & $2.50 \mathrm{E}-04$ & cpt \\
\hline 227.8 & $1.94 \mathrm{E}-03$ & $5.40 E-04$ & $\mathrm{cpt}$ \\
\hline 226.8 & $1.27 \mathrm{E}-03$ & $3.50 E-04$ & cpt \\
\hline 225.8 & $2.15 E-02$ & $5.97 E-03$ & $\mathrm{cpt}$ \\
\hline 224.8 & $4.98 \mathrm{E}-03$ & $1.38 E-03$ & cpt \\
\hline 223.8 & 9.61E-01 & $2.67 \mathrm{E}-01$ & $\mathrm{cpt}$ \\
\hline 222.8 & $8.80 \mathrm{E}-04$ & $2.40 E-04$ & $\mathrm{cpt}$ \\
\hline 221.8 & $5.44 E-03$ & $1.51 \mathrm{E}-03$ & $\mathrm{cpt}$ \\
\hline 220.8 & $1.68 \mathrm{E}-01$ & $4.68 E-02$ & $\mathrm{cpt}$ \\
\hline 219.8 & $5.98 \mathrm{E}-02$ & $1.66 \mathrm{E}-02$ & cpt \\
\hline 218.8 & $1.14 \mathrm{E}+01$ & $3.18 E+00$ & $\mathrm{cpt}$ \\
\hline 217.8 & $1.52 \mathrm{E}+01$ & $4.22 E+00$ & $\mathrm{cpt}$ \\
\hline 216.8 & $1.59 \mathrm{E}+01$ & $4.42 E+00$ & $\mathrm{cpt}$ \\
\hline 215.8 & $1.84 \mathrm{E}+01$ & $5.10 \mathrm{E}+00$ & $\mathrm{cpt}$ \\
\hline 214.8 & $4.36 \mathrm{E}+00$ & $1.21 \mathrm{E}+00$ & $\mathrm{cpt}$ \\
\hline 213.8 & $1.59 \mathrm{E}+01$ & $4.42 \mathrm{E}+00$ & $\mathrm{cpt}$ \\
\hline 212.8 & $1.26 \mathrm{E}+01$ & $3.49 \mathrm{E}+00$ & cpt \\
\hline 211.8 & $7.19 E+00$ & $2.00 E+00$ & cpt \\
\hline 210.8 & $1.30 \mathrm{E}+01$ & $3.61 E+00$ & cpt \\
\hline 209.8 & $8.95 E+00$ & $2.49 E+00$ & cpt \\
\hline 208.8 & $1.87 \mathrm{E}+01$ & $5.20 \mathrm{E}+00$ & cpt \\
\hline 207.8 & $1.73 \mathrm{E}+01$ & $4.79 E+00$ & cpt \\
\hline 206.8 & $4.18 E-03$ & 1.16E-03 & $\mathrm{cpt}$ \\
\hline 205.8 & $3.10 \mathrm{E}-03$ & $8.60 E-04$ & cpt \\
\hline 204.8 & $9.84 \mathrm{E}+00$ & $2.73 \mathrm{E}+00$ & $\mathrm{cpt}$ \\
\hline 203.8 & $4.48 E+01$ & $1.24 E+01$ & cpt \\
\hline 202.8 & $6.08 \mathrm{E}+01$ & $1.69 \mathrm{E}+01$ & cpt \\
\hline 201.8 & $5.14 E+01$ & $1.43 E+01$ & cpt \\
\hline 200.8 & $3.97 \mathrm{E}+01$ & 1.10E+01 & cpt \\
\hline 199.8 & $3.16 \mathrm{E}+01$ & $8.79 E+00$ & $\mathrm{cpt}$ \\
\hline 198.8 & $5.52 E+01$ & $1.53 E+01$ & cpt \\
\hline 197.8 & $6.86 \mathrm{E}+01$ & $1.91 E+01$ & cpt \\
\hline 196.8 & $6.19 \mathrm{E}+01$ & $1.72 E+01$ & cpt \\
\hline 195.8 & $5.00 \mathrm{E}+01$ & $1.39 \mathrm{E}+01$ & cpt \\
\hline 194.8 & $1.73 E+01$ & $4.82 E+00$ & cpt \\
\hline 193.8 & $1.80 \mathrm{E}+01$ & $4.99 E+00$ & $\mathrm{cpt}$ \\
\hline 192.8 & $1.19 \mathrm{E}-02$ & $3.32 E-03$ & cpt \\
\hline 191.8 & $2.83 E-03$ & $7.90 \mathrm{E}-04$ & $\mathrm{cpt}$ \\
\hline 190.8 & $2.24 E-03$ & $6.20 \mathrm{E}-04$ & $\mathrm{cpt}$ \\
\hline 189.8 & $2.50 E-03$ & $7.00 \mathrm{E}-04$ & $\mathrm{cpt}$ \\
\hline 188.8 & $7.45 E-03$ & $2.07 E-03$ & cpt \\
\hline
\end{tabular}


Conductivity Data at RPT2

\begin{tabular}{|c|c|c|c|}
\hline $\begin{array}{c}\text { Elevation } \\
\text { (ft) }\end{array}$ & $\begin{array}{c}\text { Kh } \\
\text { (ft/day) }\end{array}$ & $\begin{array}{c}\text { Kv } \\
\text { (ft/day) }\end{array}$ & $\begin{array}{c}\text { Data } \\
\text { Source }\end{array}$ \\
\hline 187.8 & $2.69 \mathrm{E}-03$ & $7.50 \mathrm{E}-04$ & $\mathrm{cpt}$ \\
\hline 186.8 & $2.82 \mathrm{E}-03$ & $7.80 \mathrm{E}-04$ & $\mathrm{cpt}$ \\
\hline 185.8 & $6.20 \mathrm{E}-03$ & $1.72 \mathrm{E}-03$ & $\mathrm{cpt}$ \\
\hline 184.8 & $2.31 \mathrm{E}-03$ & $6.40 \mathrm{E}-04$ & $\mathrm{cpt}$ \\
\hline 183.8 & $2.46 \mathrm{E}-03$ & $6.80 \mathrm{E}-04$ & $\mathrm{cpt}$ \\
\hline 182.8 & $1.04 \mathrm{E}-03$ & $2.90 \mathrm{E}-04$ & $\mathrm{cpt}$ \\
\hline 181.8 & $1.06 \mathrm{E}-03$ & $3.00 \mathrm{E}-04$ & $\mathrm{cpt}$ \\
\hline 180.8 & $2.94 \mathrm{E}-03$ & $8.20 \mathrm{E}-04$ & $\mathrm{cpt}$ \\
\hline 179.8 & $2.78 \mathrm{E}-03$ & $7.70 \mathrm{E}-04$ & $\mathrm{cpt}$ \\
\hline 178.8 & $2.35 \mathrm{E}-03$ & $6.50 \mathrm{E}-04$ & $\mathrm{cpt}$ \\
\hline 177.8 & $3.07 \mathrm{E}-03$ & $8.50 \mathrm{E}-04$ & $\mathrm{cpt}$ \\
\hline 176.8 & $3.38 \mathrm{E}-03$ & $9.40 \mathrm{E}-04$ & $\mathrm{cpt}$ \\
\hline 175.8 & $1.11 \mathrm{E}-02$ & $3.09 \mathrm{E}-03$ & $\mathrm{cpt}$ \\
\hline 174.8 & $4.22 \mathrm{E}-03$ & $1.17 \mathrm{E}-03$ & $\mathrm{cpt}$ \\
\hline 173.8 & $2.28 \mathrm{E}-03$ & $6.30 \mathrm{E}-04$ & $\mathrm{cpt}$ \\
\hline 172.8 & $1.17 \mathrm{E}-03$ & $3.20 \mathrm{E}-04$ & $\mathrm{cpt}$ \\
\hline 171.8 & $1.22 \mathrm{E}-03$ & $3.40 \mathrm{E}-04$ & $\mathrm{cpt}$ \\
\hline 170.8 & $1.19 \mathrm{E}-03$ & $3.30 \mathrm{E}-04$ & $\mathrm{cpt}$ \\
\hline 169.8 & $1.20 \mathrm{E}-03$ & $3.30 \mathrm{E}-04$ & $\mathrm{cpt}$ \\
\hline 168.8 & $1.98 \mathrm{E}-03$ & $5.50 \mathrm{E}-04$ & $\mathrm{cpt}$ \\
\hline 167.8 & $2.82 \mathrm{E}-03$ & $7.80 \mathrm{E}-04$ & $\mathrm{cpt}$ \\
\hline 166.8 & $1.01 \mathrm{E}-02$ & $2.80 \mathrm{E}-03$ & $\mathrm{cpt}$ \\
\hline 165.8 & $1.25 \mathrm{E}-03$ & $3.50 \mathrm{E}-04$ & $\mathrm{cpt}$ \\
\hline 164.8 & $1.20 \mathrm{E}-02$ & $3.34 \mathrm{E}-03$ & $\mathrm{cpt}$ \\
\hline 163.8 & $1.42 \mathrm{E}+01$ & $3.94 \mathrm{E}+00$ & $\mathrm{cpt}$ \\
\hline 162.8 & $3.62 \mathrm{E}+01$ & $1.01 \mathrm{E}+01$ & $\mathrm{cpt}$ \\
\hline 161.8 & $6.01 \mathrm{E}+01$ & $1.67 \mathrm{E}+01$ & $\mathrm{cpt}$ \\
\hline 160.8 & $1.00 \mathrm{E}+02$ & $2.78 \mathrm{E}+01$ & $\mathrm{cpt}$ \\
\hline 159.8 & $1.00 \mathrm{E}+02$ & $2.78 \mathrm{E}+01$ & $\mathrm{cpt}$ \\
\hline 158.8 & $9.01 \mathrm{E}+01$ & $2.50 \mathrm{E}+01$ & $\mathrm{cpt}$ \\
\hline 157.8 & $1.00 \mathrm{E}+02$ & $2.78 \mathrm{E}+01$ & $\mathrm{cpt}$ \\
\hline 156.8 & $1.00 \mathrm{E}+02$ & $2.78 \mathrm{E}+01$ & $\mathrm{cpt}$ \\
\hline 155.8 & $1.00 \mathrm{E}+02$ & $2.78 \mathrm{E}+01$ & $\mathrm{cpt}$ \\
\hline 154.8 & $4.38 \mathrm{E}+01$ & $1.22 \mathrm{E}+01$ & $\mathrm{cpt}$ \\
\hline 153.8 & $6.50 \mathrm{E}+01$ & $1.81 \mathrm{E}+01$ & $\mathrm{cpt}$ \\
\hline 152.8 & $5.13 \mathrm{E}+01$ & $1.43 \mathrm{E}+01$ & $\mathrm{cpt}$ \\
\hline 151.8 & $3.99 \mathrm{E}+01$ & $1.11 \mathrm{E}+01$ & $\mathrm{cpt}$ \\
\hline 150.8 & $1.10 \mathrm{E}+01$ & $3.05 \mathrm{E}+00$ & $\mathrm{cpt}$ \\
\hline 149.8 & $1.59 \mathrm{E}+01$ & $4.41 \mathrm{E}+00$ & $\mathrm{cpt}$ \\
\hline 148.8 & $3.23 \mathrm{E}+01$ & $8.96 \mathrm{E}+00$ & $\mathrm{cpt}$ \\
\hline 147.8 & $3.21 \mathrm{E}+01$ & $8.90 \mathrm{E}+00$ & $\mathrm{cpt}$ \\
\hline 146.8 & $1.00 \mathrm{E}+02$ & $2.78 \mathrm{E}+01$ & $\mathrm{cpt}$ \\
\hline 145.8 & $2.76 \mathrm{E}+01$ & $7.66 \mathrm{E}+00$ & $\mathrm{cpt}$ \\
\hline 144.8 & $1.00 \mathrm{E}+02$ & $2.78 \mathrm{E}+01$ & $\mathrm{cpt}$ \\
\hline & & & \\
\hline
\end{tabular}




\section{Conductivity Data at RPT3}

\begin{tabular}{|c|c|c|c|}
\hline $\begin{array}{l}\text { Elevation } \\
\text { (ft) }\end{array}$ & $\begin{array}{c}\text { Kh } \\
\text { (ftdday) }\end{array}$ & $\begin{array}{c}\text { Kv } \\
\text { (ft/day) }\end{array}$ & $\begin{array}{c}\text { Data } \\
\text { Source }\end{array}$ \\
\hline 297.8 & $1.22 \mathrm{E}-02$ & $3.38 E-03$ & $\mathrm{cpt}$ \\
\hline 296.8 & $2.70 \mathrm{E}-04$ & $7.00 \mathrm{E}-05$ & cpt \\
\hline 295.8 & $4.38 \mathrm{E}+00$ & $1.22 \mathrm{E}+00$ & $\mathrm{cpt}$ \\
\hline 294.8 & $3.94 \mathrm{E}+00$ & $1.10 \mathrm{E}+00$ & $\mathrm{cpt}$ \\
\hline 293.8 & $3.91 \mathrm{E}+00$ & $1.09 \mathrm{E}+00$ & $\mathrm{cpt}$ \\
\hline 292.8 & $3.93 \mathrm{E}+00$ & $1.09 \mathrm{E}+00$ & $\mathrm{cpt}$ \\
\hline 291.8 & $5.39 \mathrm{E}+00$ & $1.50 \mathrm{E}+00$ & cpt \\
\hline 290.8 & $3.07 \mathrm{E}+00$ & $8.53 E-01$ & cpt \\
\hline 289.8 & $4.05 E+00$ & $1.13 \mathrm{E}+00$ & $\mathrm{cpt}$ \\
\hline 288.8 & $3.73 \mathrm{E}+00$ & $1.04 \mathrm{E}+00$ & cpt \\
\hline 287.8 & $5.39 \mathrm{E}+00$ & $1.50 \mathrm{E}+00$ & cpt \\
\hline 286.8 & $2.05 E-03$ & $5.70 \mathrm{E}-04$ & cpt \\
\hline 285.8 & $7.47 \mathrm{E}+00$ & $2.08 \mathrm{E}+00$ & cpt \\
\hline 284.8 & $1.51 \mathrm{E}+01$ & $4.20 E+00$ & $\mathrm{cpt}$ \\
\hline 283.8 & $3.91 \mathrm{E}+00$ & $1.09 \mathrm{E}+00$ & $\mathrm{cpt}$ \\
\hline 282.8 & $1.48 \mathrm{E}-03$ & $4.10 \mathrm{E}-04$ & $\mathrm{cpt}$ \\
\hline 281.8 & 1.11E-03 & $3.10 \mathrm{E}-04$ & $\mathrm{cpt}$ \\
\hline 280.8 & $1.57 E+01$ & $4.37 E+00$ & cpt \\
\hline 279.8 & $1.21 \mathrm{E}-03$ & $3.30 \mathrm{E}-04$ & $\mathrm{cpt}$ \\
\hline 278.8 & $8.80 \mathrm{E}-04$ & $2.50 E-04$ & cpt \\
\hline 277.8 & $9.50 \mathrm{E}-04$ & $2.60 E-04$ & $\mathrm{cpt}$ \\
\hline 276.8 & $7.68 \mathrm{E}+00$ & $2.13 \mathrm{E}+00$ & $\mathrm{cpt}$ \\
\hline 275.8 & $4.06 \mathrm{E}-02$ & $1.13 \mathrm{E}-02$ & $\mathrm{cpt}$ \\
\hline 274.8 & $6.63 \mathrm{E}+00$ & $1.84 \mathrm{E}+00$ & cpt \\
\hline 273.8 & $7.74 \mathrm{E}+00$ & $2.15 \mathrm{E}+00$ & $\mathrm{cpt}$ \\
\hline 272.8 & $1.40 \mathrm{E}+00$ & $3.90 \mathrm{E}-01$ & cpt \\
\hline 271.8 & $1.92 \mathrm{E}-02$ & $5.34 \mathrm{E}-03$ & $\mathrm{cpt}$ \\
\hline 270.8 & $4.24 \mathrm{E}+00$ & $1.18 \mathrm{E}+00$ & cpt \\
\hline 269.8 & $1.08 \mathrm{E}-03$ & $3.00 \mathrm{E}-04$ & cpt \\
\hline 268.8 & $6.80 \mathrm{E}-04$ & 1.90E-04 & cpt \\
\hline 267.8 & $8.78 \mathrm{E}-03$ & $2.44 \mathrm{E}-03$ & $\mathrm{cpt}$ \\
\hline 266.8 & $6.10 \mathrm{E}-04$ & $1.70 E-04$ & $\mathrm{cpt}$ \\
\hline 265.8 & $5.70 \mathrm{E}-04$ & $1.60 \mathrm{E}-04$ & $\mathrm{cpt}$ \\
\hline 264.8 & $7.10 \mathrm{E}-04$ & $2.00 E-04$ & $c p t$ \\
\hline 263.8 & $6.60 \mathrm{E}-04$ & $1.80 E-04$ & $\mathrm{cpt}$ \\
\hline 262.8 & $6.20 \mathrm{E}-04$ & $1.70 \mathrm{E}-04$ & $\mathrm{cpt}$ \\
\hline 261.8 & $1.36 \mathrm{E}-03$ & $3.80 \mathrm{E}-04$ & cpt \\
\hline 260.8 & $6.10 \mathrm{E}-04$ & $1.70 \mathrm{E}-04$ & cpt \\
\hline 259.8 & $4.90 \mathrm{E}-04$ & $1.40 \mathrm{E}-04$ & $\mathrm{cpt}$ \\
\hline 258.8 & $5.40 \mathrm{E}-04$ & $1.50 \mathrm{E}-04$ & $\mathrm{cpt}$ \\
\hline 257.8 & $5.00 \mathrm{E}-04$ & $1.40 \mathrm{E}-04$ & $\mathrm{cpt}$ \\
\hline 256.8 & $4.70 \mathrm{E}-04$ & 1.30E-04 & $\mathrm{cpt}$ \\
\hline 255.8 & $4.60 \mathrm{E}-04$ & $1.30 \mathrm{E}-04$ & cpt \\
\hline 254.8 & $4.40 \mathrm{E}-04$ & $1.20 \mathrm{E}-04$ & $\mathrm{cpt}$ \\
\hline 253.8 & $4.30 \mathrm{E}-04$ & $1.20 \mathrm{E}-04$ & $\mathrm{cpt}$ \\
\hline 252.8 & $4.40 \mathrm{E}-04$ & $1.20 \mathrm{E}-04$ & $\mathrm{cpt}$ \\
\hline 251.8 & $4.10 \mathrm{E}-04$ & $1.10 \mathrm{E}-04$ & $\mathrm{cpt}$ \\
\hline 250.8 & $4.70 \mathrm{E}-03$ & $1.31 \mathrm{E}-03$ & $\mathrm{cpt}$ \\
\hline
\end{tabular}


Conductivity Data at RPT3

\begin{tabular}{|c|c|c|c|}
\hline $\begin{array}{c}\text { Elevation } \\
\text { (ft) }\end{array}$ & $\begin{array}{c}\text { Kh } \\
\text { (ft/day) }\end{array}$ & $\begin{array}{c}\text { Kv } \\
\text { (ft/day) }\end{array}$ & $\begin{array}{c}\text { Data } \\
\text { Source }\end{array}$ \\
\hline 249.8 & $1.07 E-02$ & $2.97 \mathrm{E}-03$ & $\mathrm{cpt}$ \\
\hline 248.8 & $6.93 E-03$ & $1.93 \mathrm{E}-03$ & $\mathrm{cpt}$ \\
\hline 247.8 & $2.52 E-03$ & $7.00 \mathrm{E}-04$ & $\overline{c p t}$ \\
\hline 246.8 & $4.20 \mathrm{E}-04$ & $1.20 \mathrm{E}-04$ & $\mathrm{cpt}$ \\
\hline 245.8 & $4.40 \mathrm{E}-04$ & $1.20 \mathrm{E}-04$ & $\mathrm{cpt}$ \\
\hline 244.8 & $1.92 E+01$ & $5.35 E+00$ & cpt \\
\hline 243.8 & $1.54 \mathrm{E}+01$ & $4.27 E+00$ & cpt \\
\hline 242.8 & $6.49 \mathrm{E}+00$ & $1.80 \mathrm{E}+00$ & cpt \\
\hline 241.8 & $6.14 \mathrm{E}+00$ & $1.71 \mathrm{E}+00$ & cpt \\
\hline 240.8 & $7.80 \mathrm{E}-02$ & $2.17 \mathrm{E}-02$ & $\mathrm{cpt}$ \\
\hline 239.8 & $1.44 \mathrm{E}+01$ & $4.01 \mathrm{E}+00$ & cpt \\
\hline 238.8 & $1.11 \mathrm{E}+01$ & $3.07 E+00$ & $\mathrm{cpt}$ \\
\hline 237.8 & $3.13 \mathrm{E}-01$ & $8.69 \mathrm{E}-02$ & $\mathrm{cpt}$ \\
\hline 236.8 & $1.05 E-01$ & $2.90 \mathrm{E}-02$ & $\mathrm{cpt}$ \\
\hline 235.8 & $8.76 \mathrm{E}+00$ & $2.43 E+00$ & $\mathrm{cpt}$ \\
\hline 234.8 & $2.97 \mathrm{E}+00$ & $8.25 \mathrm{E}-01$ & $\mathrm{cpt}$ \\
\hline 233.8 & $4.44 E+00$ & $1.23 E+00$ & $\mathrm{cpt}$ \\
\hline 232.8 & $1.01 \mathrm{E}+00$ & $2.80 \mathrm{E}-01$ & cpt \\
\hline 231.8 & $8.73 E+00$ & $2.43 E+00$ & $\mathrm{cpt}$ \\
\hline 230.8 & $2.24 \mathrm{E}+01$ & $6.23 E+00$ & $\mathrm{cpt}$ \\
\hline 229.8 & $1.18 \mathrm{E}+01$ & $3.28 \mathrm{E}+00$ & cpt \\
\hline 228.8 & $9.17 E+00$ & $2.55 \mathrm{E}+00$ & $\mathrm{cpt}$ \\
\hline 227.8 & 3.71E-02 & $1.03 E-02$ & $\mathrm{cpt}$ \\
\hline 226.8 & $2.28 \mathrm{E}-01$ & $6.34 \mathrm{E}-02$ & cpt \\
\hline 225.8 & $9.66 \mathrm{E}+00$ & $2.68 E+00$ & $\mathrm{cpt}$ \\
\hline 224.8 & $7.55 \mathrm{E}+00$ & $2.10 E+00$ & $c p t$ \\
\hline 223.8 & $8.12 E+00$ & $2.25 \mathrm{E}+00$ & cpt \\
\hline 222.8 & $6.81 \mathrm{E}+00$ & $1.89 \mathrm{E}+00$ & cpt \\
\hline 221.8 & $1.40 \mathrm{E}+00$ & $3.88 \mathrm{E}-01$ & $\mathrm{cpt}$ \\
\hline 220.8 & $1.36 \mathrm{E}-03$ & $3.80 \mathrm{E}-04$ & $\mathrm{cpt}$ \\
\hline 219.8 & $3.32 \mathrm{E}-01$ & $9.23 \mathrm{E}-02$ & cpt \\
\hline 218.8 & $8.73 E-02$ & $2.43 E-02$ & $\mathrm{cpt}$ \\
\hline 217.8 & $6.70 \mathrm{E}-04$ & $1.90 \mathrm{E}-04$ & $\mathrm{cpt}$ \\
\hline 216.8 & $1.14 \mathrm{E}+01$ & $3.17 E+00$ & $\mathrm{cpt}$ \\
\hline 215.8 & $1.52 \mathrm{E}+00$ & $4.22 \mathrm{E}-01$ & $c p t$ \\
\hline 214.8 & $8.08 \mathrm{E}-03$ & $2.24 \mathrm{E}-03$ & cpt \\
\hline 213.8 & $6.81 \mathrm{E}-01$ & $1.89 \mathrm{E}-01$ & $\mathrm{cpt}$ \\
\hline 212.8 & $6.24 \mathrm{E}-03$ & 1.73E-03 & $\mathrm{cpt}$ \\
\hline 211.8 & $2.54 \mathrm{E}-02$ & $7.07 \mathrm{E}-03$ & $\mathrm{cpt}$ \\
\hline 210.8 & $4.04 \mathrm{E}-01$ & $1.12 \mathrm{E}-01$ & $c p t$ \\
\hline 209.8 & $1.01 \mathrm{E}-01$ & $2.81 E-02$ & $\mathrm{cpt}$ \\
\hline 208.8 & 7.14E-02 & $1.98 \mathrm{E}-02$ & cpt \\
\hline 207.8 & $1.16 \mathrm{E}+00$ & $3.23 E-01$ & $\mathrm{cpt}$ \\
\hline 206.8 & $7.66 \mathrm{E}+00$ & $2.13 E+00$ & $\mathrm{cpt}$ \\
\hline 205.8 & $6.61 E+00$ & $1.84 \mathrm{E}+00$ & $\mathrm{cpt}$ \\
\hline 204.8 & $6.66 \mathrm{E}+00$ & $1.85 \mathrm{E}+00$ & $\mathrm{cpt}$ \\
\hline 203.8 & $6.21 \mathrm{E}-01$ & $1.73 E-01$ & $\mathrm{cpt}$ \\
\hline 202.8 & $7.60 \mathrm{E}-04$ & $2.10 \mathrm{E}-04$ & $\mathrm{cpt}$ \\
\hline
\end{tabular}


Conductivity Data at RPT3

\begin{tabular}{|c|c|c|c|}
\hline $\begin{array}{c}\text { Elevation } \\
\text { (ft) }\end{array}$ & $\begin{array}{c}\text { Kh } \\
\text { (ftday) }\end{array}$ & $\begin{array}{c}\text { Kv } \\
\text { (ft/day) }\end{array}$ & $\begin{array}{c}\text { Data } \\
\text { source }\end{array}$ \\
\hline 201.8 & $1.75 \mathrm{E}+01$ & $4.87 \mathrm{E}+00$ & $\mathrm{cpt}$ \\
\hline 200.8 & $1.76 \mathrm{E}+01$ & $4.89 \mathrm{E}+00$ & $\mathrm{cpt}$ \\
\hline 199.8 & $1.93 \mathrm{E}+01$ & $5.37 \mathrm{E}+00$ & $\mathrm{cpt}$ \\
\hline 198.8 & $1.81 \mathrm{E}+01$ & $5.02 \mathrm{E}+00$ & $\mathrm{cpt}$ \\
\hline 197.8 & $2.14 \mathrm{E}+01$ & $5.94 \mathrm{E}+00$ & $\mathrm{cpt}$ \\
\hline 196.8 & $1.92 \mathrm{E}+01$ & $5.32 \mathrm{E}+00$ & $\mathrm{cpt}$ \\
\hline 195.8 & $1.64 \mathrm{E}+01$ & $4.56 \mathrm{E}+00$ & $\mathrm{cpt}$ \\
\hline 194.8 & $1.96 \mathrm{E}+01$ & $5.46 \mathrm{E}+00$ & $\mathrm{cpt}$ \\
\hline 193.8 & $2.29 \mathrm{E}+01$ & $6.37 \mathrm{E}+00$ & $\mathrm{cpt}$ \\
\hline 192.8 & $2.62 \mathrm{E}+01$ & $7.27 \mathrm{E}+00$ & $\mathrm{cpt}$ \\
\hline 191.8 & $2.75 \mathrm{E}+01$ & $7.63 \mathrm{E}+00$ & $\mathrm{cpt}$ \\
\hline 190.8 & $2.83 \mathrm{E}+01$ & $7.87 \mathrm{E}+00$ & $\mathrm{cpt}$ \\
\hline 189.8 & $1.52 \mathrm{E}-03$ & $4.20 \mathrm{E}-04$ & $\mathrm{cpt}$ \\
\hline 188.8 & $1.26 \mathrm{E}-03$ & $3.50 \mathrm{E}-04$ & $\mathrm{cpt}$ \\
\hline 187.8 & $3.09 \mathrm{E}+01$ & $8.59 \mathrm{E}+00$ & $\mathrm{cpt}$ \\
\hline 186.8 & $6.63 \mathrm{E}+01$ & $1.84 \mathrm{E}+01$ & $\mathrm{cpt}$ \\
\hline 185.8 & $5.18 \mathrm{E}+01$ & $1.44 \mathrm{E}+01$ & $\mathrm{cpt}$ \\
\hline 184.8 & $4.76 \mathrm{E}+01$ & $1.32 \mathrm{E}+01$ & $\mathrm{cpt}$ \\
\hline 183.8 & $4.72 \mathrm{E}+01$ & $1.31 \mathrm{E}+01$ & $\mathrm{cpt}$ \\
\hline 182.8 & $7.29 \mathrm{E}+01$ & $2.02 \mathrm{E}+01$ & $\mathrm{cpt}$ \\
\hline 181.8 & $7.99 \mathrm{E}+01$ & $2.22 \mathrm{E}+01$ & $\mathrm{cpt}$ \\
\hline 180.8 & $8.10 \mathrm{E}+01$ & $2.25 \mathrm{E}+01$ & $\mathrm{cpt}$ \\
\hline 179.8 & $7.52 \mathrm{E}+01$ & $2.09 \mathrm{E}+01$ & $\mathrm{cpt}$ \\
\hline 178.8 & $4.69 \mathrm{E}+01$ & $1.30 \mathrm{E}+01$ & $\mathrm{cpt}$ \\
\hline 177.8 & $4.31 \mathrm{E}+01$ & $1.20 \mathrm{E}+01$ & $\mathrm{cpt}$ \\
\hline 176.8 & $1.30 \mathrm{E}-03$ & $3.60 \mathrm{E}-04$ & $\mathrm{cpt}$ \\
\hline 175.8 & $5.00 \mathrm{E}-04$ & $1.40 \mathrm{E}-04$ & $\mathrm{cpt}$ \\
\hline 174.8 & $3.78 \mathrm{E}+01$ & $1.05 \mathrm{E}+01$ & $\mathrm{cpt}$ \\
\hline 173.8 & $4.62 \mathrm{E}+01$ & $1.28 \mathrm{E}+01$ & $\mathrm{cpt}$ \\
\hline 172.8 & $3.84 \mathrm{E}+01$ & $1.07 \mathrm{E}+01$ & $\mathrm{cpt}$ \\
\hline 171.8 & $5.03 \mathrm{E}+01$ & $1.40 \mathrm{E}+01$ & $\mathrm{cpt}$ \\
\hline 170.8 & $2.71 \mathrm{E}+01$ & $7.53 \mathrm{E}+00$ & $\mathrm{cpt}$ \\
\hline 169.8 & $5.37 \mathrm{E}+01$ & $1.49 \mathrm{E}+01$ & $\mathrm{cpt}$ \\
\hline 168.8 & $7.60 \mathrm{E}+01$ & $2.11 \mathrm{E}+01$ & $\mathrm{cpt}$ \\
\hline 167.8 & $3.05 \mathrm{E}+01$ & $8.48 \mathrm{E}+00$ & $\mathrm{cpt}$ \\
\hline 166.8 & $9.59 \mathrm{E}-03$ & $2.67 \mathrm{E}-03$ & $\mathrm{cpt}$ \\
\hline 165.8 & $1.84 \mathrm{E}-03$ & $5.10 \mathrm{E}-04$ & $\mathrm{cpt}$ \\
\hline 164.8 & $7.62 \mathrm{E}+01$ & $2.12 \mathrm{E}+01$ & $\mathrm{cpt}$ \\
\hline 163.8 & $7.25 \mathrm{E}+01$ & $2.01 \mathrm{E}+01$ & $\mathrm{cpt}$ \\
\hline 162.8 & $6.22 \mathrm{E}+01$ & $1.73 \mathrm{E}+01$ & $\mathrm{cpt}$ \\
\hline 161.8 & $7.04 \mathrm{E}+01$ & $1.96 \mathrm{E}+01$ & $\mathrm{cpt}$ \\
\hline 160.8 & $7.48 \mathrm{E}+01$ & $2.08 \mathrm{E}+01$ & $\mathrm{cpt}$ \\
\hline 159.8 & $5.61 \mathrm{E}+01$ & $1.56 \mathrm{E}+01$ & $\mathrm{cpt}$ \\
\hline 158.8 & $3.30 \mathrm{E}-03$ & $9.20 \mathrm{E}-04$ & $\mathrm{cpt}$ \\
\hline 157.8 & $1.28 \mathrm{E}-02$ & $3.55 \mathrm{E}-03$ & $\mathrm{cpt}$ \\
\hline 155.8 & $1.74 \mathrm{E}-03$ & $4.80 \mathrm{E}-04$ & $\mathrm{cpt}$ \\
\hline 154.8 & $6.47 \mathrm{E}+01$ & $1.80 \mathrm{E}+01$ & $\mathrm{cpt}$ \\
\hline & $9.57 \mathrm{E}+01$ & $2.66 \mathrm{E}+01$ & $\mathrm{cpt}$ \\
\hline & & \\
\hline
\end{tabular}


Conductivity Data at RPT3

\begin{tabular}{|c|c|c|c|}
\hline $\begin{array}{c}\text { Elevation } \\
\text { (ft) }\end{array}$ & $\begin{array}{c}\text { Kh } \\
\text { (ft/day) }\end{array}$ & $\begin{array}{c}\text { Kv } \\
\text { (ft/day) }\end{array}$ & $\begin{array}{c}\text { Data } \\
\text { Source }\end{array}$ \\
\hline 153.8 & $1.00 \mathrm{E}+02$ & $2.78 \mathrm{E}+01$ & $\mathrm{cpt}$ \\
\hline 152.8 & $5.32 \mathrm{E}+01$ & $1.48 \mathrm{E}+01$ & $\mathrm{cpt}$ \\
\hline 151.8 & $1.00 \mathrm{E}+02$ & $2.78 \mathrm{E}+01$ & $\mathrm{cpt}$ \\
\hline 150.8 & $7.30 \mathrm{E}+01$ & $2.03 \mathrm{E}+01$ & $\mathrm{cpt}$ \\
\hline 149.8 & $1.00 \mathrm{E}+02$ & $2.78 \mathrm{E}+01$ & $\mathrm{cpt}$ \\
\hline 148.8 & $1.00 \mathrm{E}+02$ & $2.78 \mathrm{E}+01$ & $\mathrm{cpt}$ \\
\hline 147.8 & $6.29 \mathrm{E}+01$ & $1.75 \mathrm{E}+01$ & $\mathrm{cpt}$ \\
\hline 146.8 & $1.00 \mathrm{E}+02$ & $2.78 \mathrm{E}+01$ & $\mathrm{cpt}$ \\
\hline 145.8 & $6.17 \mathrm{E}+01$ & $1.71 \mathrm{E}+01$ & $\mathrm{cpt}$ \\
\hline 144.8 & $1.76 \mathrm{E}-01$ & $4.89 \mathrm{E}-02$ & $\mathrm{cpt}$ \\
\hline 143.8 & $9.98 \mathrm{E}+01$ & $2.77 \mathrm{E}+01$ & $\mathrm{cpt}$ \\
\hline 142.8 & $1.00 \mathrm{E}+02$ & $2.78 \mathrm{E}+01$ & $\mathrm{cpt}$ \\
\hline 141.8 & $1.00 \mathrm{E}+02$ & $2.78 \mathrm{E}+01$ & $\mathrm{cpt}$ \\
\hline 140.8 & $4.23 \mathrm{E}+01$ & $1.18 \mathrm{E}+01$ & $\mathrm{cpt}$ \\
\hline 139.8 & $7.59 \mathrm{E}+01$ & $2.11 \mathrm{E}+01$ & $\mathrm{cpt}$ \\
\hline 138.8 & $6.77 \mathrm{E}+01$ & $1.88 \mathrm{E}+01$ & $\mathrm{cpt}$ \\
\hline 137.8 & $4.54 \mathrm{E}+01$ & $1.26 \mathrm{E}+01$ & $\mathrm{cpt}$ \\
\hline 136.8 & $2.64 \mathrm{E}+00$ & $7.33 \mathrm{E}-01$ & $\mathrm{cpt}$ \\
\hline 135.8 & $1.00 \mathrm{E}+02$ & $2.78 \mathrm{E}+01$ & $\mathrm{cpt}$ \\
\hline
\end{tabular}


Conductivity Data at RPT5

\begin{tabular}{|c|c|c|c|}
\hline $\begin{array}{c}\text { Elevation } \\
\text { (ft) }\end{array}$ & $\begin{array}{c}\text { Kh } \\
\text { (ft/day) }\end{array}$ & $\begin{array}{c}\text { Kv } \\
\text { (ft/day) }\end{array}$ & $\begin{array}{c}\text { Data } \\
\text { Source }\end{array}$ \\
\hline 294.0 & $4.31 \mathrm{E}+00$ & $1.20 E+00$ & cpt \\
\hline 293.0 & $5.63 E+00$ & $1.56 \mathrm{E}+00$ & cpt \\
\hline 292.0 & $4.95 E+00$ & $1.37 E+00$ & cpt \\
\hline 291.0 & $4.25 \mathrm{E}+00$ & $1.18 \mathrm{E}+00$ & cpt \\
\hline 290.0 & $4.55 \mathrm{E}+00$ & $1.27 E+00$ & $\mathrm{cpt}$ \\
\hline 289.0 & $5.74 E+00$ & $1.59 E+00$ & cpt \\
\hline 288.0 & $1.65 E+00$ & $4.59 \mathrm{E}-01$ & cpt \\
\hline 287.0 & $4.63 E-02$ & $1.29 \mathrm{E}-02$ & cpt \\
\hline 286.0 & 3.83E-03 & 1.06E-03 & $\mathrm{cpt}$ \\
\hline 285.0 & $1.58 \mathrm{E}-03$ & 4.40E-04 & cpt \\
\hline 284.0 & $1.57 E-03$ & $4.40 \mathrm{E}-04$ & cpt \\
\hline 283.0 & $5.63 E-03$ & $1.56 \mathrm{E}-03$ & $\mathrm{cpt}$ \\
\hline 282.0 & $1.16 \mathrm{E}+00$ & 3.23E-01 & cpt \\
\hline 281.0 & $7.24 \mathrm{E}-03$ & 2.01E-03 & cpt \\
\hline 280.0 & $1.71 E-03$ & $4.80 \mathrm{E}-04$ & cpt \\
\hline 279.0 & $1.48 E+01$ & $4.11 E+00$ & cpt \\
\hline 278.0 & $8.42 \mathrm{E}+01$ & $2.34 \mathrm{E}+01$ & cpt \\
\hline 277.0 & $2.90 \mathrm{E}-03$ & $8.00 E-04$ & cpt \\
\hline 276.0 & $1.52 E-03$ & $4.20 \mathrm{E}-04$ & cpt \\
\hline 275.0 & $1.71 \mathrm{E}-03$ & $4.70 \mathrm{E}-04$ & cpt \\
\hline 274.0 & $1.34 \mathrm{E}-03$ & $3.70 \mathrm{E}-04$ & $\mathrm{cpt}$ \\
\hline 273.0 & $1.93 \mathrm{E}-01$ & $5.36 \mathrm{E}-02$ & $\mathrm{cpt}$ \\
\hline 272.0 & $1.26 \mathrm{E}-03$ & $3.50 E-04$ & cpt \\
\hline 271.0 & $6.02 E+00$ & $1.67 \mathrm{E}+00$ & cpt \\
\hline 270.0 & $1.73 \mathrm{E}+01$ & $4.80 \mathrm{E}+00$ & cpt \\
\hline 269.0 & $1.20 E+01$ & $3.34 \mathrm{E}+00$ & cpt \\
\hline 268.0 & $5.79 E+00$ & $1.61 \mathrm{E}+00$ & $\mathrm{cpt}$ \\
\hline 267.0 & $8.07 E+00$ & $2.24 \mathrm{E}+00$ & $\mathrm{cpt}$ \\
\hline 266.0 & $9.52 \mathrm{E}+00$ & $2.64 E+00$ & cpt \\
\hline 265.0 & $5.51 E+00$ & $1.53 \mathrm{E}+00$ & cpt \\
\hline 264.0 & $6.28 \mathrm{E}+00$ & $1.74 \mathrm{E}+00$ & $\mathrm{cpt}$ \\
\hline 263.0 & $1.07 \mathrm{E}+01$ & $2.97 \mathrm{E}+00$ & cpt \\
\hline 262.0 & $6.82 \mathrm{E}+00$ & $1.90 \mathrm{E}+00$ & cpt \\
\hline 261.0 & $5.00 \mathrm{E}-04$ & $1.40 \mathrm{E}-04$ & $\mathrm{cpt}$ \\
\hline 260.0 & $4.60 \mathrm{E}-04$ & $1.30 \mathrm{E}-04$ & cpt \\
\hline 259.0 & $4.50 \mathrm{E}-04$ & $1.20 \mathrm{E}-04$ & cpt \\
\hline 258.0 & $4.30 E-04$ & $1.20 \mathrm{E}-04$ & cpt \\
\hline 257.0 & $4.20 \mathrm{E}-04$ & 1.20E-04 & $\mathrm{cpt}$ \\
\hline 256.0 & $4.60 \mathrm{E}-04$ & $1.30 \mathrm{E}-04$ & cpt \\
\hline 255.0 & $4.75 \mathrm{E}+00$ & $1.32 \mathrm{E}+00$ & $\mathrm{cpt}$ \\
\hline 254.0 & $8.60 \mathrm{E}-01$ & $2.39 \mathrm{E}-01$ & $\mathrm{cpt}$ \\
\hline 253.0 & $5.10 \mathrm{E}-04$ & $1.40 \mathrm{E}-04$ & $\mathrm{cpt}$ \\
\hline 252.0 & $7.80 \mathrm{E}-04$ & $2.20 E-04$ & $\mathrm{cpt}$ \\
\hline 251.0 & $5.10 \mathrm{E}-04$ & $1.40 \mathrm{E}-04$ & $\mathrm{cpt}$ \\
\hline 250.0 & $9.80 \mathrm{E}-04$ & $2.70 E-04$ & $\mathrm{cpt}$ \\
\hline 249.0 & $5.10 E-04$ & $1.40 E-04$ & $\mathrm{cpt}$ \\
\hline 248.0 & $5.00 \mathrm{E}-04$ & $1.40 \mathrm{E}-04$ & $\mathrm{cpt}$ \\
\hline 247.0 & $5.20 \mathrm{E}-04$ & $1.40 \mathrm{E}-04$ & $\mathrm{cpt}$ \\
\hline
\end{tabular}


Conductivity Data at RPT5

\begin{tabular}{|c|c|c|c|}
\hline $\begin{array}{l}\text { Elevation } \\
\text { (ft) }\end{array}$ & $\begin{array}{c}\mathrm{Kh} \\
\text { (ft/day) }\end{array}$ & $\begin{array}{c}\mathrm{Kv} \\
\text { (ft/day) }\end{array}$ & $\begin{array}{c}\text { Data } \\
\text { Source }\end{array}$ \\
\hline 246.0 & $4.90 \mathrm{E}-04$ & $1.40 \mathrm{E}-04$ & cpt \\
\hline 245.0 & $4.90 \mathrm{E}-04$ & $1.40 \mathrm{E}-04$ & cpt \\
\hline 244.0 & $5.00 \mathrm{E}-04$ & $1.40 \mathrm{E}-04$ & $\mathrm{cpt}$ \\
\hline 243.0 & $5.00 \mathrm{E}-04$ & $1.40 \mathrm{E}-04$ & cpt \\
\hline 242.0 & $4.80 E-04$ & 1.30E-04 & cpt \\
\hline 241.0 & $4.80 \mathrm{E}-04$ & $1.30 \mathrm{E}-04$ & $\mathrm{cpt}$ \\
\hline 240.0 & $4.90 \mathrm{E}-04$ & $1.40 \mathrm{E}-04$ & $\mathrm{cpt}$ \\
\hline 239.0 & $5.10 \mathrm{E}-04$ & $1.40 \mathrm{E}-04$ & $\mathrm{cpt}$ \\
\hline 238.0 & 5.00 E-04 & 1.40E-04 & $\mathrm{cpt}$ \\
\hline 237.0 & $5.10 \mathrm{E}-04$ & $1.40 \mathrm{E}-04$ & cpt \\
\hline 236.0 & $5.50 \mathrm{E}-04$ & $1.50 \mathrm{E}-04$ & $\mathrm{cpt}$ \\
\hline 235.0 & $5.00 \mathrm{E}-04$ & $1.40 \mathrm{E}-04$ & cpt \\
\hline 234.0 & $1.69 \mathrm{E}-01$ & $4.68 \mathrm{E}-02$ & $c p t$ \\
\hline 233.0 & $2.66 \mathrm{E}+00$ & $7.39 \mathrm{E}-01$ & $\mathrm{cpt}$ \\
\hline 232.0 & $1.26 \mathrm{E}+01$ & $3.50 E+00$ & cpt \\
\hline 231.0 & $7.74 E+00$ & $2.15 E+00$ & $\mathrm{cpt}$ \\
\hline 230.0 & $3.40 \mathrm{E}+00$ & 9.44E-01 & cpt \\
\hline 229.0 & $8.62 E+00$ & $2.40 \mathrm{E}+00$ & cpt \\
\hline 228.0 & $8.97 E-01$ & $2.49 \mathrm{E}-01$ & $\mathrm{cpt}$ \\
\hline 227.0 & $1.46 \mathrm{E}+00$ & $4.04 \mathrm{E}-01$ & cpt \\
\hline 226.0 & $5.31 \mathrm{E}+00$ & $1.47 E+00$ & cpt \\
\hline 225.0 & $9.77 E+00$ & $2.71 E+00$ & $\mathrm{cpt}$ \\
\hline 224.0 & $1.44 \mathrm{E}+01$ & $4.01 E+00$ & $\mathrm{cpt}$ \\
\hline 223.0 & $1.05 \mathrm{E}+01$ & $2.90 \mathrm{E}+00$ & $\mathrm{cpt}$ \\
\hline 222.0 & $1.31 \mathrm{E}+00$ & 3.63E-01 & cpt \\
\hline 221.0 & $6.90 \mathrm{E}-04$ & $1.90 \mathrm{E}-04$ & $\mathrm{cpt}$ \\
\hline 220.0 & $7.57 \mathrm{E}+00$ & $2.10 E+00$ & $\mathrm{cpt}$ \\
\hline 219.0 & $8.24 E+00$ & $2.29 \mathrm{E}+00$ & $\mathrm{cpt}$ \\
\hline 218.0 & $7.35 \mathrm{E}+00$ & $2.04 \mathrm{E}+00$ & cpt \\
\hline 217.0 & $7.68 \mathrm{E}+00$ & $2.13 E+00$ & $\mathrm{cpt}$ \\
\hline 216.0 & $7.50 \mathrm{E}+00$ & $2.08 \mathrm{E}+00$ & cpt \\
\hline 215.0 & $1.01 \mathrm{E}-03$ & $2.80 \mathrm{E}-04$ & $\mathrm{cpt}$ \\
\hline 214.0 & $1.05 E-03$ & $2.90 \mathrm{E}-04$ & cpt \\
\hline 213.0 & $8.81 E-03$ & $2.45 \mathrm{E}-03$ & cpt \\
\hline 212.0 & $1.19 E+01$ & $3.29 E+00$ & cpt \\
\hline 211.0 & $1.07 E+01$ & $2.97 \mathrm{E}+00$ & $\mathrm{cpt}$ \\
\hline 210.0 & $7.36 \mathrm{E}+00$ & $2.04 \mathrm{E}+00$ & $\mathrm{cpt}$ \\
\hline 209.0 & $1.29 \mathrm{E}+01$ & $3.59 \mathrm{E}+00$ & $\mathrm{cpt}$ \\
\hline 208.0 & $1.47 \mathrm{E}+01$ & $4.07 \mathrm{E}+00$ & cpt \\
\hline 207.0 & $1.51 \mathrm{E}+01$ & $4.20 \mathrm{E}+00$ & cpt \\
\hline 206.0 & $9.72 E+00$ & $2.70 \mathrm{E}+00$ & $\mathrm{cpt}$ \\
\hline 205.0 & $1.98 \mathrm{E}+00$ & $5.51 \mathrm{E}-01$ & cpt \\
\hline 204.0 & 1.60E-03 & $4.50 E-04$ & $\mathrm{cpt}$ \\
\hline 203.0 & 1.99E-01 & $5.52 E-02$ & cpt \\
\hline 202.0 & $2.67 E+01$ & $7.41 \mathrm{E}+00$ & $\mathrm{cpt}$ \\
\hline 201.0 & $2.84 E+01$ & $7.89 \mathrm{E}+00$ & cpt \\
\hline 200.0 & $4.34 E+01$ & $1.20 \mathrm{E}+01$ & cpt \\
\hline 199.0 & $5.24 E+01$ & $1.46 \mathrm{E}+01$ & $\mathrm{cpt}$ \\
\hline
\end{tabular}


Conductivity Data at RPT5

\begin{tabular}{|c|c|c|c|}
\hline $\begin{array}{c}\text { Elevation } \\
\text { (ft) }\end{array}$ & $\begin{array}{c}\mathrm{Kh} \\
\text { (ft/day) }\end{array}$ & $\begin{array}{c}\mathrm{Kv} \\
\text { (ft/day) }\end{array}$ & $\begin{array}{c}\text { Data } \\
\text { Source }\end{array}$ \\
\hline 198.0 & $4.84 \mathrm{E}+01$ & $1.35 \mathrm{E}+01$ & $\mathrm{cpt}$ \\
\hline 197.0 & $3.52 \mathrm{E}+01$ & $9.78 \mathrm{E}+00$ & $\mathrm{cpt}$ \\
\hline 196.0 & $3.81 \mathrm{E}+01$ & $1.06 \mathrm{E}+01$ & $\mathrm{cpt}$ \\
\hline 195.0 & $3.84 \mathrm{E}+01$ & $1.07 \mathrm{E}+01$ & $\mathrm{cpt}$ \\
\hline 194.0 & $4.38 \mathrm{E}+01$ & $1.22 \mathrm{E}+01$ & $\mathrm{cpt}$ \\
\hline 193.0 & $4.37 \mathrm{E}+01$ & $1.21 \mathrm{E}+01$ & $\mathrm{cpt}$ \\
\hline 192.0 & $1.42 \mathrm{E}-01$ & $3.94 \mathrm{E}-02$ & $\mathrm{cpt}$ \\
\hline 191.0 & $1.80 \mathrm{E}-03$ & $5.00 \mathrm{E}-04$ & $\mathrm{cpt}$ \\
\hline 190.0 & $3.43 \mathrm{E}-03$ & $9.50 \mathrm{E}-04$ & $\mathrm{cpt}$ \\
\hline 189.0 & $8.17 \mathrm{E}-02$ & $2.27 \mathrm{E}-02$ & $\mathrm{cpt}$ \\
\hline 188.0 & $2.86 \mathrm{E}+01$ & $7.94 \mathrm{E}+00$ & $\mathrm{cpt}$ \\
\hline 187.0 & $3.14 \mathrm{E}+01$ & $8.71 \mathrm{E}+00$ & $\mathrm{cpt}$ \\
\hline 186.0 & $1.61 \mathrm{E}-03$ & $4.50 \mathrm{E}-04$ & $\mathrm{cpt}$ \\
\hline 185.0 & $5.98 \mathrm{E}+01$ & $1.66 \mathrm{E}+01$ & $\mathrm{cpt}$ \\
\hline 184.0 & $1.00 \mathrm{E}+02$ & $2.78 \mathrm{E}+01$ & $\mathrm{cpt}$ \\
\hline 183.0 & $1.00 \mathrm{E}+02$ & $2.78 \mathrm{E}+01$ & $\mathrm{cpt}$ \\
\hline 182.0 & $1.00 \mathrm{E}+02$ & $2.78 \mathrm{E}+01$ & $\mathrm{cpt}$ \\
\hline 181.0 & $6.95 \mathrm{E}+01$ & $1.93 \mathrm{E}+01$ & $\mathrm{cpt}$ \\
\hline 180.0 & $4.26 \mathrm{E}+01$ & $1.18 \mathrm{E}+01$ & $\mathrm{cpt}$ \\
\hline 179.0 & $2.61 \mathrm{E}+01$ & $7.24 \mathrm{E}+00$ & $\mathrm{cpt}$ \\
\hline 178.0 & $2.65 \mathrm{E}+01$ & $7.36 \mathrm{E}+00$ & $\mathrm{cpt}$ \\
\hline 177.0 & $3.01 \mathrm{E}+01$ & $8.35 \mathrm{E}+00$ & $\mathrm{cpt}$ \\
\hline 176.0 & $2.66 \mathrm{E}+01$ & $7.39 \mathrm{E}+00$ & $\mathrm{cpt}$ \\
\hline 175.0 & $5.61 \mathrm{E}+01$ & $1.56 \mathrm{E}+01$ & $\mathrm{cpt}$ \\
\hline 174.0 & $5.28 \mathrm{E}-01$ & $1.47 \mathrm{E}-01$ & $\mathrm{cpt}$ \\
\hline 173.0 & $8.10 \mathrm{E}-04$ & $2.30 \mathrm{E}-04$ & $\mathrm{cpt}$ \\
\hline 172.0 & $7.40 \mathrm{E}-04$ & $2.10 \mathrm{E}-04$ & $\mathrm{cpt}$ \\
\hline 171.0 & $7.60 \mathrm{E}-04$ & $2.10 \mathrm{E}-04$ & $\mathrm{cpt}$ \\
\hline 170.0 & $6.44 \mathrm{E}-02$ & $1.79 \mathrm{E}-02$ & $\mathrm{cpt}$ \\
\hline 169.0 & $4.69 \mathrm{E}+01$ & $1.30 \mathrm{E}+01$ & $\mathrm{cpt}$ \\
\hline 168.0 & $4.38 \mathrm{E}+01$ & $1.22 \mathrm{E}+01$ & $\mathrm{cpt}$ \\
\hline 167.0 & $4.78 \mathrm{E}+01$ & $1.33 \mathrm{E}+01$ & $\mathrm{cpt}$ \\
\hline 166.0 & $1.00 \mathrm{E}+02$ & $2.78 \mathrm{E}+01$ & $\mathrm{cpt}$ \\
\hline 165.0 & $1.00 \mathrm{E}+02$ & $2.78 \mathrm{E}+01$ & $\mathrm{cpt}$ \\
\hline 164.0 & $8.71 \mathrm{E}-02$ & $2.42 \mathrm{E}-02$ & $\mathrm{cpt}$ \\
\hline 163.0 & $6.00 \mathrm{E}-04$ & $1.70 \mathrm{E}-04$ & $\mathrm{cpt}$ \\
\hline 162.0 & $1.15 \mathrm{E}-03$ & $3.20 \mathrm{E}-04$ & $\mathrm{cpt}$ \\
\hline 161.0 & $2.14 \mathrm{E}-02$ & $5.94 \mathrm{E}-03$ & $\mathrm{cpt}$ \\
\hline 160.0 & $7.94 \mathrm{E}+01$ & $2.21 \mathrm{E}+01$ & $\mathrm{cpt}$ \\
\hline 159.0 & $5.67 \mathrm{E}+01$ & $1.57 \mathrm{E}+01$ & $\mathrm{cpt}$ \\
\hline 158.0 & $6.66 \mathrm{E}+01$ & $1.85 \mathrm{E}+01$ & $\mathrm{cpt}$ \\
\hline 157.0 & $7.73 \mathrm{E}-03$ & $2.15 \mathrm{E}-03$ & $\mathrm{cpt}$ \\
\hline 156.0 & $6.89 \mathrm{E}-03$ & $1.91 \mathrm{E}-03$ & $\mathrm{cpt}$ \\
\hline 155.0 & $1.23 \mathrm{E}-03$ & $3.40 \mathrm{E}-04$ & $\mathrm{cpt}$ \\
\hline 154.0 & $8.30 \mathrm{E}-04$ & $2.30 \mathrm{E}-04$ & $\mathrm{cpt}$ \\
\hline 153.0 & $9.00 \mathrm{E}-04$ & $2.50 \mathrm{E}-04$ & $\mathrm{cpt}$ \\
\hline 152.0 & $8.60 \mathrm{E}-04$ & $2.40 \mathrm{E}-04$ & $\mathrm{cpt}$ \\
\hline 151.0 & $3.57 \mathrm{E}-01$ & $9.92 \mathrm{E}-02$ & $\mathrm{cpt}$ \\
\hline & & & \\
\hline
\end{tabular}


Conductivity Data at RPT5

\begin{tabular}{|c|c|c|c|}
\hline $\begin{array}{c}\text { Elevation } \\
\text { (ft) }\end{array}$ & $\begin{array}{c}\text { Kh } \\
\text { (ft/day) }\end{array}$ & $\begin{array}{c}\text { Kv } \\
\text { (ft/day) }\end{array}$ & $\begin{array}{c}\text { Data } \\
\text { Source }\end{array}$ \\
\hline 150.0 & $7.45 \mathrm{E}-03$ & $2.07 \mathrm{E}-03$ & $\mathrm{cpt}$ \\
\hline 149.0 & $6.69 \mathrm{E}+01$ & $1.86 \mathrm{E}+01$ & $\mathrm{cpt}$ \\
\hline 148.0 & $3.35 \mathrm{E}+01$ & $9.31 \mathrm{E}+00$ & $\mathrm{cpt}$ \\
\hline 147.0 & $4.34 \mathrm{E}+00$ & $1.20 \mathrm{E}+00$ & $\mathrm{cpt}$ \\
\hline 146.0 & $1.15 \mathrm{E}+01$ & $3.18 \mathrm{E}+00$ & $\mathrm{cpt}$ \\
\hline 145.0 & $1.37 \mathrm{E}+00$ & $3.80 \mathrm{E}-01$ & $\mathrm{cpt}$ \\
\hline 144.0 & $4.72 \mathrm{E}-03$ & $1.31 \mathrm{E}-03$ & $\mathrm{cpt}$ \\
\hline 143.0 & $5.52 \mathrm{E}+00$ & $1.53 \mathrm{E}+00$ & $\mathrm{cpt}$ \\
\hline 142.0 & $1.00 \mathrm{E}+02$ & $2.78 \mathrm{E}+01$ & $\mathrm{cpt}$ \\
\hline 141.0 & $4.33 \mathrm{E}+01$ & $1.20 \mathrm{E}+01$ & $\mathrm{cpt}$ \\
\hline 140.0 & $3.58 \mathrm{E}+01$ & $9.94 \mathrm{E}+00$ & $\mathrm{cpt}$ \\
\hline 139.0 & $3.34 \mathrm{E}+01$ & $9.28 \mathrm{E}+00$ & $\mathrm{cpt}$ \\
\hline 138.0 & $6.17 \mathrm{E}+01$ & $1.71 \mathrm{E}+01$ & $\mathrm{cpt}$ \\
\hline 137.0 & $1.00 \mathrm{E}+02$ & $2.78 \mathrm{E}+01$ & $\mathrm{cpt}$ \\
\hline 136.0 & $1.00 \mathrm{E}+02$ & $2.78 \mathrm{E}+01$ & $\mathrm{cpt}$ \\
\hline 135.0 & $1.00 \mathrm{E}+02$ & $2.78 \mathrm{E}+01$ & $\mathrm{cpt}$ \\
\hline 134.0 & $1.00 \mathrm{E}+02$ & $2.78 \mathrm{E}+01$ & $\mathrm{cpt}$ \\
\hline 133.0 & $1.00 \mathrm{E}+02$ & $2.78 \mathrm{E}+01$ & $\mathrm{cpt}$ \\
\hline
\end{tabular}


Conductivity Data at RPT6

\begin{tabular}{|c|c|c|c|}
\hline $\begin{array}{c}\text { Elevation } \\
\text { (ft) }\end{array}$ & $\begin{array}{c}\text { Kh } \\
\text { (ft/day) }\end{array}$ & $\begin{array}{c}\text { Kv } \\
\text { (ft/day) }\end{array}$ & $\begin{array}{c}\text { Data } \\
\text { Source }\end{array}$ \\
\hline 297.4 & $3.59 \mathrm{E}+00$ & $9.96 \mathrm{E}-01$ & $\mathrm{cpt}$ \\
\hline 296.4 & $4.12 \mathrm{E}+00$ & $1.14 \mathrm{E}+00$ & $\mathrm{cpt}$ \\
\hline 295.4 & $4.00 \mathrm{E}+00$ & $1.11 \mathrm{E}+00$ & $\mathrm{cpt}$ \\
\hline 294.4 & $4.49 \mathrm{E}+00$ & $1.25 \mathrm{E}+00$ & $\mathrm{cpt}$ \\
\hline 293.4 & $5.71 \mathrm{E}+00$ & $1.59 \mathrm{E}+00$ & $\mathrm{cpt}$ \\
\hline 292.4 & $7.16 \mathrm{E}-03$ & $1.99 \mathrm{E}-03$ & $\mathrm{cpt}$ \\
\hline 291.4 & $1.76 \mathrm{E}-03$ & $4.90 \mathrm{E}-04$ & $\mathrm{cpt}$ \\
\hline 290.4 & $1.80 \mathrm{E}-03$ & $5.00 \mathrm{E}-04$ & $\mathrm{cpt}$ \\
\hline 289.4 & $1.90 \mathrm{E}-03$ & $5.30 \mathrm{E}-04$ & $\mathrm{cpt}$ \\
\hline 288.4 & $3.05 \mathrm{E}-03$ & $8.50 \mathrm{E}-04$ & $\mathrm{cpt}$ \\
\hline 287.4 & $2.98 \mathrm{E}-03$ & $8.30 \mathrm{E}-04$ & $\mathrm{cpt}$ \\
\hline 286.4 & $1.17 \mathrm{E}-02$ & $3.26 \mathrm{E}-03$ & $\mathrm{cpt}$ \\
\hline 285.4 & $3.17 \mathrm{E}-03$ & $8.80 \mathrm{E}-04$ & $\mathrm{cpt}$ \\
\hline 284.4 & $2.79 \mathrm{E}-03$ & $7.80 \mathrm{E}-04$ & $\mathrm{cpt}$ \\
\hline 283.4 & $2.54 \mathrm{E}-03$ & $7.10 \mathrm{E}-04$ & $\mathrm{cpt}$ \\
\hline 282.4 & $1.99 \mathrm{E}-03$ & $5.50 \mathrm{E}-04$ & $\mathrm{cpt}$ \\
\hline 281.4 & $1.89 \mathrm{E}-03$ & $5.30 \mathrm{E}-04$ & $\mathrm{cpt}$ \\
\hline 280.4 & $1.50 \mathrm{E}-03$ & $4.20 \mathrm{E}-04$ & $\mathrm{cpt}$ \\
\hline 279.4 & $1.95 \mathrm{E}-03$ & $5.40 \mathrm{E}-04$ & $\mathrm{cpt}$ \\
\hline 278.4 & $1.66 \mathrm{E}-03$ & $4.60 \mathrm{E}-04$ & $\mathrm{cpt}$ \\
\hline 277.4 & $2.24 \mathrm{E}-03$ & $6.20 \mathrm{E}-04$ & $\mathrm{cpt}$ \\
\hline 276.4 & $2.82 \mathrm{E}-03$ & $7.80 \mathrm{E}-04$ & $\mathrm{cpt}$ \\
\hline 275.4 & $2.85 \mathrm{E}-03$ & $7.90 \mathrm{E}-04$ & $\mathrm{cpt}$ \\
\hline 274.4 & $7.15 \mathrm{E}-02$ & $1.99 \mathrm{E}-02$ & $\mathrm{cpt}$ \\
\hline 273.4 & $1.94 \mathrm{E}-01$ & $5.38 \mathrm{E}-02$ & $\mathrm{cpt}$ \\
\hline 272.4 & $6.30 \mathrm{E}+00$ & $1.75 \mathrm{E}+00$ & $\mathrm{cpt}$ \\
\hline 271.4 & $6.26 \mathrm{E}+00$ & $1.74 \mathrm{E}+00$ & $\mathrm{cpt}$ \\
\hline 270.4 & $1.74 \mathrm{E}+00$ & $4.84 \mathrm{E}-01$ & $\mathrm{cpt}$ \\
\hline 269.4 & $5.71 \mathrm{E}+00$ & $1.59 \mathrm{E}+00$ & $\mathrm{cpt}$ \\
\hline 268.4 & $5.41 \mathrm{E}+00$ & $1.50 \mathrm{E}+00$ & $\mathrm{cpt}$ \\
\hline 267.4 & $6.03 \mathrm{E}+00$ & $1.67 \mathrm{E}+00$ & $\mathrm{cpt}$ \\
\hline 266.4 & $5.76 \mathrm{E}+00$ & $1.60 \mathrm{E}+00$ & $\mathrm{cpt}$ \\
\hline 265.4 & $5.11 \mathrm{E}+00$ & $1.42 \mathrm{E}+00$ & $\mathrm{cpt}$ \\
\hline 264.4 & $6.85 \mathrm{E}+00$ & $1.90 \mathrm{E}+00$ & $\mathrm{cpt}$ \\
\hline 263.4 & $6.65 \mathrm{E}+00$ & $1.85 \mathrm{E}+00$ & $\mathrm{cpt}$ \\
\hline 262.4 & $8.67 \mathrm{E}+00$ & $2.41 \mathrm{E}+00$ & $\mathrm{cpt}$ \\
\hline 261.4 & $1.52 \mathrm{E}+01$ & $4.21 \mathrm{E}+00$ & $\mathrm{cpt}$ \\
\hline 260.4 & $1.69 \mathrm{E}+01$ & $4.70 \mathrm{E}+00$ & $\mathrm{cpt}$ \\
\hline 259.4 & $1.25 \mathrm{E}+01$ & $3.48 \mathrm{E}+00$ & $\mathrm{cpt}$ \\
\hline 258.4 & $1.29 \mathrm{E}+01$ & $3.59 \mathrm{E}+00$ & $\mathrm{cpt}$ \\
\hline 257.4 & $3.31 \mathrm{E}-02$ & $9.20 \mathrm{E}-03$ & $\mathrm{cpt}$ \\
\hline 256.4 & $4.29 \mathrm{E}-01$ & $1.19 \mathrm{E}-01$ & $\mathrm{cpt}$ \\
\hline 255.4 & $6.43 \mathrm{E}+00$ & $1.79 \mathrm{E}+00$ & $\mathrm{cpt}$ \\
\hline 253.4 & $7.37 \mathrm{E}+00$ & $2.05 \mathrm{E}+00$ & $\mathrm{cpt}$ \\
\hline 251.4 & $6.42 \mathrm{E}+00$ & $1.78 \mathrm{E}+00$ & $\mathrm{cpt}$ \\
\hline 250.4 & $1.00 \mathrm{E}+01$ & $2.78 \mathrm{E}+00$ & $\mathrm{cpt}$ \\
\hline & & & \\
\hline
\end{tabular}


Conductivity Data at RPT6

\begin{tabular}{|c|c|c|c|}
\hline $\begin{array}{c}\text { Elevation } \\
\text { (ft) }\end{array}$ & $\begin{array}{c}\mathrm{Kh} \\
\text { (ft/day) }\end{array}$ & $\begin{array}{c}\text { Kv } \\
\text { (ft/day) }\end{array}$ & $\begin{array}{c}\text { Data } \\
\text { Source }\end{array}$ \\
\hline 249.4 & $2.00 E+01$ & $5.55 E+00$ & cpt \\
\hline 248.4 & $2.19 \mathrm{E}+01$ & $6.09 \mathrm{E}+00$ & $\mathrm{cpt}$ \\
\hline 247.4 & $2.17 E+01$ & $6.04 \mathrm{E}+00$ & cpt \\
\hline 246.4 & $1.85 \mathrm{E}+01$ & $5.15 \mathrm{E}+00$ & $\mathrm{cpt}$ \\
\hline 245.4 & $1.56 \mathrm{E}+01$ & $4.34 \mathrm{E}+00$ & cpt \\
\hline 244.4 & $1.59 E+01$ & $4.43 E+00$ & $\mathrm{cpt}$ \\
\hline 243.4 & $8.32 \mathrm{E}+00$ & $2.31 \mathrm{E}+00$ & $\mathrm{cpt}$ \\
\hline 242.4 & 7.00 E-04 & $1.90 \mathrm{E}-04$ & $\mathrm{cpt}$ \\
\hline 241.4 & 4.91E-01 & $1.36 \mathrm{E}-01$ & $\mathrm{cpt}$ \\
\hline 240.4 & $3.79 \mathrm{E}+00$ & $1.05 \mathrm{E}+00$ & $\mathrm{cpt}$ \\
\hline 239.4 & $7.40 \mathrm{E}+00$ & $2.06 \mathrm{E}+00$ & $\overline{c p t}$ \\
\hline 238.4 & $1.54 \mathrm{E}+01$ & $4.27 E+00$ & $c p t$ \\
\hline 237.4 & $2.38 \mathrm{E}+01$ & $6.60 \mathrm{E}+00$ & $\mathrm{cpt}$ \\
\hline 236.4 & $2.38 \mathrm{E}+01$ & $6.60 \mathrm{E}+00$ & $\mathrm{cpt}$ \\
\hline 235.4 & $2.30 \mathrm{E}+01$ & $6.38 \mathrm{E}+00$ & $\mathrm{cpt}$ \\
\hline 234.4 & $2.34 \mathrm{E}+01$ & $6.50 \mathrm{E}+00$ & cpt \\
\hline 233.4 & $2.09 E+01$ & $5.81 \mathrm{E}+00$ & $\mathrm{cpt}$ \\
\hline 232.4 & $2.08 E+01$ & $5.79 E+00$ & cpt \\
\hline 231.4 & $2.13 E+01$ & $5.92 \mathrm{E}+00$ & $\mathrm{cpt}$ \\
\hline 230.4 & $1.38 \mathrm{E}+01$ & $3.82 \mathrm{E}+00$ & cpt \\
\hline 229.4 & $5.26 \mathrm{E}-03$ & $1.46 \mathrm{E}-03$ & cpt \\
\hline 228.4 & $1.55 \mathrm{E}-03$ & $4.30 E-04$ & $\mathrm{cpt}$ \\
\hline 226.9 & $1.22 \mathrm{E}-02$ & $3.40 \mathrm{E}-03$ & perm \\
\hline 225.9 & $5.51 \mathrm{E}-02$ & $1.53 \mathrm{E}-02$ & perm \\
\hline 224.4 & $5.82 E-01$ & $1.62 \mathrm{E}-01$ & $\mathrm{cpt}$ \\
\hline 223.4 & $1.52 \mathrm{E}+01$ & $4.22 \mathrm{E}+00$ & $\mathrm{cpt}$ \\
\hline 222.4 & $1.40 \mathrm{E}+01$ & $3.89 \mathrm{E}+00$ & $\mathrm{cpt}$ \\
\hline 221.4 & $1.32 E-02$ & $3.67 \mathrm{E}-03$ & $\mathrm{cpt}$ \\
\hline 220.4 & $2.30 \mathrm{E}+01$ & $6.39 \mathrm{E}+00$ & $\mathrm{cpt}$ \\
\hline 219.4 & $7.59 \mathrm{E}+01$ & $2.11 E+01$ & $\mathrm{cpt}$ \\
\hline 218.4 & $4.15 \mathrm{E}+01$ & $1.15 \mathrm{E}+01$ & cpt \\
\hline 216.9 & $6.23 E+00$ & $1.73 E+00$ & perm \\
\hline 215.4 & $1.76 \mathrm{E}+01$ & $4.88 \mathrm{E}+00$ & $\mathrm{cpt}$ \\
\hline 214.4 & $1.73 E+01$ & $4.81 \mathrm{E}+00$ & $c p t$ \\
\hline 213.4 & $9.42 E+00$ & $2.62 E+00$ & $\mathrm{cpt}$ \\
\hline 212.4 & $1.64 \mathrm{E}+01$ & $4.57 \mathrm{E}+00$ & $\mathrm{cpt}$ \\
\hline 211.4 & $1.89 \mathrm{E}+01$ & $5.25 E+00$ & $\mathrm{cpt}$ \\
\hline 210.4 & $1.83 \mathrm{E}+01$ & $5.09 \mathrm{E}+00$ & $\mathrm{cpt}$ \\
\hline 209.4 & $1.63 \mathrm{E}+01$ & $4.54 \mathrm{E}+00$ & $\mathrm{cpt}$ \\
\hline 208.4 & $1.78 \mathrm{E}+01$ & $4.95 E+00$ & cpt \\
\hline 207.4 & $1.91 \mathrm{E}+01$ & $5.32 \mathrm{E}+00$ & $\mathrm{cpt}$ \\
\hline 206.4 & $2.27 \mathrm{E}+01$ & $6.30 \mathrm{E}+00$ & cpt \\
\hline 205.4 & $2.67 \mathrm{E}+01$ & $7.43 E+00$ & cpt \\
\hline 204.4 & $3.04 \mathrm{E}+01$ & $8.43 E+00$ & cpt \\
\hline 203.4 & $4.06 \mathrm{E}+01$ & $1.13 E+01$ & cpt \\
\hline 202.4 & $5.73 E+01$ & $1.59 \mathrm{E}+01$ & cpt \\
\hline 201.4 & $5.50 \mathrm{E}+01$ & $1.53 E+01$ & $\mathrm{cpt}$ \\
\hline 200.4 & $2.31 E+01$ & $6.41 \mathrm{E}+00$ & $\mathrm{cpt}$ \\
\hline
\end{tabular}


Conductivity Data at RPT6

\begin{tabular}{|c|c|c|c|}
\hline $\begin{array}{c}\text { Elevation } \\
\text { (ft) }\end{array}$ & $\begin{array}{c}\text { Kh } \\
\text { (ft/day) }\end{array}$ & $\begin{array}{c}\mathrm{Kv} \\
\text { (ft/day) }\end{array}$ & $\begin{array}{c}\text { Data } \\
\text { Source }\end{array}$ \\
\hline 199.4 & $1.43 \mathrm{E}-03$ & 4.00E-04 & $\mathrm{cpt}$ \\
\hline 198.4 & $5.40 \mathrm{E}-04$ & $1.50 E-04$ & $\mathrm{cpt}$ \\
\hline 197.4 & $7.90 \mathrm{E}-04$ & $2.20 \mathrm{E}-04$ & cpt \\
\hline 196.4 & $1.17 \mathrm{E}+01$ & $3.25 \mathrm{E}+00$ & cpt \\
\hline 195.4 & $2.23 \mathrm{E}+01$ & $6.18 E+00$ & cpt \\
\hline 194.4 & $2.86 E+01$ & $7.93 E+00$ & cpt \\
\hline 193.4 & $4.27 E+01$ & $1.19 \mathrm{E}+01$ & $\mathrm{cpt}$ \\
\hline 192.4 & $4.02 E+01$ & $1.12 E+01$ & $\mathrm{cpt}$ \\
\hline 191.4 & $1.15 \mathrm{E}+01$ & $3.20 E+00$ & cpt \\
\hline 190.4 & $8.30 \mathrm{E}-04$ & $2.30 E-04$ & $\mathrm{cpt}$ \\
\hline 189.4 & $8.29 E-03$ & $2.30 E-03$ & $\mathrm{cpt}$ \\
\hline 188.4 & 7.70 E-04 & $2.20 E-04$ & cpt \\
\hline 187.4 & $5.90 \mathrm{E}-04$ & $1.60 \mathrm{E}-04$ & $\mathrm{cpt}$ \\
\hline 186.4 & $8.60 E-04$ & $2.40 E-04$ & cpt \\
\hline 185.4 & $7.70 \mathrm{E}-04$ & $2.10 \mathrm{E}-04$ & $\mathrm{cpt}$ \\
\hline 184.4 & $8.80 E-04$ & $2.40 E-04$ & $\mathrm{cpt}$ \\
\hline 183.4 & $1.03 E-03$ & $2.90 \mathrm{E}-04$ & cpt \\
\hline 181.9 & $2.25 E-04$ & $6.24 E-05$ & perm \\
\hline 180.9 & $4.50 \mathrm{E}-04$ & $1.25 \mathrm{E}-04$ & perm \\
\hline 179.4 & $4.98 \mathrm{E}-02$ & 1.38E-02 & $\mathrm{cpt}$ \\
\hline 178.4 & $1.78 E-01$ & 4.95E-02 & $\mathrm{cpt}$ \\
\hline 177.4 & $1.16 \mathrm{E}-03$ & 3.20 E-04 & cpt \\
\hline 176.4 & $1.03 \mathrm{E}-03$ & $2.90 \mathrm{E}-04$ & $\mathrm{cpt}$ \\
\hline 175.4 & $9.00 \mathrm{E}-04$ & $2.50 \mathrm{E}-04$ & cpt \\
\hline 174.4 & $1.62 \mathrm{E}+01$ & $4.50 E+00$ & $\mathrm{cpt}$ \\
\hline 173.4 & $1.25 \mathrm{E}-02$ & $3.47 \mathrm{E}-03$ & $\mathrm{cpt}$ \\
\hline 172.4 & $5.21 \mathrm{E}-03$ & $1.45 \mathrm{E}-03$ & $\mathrm{cpt}$ \\
\hline 171.4 & $3.64 \mathrm{E}-03$ & $1.01 \mathrm{E}-03$ & cpt \\
\hline 169.9 & $1.53 \mathrm{E}+01$ & $4.25 E+00$ & perm \\
\hline 168.4 & $5.86 \mathrm{E}+00$ & $1.63 E+00$ & $\mathrm{cpt}$ \\
\hline 167.4 & $4.05 E+01$ & $1.13 \mathrm{E}+01$ & $\mathrm{cpt}$ \\
\hline 166.4 & $2.89 E+01$ & $8.04 E+00$ & cpt \\
\hline 165.4 & $1.64 \mathrm{E}+01$ & $4.56 \mathrm{E}+00$ & $\mathrm{cpt}$ \\
\hline 164.4 & $5.42 \mathrm{E}+01$ & $1.51 \mathrm{E}+01$ & $\mathrm{cpt}$ \\
\hline 163.4 & $1.00 \mathrm{E}+02$ & $2.78 E+01$ & $\mathrm{cpt}$ \\
\hline 162.4 & $1.00 E+02$ & $2.78 E+01$ & $\mathrm{cpt}$ \\
\hline 161.4 & $2.85 E+01$ & $7.90 \mathrm{E}+00$ & cpt \\
\hline 160.4 & $1.61 \mathrm{E}+01$ & $4.46 \mathrm{E}+00$ & $\mathrm{cpt}$ \\
\hline 159.4 & $5.05 E+01$ & $1.40 E+01$ & cpt \\
\hline 158.4 & $1.00 \mathrm{E}+02$ & $2.78 \mathrm{E}+01$ & cpt \\
\hline 157.4 & $8.91 E+01$ & $2.48 E+01$ & cpt \\
\hline 156.4 & $4.42 E+01$ & $1.23 E+01$ & $\mathrm{cpt}$ \\
\hline 155.4 & $1.20 \mathrm{E}-01$ & 3.32E-02 & cpt \\
\hline 154.4 & $6.88 E+00$ & $1.91 E+00$ & cpt \\
\hline 153.4 & $7.76 \mathrm{E}+00$ & $2.16 E+00$ & cpt \\
\hline 152.4 & $3.58 \mathrm{E}-03$ & $9.90 E-04$ & $\mathrm{cpt}$ \\
\hline 151.4 & $5.76 \mathrm{E}-01$ & $1.60 \mathrm{E}-01$ & cpt \\
\hline 150.4 & $1.19 \mathrm{E}+01$ & $3.31 E+00$ & $\mathrm{cpt}$ \\
\hline
\end{tabular}


Conductivity Data at RPT6

\begin{tabular}{|c|c|c|c|}
\hline $\begin{array}{c}\text { Elevation } \\
\text { (ft) }\end{array}$ & $\begin{array}{c}\text { Kh } \\
\text { (ft/day) }\end{array}$ & $\begin{array}{c}\text { Kv } \\
\text { (ft/day) }\end{array}$ & $\begin{array}{c}\text { Data } \\
\text { Source }\end{array}$ \\
\hline 149.4 & $6.00 \mathrm{E}-04$ & $1.70 \mathrm{E}-04$ & $\mathrm{cpt}$ \\
\hline 148.4 & $1.28 \mathrm{E}+01$ & $3.56 \mathrm{E}+00$ & $\mathrm{cpt}$ \\
\hline 147.4 & $3.39 \mathrm{E}-03$ & $9.40 \mathrm{E}-04$ & $\mathrm{cpt}$ \\
\hline 146.4 & $7.42 \mathrm{E}+00$ & $2.06 \mathrm{E}+00$ & $\mathrm{cpt}$ \\
\hline 145.4 & $1.20 \mathrm{E}+01$ & $3.34 \mathrm{E}+00$ & $\mathrm{cpt}$ \\
\hline 144.4 & $1.39 \mathrm{E}+01$ & $3.87 \mathrm{E}+00$ & $\mathrm{cpt}$ \\
\hline 143.4 & $3.30 \mathrm{E}-01$ & $9.18 \mathrm{E}-02$ & $\mathrm{cpt}$ \\
\hline 142.4 & $5.50 \mathrm{E}-04$ & $1.50 \mathrm{E}-04$ & $\mathrm{cpt}$ \\
\hline 141.4 & $4.20 \mathrm{E}-04$ & $1.20 \mathrm{E}-04$ & $\mathrm{cpt}$ \\
\hline 140.4 & $5.67 \mathrm{E}-02$ & $1.58 \mathrm{E}-02$ & $\mathrm{cpt}$ \\
\hline 139.4 & $4.80 \mathrm{E}-04$ & $1.30 \mathrm{E}-04$ & $\mathrm{cpt}$ \\
\hline 138.4 & $6.40 \mathrm{E}-04$ & $1.80 \mathrm{E}-04$ & $\mathrm{cpt}$ \\
\hline 137.4 & $8.80 \mathrm{E}-04$ & $2.50 \mathrm{E}-04$ & $\mathrm{cpt}$ \\
\hline 136.4 & $1.14 \mathrm{E}+01$ & $3.18 \mathrm{E}+00$ & $\mathrm{cpt}$ \\
\hline 135.4 & $1.76 \mathrm{E}+01$ & $4.89 \mathrm{E}+00$ & $\mathrm{cpt}$ \\
\hline 134.4 & $1.26 \mathrm{E}-02$ & $3.50 \mathrm{E}-03$ & $\mathrm{cpt}$ \\
\hline
\end{tabular}


Conductivity Data at RPT7

\begin{tabular}{|c|c|c|c|}
\hline $\begin{array}{c}\text { Elevation } \\
\text { (ft) }\end{array}$ & $\begin{array}{c}\text { Kh } \\
\text { (ft/day) }\end{array}$ & $\begin{array}{c}\text { Kv } \\
\text { (ft/day) }\end{array}$ & $\begin{array}{c}\text { Data } \\
\text { Source }\end{array}$ \\
\hline 308.0 & $6.10 \mathrm{E}+00$ & $1.70 \mathrm{E}+00$ & $\mathrm{cpt}$ \\
\hline 307.0 & $2.18 \mathrm{E}+01$ & $6.06 \mathrm{E}+00$ & $\mathrm{cpt}$ \\
\hline 306.0 & $6.15 \mathrm{E}+00$ & $1.71 \mathrm{E}+00$ & $\mathrm{cpt}$ \\
\hline 305.0 & $5.57 \mathrm{E}+00$ & $1.55 \mathrm{E}+00$ & $\mathrm{cpt}$ \\
\hline 304.0 & $1.42 \mathrm{E}+01$ & $3.95 \mathrm{E}+00$ & $\mathrm{cpt}$ \\
\hline 303.0 & $1.49 \mathrm{E}+01$ & $4.15 \mathrm{E}+00$ & $\mathrm{cpt}$ \\
\hline 302.0 & $5.68 \mathrm{E}-02$ & $1.58 \mathrm{E}-02$ & $\mathrm{cpt}$ \\
\hline 301.0 & $9.87 \mathrm{E}-02$ & $2.74 \mathrm{E}-02$ & $\mathrm{cpt}$ \\
\hline 300.0 & $1.77 \mathrm{E}-02$ & $4.92 \mathrm{E}-03$ & $\mathrm{cpt}$ \\
\hline 299.0 & $7.94 \mathrm{E}-02$ & $2.20 \mathrm{E}-02$ & $\mathrm{cpt}$ \\
\hline 298.0 & $1.18 \mathrm{E}-02$ & $3.26 \mathrm{E}-03$ & $\mathrm{cpt}$ \\
\hline 297.0 & $2.28 \mathrm{E}-03$ & $6.30 \mathrm{E}-04$ & $\mathrm{cpt}$ \\
\hline 296.0 & $1.06 \mathrm{E}-03$ & $3.00 \mathrm{E}-04$ & $\mathrm{cpt}$ \\
\hline 295.0 & $1.50 \mathrm{E}-03$ & $4.20 \mathrm{E}-04$ & $\mathrm{cpt}$ \\
\hline 294.0 & $1.65 \mathrm{E}-03$ & $4.60 \mathrm{E}-04$ & $\mathrm{cpt}$ \\
\hline 293.0 & $1.72 \mathrm{E}-03$ & $4.80 \mathrm{E}-04$ & $\mathrm{cpt}$ \\
\hline 292.0 & $8.05 \mathrm{E}+00$ & $2.24 \mathrm{E}+00$ & $\mathrm{cpt}$ \\
\hline 291.0 & $1.07 \mathrm{E}+01$ & $2.96 \mathrm{E}+00$ & $\mathrm{cpt}$ \\
\hline 290.0 & $2.66 \mathrm{E}+01$ & $7.38 \mathrm{E}+00$ & $\mathrm{cpt}$ \\
\hline 289.0 & $2.13 \mathrm{E}+01$ & $5.91 \mathrm{E}+00$ & $\mathrm{cpt}$ \\
\hline 288.0 & $1.47 \mathrm{E}-03$ & $4.10 \mathrm{E}-04$ & $\mathrm{cpt}$ \\
\hline 287.0 & $1.25 \mathrm{E}-03$ & $3.50 \mathrm{E}-04$ & $\mathrm{cpt}$ \\
\hline 286.0 & $1.19 \mathrm{E}+01$ & $3.31 \mathrm{E}+00$ & $\mathrm{cpt}$ \\
\hline 285.0 & $2.18 \mathrm{E}+00$ & $6.05 \mathrm{E}-01$ & $\mathrm{cpt}$ \\
\hline 284.0 & $5.03 \mathrm{E}-01$ & $1.40 \mathrm{E}-01$ & $\mathrm{cpt}$ \\
\hline 283.0 & $7.40 \mathrm{E}+01$ & $2.06 \mathrm{E}+01$ & $\mathrm{cpt}$ \\
\hline 282.0 & $1.00 \mathrm{E}+02$ & $2.78 \mathrm{E}+01$ & $\mathrm{cpt}$ \\
\hline 281.0 & $7.31 \mathrm{E}+00$ & $2.03 \mathrm{E}+00$ & $\mathrm{cpt}$ \\
\hline 280.0 & $5.63 \mathrm{E}+00$ & $1.57 \mathrm{E}+00$ & $\mathrm{cpt}$ \\
\hline 279.0 & $3.58 \mathrm{E}-02$ & $9.94 \mathrm{E}-03$ & $\mathrm{cpt}$ \\
\hline 278.0 & $1.33 \mathrm{E}+00$ & $3.68 \mathrm{E}-01$ & $\mathrm{cpt}$ \\
\hline 277.0 & $1.14 \mathrm{E}+01$ & $3.17 \mathrm{E}+00$ & $\mathrm{cpt}$ \\
\hline 276.0 & $7.71 \mathrm{E}+00$ & $2.14 \mathrm{E}+00$ & $\mathrm{cpt}$ \\
\hline 275.0 & $1.46 \mathrm{E}+00$ & $4.05 \mathrm{E}-01$ & $\mathrm{cpt}$ \\
\hline 274.0 & $8.90 \mathrm{E}+00$ & $2.47 \mathrm{E}+00$ & $\mathrm{cpt}$ \\
\hline 273.0 & $1.75 \mathrm{E}+01$ & $4.85 \mathrm{E}+00$ & $\mathrm{cpt}$ \\
\hline 272.0 & $1.44 \mathrm{E}+01$ & $4.00 \mathrm{E}+00$ & $\mathrm{cpt}$ \\
\hline 271.0 & $1.78 \mathrm{E}+01$ & $4.95 \mathrm{E}+00$ & $\mathrm{cpt}$ \\
\hline 270.0 & $8.82 \mathrm{E}+00$ & $2.45 \mathrm{E}+00$ & $\mathrm{cpt}$ \\
\hline 269.0 & $8.08 \mathrm{E}+00$ & $2.25 \mathrm{E}+00$ & $\mathrm{cpt}$ \\
\hline 268.0 & $1.00 \mathrm{E}+02$ & $2.78 \mathrm{E}+01$ & $\mathrm{cpt}$ \\
\hline 267.0 & $1.00 \mathrm{E}+02$ & $2.78 \mathrm{E}+01$ & $\mathrm{cpt}$ \\
\hline 266.0 & $2.42 \mathrm{E}+00$ & $6.73 \mathrm{E}-01$ & $\mathrm{cpt}$ \\
\hline 265.0 & $1.00 \mathrm{E}+02$ & $2.78 \mathrm{E}+01$ & $\mathrm{cpt}$ \\
\hline 264.0 & $1.85 \mathrm{E}-01$ & $5.13 \mathrm{E}-02$ & $\mathrm{cpt}$ \\
\hline 263.0 & $1.66 \mathrm{E}-01$ & $4.61 \mathrm{E}-02$ & $\mathrm{cpt}$ \\
\hline 261.0 & $2.26 \mathrm{E}-01$ & $6.29 \mathrm{E}-02$ & $\mathrm{cpt}$ \\
\hline & $8.85 \mathrm{E}-03$ & $2.46 \mathrm{E}-03$ & $\mathrm{cpt}$ \\
\hline
\end{tabular}


Conductivity Data at RPT7

\begin{tabular}{|c|c|c|c|}
\hline $\begin{array}{c}\text { Elevation } \\
\text { (ft) }\end{array}$ & $\begin{array}{c}\mathrm{Kh} \\
\text { (ft/day) }\end{array}$ & $\begin{array}{c}\mathrm{Kv} \\
\text { (ft/day) }\end{array}$ & $\begin{array}{c}\text { Data } \\
\text { Source }\end{array}$ \\
\hline 260.0 & $1.67 \mathrm{E}-02$ & $4.65 E-03$ & $\mathrm{cpt}$ \\
\hline 259.0 & $1.30 \mathrm{E}-03$ & $3.60 \mathrm{E}-04$ & $\mathrm{cpt}$ \\
\hline 258.0 & $4.21 E-03$ & 1.17E-03 & $\mathrm{cpt}$ \\
\hline 257.0 & $1.61 \mathrm{E}-03$ & $4.50 \mathrm{E}-04$ & $\mathrm{cpt}$ \\
\hline 256.0 & $1.25 \mathrm{E}-03$ & $3.50 \mathrm{E}-04$ & cpt \\
\hline 255.0 & $1.18 \mathrm{E}-03$ & $3.30 \mathrm{E}-04$ & cpt \\
\hline 254.0 & $9.30 \mathrm{E}-04$ & $2.60 \mathrm{E}-04$ & cpt \\
\hline 253.0 & $9.40 \mathrm{E}-04$ & $2.60 \mathrm{E}-04$ & $\mathrm{cpt}$ \\
\hline 252.0 & $8.00 E-04$ & $2.20 \mathrm{E}-04$ & $\mathrm{cpt}$ \\
\hline 251.0 & $8.50 E-04$ & $2.40 \mathrm{E}-04$ & $\mathrm{cpt}$ \\
\hline 250.0 & $9.10 \mathrm{E}-04$ & $2.50 \mathrm{E}-04$ & $\mathrm{cpt}$ \\
\hline 249.0 & $1.08 \mathrm{E}-03$ & $3.00 E-04$ & $\mathrm{cpt}$ \\
\hline 248.0 & $7.40 \mathrm{E}-04$ & $2.10 \mathrm{E}-04$ & $\mathrm{cpt}$ \\
\hline 247.0 & $7.40 \mathrm{E}-04$ & $2.10 \mathrm{E}-04$ & $\mathrm{cpt}$ \\
\hline 246.0 & $6.80 \mathrm{E}-04$ & $1.90 \mathrm{E}-04$ & $\mathrm{cpt}$ \\
\hline 245.0 & $8.00 \mathrm{E}-04$ & $2.20 \mathrm{E}-04$ & $\mathrm{cpt}$ \\
\hline 244.0 & $6.76 \mathrm{E}+00$ & $1.88 \mathrm{E}+00$ & cpt \\
\hline 243.0 & $8.68 \mathrm{E}-02$ & $2.41 \mathrm{E}-02$ & cpt \\
\hline 242.0 & $8.90 \mathrm{E}-04$ & $2.50 E-04$ & cpt \\
\hline 241.0 & $1.49 \mathrm{E}-03$ & 4.10E-04 & cpt \\
\hline 240.0 & $7.87 \mathrm{E}+00$ & $2.19 E+00$ & $\mathrm{cpt}$ \\
\hline 239.0 & $4.33 E-01$ & $1.20 \mathrm{E}-01$ & cpt \\
\hline 238.0 & $1.07 \mathrm{E}-03$ & $3.00 \mathrm{E}-04$ & cpt \\
\hline 237.0 & $9.06 \mathrm{E}+00$ & $2.52 E+00$ & cpt \\
\hline 236.0 & $1.30 E+01$ & $3.62 \mathrm{E}+00$ & cpt \\
\hline 235.0 & $3.88 E+00$ & $1.08 \mathrm{E}+00$ & cpt \\
\hline 234.0 & $5.08 E-01$ & $1.41 \mathrm{E}-01$ & cpt \\
\hline 233.0 & $8.48 E+00$ & $2.36 \mathrm{E}+00$ & $\mathrm{cpt}$ \\
\hline 232.0 & $1.14 \mathrm{E}+00$ & $3.15 \mathrm{E}-01$ & cpt \\
\hline 231.0 & $3.02 E-03$ & $8.40 E-04$ & $\mathrm{cpt}$ \\
\hline 230.0 & $2.02 E-01$ & $5.61 \mathrm{E}-02$ & $\mathrm{cpt}$ \\
\hline 229.0 & $5.63 E+00$ & $1.56 \mathrm{E}+00$ & cpt \\
\hline 228.0 & $1.91 \mathrm{E}+01$ & $5.29 \mathrm{E}+00$ & $\mathrm{cpt}$ \\
\hline 227.0 & $1.75 \mathrm{E}+01$ & $4.86 \mathrm{E}+00$ & cpt \\
\hline 226.0 & $1.31 E+00$ & $3.63 E-01$ & cpt \\
\hline 225.0 & $2.13 E+00$ & $5.92 \mathrm{E}-01$ & $\mathrm{cpt}$ \\
\hline 224.0 & $1.55 \mathrm{E}-01$ & $4.31 E-02$ & $\mathrm{cpt}$ \\
\hline 223.0 & $9.43 \mathrm{E}-02$ & $2.62 E-02$ & $\mathrm{cpt}$ \\
\hline 222.0 & $4.61 E-01$ & $1.28 \mathrm{E}-01$ & $c p t$ \\
\hline 221.0 & $5.88 E+00$ & $1.63 \mathrm{E}+00$ & $\mathrm{cpt}$ \\
\hline 220.0 & 3.69E-01 & $1.03 E-01$ & $c p t$ \\
\hline 219.0 & $4.16 E+00$ & $1.16 \mathrm{E}+00$ & $\mathrm{cpt}$ \\
\hline 218.0 & $1.00 E+01$ & $2.79 \mathrm{E}+00$ & cpt \\
\hline 217.0 & $6.40 \mathrm{E}+00$ & $1.78 \mathrm{E}+00$ & cpt \\
\hline 216.0 & $1.43 E+00$ & $3.98 \mathrm{E}-01$ & $\mathrm{cpt}$ \\
\hline 215.0 & $2.39 E+01$ & $6.63 E+00$ & $\mathrm{cpt}$ \\
\hline 214.0 & $4.52 E+01$ & $1.26 \mathrm{E}+01$ & cpt \\
\hline 213.0 & $5.63 E+01$ & $1.56 \mathrm{E}+01$ & $\mathrm{cpt}$ \\
\hline
\end{tabular}


Conductivity Data at RPT7

\begin{tabular}{|c|c|c|c|}
\hline $\begin{array}{l}\text { Elevation } \\
\text { (ft) }\end{array}$ & $\begin{array}{c}\mathrm{Kh} \\
\text { (ft/day) }\end{array}$ & $\begin{array}{c}\text { Kv } \\
\text { (ft/day) }\end{array}$ & $\begin{array}{c}\text { Data } \\
\text { Source }\end{array}$ \\
\hline 212.0 & $4.58 E+01$ & $1.27 \mathrm{E}+01$ & $\mathrm{cpt}$ \\
\hline 211.0 & $4.60 \mathrm{E}+01$ & $1.28 \mathrm{E}+01$ & $\mathrm{cpt}$ \\
\hline 210.0 & $4.07 \mathrm{E}+01$ & $1.13 E+01$ & cpt \\
\hline 209.0 & $3.62 E+01$ & $1.00 E+01$ & $\mathrm{cpt}$ \\
\hline 208.0 & $3.99 \mathrm{E}+01$ & $1.11 \mathrm{E}+01$ & $\mathrm{cpt}$ \\
\hline 207.0 & $4.29 \mathrm{E}+01$ & $1.19 \mathrm{E}+01$ & $\mathrm{cpt}$ \\
\hline 206.0 & $4.87 E+01$ & $1.35 \mathrm{E}+01$ & $\mathrm{cpt}$ \\
\hline 205.0 & $6.18 E+01$ & $1.72 \mathrm{E}+01$ & cpt \\
\hline 204.0 & $5.53 E+01$ & $1.54 \mathrm{E}+01$ & cpt \\
\hline 203.0 & $1.97 \mathrm{E}+00$ & $5.47 E-01$ & $\mathrm{cpt}$ \\
\hline 202.0 & 4.05E-03 & $1.13 \mathrm{E}-03$ & cpt \\
\hline 201.0 & $1.37 \mathrm{E}-02$ & $3.79 E-03$ & $\mathrm{cpt}$ \\
\hline 200.0 & $1.18 \mathrm{E}-02$ & $3.28 \mathrm{E}-03$ & $\mathrm{cpt}$ \\
\hline 199.0 & $6.20 \mathrm{E}+01$ & $1.72 \mathrm{E}+01$ & $\mathrm{cpt}$ \\
\hline 198.0 & $1.00 E+02$ & $2.78 \mathrm{E}+01$ & cpt \\
\hline 197.0 & $1.00 \mathrm{E}+02$ & $2.78 \mathrm{E}+01$ & $\mathrm{cpt}$ \\
\hline 196.0 & $1.00 \mathrm{E}+02$ & $2.78 E+01$ & $\mathrm{cpt}$ \\
\hline 195.0 & $1.00 E+02$ & $2.78 \mathrm{E}+01$ & $\mathrm{cpt}$ \\
\hline 194.0 & $1.00 \mathrm{E}+02$ & $2.78 \mathrm{E}+01$ & $\mathrm{cpt}$ \\
\hline 193.0 & $8.60 E+01$ & $2.39 \mathrm{E}+01$ & $\mathrm{cpt}$ \\
\hline 192.0 & $8.72 E+01$ & $2.42 E+01$ & $\mathrm{cpt}$ \\
\hline 191.0 & $1.00 \mathrm{E}+02$ & $2.78 E+01$ & cpt \\
\hline 190.0 & $1.00 \mathrm{E}+02$ & $2.78 \mathrm{E}+01$ & $\mathrm{cpt}$ \\
\hline 189.0 & $8.67 E+01$ & $2.41 E+01$ & cpt \\
\hline 188.0 & $5.56 \mathrm{E}+01$ & $1.54 \mathrm{E}+01$ & cpt \\
\hline 187.0 & $4.73 \mathrm{E}+01$ & $1.31 \mathrm{E}+01$ & cpt \\
\hline 186.0 & $4.48 \mathrm{E}+01$ & $1.24 \mathrm{E}+01$ & $\mathrm{cpt}$ \\
\hline 185.0 & $4.30 \mathrm{E}+01$ & $1.20 \mathrm{E}+01$ & $\mathrm{cpt}$ \\
\hline 184.0 & $2.92 \mathrm{E}+01$ & $8.11 E+00$ & $\mathrm{cpt}$ \\
\hline 183.0 & $2.60 E+01$ & $7.23 E+00$ & $\mathrm{cpt}$ \\
\hline 182.0 & $3.30 \mathrm{E}+01$ & $9.18 E+00$ & $\mathrm{cpt}$ \\
\hline 181.0 & $3.03 E+01$ & $8.42 E+00$ & cpt \\
\hline 180.0 & $2.97 E+01$ & $8.24 \mathrm{E}+00$ & $\mathrm{cpt}$ \\
\hline 179.0 & $2.61 \mathrm{E}+01$ & $7.24 \mathrm{E}+00$ & $\mathrm{cpt}$ \\
\hline 178.0 & $3.22 E+01$ & $8.96 E+00$ & $\mathrm{cpt}$ \\
\hline 177.0 & $3.79 \mathrm{E}+01$ & $1.05 \mathrm{E}+01$ & $\mathrm{cpt}$ \\
\hline 176.0 & $2.29 E+01$ & $6.36 \mathrm{E}+00$ & $\mathrm{cpt}$ \\
\hline 175.0 & $6.66 \mathrm{E}-02$ & $1.85 \mathrm{E}-02$ & $\mathrm{cpt}$ \\
\hline 174.0 & $1.67 \mathrm{E}-03$ & $4.60 \mathrm{E}-04$ & $\mathrm{cpt}$ \\
\hline 173.0 & $1.92 \mathrm{E}+00$ & $5.33 \mathrm{E}-01$ & $c p t$ \\
\hline 172.0 & $1.37 \mathrm{E}-03$ & $3.80 \mathrm{E}-04$ & cpt \\
\hline 171.0 & $1.72 \mathrm{E}-03$ & $4.80 \mathrm{E}-04$ & cpt \\
\hline 170.0 & $7.70 \mathrm{E}-04$ & $2.20 \mathrm{E}-04$ & cpt \\
\hline 169.0 & $6.88 \mathrm{E}-01$ & $1.91 \mathrm{E}-01$ & cpt \\
\hline 168.0 & $2.76 \mathrm{E}+01$ & $7.66 \mathrm{E}+00$ & $\mathrm{cpt}$ \\
\hline 167.0 & $2.63 E+01$ & $7.30 \mathrm{E}+00$ & cpt \\
\hline 166.0 & $2.81 E+01$ & $7.80 \mathrm{E}+00$ & $\mathrm{cpt}$ \\
\hline 165.0 & $2.86 E+01$ & $7.95 \mathrm{E}+00$ & $\mathrm{cpt}$ \\
\hline
\end{tabular}


Conductivity Data at RPT7

\begin{tabular}{|c|c|c|c|}
\hline $\begin{array}{c}\text { Elevation } \\
\text { (ft) }\end{array}$ & $\begin{array}{c}\text { Kh } \\
\text { (ft/day) }\end{array}$ & $\begin{array}{c}\text { Kv } \\
\text { (ft/day) }\end{array}$ & $\begin{array}{c}\text { Data } \\
\text { Source }\end{array}$ \\
\hline 164.0 & $3.01 \mathrm{E}+01$ & $8.37 \mathrm{E}+00$ & $\mathrm{cpt}$ \\
\hline 163.0 & $2.65 \mathrm{E}-01$ & $7.37 \mathrm{E}-02$ & $\mathrm{cpt}$ \\
\hline 162.0 & $5.05 \mathrm{E}-01$ & $1.40 \mathrm{E}-01$ & $\mathrm{cpt}$ \\
\hline 161.0 & $1.68 \mathrm{E}-03$ & $4.70 \mathrm{E}-04$ & $\mathrm{cpt}$ \\
\hline 160.0 & $1.24 \mathrm{E}-03$ & $3.50 \mathrm{E}-04$ & $\mathrm{cpt}$ \\
\hline 159.0 & $3.17 \mathrm{E}-03$ & $8.80 \mathrm{E}-04$ & $\mathrm{cpt}$ \\
\hline 158.0 & $2.15 \mathrm{E}-03$ & $6.00 \mathrm{E}-04$ & $\mathrm{cpt}$ \\
\hline 157.0 & $2.59 \mathrm{E}+00$ & $7.19 \mathrm{E}-01$ & $\mathrm{cpt}$ \\
\hline 156.0 & $4.36 \mathrm{E}+01$ & $1.21 \mathrm{E}+01$ & $\mathrm{cpt}$ \\
\hline 155.0 & $3.96 \mathrm{E}+01$ & $1.10 \mathrm{E}+01$ & $\mathrm{cpt}$ \\
\hline 154.0 & $6.64 \mathrm{E}-03$ & $1.84 \mathrm{E}-03$ & $\mathrm{cpt}$ \\
\hline 153.0 & $2.45 \mathrm{E}-02$ & $6.81 \mathrm{E}-03$ & $\mathrm{cpt}$ \\
\hline 152.0 & $7.87 \mathrm{E}+01$ & $2.19 \mathrm{E}+01$ & $\mathrm{cpt}$ \\
\hline 151.0 & $3.78 \mathrm{E}+01$ & $1.05 \mathrm{E}+01$ & $\mathrm{cpt}$ \\
\hline 150.0 & $4.49 \mathrm{E}+01$ & $1.25 \mathrm{E}+01$ & $\mathrm{cpt}$ \\
\hline 149.0 & $1.00 \mathrm{E}+02$ & $2.78 \mathrm{E}+01$ & $\mathrm{cpt}$ \\
\hline 148.0 & $1.00 \mathrm{E}+02$ & $2.78 \mathrm{E}+01$ & $\mathrm{cpt}$ \\
\hline 147.0 & $4.40 \mathrm{E}+01$ & $1.22 \mathrm{E}+01$ & $\mathrm{cpt}$ \\
\hline 146.0 & $2.94 \mathrm{E}+01$ & $8.15 \mathrm{E}+00$ & $\mathrm{cpt}$ \\
\hline 145.0 & $6.49 \mathrm{E}+01$ & $1.80 \mathrm{E}+01$ & $\mathrm{cpt}$ \\
\hline 144.0 & $2.69 \mathrm{E}+01$ & $7.47 \mathrm{E}+00$ & $\mathrm{cpt}$ \\
\hline 143.0 & $7.31 \mathrm{E}+01$ & $2.03 \mathrm{E}+01$ & $\mathrm{cpt}$ \\
\hline 142.0 & $1.00 \mathrm{E}+02$ & $2.78 \mathrm{E}+01$ & $\mathrm{cpt}$ \\
\hline 141.0 & $1.00 \mathrm{E}+02$ & $2.78 \mathrm{E}+01$ & $\mathrm{cpt}$ \\
\hline 140.0 & $6.38 \mathrm{E}+01$ & $1.77 \mathrm{E}+01$ & $\mathrm{cpt}$ \\
\hline 139.0 & $4.41 \mathrm{E}+01$ & $1.22 \mathrm{E}+01$ & $\mathrm{cpt}$ \\
\hline 138.0 & $1.00 \mathrm{E}+02$ & $2.78 \mathrm{E}+01$ & $\mathrm{cpt}$ \\
\hline 137.0 & $1.00 \mathrm{E}+02$ & $2.78 \mathrm{E}+01$ & $\mathrm{cpt}$ \\
\hline 136.0 & $1.00 \mathrm{E}+02$ & $2.78 \mathrm{E}+01$ & $\mathrm{cpt}$ \\
\hline 135.0 & $6.88 \mathrm{E}+01$ & $1.91 \mathrm{E}+01$ & $\mathrm{cpt}$ \\
\hline 134.0 & $1.00 \mathrm{E}+02$ & $2.78 \mathrm{E}+01$ & $\mathrm{cpt}$ \\
\hline 133.0 & $1.00 \mathrm{E}+02$ & $2.78 \mathrm{E}+01$ & $\mathrm{cpt}$ \\
\hline 132.0 & $7.58 \mathrm{E}+01$ & $2.11 \mathrm{E}+01$ & $\mathrm{cpt}$ \\
\hline 131.0 & $2.35 \mathrm{E}+01$ & $6.53 \mathrm{E}+00$ & $\mathrm{cpt}$ \\
\hline 130.0 & $2.47 \mathrm{E}+01$ & $6.85 \mathrm{E}+00$ & $\mathrm{cpt}$ \\
\hline 129.0 & $1.00 \mathrm{E}+02$ & $2.78 \mathrm{E}+01$ & $\mathrm{cpt}$ \\
\hline 128.0 & $1.00 \mathrm{E}+02$ & $2.78 \mathrm{E}+01$ & $\mathrm{cpt}$ \\
\hline 127.0 & $1.00 \mathrm{E}+02$ & $2.78 \mathrm{E}+01$ & $\mathrm{cpt}$ \\
\hline 126.0 & $3.89 \mathrm{E}+01$ & $1.08 \mathrm{E}+01$ & $\mathrm{cpt}$ \\
\hline 125.0 & $6.97 \mathrm{E}+01$ & $1.94 \mathrm{E}+01$ & $\mathrm{cpt}$ \\
\hline 124.0 & $6.90 \mathrm{E}+01$ & $1.92 \mathrm{E}+01$ & $\mathrm{cpt}$ \\
\hline 123.0 & $7.08 \mathrm{E}+01$ & $1.97 \mathrm{E}+01$ & $\mathrm{cpt}$ \\
\hline 122.0 & $1.00 \mathrm{E}+02$ & $2.78 \mathrm{E}+01$ & $\mathrm{cpt}$ \\
\hline 121.0 & $1.00 \mathrm{E}+02$ & $2.78 \mathrm{E}+01$ & $\mathrm{cpt}$ \\
\hline 120.0 & $1.00 \mathrm{E}+02$ & $2.78 \mathrm{E}+01$ & $\mathrm{cpt}$ \\
\hline 119.0 & $1.00 \mathrm{E}+02$ & $2.78 \mathrm{E}+01$ & $\mathrm{cpt}$ \\
\hline 118.0 & $1.00 \mathrm{E}+02$ & $2.78 \mathrm{E}+01$ & $\mathrm{cpt}$ \\
\hline 117.0 & $1.00 \mathrm{E}+02$ & $2.78 \mathrm{E}+01$ & $\mathrm{cpt}$ \\
\hline & & & \\
\hline
\end{tabular}


Conductivity Data at RPT7

\begin{tabular}{|c|c|c|c|}
\hline $\begin{array}{c}\text { Elevation } \\
\text { (ft) }\end{array}$ & $\begin{array}{c}\text { Kh } \\
\text { (ft/day) }\end{array}$ & $\begin{array}{c}\text { Kv } \\
\text { (ft/day) }\end{array}$ & $\begin{array}{c}\text { Data } \\
\text { Source }\end{array}$ \\
\hline 116.0 & $1.00 \mathrm{E}+02$ & $2.78 \mathrm{E}+01$ & $\mathrm{cpt}$ \\
\hline 115.0 & $1.00 \mathrm{E}+02$ & $2.78 \mathrm{E}+01$ & $\mathrm{cpt}$ \\
\hline 114.0 & $1.00 \mathrm{E}+02$ & $2.78 \mathrm{E}+01$ & $\mathrm{cpt}$ \\
\hline 113.0 & $5.86 \mathrm{E}+01$ & $1.63 \mathrm{E}+01$ & $\mathrm{cpt}$ \\
\hline 112.0 & $4.88 \mathrm{E}+01$ & $1.36 \mathrm{E}+01$ & $\mathrm{cpt}$ \\
\hline 111.0 & $8.84 \mathrm{E}+01$ & $2.45 \mathrm{E}+01$ & $\mathrm{cpt}$ \\
\hline 110.0 & $1.00 \mathrm{E}+02$ & $2.78 \mathrm{E}+01$ & $\mathrm{cpt}$ \\
\hline 109.0 & $1.00 \mathrm{E}+02$ & $2.78 \mathrm{E}+01$ & $\mathrm{cpt}$ \\
\hline 108.0 & $1.00 \mathrm{E}+02$ & $2.78 \mathrm{E}+01$ & $\mathrm{cpt}$ \\
\hline 107.0 & $1.00 \mathrm{E}+02$ & $2.78 \mathrm{E}+01$ & $\mathrm{cpt}$ \\
\hline 106.0 & $1.00 \mathrm{E}+02$ & $2.78 \mathrm{E}+01$ & $\mathrm{cpt}$ \\
\hline 105.0 & $1.00 \mathrm{E}+02$ & $2.78 \mathrm{E}+01$ & $\mathrm{cpt}$ \\
\hline 104.0 & $1.00 \mathrm{E}+02$ & $2.78 \mathrm{E}+01$ & $\mathrm{cpt}$ \\
\hline 103.0 & $1.00 \mathrm{E}+02$ & $2.78 \mathrm{E}+01$ & $\mathrm{cpt}$ \\
\hline 102.0 & $1.00 \mathrm{E}+02$ & $2.78 \mathrm{E}+01$ & $\mathrm{cpt}$ \\
\hline 101.0 & $1.00 \mathrm{E}+02$ & $2.78 \mathrm{E}+01$ & $\mathrm{cpt}$ \\
\hline 100.0 & $5.10 \mathrm{E}+01$ & $1.42 \mathrm{E}+01$ & $\mathrm{cpt}$ \\
\hline
\end{tabular}


Conductivity Data at RPT8

\begin{tabular}{|c|c|c|c|}
\hline $\begin{array}{c}\text { Elevation } \\
\text { (ft) }\end{array}$ & $\begin{array}{c}\text { Kh } \\
\text { (ft/day) }\end{array}$ & $\begin{array}{c}\text { Kv } \\
\text { (ft/day) }\end{array}$ & $\begin{array}{c}\text { Data } \\
\text { Source }\end{array}$ \\
\hline 307.4 & $1.00 \mathrm{E}+01$ & $2.78 \mathrm{E}+00$ & $\mathrm{cpt}$ \\
\hline 306.4 & $3.26 \mathrm{E}+01$ & $9.06 \mathrm{E}+00$ & $\mathrm{cpt}$ \\
\hline 305.4 & $1.56 \mathrm{E}+01$ & $4.34 \mathrm{E}+00$ & $\mathrm{cpt}$ \\
\hline 304.4 & $6.22 \mathrm{E}+00$ & $1.73 \mathrm{E}+00$ & $\mathrm{cpt}$ \\
\hline 303.4 & $6.28 \mathrm{E}+00$ & $1.74 \mathrm{E}+00$ & $\mathrm{cpt}$ \\
\hline 302.4 & $8.72 \mathrm{E}+00$ & $2.42 \mathrm{E}+00$ & $\mathrm{cpt}$ \\
\hline 301.4 & $6.55 \mathrm{E}+00$ & $1.82 \mathrm{E}+00$ & $\mathrm{cpt}$ \\
\hline 300.4 & $1.24 \mathrm{E}-02$ & $3.45 \mathrm{E}-03$ & $\mathrm{cpt}$ \\
\hline 299.4 & $2.59 \mathrm{E}-03$ & $7.20 \mathrm{E}-04$ & $\mathrm{cpt}$ \\
\hline 298.4 & $7.50 \mathrm{E}-01$ & $2.08 \mathrm{E}-01$ & $\mathrm{cpt}$ \\
\hline 297.4 & $1.62 \mathrm{E}-01$ & $4.50 \mathrm{E}-02$ & $\mathrm{cpt}$ \\
\hline 296.4 & $2.79 \mathrm{E}-03$ & $7.70 \mathrm{E}-04$ & $\mathrm{cpt}$ \\
\hline 295.4 & $1.45 \mathrm{E}-03$ & $4.00 \mathrm{E}-04$ & $\mathrm{cpt}$ \\
\hline 294.4 & $7.92 \mathrm{E}+00$ & $2.20 \mathrm{E}+00$ & $\mathrm{cpt}$ \\
\hline 293.4 & $3.70 \mathrm{E}-02$ & $1.03 \mathrm{E}-02$ & $\mathrm{cpt}$ \\
\hline 292.4 & $1.70 \mathrm{E}-03$ & $4.70 \mathrm{E}-04$ & $\mathrm{cpt}$ \\
\hline 291.4 & $2.53 \mathrm{E}-03$ & $7.00 \mathrm{E}-04$ & $\mathrm{cpt}$ \\
\hline 290.4 & $2.52 \mathrm{E}-03$ & $7.00 \mathrm{E}-04$ & $\mathrm{cpt}$ \\
\hline 289.4 & $1.47 \mathrm{E}-03$ & $4.10 \mathrm{E}-04$ & $\mathrm{cpt}$ \\
\hline 288.4 & $1.24 \mathrm{E}-02$ & $3.44 \mathrm{E}-03$ & $\mathrm{cpt}$ \\
\hline 287.4 & $1.96 \mathrm{E}-03$ & $5.40 \mathrm{E}-04$ & $\mathrm{cpt}$ \\
\hline 286.4 & $2.99 \mathrm{E}-03$ & $8.30 \mathrm{E}-04$ & $\mathrm{cpt}$ \\
\hline 285.4 & $2.32 \mathrm{E}-03$ & $6.40 \mathrm{E}-04$ & $\mathrm{cpt}$ \\
\hline 284.4 & $3.22 \mathrm{E}-03$ & $8.90 \mathrm{E}-04$ & $\mathrm{cpt}$ \\
\hline 283.4 & $1.03 \mathrm{E}-03$ & $2.90 \mathrm{E}-04$ & $\mathrm{cpt}$ \\
\hline 282.4 & $9.50 \mathrm{E}-04$ & $2.60 \mathrm{E}-04$ & $\mathrm{cpt}$ \\
\hline 281.4 & $8.80 \mathrm{E}-04$ & $2.40 \mathrm{E}-04$ & $\mathrm{cpt}$ \\
\hline 280.4 & $6.60 \mathrm{E}-04$ & $1.80 \mathrm{E}-04$ & $\mathrm{cpt}$ \\
\hline 279.4 & $7.27 \mathrm{E}+00$ & $2.02 \mathrm{E}+00$ & $\mathrm{cpt}$ \\
\hline 278.4 & $4.94 \mathrm{E}-01$ & $1.37 \mathrm{E}-01$ & $\mathrm{cpt}$ \\
\hline 277.4 & $2.15 \mathrm{E}+00$ & $5.97 \mathrm{E}-01$ & $\mathrm{cpt}$ \\
\hline 276.4 & $4.11 \mathrm{E}-01$ & $1.14 \mathrm{E}-01$ & $\mathrm{cpt}$ \\
\hline 275.4 & $1.26 \mathrm{E}-03$ & $3.50 \mathrm{E}-04$ & $\mathrm{cpt}$ \\
\hline 274.4 & $1.10 \mathrm{E}-03$ & $3.00 \mathrm{E}-04$ & $\mathrm{cpt}$ \\
\hline 273.4 & $1.05 \mathrm{E}-03$ & $2.90 \mathrm{E}-04$ & $\mathrm{cpt}$ \\
\hline 272.4 & $8.30 \mathrm{E}-04$ & $2.30 \mathrm{E}-04$ & $\mathrm{cpt}$ \\
\hline 271.4 & $7.80 \mathrm{E}-04$ & $2.20 \mathrm{E}-04$ & $\mathrm{cpt}$ \\
\hline 270.4 & $7.40 \mathrm{E}-04$ & $2.10 \mathrm{E}-04$ & $\mathrm{cpt}$ \\
\hline 269.4 & $7.50 \mathrm{E}-04$ & $2.10 \mathrm{E}-04$ & $\mathrm{cpt}$ \\
\hline 268.4 & $5.60 \mathrm{E}-04$ & $1.60 \mathrm{E}-04$ & $\mathrm{cpt}$ \\
\hline 267.4 & $4.60 \mathrm{E}-04$ & $1.30 \mathrm{E}-04$ & $\mathrm{cpt}$ \\
\hline 266.4 & $5.10 \mathrm{E}-04$ & $1.40 \mathrm{E}-04$ & $\mathrm{cpt}$ \\
\hline 265.4 & $5.00 \mathrm{E}-04$ & $1.40 \mathrm{E}-04$ & $\mathrm{cpt}$ \\
\hline 264.4 & $7.20 \mathrm{E}-04$ & $2.00 \mathrm{E}-04$ & $\mathrm{cpt}$ \\
\hline 263.4 & $8.50 \mathrm{E}-04$ & $2.40 \mathrm{E}-04$ & $\mathrm{cpt}$ \\
\hline 262.4 & $2.94 \mathrm{E}-03$ & $8.20 \mathrm{E}-04$ & $\mathrm{cpt}$ \\
\hline 261.4 & $7.70 \mathrm{E}-04$ & $2.10 \mathrm{E}-04$ & $\mathrm{cpt}$ \\
\hline 260.4 & $5.90 \mathrm{E}-04$ & $1.60 \mathrm{E}-04$ & $\mathrm{cpt}$ \\
\hline & & & \\
\hline
\end{tabular}


Conductivity Data at RPT8

\begin{tabular}{|c|c|c|c|}
\hline $\begin{array}{c}\text { Elevation } \\
\text { (ft) }\end{array}$ & $\begin{array}{c}\text { Kh } \\
\text { (ft/day) }\end{array}$ & $\begin{array}{c}\mathrm{Kv} \\
\text { (ft/day) }\end{array}$ & $\begin{array}{c}\text { Data } \\
\text { Source }\end{array}$ \\
\hline 259.4 & $7.40 \mathrm{E}-04$ & $2.10 \mathrm{E}-04$ & $\overline{c p t}$ \\
\hline 258.4 & $4.70 \mathrm{E}-04$ & $1.30 \mathrm{E}-04$ & $\mathrm{cpt}$ \\
\hline 257.4 & $4.90 \mathrm{E}-04$ & $1.40 \mathrm{E}-04$ & $\mathrm{cpt}$ \\
\hline 256.4 & $4.90 \mathrm{E}-04$ & $1.40 \mathrm{E}-04$ & $\mathrm{cpt}$ \\
\hline 255.4 & $6.41 \mathrm{E}-03$ & $1.78 \mathrm{E}-03$ & $\mathrm{cpt}$ \\
\hline 254.4 & $5.50 \mathrm{E}-04$ & $1.50 \mathrm{E}-04$ & $\mathrm{cpt}$ \\
\hline 253.4 & $1.01 \mathrm{E}-03$ & $2.80 \mathrm{E}-04$ & $\mathrm{cpt}$ \\
\hline 252.4 & $1.11 \mathrm{E}-03$ & $3.10 E-04$ & cpt \\
\hline 251.4 & $6.30 \mathrm{E}+00$ & $1.75 \mathrm{E}+00$ & $\mathrm{cpt}$ \\
\hline 250.4 & $5.05 E-03$ & $1.40 \mathrm{E}-03$ & $\mathrm{cpt}$ \\
\hline 249.4 & $4.92 \mathrm{E}-03$ & 1.37E-03 & $\mathrm{cpt}$ \\
\hline 248.4 & $6.01 \mathrm{E}+00$ & $1.67 E+00$ & cpt \\
\hline 247.4 & $6.62 E+00$ & $1.84 \mathrm{E}+00$ & cpt \\
\hline 246.4 & $9.49 \mathrm{E}-01$ & $2.64 \mathrm{E}-01$ & cpt \\
\hline 245.4 & $2.50 \mathrm{E}-02$ & $6.94 \mathrm{E}-03$ & cpt \\
\hline 244.4 & $2.76 E-02$ & $7.66 \mathrm{E}-03$ & $\mathrm{cpt}$ \\
\hline 243.4 & $7.12 E+00$ & $1.98 \mathrm{E}+00$ & $\mathrm{cpt}$ \\
\hline 242.4 & $7.22 \mathrm{E}+00$ & $2.01 E+00$ & $\mathrm{cpt}$ \\
\hline 241.4 & $8.60 E+00$ & $2.39 E+00$ & cpt \\
\hline 240.4 & $1.00 \mathrm{E}+01$ & $2.79 \mathrm{E}+00$ & cpt \\
\hline 239.4 & $1.20 E+01$ & $3.34 \mathrm{E}+00$ & cpt \\
\hline 238.4 & $1.37 E+01$ & $3.80 E+00$ & cpt \\
\hline 237.4 & $1.41 \mathrm{E}+01$ & $3.91 E+00$ & cpt \\
\hline 236.4 & $3.79 E-03$ & $1.05 \mathrm{E}-03$ & $\mathrm{cpt}$ \\
\hline 235.4 & $9.90 \mathrm{E}-04$ & $2.80 E-04$ & $\mathrm{cpt}$ \\
\hline 234.4 & $3.36 \mathrm{E}+00$ & $9.33 \mathrm{E}-01$ & cpt \\
\hline 233.4 & $7.02 E+00$ & $1.95 E+00$ & cpt \\
\hline 232.4 & $7.00 \mathrm{E}+00$ & $1.95 E+00$ & $\mathrm{cpt}$ \\
\hline 231.4 & $8.04 \mathrm{E}+00$ & $2.23 \mathrm{E}+00$ & cpt \\
\hline 230.4 & $8.30 \mathrm{E}-04$ & $2.30 \mathrm{E}-04$ & $\mathrm{cpt}$ \\
\hline 229.4 & $6.60 \mathrm{E}-04$ & $1.80 \mathrm{E}-04$ & $\mathrm{cpt}$ \\
\hline 228.4 & $1.24 \mathrm{E}-03$ & $3.40 \mathrm{E}-04$ & cpt \\
\hline 227.4 & $3.18 \mathrm{E}-03$ & $8.80 \mathrm{E}-04$ & cpt \\
\hline 226.4 & $2.65 \mathrm{E}+00$ & $7.35 \mathrm{E}-01$ & $\mathrm{cpt}$ \\
\hline 225.4 & $1.72 E+01$ & $4.77 E+00$ & cpt \\
\hline 224.4 & $2.38 E+01$ & $6.62 E+00$ & $\mathrm{cpt}$ \\
\hline 223.4 & $1.46 \mathrm{E}+01$ & $4.05 \mathrm{E}+00$ & cpt \\
\hline 222.4 & $9.06 \mathrm{E}+00$ & $2.52 E+00$ & cpt \\
\hline 221.4 & $4.25 E-03$ & $1.18 \mathrm{E}-03$ & $\mathrm{cpt}$ \\
\hline 220.4 & $1.56 \mathrm{E}-02$ & $4.34 \mathrm{E}-03$ & $\mathrm{cpt}$ \\
\hline 219.4 & $2.04 \mathrm{E}-03$ & $5.70 \mathrm{E}-04$ & $\mathrm{cpt}$ \\
\hline 218.4 & $6.25 \mathrm{E}-01$ & $1.74 \mathrm{E}-01$ & cpt \\
\hline 217.4 & $7.22 \mathrm{E}-03$ & $2.00 \mathrm{E}-03$ & $\mathrm{cpt}$ \\
\hline 216.4 & $1.81 \mathrm{E}+01$ & $5.02 \mathrm{E}+00$ & $\mathrm{cpt}$ \\
\hline 215.4 & $2.18 E+01$ & $6.06 \mathrm{E}+00$ & $\mathrm{cpt}$ \\
\hline 214.4 & $1.41 \mathrm{E}+01$ & $3.91 E+00$ & cpt \\
\hline 213.4 & $2.23 E+01$ & $6.18 \mathrm{E}+00$ & $\mathrm{cpt}$ \\
\hline 212.4 & $1.19 E+01$ & $3.31 E+00$ & $\mathrm{cpt}$ \\
\hline
\end{tabular}




\section{Conductivity Data at RPT8}

\begin{tabular}{|c|c|c|c|}
\hline $\begin{array}{c}\text { Elevation } \\
\text { (ft) }\end{array}$ & $\begin{array}{c}\text { Kh } \\
\text { (ft/day) }\end{array}$ & $\begin{array}{c}\mathrm{Kv} \\
\text { (ft/day) }\end{array}$ & $\begin{array}{c}\text { Data } \\
\text { Source }\end{array}$ \\
\hline 211.4 & $1.80 \mathrm{E}+01$ & $4.99 E+00$ & cpt \\
\hline 210.4 & $1.29 \mathrm{E}+01$ & $3.59 \mathrm{E}+00$ & $\mathrm{cpt}$ \\
\hline 209.4 & $1.99 E+01$ & $5.54 \mathrm{E}+00$ & cpt \\
\hline 208.4 & $2.40 \mathrm{E}+01$ & $6.66 \mathrm{E}+00$ & cpt \\
\hline 207.4 & $2.09 E \div 01$ & $5.80 \mathrm{E}+00$ & cpt \\
\hline 206.4 & $7.57 \mathrm{E}-03$ & $2.10 \mathrm{E}-03$ & $\mathrm{cpt}$ \\
\hline 205.4 & $8.90 E-04$ & 2.50 E-04 & cpt \\
\hline 204.4 & $4.00 \mathrm{E}-03$ & $1.11 \mathrm{E}-03$ & $\mathrm{cpt}$ \\
\hline 203.4 & $4.63 \mathrm{E}+00$ & $1.29 \mathrm{E}+00$ & cpt \\
\hline 202.4 & $3.55 \mathrm{E}-03$ & $9.90 \mathrm{E}-04$ & cpt \\
\hline 201.4 & 4.69E-01 & $1.30 \mathrm{E}-01$ & cpt \\
\hline 200.4 & $3.72 \mathrm{E}+00$ & $1.04 \mathrm{E}+00$ & $\mathrm{cpt}$ \\
\hline 199.4 & $1.04 \mathrm{E}+01$ & $2.88 \mathrm{E}+00$ & cpt \\
\hline 198.4 & $1.83 \mathrm{E}+01$ & $5.09 \mathrm{E}+00$ & $\mathrm{cpt}$ \\
\hline 197.4 & $6.38 \mathrm{E}+00$ & $1.77 \mathrm{E}+00$ & cpt \\
\hline 196.4 & $2.98 E+00$ & $8.29 E-01$ & $\mathrm{cpt}$ \\
\hline$\overline{195.4}$ & $1.67 \mathrm{E}+00$ & $4.64 E-01$ & cpt \\
\hline 194.4 & $2.00 \mathrm{E}+00$ & $5.57 \mathrm{E}-01$ & $\mathrm{cpt}$ \\
\hline 193.4 & $5.49 \mathrm{E}+00$ & $1.53 \mathrm{E}+00$ & cpt \\
\hline 192.4 & $8.89 \mathrm{E}+00$ & $2.47 E+00$ & $\mathrm{cpt}$ \\
\hline 191.4 & $3.62 \mathrm{E}+01$ & $1.01 E+01$ & cpt \\
\hline 190.4 & $3.49 \mathrm{E}+01$ & $9.71 \mathrm{E}+00$ & cpt \\
\hline 189.4 & $3.49 \mathrm{E}+01$ & $9.68 \mathrm{E}+00$ & $\mathrm{cpt}$ \\
\hline 188.4 & $2.73 \mathrm{E}+01$ & $7.57 \mathrm{E}+00$ & $\mathrm{cpt}$ \\
\hline 187.4 & $1.63 \mathrm{E}+01$ & $4.52 \mathrm{E}+00$ & cpt \\
\hline 186.4 & $2.81 \mathrm{E}+01$ & $7.81 \mathrm{E}+00$ & $\mathrm{cpt}$ \\
\hline 185.4 & $3.17 E+01$ & $8.80 \mathrm{E}+00$ & cpt \\
\hline 184.4 & $2.98 E+01$ & $8.29 E+00$ & cpt \\
\hline 183.4 & $2.93 E+01$ & $8.14 E+00$ & cpt \\
\hline 182.4 & $3.27 E+01$ & $9.07 \mathrm{E}+00$ & $\mathrm{cpt}$ \\
\hline 181.4 & $4.93 \mathrm{E}+01$ & 1.37E+01 & $\mathrm{cpt}$ \\
\hline 180.4 & $3.90 E+01$ & $1.08 \mathrm{E}+01$ & cpt \\
\hline 179.4 & $3.24 \mathrm{E}+01$ & $9.01 E+00$ & $\mathrm{cpt}$ \\
\hline 178.4 & $3.07 \mathrm{E}+01$ & $8.53 \mathrm{E}+00$ & $\mathrm{cpt}$ \\
\hline 177.4 & 1.19E-02 & $3.29 E-03$ & $\mathrm{cpt}$ \\
\hline 176.4 & $2.06 \mathrm{E}-03$ & $5.70 \mathrm{E}-04$ & $\mathrm{cpt}$ \\
\hline 175.4 & $4.06 \mathrm{E}-02$ & 1.13E-02 & $\mathrm{cpt}$ \\
\hline 174.4 & $1.69 \mathrm{E}-02$ & 4.71E-03 & $\mathrm{cpt}$ \\
\hline 173.4 & $8.53 \mathrm{E}+00$ & $2.37 E+00$ & $\mathrm{cpt}$ \\
\hline 172.4 & $1.64 \mathrm{E}+01$ & $4.57 E+00$ & $\mathrm{cpt}$ \\
\hline 171.4 & $4.45 \mathrm{E}+01$ & $1.24 \mathrm{E}+01$ & $c p t$ \\
\hline 170.4 & $5.13 \mathrm{E}+01$ & $1.42 \mathrm{E}+01$ & cpt \\
\hline 169.4 & $4.84 \mathrm{E}-02$ & $1.34 \mathrm{E}-02$ & $\mathrm{cpt}$ \\
\hline 168.4 & $1.39 E-03$ & $3.90 \mathrm{E}-04$ & $\mathrm{cpt}$ \\
\hline 167.4 & $2.27 \mathrm{E}-01$ & $6.31 \mathrm{E}-02$ & $\mathrm{cpt}$ \\
\hline 166.4 & $2.04 \mathrm{E}-03$ & $5.70 E-04$ & cpt \\
\hline 165.4 & 3.44E-03 & $9.60 \mathrm{E}-04$ & cpt \\
\hline 164.4 & $2.06 \mathrm{E}+00$ & $5.73 \mathrm{E}-01$ & $\mathrm{cpt}$ \\
\hline
\end{tabular}


Conductivity Data at RPT8

\begin{tabular}{|c|c|c|c|}
\hline $\begin{array}{c}\text { Elevation } \\
\text { (ft) }\end{array}$ & $\begin{array}{c}\text { Kh } \\
\text { (ft/day) }\end{array}$ & $\begin{array}{c}\text { Kv } \\
\text { (ft/day) }\end{array}$ & $\begin{array}{c}\text { Data } \\
\text { Source }\end{array}$ \\
\hline 163.4 & $2.80 \mathrm{E}-01$ & $7.78 \mathrm{E}-02$ & $\mathrm{cpt}$ \\
\hline 162.4 & $5.70 \mathrm{E}-03$ & $1.58 \mathrm{E}-03$ & $\mathrm{cpt}$ \\
\hline 161.4 & $4.78 \mathrm{E}-03$ & $1.33 \mathrm{E}-03$ & $\mathrm{cpt}$ \\
\hline 160.4 & $8.56 \mathrm{E}-01$ & $2.38 \mathrm{E}-01$ & $\mathrm{cpt}$ \\
\hline 159.4 & $6.39 \mathrm{E}-01$ & $1.78 \mathrm{E}-01$ & $\mathrm{cpt}$ \\
\hline 158.4 & $4.91 \mathrm{E}+00$ & $1.37 \mathrm{E}+00$ & $\mathrm{cpt}$ \\
\hline 157.4 & $5.06 \mathrm{E}-01$ & $1.41 \mathrm{E}-01$ & $\mathrm{cpt}$ \\
\hline 156.4 & $2.24 \mathrm{E}-02$ & $6.23 \mathrm{E}-03$ & $\mathrm{cpt}$ \\
\hline 155.4 & $4.88 \mathrm{E}+01$ & $1.36 \mathrm{E}+01$ & $\mathrm{cpt}$ \\
\hline 154.4 & $4.72 \mathrm{E}+01$ & $1.31 \mathrm{E}+01$ & $\mathrm{cpt}$ \\
\hline 153.4 & $4.43 \mathrm{E}+01$ & $1.23 \mathrm{E}+01$ & $\mathrm{cpt}$ \\
\hline 152.4 & $6.68 \mathrm{E}+01$ & $1.86 \mathrm{E}+01$ & $\mathrm{cpt}$ \\
\hline 151.4 & $4.13 \mathrm{E}+01$ & $1.15 \mathrm{E}+01$ & $\mathrm{cpt}$ \\
\hline 150.4 & $1.00 \mathrm{E}+02$ & $2.78 \mathrm{E}+01$ & $\mathrm{cpt}$ \\
\hline 149.4 & $1.00 \mathrm{E}+02$ & $2.78 \mathrm{E}+01$ & $\mathrm{cpt}$ \\
\hline 148.4 & $1.00 \mathrm{E}+02$ & $2.78 \mathrm{E}+01$ & $\mathrm{cpt}$ \\
\hline 147.4 & $1.00 \mathrm{E}+02$ & $2.78 \mathrm{E}+01$ & $\mathrm{cpt}$ \\
\hline 146.4 & $1.00 \mathrm{E}+02$ & $2.78 \mathrm{E}+01$ & $\mathrm{cpt}$ \\
\hline 145.4 & $1.00 \mathrm{E}+02$ & $2.78 \mathrm{E}+01$ & $\mathrm{cpt}$ \\
\hline 144.4 & $1.00 \mathrm{E}+02$ & $2.78 \mathrm{E}+01$ & $\mathrm{cpt}$ \\
\hline 143.4 & $1.00 \mathrm{E}+02$ & $2.78 \mathrm{E}+01$ & $\mathrm{cpt}$ \\
\hline 142.4 & $1.00 \mathrm{E}+02$ & $2.78 \mathrm{E}+01$ & $\mathrm{cpt}$ \\
\hline 141.4 & $1.00 \mathrm{E}+02$ & $2.78 \mathrm{E}+01$ & $\mathrm{cpt}$ \\
\hline 140.4 & $1.00 \mathrm{E}+02$ & $2.78 \mathrm{E}+01$ & $\mathrm{cpt}$ \\
\hline 139.4 & $1.00 \mathrm{E}+02$ & $2.78 \mathrm{E}+01$ & $\mathrm{cpt}$ \\
\hline 138.4 & $8.12 \mathrm{E}+01$ & $2.25 \mathrm{E}+01$ & $\mathrm{cpt}$ \\
\hline 137.4 & $1.00 \mathrm{E}+02$ & $2.78 \mathrm{E}+01$ & $\mathrm{cpt}$ \\
\hline 136.4 & $8.21 \mathrm{E}+01$ & $2.28 \mathrm{E}+01$ & $\mathrm{cpt}$ \\
\hline 135.4 & $1.00 \mathrm{E}+02$ & $2.78 \mathrm{E}+01$ & $\mathrm{cpt}$ \\
\hline 134.4 & $1.00 \mathrm{E}+02$ & $2.78 \mathrm{E}+01$ & $\mathrm{cpt}$ \\
\hline 133.4 & $1.00 \mathrm{E}+02$ & $2.78 \mathrm{E}+01$ & $\mathrm{cpt}$ \\
\hline 132.4 & $1.00 \mathrm{E}+02$ & $2.78 \mathrm{E}+01$ & $\mathrm{cpt}$ \\
\hline 131.4 & $3.90 \mathrm{E}+01$ & $1.09 \mathrm{E}+01$ & $\mathrm{cpt}$ \\
\hline 130.4 & $4.62 \mathrm{E}+01$ & $1.28 \mathrm{E}+01$ & $\mathrm{cpt}$ \\
\hline 129.4 & $7.10 \mathrm{E}+01$ & $1.97 \mathrm{E}+01$ & $\mathrm{cpt}$ \\
\hline 128.4 & $4.16 \mathrm{E}+01$ & $1.15 \mathrm{E}+01$ & $\mathrm{cpt}$ \\
\hline 127.4 & $8.54 \mathrm{E}-01$ & $2.37 \mathrm{E}-01$ & $\mathrm{cpt}$ \\
\hline 126.4 & $3.11 \mathrm{E}+01$ & $8.63 \mathrm{E}+00$ & $\mathrm{cpt}$ \\
\hline 125.4 & $2.38 \mathrm{E}+01$ & $6.60 \mathrm{E}+00$ & $\mathrm{cpt}$ \\
\hline 124.4 & $1.49 \mathrm{E}+01$ & $4.13 \mathrm{E}+00$ & $\mathrm{cpt}$ \\
\hline 123.4 & $2.12 \mathrm{E}+01$ & $5.88 \mathrm{E}+00$ & $\mathrm{cpt}$ \\
\hline 122.4 & $4.12 \mathrm{E}+01$ & $1.14 \mathrm{E}+01$ & $\mathrm{cpt}$ \\
\hline 121.4 & $7.04 \mathrm{E}+01$ & $1.96 \mathrm{E}+01$ & $\mathrm{cpt}$ \\
\hline 120.4 & $4.57 \mathrm{E}+01$ & $1.27 \mathrm{E}+01$ & $\mathrm{cpt}$ \\
\hline 119.4 & $3.03 \mathrm{E}+01$ & $8.42 \mathrm{E}+00$ & $\mathrm{cpt}$ \\
\hline 118.4 & $2.61 \mathrm{E}+01$ & $7.25 \mathrm{E}+00$ & $\mathrm{cpt}$ \\
\hline 117.4 & $2.51 \mathrm{E}+01$ & $6.97 \mathrm{E}+00$ & $\mathrm{cpt}$ \\
\hline 116.4 & $2.13 \mathrm{E}+01$ & $5.92 \mathrm{E}+00$ & $\mathrm{cpt}$ \\
\hline & & & \\
\hline
\end{tabular}


Conductivity Data at RPT8

\begin{tabular}{|c|c|c|c|}
\hline $\begin{array}{c}\text { Elevation } \\
\text { (ft) }\end{array}$ & $\begin{array}{c}\text { Kh } \\
\text { (ft/day) }\end{array}$ & $\begin{array}{c}\text { Kv } \\
\text { (ft/day) }\end{array}$ & $\begin{array}{c}\text { Data } \\
\text { Source }\end{array}$ \\
\hline 115.4 & $2.05 \mathrm{E}+01$ & $5.71 \mathrm{E}+00$ & $\mathrm{cpt}$ \\
\hline 114.4 & $2.01 \mathrm{E}+01$ & $5.59 \mathrm{E}+00$ & $\mathrm{cpt}$ \\
\hline 113.4 & $2.08 \mathrm{E}+01$ & $5.78 \mathrm{E}+00$ & $\mathrm{cpt}$ \\
\hline 112.4 & $2.24 \mathrm{E}+01$ & $6.22 \mathrm{E}+00$ & $\mathrm{cpt}$ \\
\hline 111.4 & $1.93 \mathrm{E}+01$ & $5.37 \mathrm{E}+00$ & $\mathrm{cpt}$ \\
\hline 110.4 & $1.52 \mathrm{E}+01$ & $4.23 \mathrm{E}+00$ & $\mathrm{cpt}$ \\
\hline 109.4 & $1.11 \mathrm{E}+01$ & $3.10 \mathrm{E}+00$ & $\mathrm{cpt}$ \\
\hline 108.4 & $1.41 \mathrm{E}+01$ & $3.90 \mathrm{E}+00$ & $\mathrm{cpt}$ \\
\hline 107.4 & $1.49 \mathrm{E}+01$ & $4.14 \mathrm{E}+00$ & $\mathrm{cpt}$ \\
\hline 106.4 & $1.69 \mathrm{E}+01$ & $4.69 \mathrm{E}+00$ & $\mathrm{cpt}$ \\
\hline 105.4 & $1.77 \mathrm{E}+01$ & $4.91 \mathrm{E}+00$ & $\mathrm{cpt}$ \\
\hline 104.4 & $2.18 \mathrm{E}+01$ & $6.05 \mathrm{E}+00$ & $\mathrm{cpt}$ \\
\hline 103.4 & $1.91 \mathrm{E}+01$ & $5.31 \mathrm{E}+00$ & $\mathrm{cpt}$ \\
\hline 102.4 & $1.49 \mathrm{E}+01$ & $4.13 \mathrm{E}+00$ & $\mathrm{cpt}$ \\
\hline 101.4 & $1.27 \mathrm{E}+01$ & $3.53 \mathrm{E}+00$ & $\mathrm{cpt}$ \\
\hline 100.4 & $1.41 \mathrm{E}+01$ & $3.91 \mathrm{E}+00$ & $\mathrm{cpt}$ \\
\hline 99.4 & $1.00 \mathrm{E}+02$ & $2.78 \mathrm{E}+01$ & $\mathrm{cpt}$ \\
\hline 98.4 & $5.86 \mathrm{E}-02$ & $1.63 \mathrm{E}-02$ & $\mathrm{cpt}$ \\
\hline
\end{tabular}




\section{Conductivity Data at RPT9}

\begin{tabular}{|c|c|c|c|}
\hline $\begin{array}{c}\text { Elevation } \\
\text { (ft) }\end{array}$ & $\begin{array}{c}\text { Kh } \\
\text { (ft/day) }\end{array}$ & $\begin{array}{c}\text { Kv } \\
\text { (ft/day) }\end{array}$ & $\begin{array}{c}\text { Data } \\
\text { Source }\end{array}$ \\
\hline 301.2 & $3.72 E+00$ & $1.03 E+00$ & $\mathrm{cpt}$ \\
\hline 300.2 & $3.85 E+00$ & $1.07 \mathrm{E}+00$ & $\mathrm{cpt}$ \\
\hline 299.2 & $4.10 E+00$ & $1.14 \mathrm{E}+00$ & cpt \\
\hline 298.2 & $5.06 \mathrm{E}+00$ & $1.40 \mathrm{E}+00$ & cpt \\
\hline 297.2 & $5.27 \mathrm{E}+00$ & $1.46 \mathrm{E}+00$ & cpt \\
\hline 296.2 & $6.22 \mathrm{E}+00$ & $1.73 \mathrm{E}+00$ & cpt \\
\hline 295.2 & $2.47 E+00$ & $6.85 \mathrm{E}-01$ & $\mathrm{cpt}$ \\
\hline 294.2 & $3.40 \mathrm{E}-04$ & $1.00 \mathrm{E}-04$ & cpt \\
\hline 293.2 & $3.60 \mathrm{E}-04$ & $1.00 \mathrm{E}-04$ & cpt \\
\hline 292.2 & $3.50 \mathrm{E}-04$ & $1.00 \mathrm{E}-04$ & cpt \\
\hline 291.2 & $3.30 \mathrm{E}-04$ & $9.00 E-05$ & $\mathrm{cpt}$ \\
\hline 290.2 & $3.30 \mathrm{E}-04$ & $9.00 \mathrm{E}-05$ & cpt \\
\hline 289.2 & $3.40 \mathrm{E}-04$ & $9.00 \mathrm{E}-05$ & cpt \\
\hline 288.2 & $3.30 E-04$ & $9.00 \mathrm{E}-05$ & $\mathrm{cpt}$ \\
\hline 287.2 & $3.40 \mathrm{E}-04$ & $9.00 \mathrm{E}-05$ & cpt \\
\hline 286.2 & $3.50 \mathrm{E}-04$ & $1.00 \mathrm{E}-04$ & cpt \\
\hline 285.2 & $2.88 E-01$ & $8.01 \mathrm{E}-02$ & cpt \\
\hline 284.2 & $3.74 \mathrm{E}+00$ & $1.04 E+00$ & cpt \\
\hline 283.2 & $2.85 E-01$ & $7.91 \mathrm{E}-02$ & $\mathrm{cpt}$ \\
\hline 282.2 & $1.07 \mathrm{E}+01$ & $2.98 \mathrm{E}+00$ & cpt \\
\hline 281.2 & $9.38 E+00$ & $2.61 \mathrm{E}+00$ & cpt \\
\hline 280.2 & $1.17 \mathrm{E}+01$ & $3.26 \mathrm{E}+00$ & cpt \\
\hline 279.2 & $3.40 \mathrm{E}-04$ & $9.00 \mathrm{E}-05$ & cpt \\
\hline 278.2 & $1.59 \mathrm{E}+01$ & $4.42 \mathrm{E}+00$ & $\mathrm{cpt}$ \\
\hline 277.2 & $2.03 E+01$ & $5.62 \mathrm{E}+00$ & $\overline{c p t}$ \\
\hline 276.2 & $5.70 \mathrm{E}+01$ & $1.58 \mathrm{E}+01$ & cpt \\
\hline 275.2 & $4.50 \mathrm{E}+01$ & $1.25 \mathrm{E}+01$ & cpt \\
\hline 274.2 & $3.05 \mathrm{E}+01$ & $8.47 \mathrm{E}+00$ & cpt \\
\hline 273.2 & $9.26 \mathrm{E}+00$ & $2.57 \mathrm{E}+00$ & cpt \\
\hline 272.2 & $8.38 \mathrm{E}-01$ & $2.33 \mathrm{E}-01$ & cpt \\
\hline 271.2 & $5.23 E-02$ & $1.45 \mathrm{E}-02$ & $\mathrm{cpt}$ \\
\hline 270.2 & $8.90 \mathrm{E}-04$ & $2.50 E-04$ & $\mathrm{cpt}$ \\
\hline 269.2 & $1.03 E-01$ & $2.87 E-02$ & $\mathrm{cpt}$ \\
\hline 268.2 & $2.52 \mathrm{E}+01$ & $6.99 \mathrm{E}+00$ & $\mathrm{cpt}$ \\
\hline 267.2 & $5.98 \mathrm{E}-01$ & $1.66 \mathrm{E}-01$ & $\mathrm{cpt}$ \\
\hline 266.2 & $6.29 \mathrm{E}-01$ & $1.75 \mathrm{E}-01$ & $\mathrm{cpt}$ \\
\hline 265.2 & $3.76 \mathrm{E}-03$ & $1.04 \mathrm{E}-03$ & cpt \\
\hline 264.2 & $3.35 \mathrm{E}-03$ & $9.30 \mathrm{E}-04$ & $\mathrm{cpt}$ \\
\hline 263.2 & $6.60 \mathrm{E}-04$ & $1.80 \mathrm{E}-04$ & cpt \\
\hline 262.2 & $6.10 \mathrm{E}-04$ & $1.70 \mathrm{E}-04$ & $\mathrm{cpt}$ \\
\hline 261.2 & $7.10 \mathrm{E}-04$ & $2.00 \mathrm{E}-04$ & cpt \\
\hline 260.2 & $4.49 \mathrm{E}-02$ & $1.25 \mathrm{E}-02$ & $\mathrm{cpt}$ \\
\hline 259.2 & $2.80 \mathrm{E}-01$ & $7.77 \mathrm{E}-02$ & $\mathrm{cpt}$ \\
\hline 258.2 & $1.95 \mathrm{E}-01$ & $5.41 E-02$ & cpt \\
\hline 257.2 & $8.52 E-03$ & $2.37 \mathrm{E}-03$ & $\mathrm{cpt}$ \\
\hline 256.2 & $1.30 \mathrm{E}-03$ & $3.60 \mathrm{E}-04$ & $\mathrm{cpt}$ \\
\hline 255.2 & $9.40 \mathrm{E}-03$ & $2.61 \mathrm{E}-03$ & $\mathrm{cpt}$ \\
\hline 254.2 & $5.79 \mathrm{E}-03$ & 1.61E-03 & cpt \\
\hline
\end{tabular}


Conductivity Data at RPT9

\begin{tabular}{|c|c|c|c|}
\hline $\begin{array}{c}\text { Elevation } \\
\text { (ft) }\end{array}$ & $\begin{array}{c}\text { Kh } \\
\text { (ft/day) }\end{array}$ & $\begin{array}{c}\text { Kv } \\
\text { (ft/day) }\end{array}$ & $\begin{array}{c}\text { Data } \\
\text { Source }\end{array}$ \\
\hline 253.2 & $4.03 E+00$ & $1.12 E+00$ & $\mathrm{cpt}$ \\
\hline 252.2 & $6.55 E-02$ & $1.82 \mathrm{E}-02$ & $\mathrm{cpt}$ \\
\hline 251.2 & $6.10 \mathrm{E}-04$ & 1.70E-04 & $\mathrm{cpt}$ \\
\hline 250.2 & $3.58 \mathrm{E}+00$ & $9.95 \mathrm{E}-01$ & $\mathrm{cpt}$ \\
\hline 249.2 & $2.85 E+01$ & $7.91 E+00$ & $\mathrm{cpt}$ \\
\hline 248.2 & $7.30 \mathrm{E}-04$ & $2.00 \mathrm{E}-04$ & $\mathrm{cpt}$ \\
\hline 247.2 & $5.90 \mathrm{E}-04$ & $1.60 \mathrm{E}-04$ & $\overline{c p t}$ \\
\hline 246.2 & $5.80 \mathrm{E}-04$ & $1.60 \mathrm{E}-04$ & cpt \\
\hline 245.2 & $8.83 E-02$ & $2.45 \mathrm{E}-02$ & cpt \\
\hline 244.2 & 3.11E-02 & $8.63 E-03$ & $\mathrm{cpt}$ \\
\hline 243.2 & $9.00 E+00$ & $2.50 E+00$ & $\mathrm{cpt}$ \\
\hline 242.2 & $6.82 \mathrm{E}+00$ & $1.89 \mathrm{E}+00$ & cpt \\
\hline 241.2 & $6.41 E+00$ & $1.78 E+00$ & cpt \\
\hline 240.2 & $7.89 \mathrm{E}+00$ & $2.19 \mathrm{E}+00$ & cpt \\
\hline 239.2 & $1.09 E+01$ & $3.01 E+00$ & cpt \\
\hline 238.2 & $7.91 \mathrm{E}+00$ & $2.20 E+00$ & $\mathrm{cpt}$ \\
\hline 237.2 & $2.44 \mathrm{E}-02$ & $6.79 \mathrm{E}-03$ & $\mathrm{cpt}$ \\
\hline 236.2 & $7.59 \mathrm{E}+00$ & $2.11 \mathrm{E}+00$ & $\mathrm{cpt}$ \\
\hline 235.2 & $1.03 E+01$ & $2.86 E+00$ & $\mathrm{cpt}$ \\
\hline 234.2 & $9.24 E+00$ & $2.57 E+00$ & cpt \\
\hline 233.2 & $2.87 E+01$ & $7.98 \mathrm{E}+00$ & $\mathrm{cpt}$ \\
\hline 232.2 & $1.30 \mathrm{E}+01$ & $3.60 E+00$ & $\mathrm{cpt}$ \\
\hline 231.2 & $1.70 E+01$ & $4.72 E+00$ & $\mathrm{cpt}$ \\
\hline 230.2 & $2.15 \mathrm{E}+01$ & $5.97 E+00$ & cpt \\
\hline 229.2 & $1.64 E+01$ & $4.55 E+00$ & $\mathrm{cpt}$ \\
\hline 228.2 & $3.66 \mathrm{E}-01$ & $1.02 \mathrm{E}-01$ & cpt \\
\hline 227.2 & $5.96 \mathrm{E}-01$ & $1.66 \mathrm{E}-01$ & $\overline{c p t}$ \\
\hline 226.2 & $1.06 E+01$ & $2.94 E+00$ & $\mathrm{cpt}$ \\
\hline 225.2 & $1.39 E+01$ & $3.85 E+00$ & cpt \\
\hline 224.2 & $9.16 \mathrm{E}+00$ & $2.54 \mathrm{E}+00$ & $\mathrm{cpt}$ \\
\hline 223.2 & $2.46 E-03$ & $6.80 \mathrm{E}-04$ & cpt \\
\hline 222.2 & $3.85 \mathrm{E}-01$ & $1.07 \mathrm{E}-01$ & cpt \\
\hline 221.2 & $1.50 \mathrm{E}+00$ & 4.17E-01 & cpt \\
\hline 220.2 & $1.35 \mathrm{E}+00$ & $3.74 E-01$ & cpt \\
\hline 219.2 & $1.64 \mathrm{E}+01$ & $4.54 E+00$ & cpt \\
\hline 218.2 & $1.44 E+01$ & $4.01 E+00$ & cpt \\
\hline 217.2 & $1.07 E+01$ & $2.97 E+00$ & cpt \\
\hline 216.2 & $1.00 E+01$ & $2.78 E+00$ & $\mathrm{cpt}$ \\
\hline 215.2 & $8.11 E+00$ & $2.25 E+00$ & $c p t$ \\
\hline 214.2 & $3.82 E+00$ & $1.06 E+00$ & $c p t$ \\
\hline 213.2 & $6.12 E-01$ & 1.70E-01 & $\mathrm{cpt}$ \\
\hline 212.2 & $4.27 E+00$ & $1.19 E+00$ & cpt \\
\hline 211.2 & 2.03E-03 & $5.60 \mathrm{E}-04$ & cpt \\
\hline 210.2 & $2.30 E+01$ & $6.38 E+00$ & $\mathrm{cpt}$ \\
\hline 209.2 & $4.17 E+01$ & $1.16 \mathrm{E}+01$ & cpt \\
\hline 208.2 & $5.76 E+01$ & $1.60 \mathrm{E}+01$ & cpt \\
\hline 207.2 & $5.38 \mathrm{E}+01$ & $1.49 \mathrm{E}+01$ & cpt \\
\hline 206.2 & $4.36 \mathrm{E}+01$ & $1.21 \mathrm{E}+01$ & $\mathrm{cpt}$ \\
\hline
\end{tabular}


Conductivity Data at RPT9

\begin{tabular}{|c|c|c|c|}
\hline $\begin{array}{c}\text { Elevation } \\
\text { (ft) }\end{array}$ & $\begin{array}{c}\text { Kh } \\
\text { (ft/day) }\end{array}$ & $\begin{array}{c}\text { Kv } \\
\text { (ft/day) }\end{array}$ & $\begin{array}{c}\text { Data } \\
\text { Source }\end{array}$ \\
\hline 205.2 & $3.32 E+01$ & $9.21 E+00$ & cpt \\
\hline 204.2 & $2.72 E+01$ & $7.56 \mathrm{E}+00$ & cpt \\
\hline 203.2 & $3.25 \mathrm{E}+01$ & $9.04 \mathrm{E}+00$ & $\mathrm{cpt}$ \\
\hline 202.2 & $3.38 E+01$ & $9.38 E+00$ & $\mathrm{cpt}$ \\
\hline 201.2 & $2.97 E+01$ & $8.25 E+00$ & $\mathrm{cpt}$ \\
\hline 200.2 & $1.36 E+00$ & $3.77 E-01$ & $\mathrm{cpt}$ \\
\hline 199.2 & $2.42 \mathrm{E}-03$ & $6.70 \mathrm{E}-04$ & cpt \\
\hline 198.2 & $2.19 \mathrm{E}-01$ & $6.08 \mathrm{E}-02$ & cpt \\
\hline 197.2 & $7.35 E-03$ & $2.04 E-03$ & cpt \\
\hline 196.2 & $1.94 E+01$ & $5.38 E+00$ & cpt \\
\hline 195.2 & $4.49 E+00$ & $1.25 E+00$ & cpt \\
\hline 194.2 & $1.00 E+02$ & $2.78 E+01$ & cpt \\
\hline 193.2 & $1.00 \mathrm{E}+02$ & $2.78 E+01$ & cpt \\
\hline 192.2 & $1.00 E+02$ & $2.78 E+01$ & $\mathrm{cpt}$ \\
\hline 191.2 & $1.00 \mathrm{E}+02$ & $2.78 E+01$ & cpt \\
\hline 190.2 & $1.00 E+02$ & $2.78 E+01$ & cpt \\
\hline 189.2 & $1.00 E+02$ & $2.78 E+01$ & cpt \\
\hline 188.2 & $5.58 E+01$ & $1.55 E+01$ & $\mathrm{cpt}$ \\
\hline 187.2 & $3.89 E+01$ & $1.08 E+01$ & $\mathrm{cpt}$ \\
\hline 186.2 & $3.37 E+01$ & $9.35 E+00$ & $\mathrm{cpt}$ \\
\hline 185.2 & $4.03 E+01$ & $1.12 E+01$ & cpt \\
\hline 184.2 & $3.92 \mathrm{E}+01$ & $1.09 E+01$ & $\mathrm{cpt}$ \\
\hline 183.2 & $2.57 E+01$ & $7.13 E+00$ & cpt \\
\hline 182.2 & $1.55 E+00$ & $4.32 E-01$ & cpt \\
\hline 181.2 & $5.50 E-02$ & $1.53 \mathrm{E}-02$ & cpt \\
\hline 180.2 & $1.16 \mathrm{E}-03$ & $3.20 \mathrm{E}-04$ & cpt \\
\hline 179.2 & $9.80 E-04$ & $2.70 E-04$ & $\mathrm{cpt}$ \\
\hline 178.2 & $4.47 E-02$ & $1.24 \mathrm{E}-02$ & cpt \\
\hline 177.2 & $5.12 \mathrm{E}+01$ & $1.42 E+01$ & cpt \\
\hline 176.2 & $1.00 E+02$ & $2.78 E+01$ & cpt \\
\hline 175.2 & $1.00 E+02$ & $2.78 E+01$ & cpt \\
\hline 174.2 & $1.00 E+02$ & $2.78 E+01$ & cpt \\
\hline 173.2 & $1.36 E+01$ & $3.78 E+00$ & $\mathrm{cpt}$ \\
\hline 172.2 & 2.19E-01 & $6.09 \mathrm{E}-02$ & cpt \\
\hline 171.2 & $1.70 \mathrm{E}-03$ & $4.70 \mathrm{E}-04$ & $\mathrm{cpt}$ \\
\hline 170.2 & $1.24 E-02$ & $3.44 \mathrm{E}-03$ & cpt \\
\hline 169.2 & $9.63 E-03$ & $2.68 \mathrm{E}-03$ & cpt \\
\hline 168.2 & $1.55 E-02$ & $4.31 E-03$ & $\mathrm{cpt}$ \\
\hline 167.2 & 1.12E-01 & $3.11 \mathrm{E}-02$ & cpt \\
\hline 166.2 & $6.63 E+00$ & $1.84 E+00$ & cpt \\
\hline 165.2 & $5.60 E+01$ & $1.56 E+01$ & cpt \\
\hline 164.2 & $7.71 \mathrm{E}+01$ & $2.14 E+01$ & cpt \\
\hline 163.2 & $8.51 E+01$ & $2.36 E+01$ & $\mathrm{cpt}$ \\
\hline 162.2 & $3.55 E-01$ & $9.87 \mathrm{E}-02$ & $\mathrm{cpt}$ \\
\hline 161.2 & $7.64 E-03$ & $2.12 \mathrm{E}-03$ & cpt \\
\hline 160.2 & $2.93 E+01$ & $8.13 E+00$ & $\mathrm{cpt}$ \\
\hline 159.2 & $3.96 E-03$ & $1.10 \mathrm{E}-03$ & cpt \\
\hline 158.2 & $1.75 E+00$ & $4.85 \mathrm{E}-01$ & cpt \\
\hline
\end{tabular}


Conductivity Data at RPT9

\begin{tabular}{|c|c|c|c|}
\hline $\begin{array}{c}\text { Elevation } \\
\text { (ft) }\end{array}$ & $\begin{array}{c}\mathrm{Kh} \\
\text { (ft/day) }\end{array}$ & $\begin{array}{c}\text { Kv } \\
\text { (ft/day) }\end{array}$ & $\begin{array}{l}\text { Data } \\
\text { Source }\end{array}$ \\
\hline 157.2 & 2.03E-02 & $5.64 E-03$ & $\mathrm{cpt}$ \\
\hline 156.2 & $4.52 E-03$ & $1.26 E-03$ & $\mathrm{cpt}$ \\
\hline 155.2 & $1.76 \mathrm{E}+01$ & $4.89 E+00$ & $\mathrm{cpt}$ \\
\hline 154.2 & $3.59 E+01$ & $9.96 \mathrm{E}+00$ & $\mathrm{cpt}$ \\
\hline 153.2 & $3.02 E+01$ & $8.39 E+00$ & $\mathrm{cpt}$ \\
\hline 152.2 & $2.24 E+01$ & $6.22 E+00$ & $\mathrm{cpt}$ \\
\hline 151.2 & $2.50 E+01$ & $6.94 E+00$ & $\mathrm{cpt}$ \\
\hline 150.2 & $2.92 E+01$ & $8.11 E+00$ & $\mathrm{cpt}$ \\
\hline 149.2 & $2.56 E+01$ & $7.12 E+00$ & $c p t$ \\
\hline 148.2 & $4.83 E+01$ & $1.34 E+01$ & $\mathrm{cpt}$ \\
\hline 147.2 & $4.47 E+01$ & $1.24 \mathrm{E}+01$ & cpt \\
\hline 146.2 & $4.90 \mathrm{E}+01$ & $1.36 E+01$ & $\mathrm{cpt}$ \\
\hline 145.2 & $9.69 E+01$ & $2.69 \mathrm{E}+01$ & cpt \\
\hline 144.2 & $8.58 E+01$ & $2.38 E+01$ & $\mathrm{cpt}$ \\
\hline 143.2 & $7.57 E+01$ & $2.10 E+01$ & cpt \\
\hline 142.2 & $6.71 E+01$ & $1.86 \mathrm{E}+01$ & cpt \\
\hline 141.2 & $5.08 E+01$ & $1.41 E+01$ & cpt \\
\hline 140.2 & $4.21 E+01$ & $1.17 E+01$ & cpt \\
\hline 139.2 & $5.29 E+01$ & $1.47 E+01$ & cpt \\
\hline 138.2 & $3.14 \mathrm{E}+01$ & $8.73 E+00$ & $\mathrm{cpt}$ \\
\hline 137.2 & $8.12 E+01$ & $2.26 E+01$ & cpt \\
\hline 136.2 & $8.66 E+01$ & $2.41 E+01$ & $\mathrm{cpt}$ \\
\hline 135.2 & $9.04 \mathrm{E}+01$ & $2.51 E+01$ & cpt \\
\hline 134.2 & $1.00 \mathrm{E}+02$ & $2.78 E+01$ & $\mathrm{cpt}$ \\
\hline 133.2 & $5.99 \mathrm{E}+01$ & $1.66 \mathrm{E}+01$ & cpt \\
\hline 132.2 & $3.44 E+01$ & $9.54 \mathrm{E}+00$ & $\mathrm{cpt}$ \\
\hline 131.2 & $2.00 \mathrm{E}+01$ & $5.56 \mathrm{E}+00$ & cpt \\
\hline 130.2 & $2.74 \mathrm{E}+01$ & $7.61 \mathrm{E}+00$ & cpt \\
\hline 129.2 & $1.42 \mathrm{E}+01$ & $3.95 E+00$ & $\mathrm{cpt}$ \\
\hline 128.2 & $2.15 E+01$ & $5.97 E+00$ & $\mathrm{cpt}$ \\
\hline 127.2 & $2.52 E+01$ & $6.99 \mathrm{E}+00$ & cpt \\
\hline 126.2 & $1.82 \mathrm{E}+01$ & $5.06 \mathrm{E}+00$ & $\mathrm{cpt}$ \\
\hline 125.2 & $1.87 E+01$ & $5.18 \mathrm{E}+00$ & $\mathrm{cpt}$ \\
\hline 124.2 & $2.48 E+01$ & $6.89 E+00$ & $\mathrm{cpt}$ \\
\hline 123.2 & $1.41 \mathrm{E}+01$ & $3.92 E+00$ & $\mathrm{cpt}$ \\
\hline 122.2 & $1.86 E+01$ & $5.16 E+00$ & $\mathrm{cpt}$ \\
\hline 121.2 & $1.79 E+01$ & $4.98 \mathrm{E}+00$ & $\mathrm{cpt}$ \\
\hline 120.2 & $1.68 E+01$ & $4.68 \mathrm{E}+00$ & $\mathrm{cpt}$ \\
\hline 119.2 & $1.84 \mathrm{E}+01$ & $5.11 E+00$ & cpt \\
\hline 118.2 & $1.61 E+01$ & $4.47 E+00$ & $\mathrm{cpt}$ \\
\hline 117.2 & $2.07 E+01$ & $5.74 \mathrm{E}+00$ & $\mathrm{cpt}$ \\
\hline 116.2 & $1.74 \mathrm{E}+01$ & $4.83 E+00$ & $\mathrm{cpt}$ \\
\hline 115.2 & $1.67 \mathrm{E}+01$ & $4.65 E+00$ & cpt \\
\hline 114.2 & $1.75 E+01$ & $4.86 E+00$ & cpt \\
\hline 113.2 & $1.60 \mathrm{E}+01$ & $4.45 E+00$ & cpt \\
\hline 112.2 & $1.54 E+01$ & $4.29 E+00$ & cpt \\
\hline 111.2 & $1.43 E+01$ & $3.97 E+00$ & cpt \\
\hline 110.2 & $1.91 \mathrm{E}+00$ & $5.30 \mathrm{E}-01$ & $\mathrm{cpt}$ \\
\hline
\end{tabular}


Conductivity Data at RPT9

\begin{tabular}{|c|c|c|c|}
\hline $\begin{array}{c}\text { Elevation } \\
\text { (ft) }\end{array}$ & $\begin{array}{c}\text { Kh } \\
\text { (ft/day) }\end{array}$ & $\begin{array}{c}\text { Kv } \\
\text { (ft/day) }\end{array}$ & $\begin{array}{c}\text { Data } \\
\text { Source }\end{array}$ \\
\hline 109.2 & $1.07 \mathrm{E}+01$ & $2.98 \mathrm{E}+00$ & $\mathrm{cpt}$ \\
\hline 108.2 & $1.15 \mathrm{E}+01$ & $3.19 \mathrm{E}+00$ & $\mathrm{cpt}$ \\
\hline 107.2 & $1.35 \mathrm{E}+01$ & $3.74 \mathrm{E}+00$ & $\mathrm{cpt}$ \\
\hline 106.2 & $1.32 \mathrm{E}+01$ & $3.67 \mathrm{E}+00$ & $\mathrm{cpt}$ \\
\hline 105.2 & $1.22 \mathrm{E}+01$ & $3.39 \mathrm{E}+00$ & $\mathrm{cpt}$ \\
\hline 104.2 & $1.17 \mathrm{E}+01$ & $3.25 \mathrm{E}+00$ & $\mathrm{cpt}$ \\
\hline 103.2 & $3.18 \mathrm{E}+01$ & $8.84 \mathrm{E}+00$ & $\mathrm{cpt}$ \\
\hline 102.2 & $4.14 \mathrm{E}-03$ & $1.15 \mathrm{E}-03$ & $\mathrm{cpt}$ \\
\hline 101.2 & $5.78 \mathrm{E}-03$ & $1.60 \mathrm{E}-03$ & $\mathrm{cpt}$ \\
\hline 100.2 & $1.85 \mathrm{E}-01$ & $5.14 \mathrm{E}-02$ & $\mathrm{cpt}$ \\
\hline 99.2 & $1.00 \mathrm{E}+02$ & $2.78 \mathrm{E}+01$ & $\mathrm{cpt}$ \\
\hline 98.2 & $5.07 \mathrm{E}+01$ & $1.41 \mathrm{E}+01$ & $\mathrm{cpt}$ \\
\hline
\end{tabular}




\section{Appendix G \\ Model Calibration Runs}


R-Reactor Area Run Sce

\begin{tabular}{|c|c|c|c|c|c|c|c|c|c|}
\hline Model & Objectives & Grid & & & RMS & & & & Averas \\
\hline Scenario & & Size & Gordon & lower & 'AA' & $A^{\prime}$ & Surface S. & Gordon & lower \\
\hline Run 1 & & 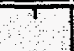 & ND & ND & ND & ND & ND & ND & ND \\
\hline Status & & 2 & ND & ND & ND & ND & ND & ND: & ND \\
\hline Run2 & & 2 & 0.5 & 26 & 28.3 & 125 & 74 & 0.5 & 2.6 \\
\hline Run 2A & Sensitivity & 2 & ND & ND & ND & ND & ND & ND & ND \\
\hline Run2B & Sensitivity & 2 & ND & ND & ND & ND & ND & ND & ND \\
\hline Run $2 \mathrm{C}$ & green clay sensitivity & 2 & ND & ND & ND & ND & ND & ND & ND \\
\hline Run 2D & Sensitivity & 2 & 1.3 & 1.2 & 30.6 & 12.4 & 13.5 & ND & $\overline{\mathrm{ND}}$ \\
\hline Run 3 & $\begin{array}{l}\text { Smooth conductivity } \\
\text { field }\end{array}$ & 2 & 12 & 0.6 & 30.0 & 13.5 & 13.2 & 1.2 & 0.6 \\
\hline Run $3 A$ & $\begin{array}{l}\text { Increase head } \\
\text { in 'AA' horizon }\end{array}$ & 2 & 1.3 & 4.2 & 26.0 & 13.9 & 8.8 & 1.3 & 4.2 \\
\hline Run 3B & $\begin{array}{l}\text { Increase head } \\
\text { in 'AA' horizon }\end{array}$ & 2 & 1.4 & 5.1 & 26.0 & 20.7 & ND & 1.4 & 5.1 \\
\hline Run 3C & $\begin{array}{l}\text { Increase head } \\
\text { in 'A' horizon }\end{array}$ & 2 & 1.2 & 1.3 & 27.0 & 12.8 & 3.9 & 1.2 & 1.3 \\
\hline Run 4 & $\begin{array}{l}\text { Increase head in } A^{\prime} \\
\text { and Surface Solis }\end{array}$ & 2 & 1.1 & 1.8 & 27.0 & 17.3 & 23.6 & 1.1 & -1.8 \\
\hline Run 4A & $\begin{array}{l}\text { Increase head } \\
\text { in 'AA' horizon }\end{array}$ & 2 & 1.3 & 2.6 & 19.0 & 14.2 & 19.0 & 1.3 & 2.6 \\
\hline Run 4B & $\begin{array}{l}\text { Increase head } \\
\text { in 'AA' horizon }\end{array}$ & 2 & 1.3 & 4.2 & 16.6 & 9.1 & 7.5 & 1.3 & 4.2 \\
\hline Run 4C & $\begin{array}{l}\text { Increase head } \\
\text { in 'AA' horizon }\end{array}$ & 2 & 1.3 & 5.3 & 14.0 & 12.6 & 15.0 & 1.3 & 5.3 \\
\hline Run 4D & $\begin{array}{l}\text { Increase head in 'A', } \\
\text { 'AA' and Surface } S \text {. }\end{array}$ & 2 & 1.4 & 7.2 & 10.0 & 7.9 & 8.8 & 1.4 & 7.2 \\
\hline Run 5 & $\begin{array}{c}\text { Smooth conductivity } \\
\text { field }\end{array}$ & 2 & 1.4 & 6.0 & 8.0 & 5.5 & 5.3 & 1.4 & 6.0 \\
\hline Run 5A & $\begin{array}{l}\text { Increase head } \\
\text { in 'A' horizon }\end{array}$ & 2 & 1.2 & 1.5 & 4.0 & 7.7 & 5.1 & 1.2 & 1.5 \\
\hline Run 5B & $\begin{array}{l}\text { Decrease head in } \\
\text { 'A' and Surface } S \text {. }\end{array}$ & 2 & 1.2 & 1.0 & 3.5 & 6.0 & 3.3 & 1.2 & 1.0 \\
\hline Run 5C & $\begin{array}{l}\text { Decrease head in } \\
\text { 'A' and Surface } S \text {. }\end{array}$ & 2 & 1.2 & 0.4 & 4.2 & 4.8 & 4.2 & 1.2 & 0.4 \\
\hline Run 5D & Sensitivity & 2 & 1.3 & 5.4 & 11.5 & 13.7 & 19.0 & 1.3 & 5.4 \\
\hline
\end{tabular}




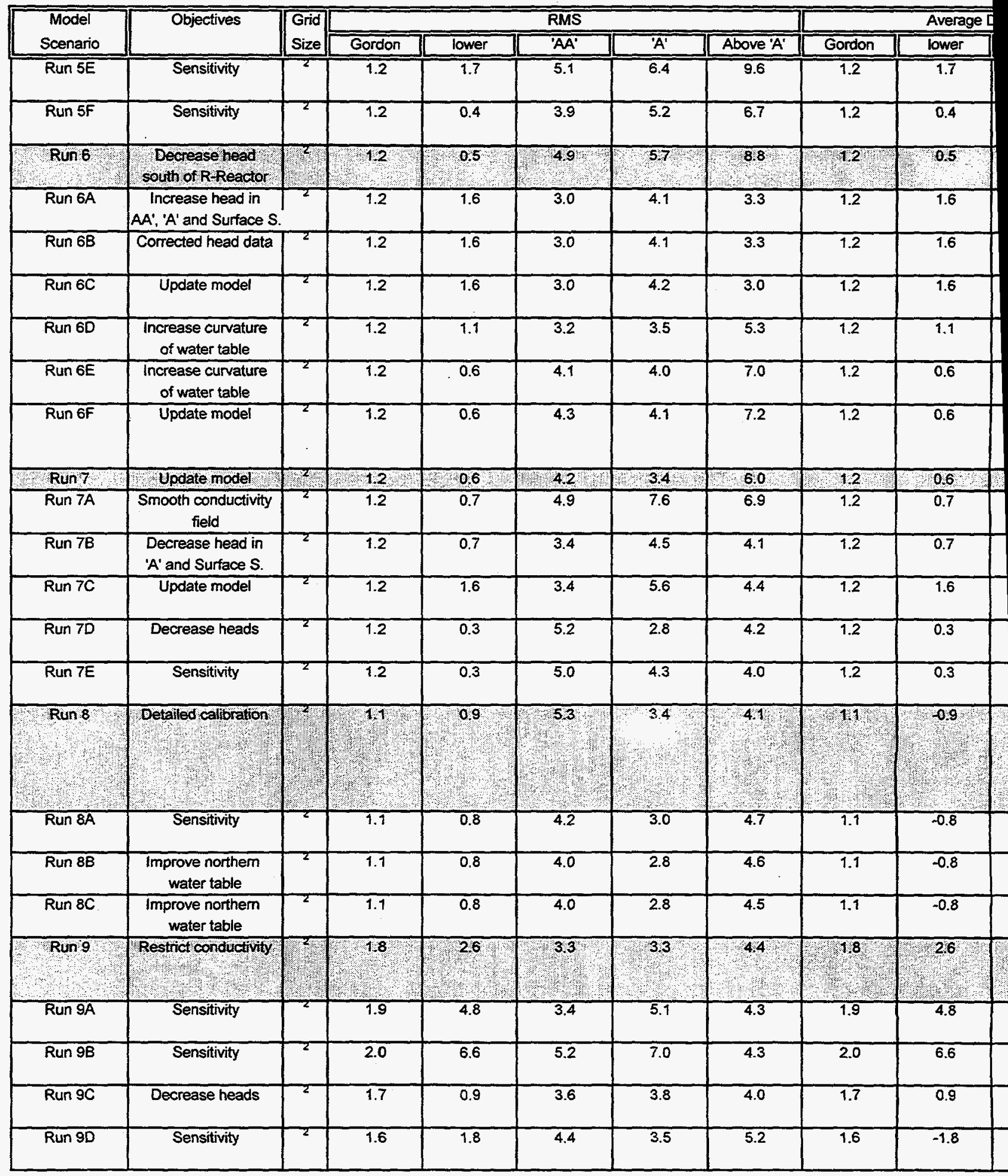

\section{ND - Not Determined}

- Number of columns $=92$, Number of Rows $=84$, Number of Layers $=9$

2 - Number of columns $=65$, Number of Rows $=62$, Number of Layers $=21$ 